TESIS DOCTORAL

\title{
CONTRIBUCIÓN AL ESTUDIO Y CARACTERIZACIÓN DE LA GENERACIÓN DE RUIDO DE FLUJO EN EL SISTEMA DE ESCAPE
}

\author{
presentada por \\ INMACULADA ANDRÉS VERDÚ
}

en el

DEPARTAMENTO DE MÁQUINAS Y MOTORES TÉRMICOS de la UNIVERSIDAD POLITÉCNICA DE VALENCIA

Para la obtención del grado de

DOCTORA EN CIENCIAS FÍSICAS

Valencia, Febrero de 2003 



\title{
CONTRIBUCIÓN AL ESTUDIO Y CARACTERIZACIÓN DE LA GENERACIÓN DE RUIDO DE FLUJO EN EL SISTEMA DE ESCAPE
}

\author{
Realizada por: \\ $\mathrm{D}^{\mathrm{a}}$. Inmaculada Andrés Verdú \\ Dirigida por: \\ Dr. D. Antonio Torregrosa Huguet
}

\section{TRIBUNAL CALIFICADOR:}

Presidente:

Secretario:

Vocales:

Vocales suplentes:
Dr. D. José María Desantes Fernández

Dr. D. Alberto Broatch Jacobi

Dr. D. Jaime LLinares Galiana

Dr. D. José Juan Salvá Monfort

Dra. Da ${ }^{a}$. María Jesús Hernández Lucas

Dr. D. Francisco Javier Fuenmayor Fernández

Dr. D. Magin Lapuerta Amigo

Valencia, Febrero de 2003 



\section{RESUMEN}

Los estudios de emisiones sonoras por vehículos han adquirido gran relevancia en los últimos años por dos razones: (1) el incremento de la sensibilización social hacia los fenómenos de contaminación ambiental entre los que se encuentra la contaminación acústica, y (2) las mayores exigencias del mercado (vehículos de mayores prestaciones a la vez que más confortables: confort acústico).

Dentro de la complejidad de los fenómenos de generación acústica por el vehículo, en esta tesis doctoral nos centraremos en una de las contribuciones del motor: en el ruido generado durante el proceso de escape. Esta componente sonora es la resultante de dos fenómenos distintos: el ruido pulsante originado en el proceso de llenado y vaciado de los cilindros, y el ruido de flujo asociado a los fenómenos de turbulencia desarrollados por la existencia de un flujo medio continuo que atraviesa el sistema de escape. De estos dos fenómenos será el estudio del ruido de flujo y su predicción el núcleo de nuestro trabajo, con la motivación añadida de que así como el ruido pulsante ha sido ampliamente estudiado y existen métodos de predicción del mismo, todo lo referente al ruido de flujo es en gran medida desconocido. A esto, se debe añadir que a medida que se ha ido reduciendo la contribución del ruido pulsante, fuente principal, la contribución del ruido de flujo al ruido de escape es cada vez más importante, tanto por su interacción con la acústica del sistema (pudiendo amplificar la contribución de la fuente principal), como por la existencia de silenciadores que actúan como fuentes generadoras del mismo, de forma que a altos regímenes de giro se constituye como la fuente dominante.

Para el estudio de la contribución del ruido de flujo al ruido de escape la presente tesis doctoral se ha estructurado en seis capítulos bien diferenciados:

En un primer capítulo se realiza un inventario de las distintas contribuciones que pueden distinguirse en el ruido total emitido por un vehículo, justificando a partir del estado actual del conocimiento de esta materia la importancia del estudio del ruido de flujo, así como las bases de la metodología desarrollada para llevarlo a cabo.

En un segundo capítulo se presenta un método de diagnóstico experimental de la fluctuación del caudal en la boca de la cánula de escape y se analizan los diferentes mecanismos físicos involucrados en el proceso de emisión sonora mediante la introducción de un modelo de emisión realista. Se establece la posibilidad de realizar un desacoplamiento entre las componentes sonoras de 
ruido pulsante y de ruido de flujo, mostrando que esta última componente se genera por la superposición de una componente de flujo estacionaria sobre la componente pulsante.

Una vez justificada la posibilidad de realizar el estudio de la contribución del ruido de flujo generado durante el proceso de escape como el effecto de un flujo estacionario, en el capítulo 3 se presenta una técnica de análisis experimental, intensimetría en conductos, que permite estudiar el ruido de flujo generado por elementos aislados del sistema de escape (de utilidad en la industria) mediante un banco de flujo frío estacionario. Técnica que permite, además, separar y conocer las contribuciones al ruido final radiado de las fuentes internas y externas al silenciador.

Desarrolladas las técnicas de diagnóstico experimental que nos proporcionan datos fiables sobre el ruido de flujo generado en los sistemas de escape, se procede en el cuarto capítulo al análisis de las emisiones globales realizadas durante el proceso de emisión del ruido de flujo (valores a los que se refieren los límites establecidos en la legislación vigente, y por tanto responsables de que un sistema de escape pase o no la normativa).

Este análisis experimental se complementa con un estudio computacional (presentado en el capítulo 5). De esta forma se profundiza en el conocimiento de los principales mecanismos de generación sonora en el interior del silenciador, identificando las fuentes y obteniendo leyes de escalado que establezcan una relación directa entre el ruido de flujo emitido y los parámetros geométricos del silenciador. La finalidad de este estudio es la posibilidad de establecer criterios de diseño de silenciadores que cumplan con la legislación y con las expectativas de usuarios y fabricantes.

Finalmente en el capítulo 6, se realiza una síntesis de los resultados expuestos en los capítulos anteriores, estableciendo las bases de una metodología adecuada para el estudio y estimación del ruido de flujo generado durante el proceso de escape en el motor, que aborda diferentes aspectos de su generación y proporciona información relevante desde el punto de vista de la industria. 


\section{ABSTRACT}

The studies of the vehicle noise emissions has been acquired a great relevance over the last few years for two reasons: (1) the increased social sensitivity to environmental contamination phenomena among which acoustic contamination is found, and (2) to the highest market requirements (higher "capability" vehicles at the same time as more comfortable ones: acoustic comfort).

Within the complexity of the vehicle acoustic generation phenomena in this doctoral thesis, we will focus on one of the engine contributions: the exhaust noise. This component of noise is the result of two different phenomena: pulsating noise originated in the gas exchange process in the cylinders, and the flow noise induced by the turbulence phenomena developed by the mean flow that goes through the exhaust system. From these two phenomena the core of our study is flow noise and its prediction, under the motivation that as well as the pulsating noise being extensively studied and there being some prediction methods in it, the flow noise is unknown to a great extent. To this we should add, that while the pulsating noise contribution, main source, has been reduced the flow noise contribution has become more and more important in exhaust noise. The relevance of flow noise contribution is associated with its interaction with the acoustics in the system (it could amplify the main source contribution) and the existence of mufflers that work as flow noise generation sources.

For the flow noise contribution study, the present doctoral thesis has been structured in six well-defined chapters.

In the first chapter there is a summary of the different contributions that can be distinguished in the overall vehicle emitted noise. The importance of the flow noise study is justified from the present state of knowledge in this subject, and also the methodology bases used to perform it.

In the second chapter, an experimental procedure for the estimation of the volume velocity fluctuation at the open end of the exhaust tailpipe of an internal combustion engine is presented. The different physical mechanisms involved in the emission process are analysed through the introduction of a realistic emission model. The possibility to consider that the pulsating component of the noise and the flow noise are decoupled to a certain extent is established, which means that this last component is generated by a mean "steady" flow superimposed to a pulsating flow. 
Once the possibility of studying the contribution of flow noise generated during the exhaust process as a steady flow effect has been justified. In the third chapter an experimental technique, sound intensity from in-duct measurements, is presented to analyse it. This technique allows the study of the flow noise generation by the individual component of an exhaust system (useful in the automotive industry) on a cold flow test bench. The proposed technique also allows to separate and to know the contributions in the final radiated noise of the internal and external sources of the muffler.

Once the experimental procedures to obtain reliable flow data of flow noise generated in the exhaust systems has been developed. In the fourth chapter we proceed with the analysis of the overall noise emission carried out during the flow noise emission process (they are the values that valid legislation is referred to, for this reason, they are responsible for whether an exhaust system passes the regulations or not).

This experimental analysis is completed by means of a computational study (presented in the fifth chapter). In this way are fathomed in the knowledge of the main sound generation mechanism inside the muffler, it allow to identify the sound sources and to obtain the scaling laws that establish a direct relation between the emitted noise and the geometrical parameters of the muffler. The aim of this study is to found design criteria that reproduce the best compromise among: to comply the legislation and customer and manufacturer expectations.

Finally, in the sixth chapter, a synthesis of the exposed results in the previous chapters is carried out, establishing the bases of an appropriate methodology for the study and estimation of the flow noise generated in the exhaust engine process. Methodology that broaches different aspects of flow noise generation and gives important information to the automotive industry's point of view. 


\section{RESUM}

Els estudis del'emissió sonora pels vehicles ha anat adquirint gran rellevància en els últims anys per dos raons: (1) l'increment de la sensibilització social cap als fenòmens de contaminació ambiental entre els que es troba la contaminació acústica i (2) les majors exigències del mercat (vehicles de majors prestacions a la vegada que més confortables: confort acústic).

Dins de la complexitat dels fenòmens de generació acústica pel vehicle, en esta tesi doctoral ens centrarem en una de les contribucions del motor: el soroll generat durant el procés de escapament. Esta component sonora es el resultat de dos fenòmens distints: el soroll polsant originat en el procés de ompliment i buidatge dels cilindres, i el soroll de flux associat als fenòmens de turbulència desenvolupats per l'existència d'un flux mitjà continu que travessa el sistema de escapament. D'estos dos fenòmens serà l'estudi del soroll de flux i la seua predicció el nucli del nostre treball, amb la motivació afegida de que així com el soroll polsant ha sigut àmpliament estudiat i hi ha mètodes de predicció del mateix, el soroll de flux és en gran mesura desconegut. A açò, s'ha d'afegir que a mesura que s'ha anat reduint la contribució del soroll polsant, font principal, la contribució del soroll de flux al soroll de escapament és cada vegada més important, tant per la seua interacció amb l'acústica del sistema (podent amplificar la contribució de la font principal), com per l'existència de silenciadors que actuen com a fonts generadores del mateix, de forma que a alts règims de gir es constituix com la font dominant.

Per a l'estudi de la contribució del soroll de flux al soroll de escapament la present tesi doctoral s'ha estructurat en sis capítols ben diferenciats:

En un primer capítol es realitza un inventari de les distintes contribucions que poden distingir-se en el soroll total emés per un vehicle, justificant a partir de l'estat actual del coneixement de esta matèria, la importància de l'estudi del soroll de flux, així com les bases de la metodologia desenvolupada per a portar-ho a cap.

En un segon capítol es presenta un mètode de diagnòstic experimental de la fluctuació del cabal en la boca de la cànula de escapament i s'analitzen els diferents mecanismes físics involucrats en el procés d'emissió sonora per mitjà de l'introducció d'un model d'emissió realista. S'establix la possibilitat de realitzar un desacoblament entre les components sonores de soroll polsant i de soroll de flux, mostrant que esta última component es genera per la superposició d'una component de flux estacionária sobre la component polsant. 
Justificada la possibilitat de realitzar l'estudi de la contribució del soroll de flux generat durant el procés de escapament com l'efecte d'un flux estacionari, en el capítol 3 es presenta una tècnica d'anàlisi experimental, intensimetria en conductes, que permet estudiar el soroll de flux generat per elements aillats del sistema de escapament (de gran utilitat en la indústria) per mitjà de un banc de flux fred estacionari. Tècnica que permet, a més, separar i conéixer les contribucions al soroll final radiat de les fonts internes i externes al silenciador.

Desenvolupades les tècniques de diagnòstic experimental que ens proporcionen dades fiables sobre el soroll de flux generat en els sistemes de escapament, es procedix en el quart capítol a l'anàlisi de les emissions globals realitzades durant el procés d'emissió del soroll de flux (valors a què es referixen els límits establits en la legislació vigent, i per tant responsables de que un sistema de escapament passe o no la normativa).

Este anàlisis experimental es complementa amb un estudi computacional (presentat al capítol 5). D'esta manera s'aprofundix en el coneixement dels principals mecanismes de generació sonora en l'interior del silenciador, identificant les fonts i obtenint lleis d'escalat que establisquen una relació directa entre el soroll emés i els paràmetres geomètrics del silenciador. La finalitat d'este estudi és la possibilitat d'establir criteris de disseny que complisquen amb la legislació i amb les expectatives de usuaris i fabricants.

Finalment en el capítol 6, es realitza una síntesi dels resultats exposats en els capítols anteriors, establint les bases d'una metodologia adequada per a l'estudi i estimació del soroll de flux generat durant el procés de escapament en el motor, que aborda diferents aspectes de la seua generació i proporciona informació rellevant des del punt de vista de la indústria. 
A mi familia, por ser familia $y$ haberme regalado su cariño.

A Antón por haber creido en mi. 



\section{AGRADECIMIENTOS}

Quiero agradecer tantas cosas y a tanta gente que no se si las frases utilizadas y el espacio dedicado para ellos será suficiente. Por comenzar por algún sitio me gustaría agradecer en primer lugar a toda la línea de ruido el apoyo ofrecido durante todo este tiempo, en especial a mi director de Tesis Antonio Torregrosa por haberme ayudado a reecontrarme con el maravilloso mundo de la física y haberme enseñado que aunque en algunas ocasiones la tarea de la investigación resulte ardua, cualquier pequeño hallazgo recompensa todo el esfuerzo empleado. Del mismo modo, quiero agradecer a Alberto Broatch, la ayuda prestada en todo el desarrollo experimental de esta tesis doctoral. Me gustaría agradecer a los técnicos de esta línea, Adolfo Guzman (Fito), Miguel Cantó y Bernardo Planells la gran labor realizada no sólo porque sin su buen hacer esta tesis doctoral no podría haberse llevado a cabo, sino porque su paciencia y dedicación han conseguido acercar esta mente tan teórica al mundo de la experimentación. Por último, agradecer a mis compañeros de despacho David y José Manuel su infinita paciencia demostrada, porque soy consciente de que convivir con una doctoranda en fase terminal no es una tarea sencilla.

Agradecer a la línea 3D, especialmente a Xandra y a Antonio, sus consejos y ayuda, sin los cuales no me podría haber adentrado en el mundo de la simulación con el código FLuent.

Gracias a todos los amigos y compañeros, que han compartido conmigo la vida diaria en la CMT; gracias a Leo, Helena, Carmen, Vicente, Fran, JoseVi, Maria, Sergio, Hector, Xemary, Tania y un sin fin de gente por haber creado buen ambiente y haberme ayudado a disfrutar de la vida.

Quiero agradecer también al director del Departamento Francisco Payri el haberme brindado la posibilidad de realizar el doctorado en este grupo, en el que se puede seguir aprendiendo mientras se aplica la física a temas de actualidad.

Agradecer al grupo de administración Elvira, Carmina, Haby, Amparo y Elena toda la ayuda prestada, espero no haber sido demasiado pesada.

Finalmente, agradecer a todas las personas que han hecho posible que haya llegado hasta este punto, a mis padres por haberme permitido y animado a estudiar la mejor carrera del mundo que si bien no proporciona grandes recompensas económicas (como ya me decía mi padre) si te permite soñar despierta, a mis hermanos por estar siempre ahí, y a la gente que confió en mi y creyó que sería capaz de hacer una tesis doctoral en toda regla, gracias a Luisfe y a Antón. 



\section{Índice}

1 Introducción 1

1.1 Introducción . . . . . . . . . . . . . . . . . . . 3

1.2 Fuentes de ruido en el motor . . . . . . . . . . . . 3

1.3 Objetivos y desarrollo del trabajo . . . . . . . . . . . . . 10

2 Diagnóstico experimental del ruido de escape 15

2.1 Introducción . . . . . . . . . . . . . . . . . . . . . 17

2.2 Descripción del método . . . . . . . . . . . . . . . . . . 19

2.3 Estimación de la fluctuación de velocidad en la boca de la cánula de escape . . . . . . . . . . . . . . 20

2.3.1 Coeficiente de reflexión en el extremo abierto . . . . . . 24

2.3.2 Validación numérica . . . . . . . . . . . . . 27

2.3.3 Discusión sobre el error cometido en la estimación del caudal volumétrico . . . . . . . . . . . . . . . 38

2.4 Validación del método con líneas de escape reales: resultados y discusión . . . . . . . . . . . . . . . . . . 41

2.4.1 Análisis de la influencia del error del procedimiento propuesto en la estimación del ruido . . . . . . . . . . . 45

2.5 Evaluación del modelo de emisión . . . . . . . . . . . . . . . 47

2.6 Modelo monopolar . . . . . . . . . . . . . . . . . . . . . . . . 52

2.7 Modelo de emisión de un pistón vibrante . . . . . . . . . . . . 54

2.8 Evaluación de los modelos frente a las medidas experimentales 60

2.8.1 Medida de ruido . . . . . . . . . . . . . . . . 61

2.8.2 Resultados de la validación . . . . . . . . . . . . . 64 
2.9 Discusión de los resultados . . . . . . . . . . . . . . 70

2.10 Carácter estacionario del ruido de flujo en escapes . . . . . . 75

3 Estudio experimental del ruido de flujo estacionario $\quad 79$

3.1 Introducción . . . . . . . . . . . . . . . . . . . 81

3.2 Descripción del método de intensimetría en conductos . . . . . 82

3.2.1 Montaje experimental . . . . . . . . . . . . . 84

3.2.2 Método de cálculo de la intensidad radiada . . . . . . 88

3.3 Teoría de la descomposición de ondas . . . . . . . . . . . . 91

3.3.1 Método de los dos micrófonos . . . . . . . . . . . . . . 92

3.3.2 Descomposición de ondas mediante técnicas de conformación del haz. . . . . . . . . . . . . . . . . 94

3.3.3 Conformador del haz de mínima varianza con restricciones lineales $(\mathrm{LCMV}) \ldots \ldots$. . . . . . . . . 100

3.3.4 Consistencia del método de descomposición . . . . . . . 102

3.4 Validación de la potencia de la técnica de estimación de la intensidad . . . . . . . . . . . . . . . . 103

3.4.1 Análisis del proceso de emisión . . . . . . . . . . . . 108

4 Análisis del comportamiento de la fuente 115

4.1 Introducción . . . . . . . . . . . . . . . . . . . . . 117

4.2 Evolución histórica . . . . . . . . . . . . . . . . . 117

4.3 Bases teóricas sobre la generación y emisión de ruido de flujo . 119

4.3.1 Analogía de Lighthill . . . . . . . . . . . . . . . . 125

4.3.1.1 Descripción y justificación de la analogía de Lighthill . . . . . . . . . . . . . 125

4.3.1.2 Campo de esfuerzo externo equivalente . . . . 127

4.3.1.3 Mecanismos de conversión de energía . . . . . 132

4.3.1.4 Campos de radiación monopolar, dipolar y cuadripolar . . . . . . . . . . . . . 133

4.3.1.5 Análisis dimensional de la producción de sonido aerodinámico . . . . . . . . . . 135 
4.3.1.6 Análisis crítico de la analogía de Lighthill . . . 139

4.3.2 Métodos de resolución de la ecuación de ondas : Función de Green . . . . . . . . . . . . . . . . . . . 141

4.3.3 Descomposición del campo de velocidades del fluido . . 143

4.4 Estudios experimentales en aeroacústica . . . . . . . . . 145

4.5 Análisis experimental del ruido de flujo generado por geometrías simples . . . . . . . . . . . . . . . . . . . 148

4.5.1 Análisis de las leyes de escalado . . . . . . . . . . . . . 162

5 Estudio computacional $\quad 177$

5.1 Introducción . . . . . . . . . . . . . . . . . . . . . . . . 179

5.2 Métodos computacionales: Usos y métodos . . . . . . . . . . 180

5.2.1 Estudio computacional de las teorías acústicas . . . . . 181

5.2 .2 Simulación numérica directa . . . . . . . . . . . . . . 183

5.2.2.1 Condiciones de contorno para fluidos no estacionarios . . . . . . . . . . . . 184

5.2.2.2 Selección del esquema de diferencias finítas . . 185

5.2.3 Simulación numérica directa más cálculo acústico . . . 186

5.2.4 Efectos turbulentos . . . . . . . . . . . . . . 188

5.3 Simulaciones numéricas de los campos turbulentos . . . . . . 189

5.3.1 Determinación de campos de velocidades estocásticos . . 189

5.3.2 Modelos de turbulencia de dos escalas . . . . . . . . . . 191

5.3.3 Representación de flujos turbulentos mediante la generación de señales no gausianas . . . . . . . . . . . . 192

5.4 Cálculos acústicos mediante métodos numéricos . . . . . . . . 193

5.5 Estudio computacional del ruido de flujo generado por silenciadores . . . . . . . . . . . . . . . . . 197

5.5.1 Cálculo de la presión acústica radiada . . . . . . . . 200

5.5.2 Estimación de la intensidad sonora global transmitida al campo libre . . . . . . . . . . . . 206

5.6 Resultados de la aplicación a una geometría simple . . . . . . . 207 
6 Conclusiones y desarrollos futuros

6.1 Conclusiones . . . . . . . . . . . . . . . . . . 223

6.2 Desarrollos Futuros . . . . . . . . . . . . . . . . . . . 231

A Analogías acústicas $\quad 233$

A.1 Teoría de la generación de ruido por vórtices de Powell . . . . . 235

A.2 Contribución de Howe al ruido aerodinámico . . . . . . . . 237

A.3 Teoría acústica de Ribner . . . . . . . . . . . . . . . . . . . . 239

A.4 Generalización acústica propuesta por Doak . . . . . . . . . . 240

A.5 Ecuación de Lilley . . . . . . . . . . . . . . . . . . . . . 244

B Métodos de resolución de ecuaciones de ondas 247

B.1 Método de Kirchhoff . . . . . . . . . . . . . . . . . . . . . . . . 249

B.2 Ecuaciones de Ffowcs Williams y Hawkings . . . . . . . . 250

B.3 Variables de semejanza . . . . . . . . . . . . . . . . 252

C Análisis de errores de los sistemas de medida 253

C.1 Características de los sensores . . . . . . . . . . . . . 255

C.1.1 Transductores de medida de presión en cánula . . . . 255

C.1.2 Micrófonos empleados para la medida de presión en conductos . . . . . . . . . . . . . 257

C.1.3 Sondas de intensidad . . . . . . . . . . . . . 258

C.1.3.1 Errores de medida . . . . . . . . . . . . . 259

C.1.3.2 Influencia de la posición de medida de intensidad261

C.2 Sistemas de calibración . . . . . . . . . . . . . . . . . 263

C.2.1 Calibración de los transductores de presión . . . . . . 263

C.2.2 Método de calibración de los micrófonos de medida de presión en conductos . . . . . . . . . . . . . . . . 264

C.2.3 Calibración de la sonda de intensidad . . . . . . . . . 264

C.3 Características de la cámara semianecoica . . . . . . . . . . . 272 


\title{
Tabla de símbolos
}

\author{
Latinos
}

$\begin{array}{ll}a & \text { radio de la cánula } \\ a_{o} & \text { velocidad del sonido a temperatura ambiente } \\ A_{s} & \text { sección de la cánula } \\ \mathbf{a}(\theta) & \text { vector respuesta del array o de dirección de la señal } \\ \mathbf{A}(\Theta) & \text { matriz de restricciones } \\ \mathbf{b}_{a t} & \text { componente acústica y térmica del momento lineal } \rho \mathbf{u} \\ \mathbf{b}_{t} & \text { componente turbulenta del momento lineal } \rho \mathbf{u} \\ c & \text { velocidad del sonido a la temperatura del flujo } \\ C & \text { capacidad de un condensador } \\ c_{K} & \text { constante de error del índice de presión-intensidad } \\ c_{p} & \text { calor específico a presión constante } \\ d & \text { distancia entre los captadores de presión en la cánula } \\ d_{c} & \text { distancia entre las cánulas de entrada y salida de un silenciador } \\ d_{i} & \text { diámetro de la cánula de entrada de un silenciador } \\ d_{o} & \text { diámetro de la cánula de salida de un silenciador } \\ d_{n} & \text { distancia del sensor n-ésimo al primer sensor de presión en la cánula } \\ d \mathcal{S} & \text { diferencial de superficie } \\ D(\omega, \theta) & \text { factor de directividad del modelo de emisión } \\ D / D t & \text { derivada convectiva }\left(D / D t \equiv \partial / \partial t+u_{i} \cdot \partial / \partial x_{i}\right) \\ E(.) & \text { valor esperado de una magnitud } \\ e_{\phi} & \text { error debido al desfase entre los micrófonos de la sonda de intensidad } \\ e_{s o n d a} & \text { error debido al espaciado entre los micrófonos de la sonda de intensidad } \\ \mathbf{F}_{r} & \text { fuerza de resistencia aerodinámica } \\ \mathbf{f} & \text { fuerza por unidad de volumen } \\ f & \text { frecuencia lineal } \\ f_{c} & \text { frecuencia característica de una cánula } \\ f_{i} & \text { función del número de Helmholtz presente en el coeficiente de reflexión } \\ f_{k} & \\ f_{o}(k a) & \end{array}$


$f(\mathbf{r}, t) \quad$ función que representa el campo acústico

$f(k, \theta) \quad$ factor de distribución angular de la presión radiada

$f(M) \quad$ función de dependencia del coeficiente de reflexión con el flujo medio

$f_{s}$

$f_{\text {onda }}$

Frecuencia de muestreo espacial de una señal

$g$

Frecuencia característica de una onda

ganancia de la respuesta de la salida del beamformer de cualquier señal procedente de la dirección y frecuencia especificadas

g vector de la respuesta deseada en el algoritmo de descomposición

$G(\mathbf{r}, t) \quad$ función de Green espacio temporal

$G_{\omega}\left(\mathbf{r} \mid \mathbf{r}_{0}\right) \quad$ Transformada de Fourier de $G(\mathbf{r}, t)$

$G_{f} \quad$ región confinada donde se encuentra la fuente

$G_{x y} \quad$ espectro cruzado de los micrófonos x e y

$G_{x x} \quad$ autoespectro del micrófono x

$H \quad$ función de Heaviside

$\mathcal{H}(t) \quad$ Matriz de transferencia

$H_{m} \quad$ armónico m-ésimo del motor $\left(H_{m}=m \times\right.$ régimen de giro $\left./ 60\right)$

$H(\theta, f) \quad$ función respuesta del beamformer

$h \quad$ entalpía específica del fluido

$h^{\prime} \quad$ fluctuación de la entalpía específica, $h$

$h_{o} \quad$ entalpía de parada $\left(h_{o}=h+1 / 2 \cdot u^{2}\right)$

$h_{o}^{\prime} \quad$ fluctuación de la entalpía de parada

$H e=k \cdot a \quad$ número de Helmholtz

I

$I_{O}$

$I_{T}$

$j=\sqrt{-1}$

$J_{n}$

flujo de energía por unidad de longitud o intensidad acústica

$k=\omega / c$

$K=c / a_{o}$

$\mathrm{k}$

$\mathrm{K}_{l}$

$\mathrm{K}_{\mathrm{TT}}$

$\ell$

$L_{c}$ intensidad de referencia $I_{o}=10^{-12} \mathrm{~W} \cdot \mathrm{m}^{-2}$

intensidad total radiada

unidad imaginaria

función de Bessel de orden $n$

número de onda del flujo

razón entre las velocidades del sonido del flujo y ambiente

energía cinética turbulenta

coeficiente de pérdida de carga producido por una lámina

coeficiente de pérdida de carga de una cámara de expansión reversa

longitud de escala característica del movimiento turbulento

$L_{e}$

longitud de una cámara de expansión reversa

$L_{I_{\text {ref }}}$

$L_{t}$

longitud efectiva de una cánula

nivel de intensidad sonora global de referencia

longitud física de una cánula

$l_{M}(\omega)$

corrección de longitud en el extremo abierto modificada con el flujo medio

$l_{o}(\omega)$ corrección de longitud en el extremo abierto en ausencia de flujo medio distancia desde el punto de medida hasta el extremo de la cánula 


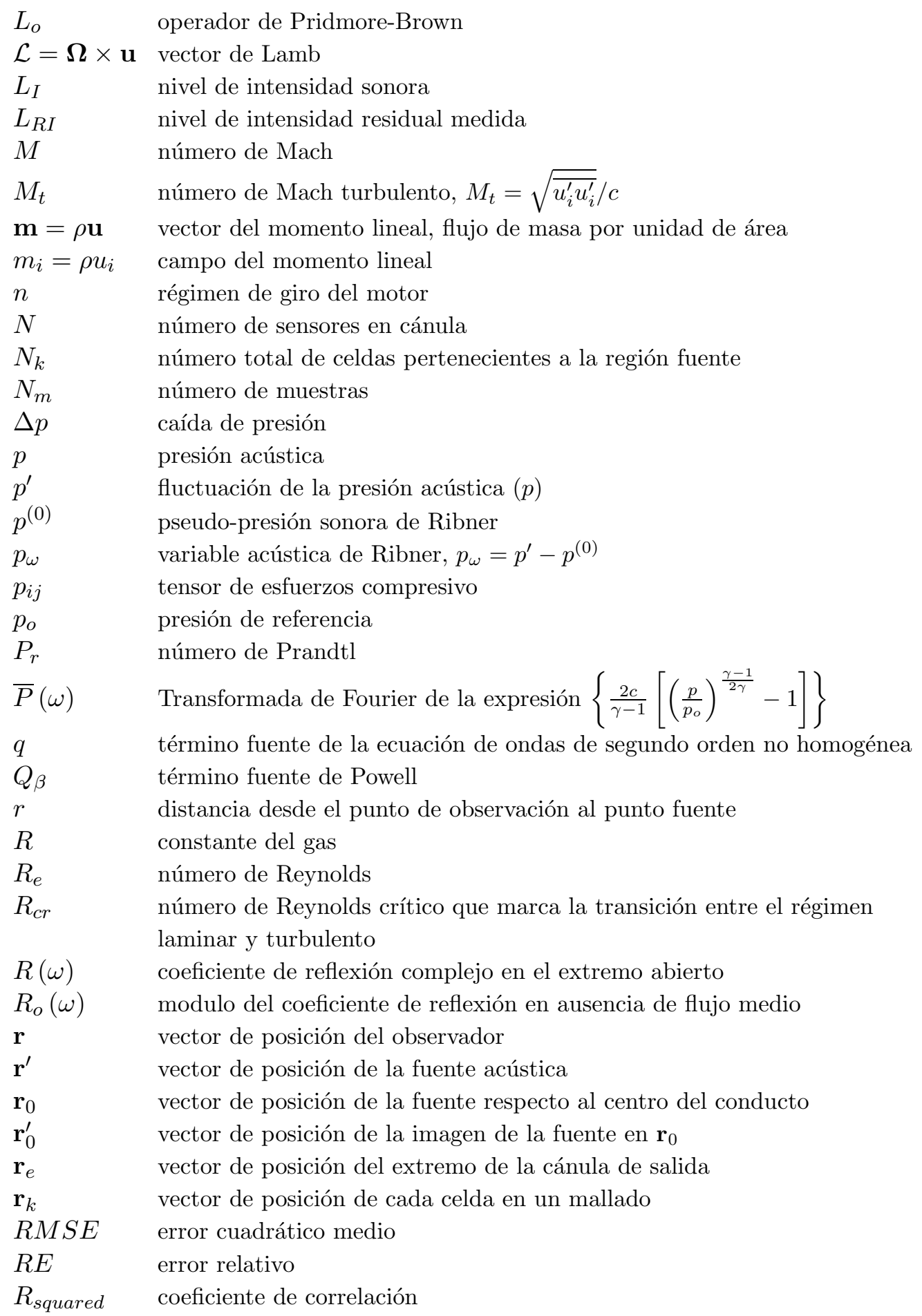


$s$

$\mathbf{u}_{s}$

$\mathbf{u}_{z}$

$v=A_{s} \cdot u$ $\mathrm{V}$ entropía especifica

fluctuación de la entropía

señal fuente

número de Strouhal $(k a / M)$

número de Strouhal acústico $(\omega a /\langle\widetilde{u}(t)\rangle)$

nivel de presión sonora

diferencia de los niveles de presión sonora

instante temporal de la señal

tiempo retardado, $t^{\prime} \equiv t-\left|\mathbf{r}-\mathbf{r}^{\prime}\right| / a_{o}$

temperatura absoluta del gas en la cánula de escape

temperatura de referencia, $293.16 K$

periodo de muestreo espacial

temperatura ambiente

Tensor de esfuerzos de Lighthill

fluctuación de la velocidad total

fluctuación de velocidad total en la boca del escape

aceleración de la fluctuación del volumen del fluido en la boca del escape velocidad media característica del flujo turbulento

velocidad de convección

componentes del campo de velocidades $\mathbf{u}$

componentes fluctuantes del campo de velocidades del flujo turbulento componentes del campo de velocidades corregido con la energía cinética turbulenta $\left(u_{i}^{\prime \prime}=u_{i} \cdot \sqrt{2 \cdot \mathrm{k} /|\mathbf{u}|^{2}}\right)$

componentes medias del campo de velocidades del flujo turbulento campo de velocidades del fluido componente irrotacional del campo de velocidad componente solenoidal del campo de velocidad velocidad de un movimiento armónico simple

fluctuación del caudal volumétrico

componente de la perturbación acústica

derivada temporal de la fluctuación del caudal volumétrico

estimación de la derivada temporal de la fluctuación del caudal volumétrico volumen de la región turbulenta

suma de las aceleraciones

peso de la señal de salida del transductor n-ésimo

potencia acústica

vector de pesos del beamformer

componentes de dirección

vector de salida del sensor en cánula 
$\mathbf{X}\left(f_{k}\right) \quad$ componente k-ésima de la transforma de Fourier discreta de $\mathbf{x}(t)$

$x_{n}(t) \quad$ señal instantánea del sensor n-ésimo

$y(t) \quad$ señal de salida del beamformer en el instante $t$

$\mathbf{Y}\left(f_{k}\right) \quad$ componente k-ésima de la transforma de Fourier discreta de $y(t)$

$Z(\omega) \quad$ impedancia de la superficie

\section{Griegos}

$\alpha$

$\alpha_{s}$

$\beta$

$\beta_{s}$

$\gamma$

$\gamma_{x y}$

$\delta_{i j}$

$\delta_{p I}$

$\delta_{p I o}$

$\delta V$

$\eta$

$\epsilon$

$\varepsilon$

$\varepsilon_{s t}$

$\zeta$

$\theta$

$\lambda$

$\nu$

$\nu_{k}$

$\mu$

$\mu^{\prime}$

$\mu_{t}$

$\xi$

$\pi_{o}$

$\rho$

$\rho^{\prime}$

$\rho_{o}$

$\rho_{j}$

$(\mathfrak{r}, \theta, \phi)$

$\tau$

coeficiente de atenuación viscotérmico

factor de restricción acústica del flujo

número de onda que incluye los efectos de disipación viscotérmica

admitancia específica de la superficie

cociente de los calores específicos

coherencia entre los registros de dos sensores $x$ e $y$

delta de Kronecker

índice de presión-intensidad

índice de presión-intensidad residual

elemento de volumen

variable del campo acústico definida como $\eta \equiv \ln \left(p / p_{o}\right)$

desplazamiento infinitesimal

razón de disipación turbulenta

error estándar de la correlación

valor medio del cociente entre la fluctuación de la velocidad en el extremo

del conducto y la velocidad media del flujo $(\langle\widetilde{u}(t) / U\rangle)$

ángulo polar

longitud de onda de la señal

viscosidad cinemática

elemento de volumen finito e infenitesimal de un mallado

viscosidad dinámica

viscosidad de volumen (bulk viscosity)

coeficiente de viscosidad turbulenta

$\xi \equiv \log \left(L_{c} / d_{o}\right)$

polo de inestabilidad de la onda de presión reflejada

densidad del medio

fluctuación de la densidad

densidad a temperatura ambiente

densidad a la temperatura del fluido

coordenadas esféricas

intervalo de promedio temporal 


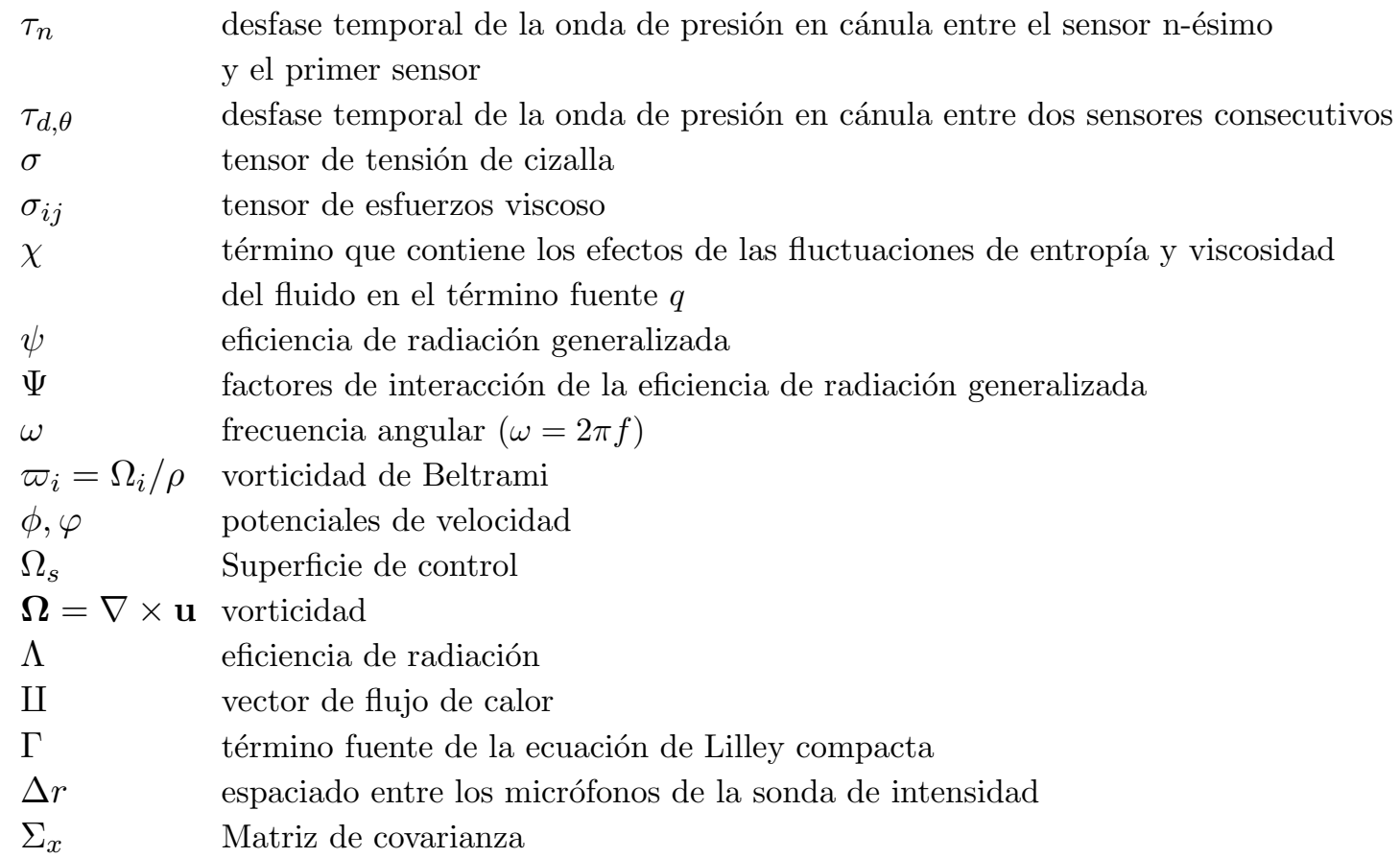

\section{Subindices y superíndices}

$\begin{array}{ll}+ & \text { Componente progresiva } \\ - & \text { Componente regresiva } \\ 1,2,3 & \text { Puntos de medida } \\ d & \text { Disipado } \\ K & \text { Imposición de la condición de Kutta } \\ M & \text { Correcciones en presencia de flujo medio } \\ N K & \text { No imposición de la condición de Kutta } \\ r & \text { Radiado } \\ r e f & \text { Condiciones de referencia } \\ r m s & \text { Valor eficaz de la magnitud considerada } \\ t & \text { Transmitido } \\ T T & \text { Cámara de expansión reversa } \\ x & \text { Proyección sobre el eje } X \text { (de un sistema de coordenadas cartesianas) } \\ y & \text { Proyección sobre el eje } Y \text { (de un sistema de coordenadas cartesianas) } \\ z & \text { Proyección sobre el eje } Z \text { (de un sistema de coordenadas cartesianas) }\end{array}$


Siglas

SPL Sound Pressure Level

CFD Computational Fluid Dynamics

DNS Direct Numerical Simulation

LES Large Eddy Simulation

DNSC Direct Numerical Simulation of unsteady Compressible fluid flows

EEL Ecuaciones de Euler Linealizadas

SNRG Stochastic Noiseand Radiation Generation model

RANS Reynolds-Average Navier-Stokes equations

RMSE Root Mean Squared Error

RE Relative Error

\section{Operadores}

$\begin{array}{ll}\nabla & \text { Operador Nabla } \nabla=\frac{\partial}{\partial \mathrm{x}} \mathbf{i}+\frac{\partial}{\partial \mathrm{y}} \mathbf{j}+\frac{\partial}{\partial \mathrm{z}} \mathbf{k} \\ \nabla^{2} \equiv \Delta & \text { Operador Laplaciano } \nabla^{2}=\frac{\partial^{2}}{\partial \mathrm{x}^{2}}+\frac{\partial^{2}}{\partial \mathrm{y}^{2}}+\frac{\partial^{2}}{\partial \mathrm{z}^{2}} \\ (.)^{H} & \text { transpuesta conjugada } \\ (.)^{*} & \text { conjugado } \\ (.)^{T} & \text { transpuesta } \\ \langle.\rangle & \text { promediado temporal }\end{array}$





\section{Capítulo 1}

\section{Introducción}

1.1 Introducción $\ldots \ldots \ldots \ldots \ldots$

1.2 Fuentes de ruido en el motor . . . . . . . . 3

1.3 Objetivos y desarrollo del trabajo ....... 10 



\section{$1.1 \quad$ Introducción}

En el presente capítulo se pretende proporcionar una visión global del ruido emitido por motores de combustión interna alternativos, así como de la problemática particular de la predicción del ruido emitido en el proceso de escape. Con tal fin, en primer lugar se realizará una descripción de los diferentes mecanismos por los que un motor puede radiar ruido, resaltando la importancia del estudio del ruido aerodinámico provocado por el proceso de renovación de la carga, y en particular del ruido emitido durante el proceso de escape, haciendo especial hincapié en los mecanismos de generación sonora en este último proceso, con el objetivo de situar adecuadamente el ruido de flujo, en cuanto a sus características y relevancia, ya que su estudio y predicción es el objetivo de la presente tesis.

En segundo lugar nos centraremos en la problemática de la predicción de la contribución del ruido de flujo al ruido de escape finalmente emitido, desde el punto de vista de la descripción de los mecanismos de su generación y de su respuesta espectral. A continuación, y como paso previo a la descripción del núcleo del trabajo presentado, se mencionarán las ventajas e insuficiencias de los diferentes procedimientos propuestos en la literatura, sirviéndonos como marco del procedimiento propuesto y como guía del mismo.

\subsection{Fuentes de ruido en el motor}

El problema de la emisión de ruido por vehículos a motor ha adquirido en los últimos años una especial relevancia, debido principalmente a dos causas:

- La creciente sensibilización hacia los fenómenos de contaminación ambiental con implicaciones en la salud pública [159], entre ellos el de contaminación acústica en relación a las emisiones de ruido de intensidad elevada por los vehículos. Esto se traduce en la elaboración y exigencia de cumplimiento de normativas cada vez más restrictivas [122] sobre los niveles de emisión autorizados (normativa 92/97/EEC) [98].

- El aumento de las exigencias del mercado en cuanto a vehículos que posean mayores prestaciones a la vez que dispongan de habitáculos más confortables, lo que engloba la creciente preocupación por el confort acústico y conlleva la necesidad de desarrollar vehículos cada vez más silenciosos [85]. 
A la hora de dar respuesta a estas necesidades hay que tener en cuenta las restricciones adicionales a las que debe ceñirse el diseño de los sistemas orientados a la disminución de las emisiones de ruido:

- Disponibilidad espacial para la instalación

- Aumento de la potencia del motor.

- Necesidad de obtener un balance de costes positivo para el fabricante que englobe factores de durabilidad, peso, etc.

Parece evidente que el proceso global de generación de ruido por un vehículo es un proceso sumamente complejo. En él coexisten diferentes fuentes tales como el motor de combustión interna alternativo, la interacción con el aire circundante, la contribución del sistema motriz, y la interacción de las ruedas con el firme que se transmiten al habitáculo a través de la suspensión [74]. En este trabajo nos centraremos en el estudio de la contribución del motor, que ya es en sí un problema suficientemente complejo, eliminando de este modo, contribuciones como la del flujo alrededor del cuerpo del automóvil, que debido a las altas velocidades que adquiere el flujo es considerada como una de las fuentes principales del ruido del tráfico.

Es obvio que para reducir el ruido emitido por una fuente sonora, en este caso el motor, es necesario analizarla, aislar sus diferentes componentes y establecer la importancia relativa de cada una de ellas dentro del ruido global emitido. Este análisis permitiría elaborar criterios de acción a fin de reducir la potencia sonora emitida, objetivo primordial en el diseño de cualquier sistema de escape. La importancia de la separación de fuentes radica en su bien conocido comportamiento de suma logarítmica, lo cual permite establecer una clara jerarquía entre ellas y analizar si existen algunas claramente dominantes [35], si bien es cierto que, a medida que se reducen los límites legales de emisión permitidos, contribuciones al ruido generado antes poco importantes se convierten en fuentes sonoras cada vez más relevantes. Por ello, en la identificación de las fuentes existen al menos dos tareas igualmente fundamentales como son: (i) la de reconocer qué fuentes son responsables de que el sistema pase o no la normativa establecida, fuentes dominantes en relación a su amplitud sonora, y (ii) la de asociar las fuentes sonoras al espectro obtenido, analizando de este modo no sólo su amplitud sino también su frecuencia con el objetivo de establecer el carácter más o menos molesto de dichas componentes.

En la literatura se recogen diferentes métodos para llevar a cabo esta descomposición, los cuales se diferencian en coste, eficacia y complejidad. Uno 
de los más comúnmente empleados es el que podría llamarse de aislamiento selectivo, [167], y que consiste en comparar el ruido emitido por el motor, cuando este se encuentra encapsulado por un aislante acústico, y el registrado cuando determinadas porciones del motor son descubiertas. Los inconvenientes principales de este método son su coste en tiempo de banco de ensayos, debido a la complejidad del ensayo, y a la dispersión resultante de los ensayos al no ser estos totalmente repetitivos. Además, se debe tener en cuenta que en muchas ocasiones, para que pueda extraerse la máxima información posible, estos ensayos deben acompañarse de medidas adicionales del ruido generado por el motor sin aislar, lo cual todavía le confiere un mayor coste.

Con el objetivo de abaratar costes y de proporcionar mayor información sobre los mecanismos de generación de ruido, se han desarrollado nuevos métodos basados en el empleo de técnicas centradas en el análisis de Fourier. Estas técnicas se aplican en general a medidas de intensimetría acústica $[119,148,166]$ (medidas realizadas con antenas focalizadas sobre las diferentes fuentes bajo estudio), o bien medidas directas de vibración mediante acelerómetros [179]. Además de estas técnicas, en la actualidad se está aumentando el empleo de técnicas de análisis espectral tiempo-frecuencia, como transformadas de Wavelets [53], de Hilbert o incluso el uso de distribuciones de Wigner [74]. Estas últimas técnicas, permiten obtener una mayor información sobre los mecanismos de generación sonora, ya que, dan información no sólo de su característica espectral (amplitud, frecuencia), sino también de su comportamiento en el tiempo: estacionario o transitorio.

Los resultados de cualquiera de estas técnicas de separación de fuentes dejan patente la dificultad tanto de su identificación como del intento de establecer su importancia relativa, ya que el efecto y contribución de las mismas depende no sólo de su naturaleza sino también de las características del motor considerado [144], relacionados tanto con aspectos constructivos del mismo (como por ejemplo, cilindros en línea o en V), como con sus condiciones de uso (motores pequeños o grandes motores industriales). Además teniendo en cuenta que el ruido es por definición un sonido no deseado, a los factores mencionados debemos añadir como dificultad adicional la referencia necesaria a su carácter subjetivo, pudiendo convertir contribuciones cuantitativamente pequeñas al ruido total emitido en factores de diseño importantes [154].

Tras estos comentarios parece difícil inferir con precisión cuales son las diferentes fuentes presentes en la emisión sonora por el motor y establecer una jerarquía clara entre ellas. Sin embargo, los resultados recogidos en la literatura permiten establecer distintas clasificaciones, expuestas a continuación, a través de las cuales podremos centrar el tema objeto de este estudio. 
En primer lugar, atendiendo al fenómeno responsable de la generación de ruido podemos distinguir las siguientes fuentes:

- Ruido debido al proceso de renovación de la carga.

- Ruido generado en el proceso de combustión.

- Ruido mecánico.

A estas contribuciones, procedentes del motor mismo, deben añadirse aquellas originadas por distintos elementos auxiliares (ventilador, etc.). En los motores Diesel debe considerarse además el ruido de inyección originado tanto por el funcionamiento de la bomba [122] como por el propio proceso de la inyección [180].

Una vez vistos cuales son los fenómenos físicos responsables de la generación de ruido, es necesario tener un buen conocimiento de los mecanismos físicos envueltos en estos procesos, es decir, conocer cómo se producen. Bajo este punto de vista la clasificación del ruido generado en función del mecanismo físico al que está asociado viene dada por [165]:

- Ruido aerodinámico, causado por fluctuaciones no estacionarias de caudal en los extremos de los colectores de admisión y escape.

- Radiación por superficies.

El primero de estos dos mecanismos está asociado al ruido de admisión y escape, cuyo origen se encuentra en el proceso de renovación de la carga, y en el cual se puede distinguir [51] el originado por el propio proceso de llenado y vaciado de los cilindros (ruido pulsante) y el ruido debido a la generación de la turbulencia (ruido de flujo).

El ruido de comportamiento pulsante está directamente asociado con el flujo cuasiperiódico inducido, tanto en el sistema de admisión y escape, por el proceso de intercambio gaseoso en los cilindros que se genera durante la apertura y cierre de las válvulas, de manera que las ondas de presión se propagan a través de los colectores reflejándose en el extremo de los mismos e interfiriendo unas con otras. Esto le confiere la característica de presentar un espectro bien ordenado, con la mayor parte de la energía concentrada en los múltiplos de la frecuencia de encendido del motor [52], dependiendo directamente de su régimen de giro. Este mecanismo de generación sonora es clasificado por Davies [46] como la fuente principal del ruido de escape emitido. 
La forma del pulso de presión inducido por los fenómenos que ocurren en el cilindro (combustión, renovación de la carga, etc.), relacionada directamente con la ley de apertura, se convierte en un factor de primer orden ya que determina en gran medida el contenido espectral del ruido pulsante. Por esta razón, como las variaciones de presión en la válvula de admisión son relativamente suaves la emisión predominante debida al proceso de admisión es de baja frecuencia [94], mientras que en el proceso de escape debido a la mayor amplitud y brusquedad de la variación de presión asociada a la descarga (pudiendo llegar a desarrollarse ondas de choque en el colector a altos regímenes de giro [155]), se produce ruido de alta frecuencia, generando un ruido de escape de gran amplitud y carácter molesto. Más acusado es el frente de ondas de presión generado por un motor de dos tiempos y mayor la contribución de componentes de alta frecuencia a su espectro sonoro. Esto es debido a una apertura más rápida de las lumbreras que en el caso de la apertura de válvulas en un motor de cuatro tiempos [17]. Por tanto, la forma espectral del ruido depende en gran medida de la geometría de la lumbrera, factor que puede ser modificado dentro de un margen relativamente amplio, al contrario que en el caso de las leyes de levantamiento de válvulas en los motores de cuatro tiempos. De modo, que la forma del pulso de descarga del cilindro en motores de dos tiempos adquiere gran relevancia y por tanto se actúa sobre él, mientras que en el caso de motores de cuatro tiempos no se podrá actuar sobre estas fuentes, y se tomarán como parámetros de diseño otros puntos del sistema.

Por otro lado, se consideran [46] fuentes de ruido secundarias aquellas relacionadas con los diferentes mecanismos de generación de turbulencia (ruido de flujo), que podemos dividir en dos grandes grupos:

- La turbulencia generada por la emisión no estacionaria de gases calientes a la atmósfera durante el proceso de descarga en la boca del colector, resultado de la interacción entre las perturbaciones de presión existentes en dicha sección y la atmósfera exterior en reposo, factor que está directamente asociado con la propagación en el espacio libre.

- Turbulencia generada en las distintas singularidades que atraviesa el flujo en el interior del sistema, tanto de admisión como de escape, singularidades que llevan asociadas irreversibilidades e inestabilidades del flujo y por tanto, patrones complejos del mismo.

El primer mecanismo de generación de ruido de flujo por el sistema de escape (emisión no estacionaría a la atmósfera), es claramente ineludible y por tanto la única posibilidad de reducirlo es haciendo una adecuada elección de 
los parámetros que definen este proceso. Con respecto a la segunda fuente de sonido aerodinámico, podría esperarse que el sonido generado en aquellas partes del sistema de escape próximas al motor no alcanzaran, en general, la boca de escape, y pudieran ser atenuadas por el escape mismo [52]. En este sentido, como consecuencia de las velocidades del flujo en las válvulas, que son considerablemente mayores que a la salida de la cánula, y de los fenómenos de desprendimiento de vena fluida a aperturas parciales de las mismas, gran parte de la turbulencia se crea justo aguas abajo de las válvulas [177]. Asimismo, son también fuentes de ruido de flujo los mecanismos de turbulencia inducidos por el flujo asociados con capas límites, separación de flujos, estelas y generación de trenes de vórtices ordenados, que tienen lugar en los cambios bruscos de sección (contracciones y expansiones), curvaturas, válvulas de mariposa [10] y otras discontinuidades distribuidas a lo largo del sistema [49].

Cabe señalar, que las diferencias acústicas existentes entre el ruido pulsante y el ruido de flujo quedan bien reflejadas en sus comportamientos espectrales. Así, el carácter turbulento de este último hace que la energía acústica resultante se presente tanto en tonos discretos relacionados con las resonancias acústicas de la cánula, como distribuida en una banda ancha de frecuencia [38]. Por tanto, no presentará una dependencia clara con el régimen de giro, sino un espectro esencialmente continuo para el intervalo de frecuencias asociado a la evolución de los torbellinos turbulentos.

La contribución de la generación de emisión sonora debida a los fenómenos turbulentos internos, se debe considerar bajo dos perspectivas diferentes. Por un lado, parece obvio esperar que únicamente una pequeña parte de los mismos contribuya al sonido final emitido debido a la disipación de dicha turbulencia en el seno del fluido. Por otro lado, deben tenerse en cuenta los efectos de acoplamiento que presenta el flujo turbulento sobre las características acústicas del sistema pudiendo amplificar determinadas resonancias acústicas. De modo, que esta resonancia inducida por el flujo puede ser un generador de sonido coherente, mientras que mecanismos similares pueden proporcionar una amplificación selectiva de la excitación acústica existente [48], constituyéndose así como fuentes adicionales de ruido [38], o desplazar las fuentes principales [46].

En resumen, la parte de energía del campo turbulento que sea transferida al campo acústico como ondas de presión debe considerarse como una fuente adicional, cuya influencia en el espectro finalmente radiado es claramente dependiente de la geometría del sistema que atraviesa el flujo. Este fenómeno puede ser particularmente importante en el caso de la turbulencia generada por el último silenciador y la cánula de escape en sí misma [52], por su proximidad relativa a la boca de salida. 
En lo que respecta a la turbulencia generada durante el proceso de descarga del pulso de presión en la boca de salida, ésta presenta una contribución sustancial al ruido aerodinámico generado sobre todo a altos regímenes de giro, ya que en estos caso la velocidad media de dicho flujo es de magnitud considerable [17]. Por otro lado, a esta generación sonora contribuye la interacción existente entre el flujo subexpandido en esta sección y los anillos vorticales generados en su entorno [74]. Este fenómeno presenta cierta complejidad debido a su carácter de patrón de flujo no estacionario, caso en el que no resulta trivial separar su contribución de la correspondiente a las propias fluctuaciones del flujo. Sin embargo, a altos regímenes de giro cabe esperar que su comportamiento sea similar al que se manifiesta en condiciones estacionarias [135].

Así como el ruido aerodinámico es debido esencialmente a fenómenos asociados al proceso de renovación de la carga, el segundo mecanismo citado antes, es decir, la radiación por superficies, está asociado al ruido radiado desde las superficies del motor y se origina por el motor en sí mismo, siendo sus tres fuentes principales: la renovación de la carga, la combustión y el ruido mecánico. Este tipo de ruido se genera por la transmisión de las vibraciones del motor hacia las partes mecánicamente conectadas al mismo, desde las cuales se radia al medio ambiente [165]. Así por ejemplo, en el caso de renovación de la carga este proceso tiene lugar a través de las paredes del colector que vibran [34] debido al flujo que se desarrolla en su interior. Esta vibración puede ser particularmente importante cuando alguna frecuencia de excitación debida al flujo, o a su turbulencia, se acopla con las frecuencias características de vibración mecánica del colector [60]. Estos fenómenos pueden llegar a ser determinantes cuando el acoplamiento tiene lugar a baja frecuencia, caso en el que pueden excitarse las frecuencias mecánicas propias de la carrocería y hacer inviable la permanencia en el habitáculo [30]. De aquí se deriva la importancia de establecer criterios constructivos de estos colectores a fin de controlar sus frecuencias características.

Por su parte, tanto el ruido de combustión como el ruido mecánico se radian a través del bloque motor, de modo que será relevante el análisis y optimización de los modos de vibración de la estructura [144], ya que son estos los que determinarán en última estancia la mayor contribución al contenido espectral del ruido emitido [148].

Sin embargo los mecanismos de excitación en ambos casos son radicalmente distintos. En el caso del ruido de combustión éste proviene de la evolución de la presión en el interior del cilindro, siendo el factor que lo determina la brusca subida que experimenta en el inicio de la combustión. También puede 
considerarse una componente turbulenta en el ruido de combustión, aunque de magnitud considerablemente inferior [27] a la generada por el pulso de presión, lo que permite asociar el ruido de combustión a la ley de liberación de calor, existiendo estudios de optimización de ésta en relación al ruido generado [151].

Por el contrario, las fuentes de excitación del ruido mecánico están asociadas al movimiento de las válvulas y al movimiento del pistón que puede considerarse como una de las fuentes principales de ruido mecánico [122]. En particular, el movimiento de cabeceo del pistón dentro del cilindro es uno de los más molestos debido al carácter impulsional de la fuente y su rango frecuencial [122], con el agravante de que éste ha ido adquiriendo mayor importancia a medida que se ha ido reduciendo la contribución de otras fuentes. Un factor que hace especialmente complejo el control de esta fuente de ruido es su clara dependencia con la combustión, de forma que es necesario analizarla separadamente para los diferentes tipos de motores: de encendido provocado y motores diesel, de inyección directa o indirecta.

De entre todas las fuentes citadas, nos centraremos en el estudio del ruido aerodinámico, en particular en el estudio del ruido de flujo generado por los mecanismos de turbulencia y su contribución a la emisión del ruido de escape (ya que como se ha mencionado su contribución al ruido de admisión es un factor poco relevante [49]). Es este un problema suficientemente complejo y relevante, si se tiene en cuenta que lo que se pretende es disminuir el ruido emitido teniendo en mente cualquier efecto negativo que un cambio en la línea de escape puede tener sobre las prestaciones del motor [74].

A pesar de que la componente turbulenta del flujo no es la fuente principal del ruido de escape, como se ha mencionado anteriormente, sino que se constituye como una fuente secundaria, el hecho de que su efecto sea en gran medida desconocido y de que, a medida que se ha ido reduciendo la contribución de la fuente principal (ruido pulsante) su contribución al ruido de escape es cada vez más importante, y que su interacción con la acústica del sistema puede amplificar la contribución de la fuente principal, hace que su estudio sea de gran importancia puesto que su optimización se ha convertido en un criterio de diseño relevante de los sistemas de escape, entre otras razones, por su carácter particularmente molesto debido a su forma espectral con contenido de alta frecuencia.

\subsection{Objetivos y desarrollo del trabajo}

A fin de comprender mejor el tratamiento realizado del ruido de flujo en esta tesis, con el objetivo primordial de poder reducir su contribución, se 
deben tener en cuenta varios factores asociados a su problemática particular. En primer lugar, aunque tradicionalmente el principal objetivo en el diseño de silenciadores de escape para un motor de combustión interna alternativo ha sido la atenuación de la componente pulsante del ruido emitido, como bien analizan El-Sharkawy y El-Chazly [61], ésta componente presenta una contribución dominante a regímenes de giro bajos y medios, en los cuales la amplitud de las fluctuaciones de la velocidad volumétrica en la boca del escape es mayor que la velocidad volumétrica media inducida por la eyección de los gases de combustión [132]. Sin embargo, al aumentar el régimen de giro los efectos del ruido de flujo comienzan a ser más importantes, de manera que a altos regímenes pueden, incluso, convertirse en las fuentes dominantes en el ruido de escape [92]. Esto, junto con el incremento de la velocidad media del flujo en el escape, y por tanto del ruido de flujo, que va asociada a la optimización de los motores y debido a la necesidad de cumplir la legislación sonora cada vez más restrictiva, proporcionan fuertes incentivos para tratar de mejorar la comprensión cuantitativa de la aeroacústica y de otros factores relevantes que influyen en la generación del ruido de flujo.

En segundo lugar, muchos autores consideran que el ruido de escape puede dividirse en dos rangos frecuenciales; la banda de baja frecuencia que unos autores sitúan muy restrictivamente en torno a los $300 \mathrm{~Hz}$ [46] y otros sobre los $600 \mathrm{~Hz}$ [177], banda asociada a los armónicos generados durante el proceso de descarga por la oscilación del gas en el sistema de escape (ruido pulsante). Y el rango de más alta frecuencia, que se debe a la turbulencia creada por la gran velocidad del flujo que atraviesa el sistema de escape y la interacción del mismo con la atmósfera circundante. Esta división justifica el diferente rango frecuencial considerado en el estudio del ruido de escape emitido por un motor, y en el análisis del ruido de flujo emitido por un flujo estacionario.

Otro factor relevante en el cálculo del ruido aerodinámico es, como ya se ha citado, el conocimiento del fenómeno físico que determina la emisión de ruido en el extremo abierto de la cánula, donde existe un acoplamiento entre el flujo presente en dicha sección de salida y las condiciones exteriores, lo que determina la propagación de una onda sonora en el espacio circundante. La solución rigurosa del problema supondría la solución simultánea de las correspondientes ecuaciones para ambos medios imponiendo que las soluciones sean continuas en la superficie de separación [157]. En el caso más general, la ecuación a resolver sería, en los dos medios, la ecuación de Navier para un fluido viscoso compresible [95]. Sin embargo, la condición de contorno natural para la ecuación de Navier en el espacio libre (flujo saliente nulo a distancia infinita de la fuente) hace que esta solución, debido al enorme volumen de cálculo que 
implica, sea inviable desde el punto de vista práctico. Finalmente se puede obtener una solución de la radiación acústica empleando una aproximación numérica en un dominio de cálculo finito; sin embargo, es dificil formarse una idea más física del problema. Además, la condición de radiación de Sommerfeld [89] (radiación uniforme en todas las direcciones del espacio) no sería del todo satisfecha.

Una simplificación al problema puede derivarse de la suposición de que las fluctuaciones del caudal en la boca de la cánula de escape son de tal magnitud, que puede suponerse que el resultado de su interacción con la atmósfera circundante es la propagación de una onda sonora de pequeña amplitud; de esta manera el flujo a grandes distancias (espacio libre) se comporta como una onda esférica saliente. Sin embargo, si el flujo en el conducto tiene un carácter marcadamente no lineal, como es el caso de la cánula de escape, sería preciso acudir a procesos iterativos para alcanzar la convergencia de las soluciones en la boca del colector. Sólo en el caso de que las perturbaciones sean de pequeña amplitud también en el interior del colector, se encuentran en la literatura resultados de la solución simultánea del flujo para la cánula y el exterior $[128,157]$.

Como respuesta a estas dificultades, y con el objetivo de arrojar un poco de luz sobre la física de los procesos involucrados en la emisión de ruido aerodinámico, se analizará en el capítulo 2 el fenómeno de emisión en el sistema de escape de un motor real, considerando que el problema se puede resolver por separado en ambas regiones (fuente interna y emisión a la atmósfera), desacople que es válido únicamente para el rango de bajas frecuencias, en que la presión sonora es aproximadamente uniforme sobre la sección de salida de la cánula [157], que como ya se vio era la fuente principal en el caso en que la fuente pulsante predomina sobre la de flujo. Para ello, se presentará un modelo de diagnóstico experimental de la fluctuación del caudal en la boca de la cánula, y a su vez se analizarán los diferentes mecanismos físicos presentes en el proceso de descarga mediante el establecimiento de un modelo de emisión realista, que incluya entre otros factores la impedancia del extremo abierto, factor necesario para que la hipótesis del desacoplamiento sea válida [157]. De modo, que se podrá estudiar, finalmente, el efecto del flujo sobre la componente pulsante del ruido de escape, analizándose por comparación directa con las medidas experimentales la viabilidad de realizar un análisis estacionario del ruido de flujo emitido en el proceso de escape.

Una vez analizada la contribución del ruido de flujo sobre la componente pulsante y justificado, en el capítulo 2, el comportamiento estacionario del ruido de flujo emitido (hipótesis aceptada por diversos autores [135]), se 
analizará en el capítulo 3 la contribución estacionaria del ruido de flujo generado en el interior de silenciadores; bajo la motivación adicional que supone la existencia de determinados silenciadores comerciales, en general silenciadores reactivos, diseñados para reducir la componente pulsante, que se comportan como fuentes generadoras de ruido de flujo.

Tradicionalmente, la optimización acústica de sistemas de escape se basaba en el análisis frecuencial de la perdida de transmisión o el efecto de inserción para silenciadores sin flujo de aire empleando la teoría acústica existente. Sin embargo, cuando pasan grandes cantidades de flujo por el silenciador, se produce un importante ruido de flujo que es radiado desde el extremo abierto de la cánula de salida del silenciador, ruido que en algunas ocasiones supera el efecto de reducción acústica global del silenciador [91]. En base a su predicción cabe citar los estudios experimentales de autores como Davies [48], donde además existen referencias a los mecanismos de generación del ruido por la unión de tubos y cambios de sección, pero en el caso de silenciadores más complejos se ha focalizado más en la generación del ruido de flujo en sí misma que en las causas y mecanismos de su generación. En nuestro caso particular, aplicaremos al igual que Davies la técnica de intensimetría en conductos al estudio de las contribuciones de las fuentes internas, generación de turbulencia por singularidades del sistema como cambios bruscos de sección, etc., y las fuentes externas, descarga de un flujo estacionario a la atmósfera. Esta técnica permitirá analizar los resultados de la medida interna y la externa, a fin de establecer que fuentes y mecanismos son responsables de la radiación sonora medida en el exterior.

En último lugar, haremos mención al estudio de las emisiones globales que tienen lugar durante el proceso de radiación de ruido de flujo, en las cuales se engloban todos los mecanismos de generación sonora así como todo el rango frecuencial audible $[20 \mathrm{~Hz}, 20 \mathrm{kHz}]$, siendo estos valores a los que se refieren los límites establecidos en la legislación vigente, y por tanto responsables de que un sistema de escape pase o no la normativa. Este estudio, se realiza a través de las analogías acústicas [102] como establece la literatura existente, las cuales permiten establecer el comportamiento de la fuente distinguiéndo entre fuentes monopolares, dipolares o cuadripolares. Estas emisiones globales son analizadas experimental (en el capítulo 4) y computacionalmente (en el capítulo 5), profundizando de este modo en la contribución de las fuentes internas del silenciador, derivando leyes de escalado que establezcan una relación directa entre el ruido emitido y los parámetros geométricos del silenciador. La finalidad de este estudio es la posibilidad de establecer criterios de diseño que satisfagan los compromisos de: 
- la atenuación acústica de los sistemas de escape

- la perdida de carga generada por los mismos

- el ruido de flujo emitido

de forma que cumpla con la legislación y con las expectativas de usuarios y fabricantes: soluciones óptimas de diseño acústico, requerimientos operacionales y factores económicos.

Finalmente en el capítulo 6, se presentará un resumen de las conclusiones obtenidas y los desarrollos futuros propuestos. 


\section{Capítulo 2}

\section{Diagnóstico experimental del ruido de escape}

2.1 Introducción $\ldots \ldots \ldots \ldots \ldots \ldots \ldots$

2.2 Descripción del método . . . . . . . . . . 19

2.3 Estimación de la fluctuación de velocidad en la boca de la cánula de escape . . . . . . . . 20

2.3.1 Coeficiente de reflexión en el extremo abierto . . . 24

2.3.2 Validación numérica . . . . . . . . . . . . . 27

2.3.3 Discusión sobre el error cometido en la estimación del caudal volumétrico . . . . . . . . . . . . 38

2.4 Validación del método con líneas de escape reales: resultados y discusión . . . . . . . . . 4 41

2.4.1 Análisis de la influencia del error del procedimiento propuesto en la estimación del ruido . . . . . . . . . 4 45

2.5 Evaluación del modelo de emisión . . . . . . . 47

2.6 Modelo monopolar . . . . . . . . . . . . . 52

2.7 Modelo de emisión de un pistón vibrante . . . . 54

2.8 Evaluación de los modelos frente a las medidas experimentales ............... 60

2.8 .1 Medida de ruido . . . . . . . . . . . . . . 61

2.8.2 Resultados de la validación . . . . . . . . . . . 64

2.9 Discusión de los resultados . . . . . . . . . 70

2.10 Carácter estacionario del ruido de flujo en escapes 75 



\section{$2.1 \quad$ Introducción}

Cómo ya se mencionó en el primer capítulo, en el análisis espectral del ruido de escape se puede distinguir: (1) un espectro de ruido ordenado con la mayor parte de la energía concentrada en los armónicos de la frecuencia de encendido del motor [47], que corresponde a una secuencia de componentes tonales asociadas con el flujo pulsante existente en el sistema de escape (contribución más importante a baja frecuencia hasta $800 \mathrm{~Hz}$ ), y (2) una segunda componente, que se debe a la contribución de las fuentes de ruido de flujo, cuya característica espectral es que la energía está repartida en una banda ancha de frecuencias (cuya contribución es más importante a partir de 300 $\mathrm{Hz}$ ). Estas fuentes de ruido de flujo provienen, por un lado, de la turbulencia y otros mecanismos aeroacústicos inducidos por el flujo asociados a capas límites, fenómenos de separación de flujos, generación de ondas y vórtices presentes en las expansiones, contracciones, curvaturas y otras discontinuidades que el flujo atraviesa a lo largo del sistema de escape; y por otro lado, del efecto de la descarga de los gases de escape y su interacción con la atmósfera en reposo.

En este capítulo se realiza un análisis experimental del efecto y comportamiento del flujo en los fenómenos responsables de la generación del ruido de escape emitido al campo libre, que como se ha dicho está compuesto de: ruido pulsante y ruido de flujo. En particular, se presenta y discute, en primer lugar, un procedimiento experimental para la determinación de la componente pulsante del ruido de escape, con la finalidad de establecer cuál es el efecto del flujo sobre dicha componente. De este modo, a partir de la estimación de la componente pulsante y por comparación con los espectros del ruido de escape medidos, se justificará al final del capítulo, la posibilidad de analizar separadamente el ruido pulsante y el ruido de flujo, estudiado en un capítulo posterior; bajo la consideración de que esta segunda componente no está asociada al flujo armónico (como se mostrará en este capítulo).

La estimación de la componente pulsante del ruido de escape, se realiza en dos pasos: en primer lugar, se describe un procedimiento de estimación de la fluctuación de velocidad en la sección de descarga, con el propósito de obtener una estimación de la fuente de fluctuación interna (del sistema de escape); y en segundo lugar, esta componente será emitida a la atmósfera haciendo uso de un modelo de emisión realista. Esta forma de aproximación al problema se basa en la hipótesis de desacoplamiento entre los fenómenos en el interior del conducto y su emisión al exterior, hipótesis que puede considerarse como válida para el rango de bajas frecuencias [157] (rango en que la emisión de la componente pulsante tiene mayor contribución), caso en el que la presión es 
aproximadamente uniforme en toda la sección de salida de la cánula. Además, este modo de operar nos permitirá discriminar entre los efectos debidos a la generación interna (fuente, en la boca de salida de la cánula de escape) y efectos de la descarga (emisión), factores que contienen una referencia implícita a la contribución del flujo sobre ellos. Este procedimiento ofrece la ventaja de poder mejorar aisladamente tanto los modelos de emisión como los cálculos del flujo usados para la predicción de ruido, identificando en cada caso qué efecto producen dichas mejoras en la estimación del espectro de ruido medido y el efecto de los fenómenos del flujo sobre la componente pulsante cuando ésta es emitida a la atmósfera.

El método de estimación de la fluctuación de velocidad en la boca del escape se basa en la medida de presión en la cánula de escape, y será validado numérica y experimentalmente, a fin de comprobar los resultados obtenidos y evaluar las hipótesis básicas consideradas. La validación numérica servirá como primera indicación de las ventajas y posibilidades de este nuevo método de predicción; además, permitirá conocer la influencia de los diferentes parámetros y proporcionará un primer pronóstico de los problemas que podrían presentarse en una implementación experimental. A partir de ella se evaluarán los errores asociados al procedimiento propuesto, analizando la influencia que las incertidumbres en los datos experimentales tienen sobre las cantidades calculadas, de donde se obtiene una primera aproximación al límite de precisión de la estimación realizada.

La validación experimental se realizará de forma indirecta comprobando en primer lugar, su consistencia a través del análisis de las tendencias esperables en la fluctuación del caudal volumétrico a diferentes regímenes de giro (disminución de la amplitud y aumento del ruido al aumentar el régimen de giro); y en segundo lugar, su sensibilidad hacia un cambio en la geometría de la línea de escape mediante la correlación de las estimaciones de la diferencia del nivel de presión sonora (factor independiente del modelo de emisión escogido), con el valor obtenido de la medida experimental de la intensidad.

Una vez evaluado el método de estimación de la fluctuación del caudal volumétrico en la sección de salida de la cánula de escape, se estudia la eficacia del modelo de emisión considerado para la estimación del espectro de ruido radiado, correlacionando la medida exterior realizada en condiciones semianecoicas y la estimación para diferentes modelos de emisión, con el objetivo de valorar cuál de las aproximaciones cuantitativas implementadas describe más adecuadamente el comportamiento físico de la emisión sonora durante el proceso de descarga de la componente pulsante a la atmósfera exterior en reposo. De esta forma, se analizará el efecto del flujo sobre la componente pulsante a 
través de la correlación directa de los espectros de ruido estimados y medidos en el exterior.

Finalmente, se estudiará la viabilidad de realizar un análisis estacionario de la componente de ruido de flujo del proceso de escape en un motor real.

\subsection{Descripción del método}

El objetivo, es obtener la estimación de la componente pulsante del ruido de escape de la configuración medida, a partir de la medida de presión instantánea en un punto de la cánula de salida relativamente próximo al extremo abierto de salida de la misma.

Es sabido que uno de los efectos principales derivados de la existencia de un flujo no estacionario en los colectores es la radiación de ruido aerodinámico en el extremo abierto, debido a la interacción entre el flujo y las condiciones exteriores. El factor clave de esta interacción es la fluctuación del flujo en la boca de la cánula de escape inducida por el proceso de escape llevado a cabo en los cilindros del motor; por tanto, esta fluctuación puede ser considerada como la causa de esta emisión sonora, es decir, la fuente sonora. Esta es la razón por la cual la mayor cantidad de esfuerzos invertidos en la predicción del ruido han sido destinados al cálculo de la fluctuación de la velocidad en el extremo abierto, lo que requiere el conocimiento de las condiciones del flujo que atraviesa el sistema de escape. Una vez calculada esta fluctuación el único requisito para obtener el ruido radiado es disponer de un modelo de emisión adecuado; en relación a este hecho, autores como Jones [87] manifiestan las limitaciones de los modelos de emisión más comúnmente empleados.

Planteado así, cualquier desviación observada en la comparación directa entre las medidas de ruido y las estimaciones puede deberse a dos causas: (i) las limitaciones en el cálculo de la fluctuación del flujo en el extremo abierto, y (ii) los errores asociados al uso de modelos de emisión demasiado simplificados (Landau [95]) sobre todo a alto régimen de giro, cuando la generación de ruido de flujo puede ser, según Kunz [93], la componente dominante. Esta dificultad en la determinación precisa del origen de las diferencias entre las estimaciones y medidas hace extremadamente difícil obtener una valoración adecuada del funcionamiento de cualquier modelo de predicción dado.

Como solución a este problema, la determinación experimental de la fluctuación de la velocidad en el extremo abierto, permite disponer de una comparación directa del cálculo del flujo y de un término "fuente fluctuante" (los dos elementos en que se divide el problema de la estimación de fuente 
generadora del ruido) y evaluar aisladamente el modelo de emisión. Sin embargo, los métodos normales de medida existentes, tales como velocimetría láser-Doppler $(L D V)$ y anemometría de hilo caliente, presentan serios problemas, como manifiestan Piñero et al. [138]. En el caso del $L D V$ se deben considerar dos cuestiones. En primer lugar, la necesidad de dopar el flujo para que el sistema de medida pueda detectar el movimiento del gas, lo cual es extremadamente complicado en sistemas de escape, y en segundo lugar, la dificultad de definir la posición de medida a fin de que esta medida no esté relacionada con el complejo patrón de flujo establecido aguas abajo del extremo abierto. Con respecto a la anemometría de hilo caliente, es la extrema sensibilidad de la sonda lo que hace a esta técnica impracticable en sistemas de escape, ya que el sedimento y las agresiones químicas pueden modificar las características del hilo introduciendo incertidumbres considerables en la medida. Además, la presencia de una sonda en el extremo abierto podría modificar las características del flujo de un modo totalmente impredecible.

Como alternativa, en el modelo propuesto la fluctuación de velocidad en la sección de descarga es obtenida directamente de la medida de presión en cánula, ofreciendo la posibilidad de obtener la contribución al ruido radiado debida únicamente a la fuente interna, es decir, a los elementos que configuran el sistema de escape, sin contener los efectos de la descarga del flujo ni factores ambientales, como podrían ser efectos de reflexión, humedad ambiental, dispersión, absorción, etc. A su vez, el hecho de poder separar las fuentes nos permite predecir los efectos que se producirán en el ruido radiado cuando se lleven a cabo cambios en la línea de escape.

\subsection{Estimación de la fluctuación de velocidad en la boca de la cánula de escape}

El método presentado aquí versa sobre la estimación de las fluctuaciones de velocidad a partir del registro de presión en la cánula de escape. Se asumirá, por tanto, el conocimiento de la presión instantánea en un punto localizado a una distancia $L$ del extremo del conducto y la existencia de un flujo unidimensional en el mismo, hipótesis válida únicamente en la cánula de escape, no en las singularidades que puedan presentarse en el colector de escape, de acuerdo con las dimensiones típicas de la misma. Esta presión puede expresarse como suma no lineal de las componentes progresiva $p^{+}(t)$, que se mueve hacia el extremo abierto, y regresiva $p^{-}(t)$ de la presión, que se propagaría en sentido contrario a la anterior. La presión resultante relacionada con estas ondas de interferenciase expresa de la forma siguiente, como manifiestan Bannister y 
Mucklow [7]:

$$
\left(\frac{p}{p_{o}}\right)^{\frac{\gamma-1}{2 \gamma}}=\left(\frac{p^{+}}{p_{o}}\right)^{\frac{\gamma-1}{2 \gamma}}+\left(\frac{p^{-}}{p_{o}}\right)^{\frac{\gamma-1}{2 \gamma}}-1
$$

donde $p_{o}$ es la presión de referencia y $\gamma$ es el cociente de los calores específicos.

Estos valores de las componentes de presión deben ser transferidos a la sección de salida de la cánula de escape. Con ese propósito, se recuerda la relación entre velocidad del flujo y presión para una onda simple, que viene dada por la ecuación de Earnshaw

$$
u= \pm \frac{2 c}{\gamma-1}\left[\left(\frac{p}{p_{o}}\right)^{\frac{\gamma-1}{2 \gamma}}-1\right]
$$

donde $c$ representa la velocidad del sonido en el medio no perturbado. El signo más se refiere a la onda incidente y el signo menos a la onda reflejada. Sí se asume la validez de la hipótesis de onda simple para las dos componentes en el punto de referencia, de acuerdo con las ideas defendidas por Payri et al. [133], pueden escribirse las correspondientes componentes de la velocidad como:

$$
\begin{gathered}
u^{+}=\frac{2 c}{\gamma-1}\left[\left(\frac{p^{+}}{p_{o}}\right)^{\frac{\gamma-1}{2 \gamma}}-1\right] \longrightarrow\left(\frac{p^{+}}{p_{o}}\right)^{\frac{\gamma-1}{2 \gamma}}=1+\frac{\gamma-1}{2} \frac{u^{+}}{c} \\
u^{-}=-\frac{2 c}{\gamma-1}\left[\left(\frac{p^{-}}{p_{o}}\right)^{\frac{\gamma-1}{2 \gamma}}-1\right] \longrightarrow\left(\frac{p^{-}}{p_{o}}\right)^{\frac{\gamma-1}{2 \gamma}}=1-\frac{\gamma-1}{2} \frac{u^{-}}{c}
\end{gathered}
$$

De modo que puede observarse que mientras las componentes de presión se suman de forma no lineal, de acuerdo con la ecuación (2.1), las componentes de la velocidad pueden ser sumadas de forma lineal, ya que aunque no es posible describir el patrón de flujo complejo resultante desde la interferencia de dos ondas, en términos de una simple adición de las magnitudes correspondientes a ambas componentes interferentes de la onda $\left(p^{+}, p^{-}\right)$para cada uno de los puntos de la región, sí que es posible hacer esto en un punto e instante de tiempo en el cual las dos componentes de la onda considerada coinciden [131]. Esto significa que para un instante de tiempo y punto particular en el que las dos componentes de la onda se encuentran, la velocidad total del fluido está dada por:

$$
u=u^{+}+u^{-}
$$

En una situación real no se dispone de los valores de velocidad, y por lo tanto no se pueden aprovechar las ventajas que proporciona este hecho. En 
lugar de ello, si se sustituyen las ecuaciones (2.3) y (2.4) en la ecuación (2.1) se obtiene

$$
\left(\frac{p}{p_{o}}\right)^{\frac{\gamma-1}{2 \gamma}}=1+\frac{\gamma-1}{2 c}\left[u^{+}-u^{-}\right]
$$

donde de nuevo se ha asumido que la presión puede ser expresada como una adición de ondas simples, de modo que se desprecia cualquier interacción no lineal entre las componentes de presión.

Ahora, las componentes de velocidad deben ser transferidas a la sección de salida de la cánula, teniendo un cuidado especial con el hecho de que deberían aplicarse sus valores en el mismo instante de tiempo. Para salvar esta dificultad conviene trasladarse al dominio frecuencia, en el cual estos aspectos son fácilmente manejables. Para ello, se realiza la transformada de Fourier de la ecuación (2.6) obteniendo

$$
u^{+}(\omega)-u^{-}(\omega)=\bar{P}(\omega)
$$

donde $\bar{P}(\omega)$ es la transformada de Fourier del término que se encuentra en el segundo miembro de la ecuación (2.6):

$$
\bar{P}(\omega)=\frac{1}{\sqrt{2 \pi}} \int \frac{2 c}{\gamma-1}\left[\left(\frac{p}{p_{o}}\right)^{\frac{\gamma-1}{2 \gamma}}-1\right] \cdot e^{j \omega t} d t
$$

Una vez llegado a este punto se hace necesario el expresar la ecuación (2.7) como función de las componentes de la velocidad en el extremo abierto. Con este propósito, se considerará la existencia de propagación lineal en el conducto corregida con el modelo de disipación viscotérmica propuesto por Davies [38]. Esta corrección es necesaria debido a la hipótesis de flujo unidimensional en el conducto y el carácter esencialmente tridimensional de los esfuerzos viscosos, que obligan a la introducción de términos ad hoc en las ecuaciones que den cuenta de los efectos disipativos en el flujo. La aproximación de linealidad, se justifica por la consideración de puntos de medida de presión relativamente próximos al extremo abierto. Si las componentes de la velocidad en el extremo abierto las denotamos con una tilde encima, las expresiones obtenidas serán

$$
\begin{gathered}
\widetilde{u}^{+}(\omega)=u^{+} \cdot \exp \left(-j \beta^{+} L\right) \\
\widetilde{u}^{-}(\omega)=u^{-} \cdot \exp \left(j \beta^{-} L\right)
\end{gathered}
$$

donde las expresiones de los números de onda incidente y reflejado apropiados $\beta^{+}$y $\beta^{-}$, que aparecen en las ecuaciones (2.7) y (2.8) vienen dados por

$$
\beta^{+}=\beta /(1+M) \quad y \quad \beta^{-}=\beta /(1-M)
$$


siendo $M=U / c$ el número de Mach del flujo medio, $U$ la velocidad media del flujo y $\beta$ es el número de onda adecuado en ausencia de flujo medio cuando se consideran los efectos viscotérmicos. De acuerdo con Davies [42], su expresión se corresponde con

$$
\beta=k+\alpha(1-j)
$$

donde $k=\omega / c$ es el número de onda acústico y $\alpha$ es el coeficiente de atenuación viscotérmico cuya expresión dada por Lord Rayleigh [145] es

$$
\alpha=(1 / a c) \cdot(\nu \cdot \omega / 2)^{1 / 2}\left(1+[\gamma-1] \cdot P_{r}^{-1 / 2}\right)
$$

donde $\nu$ es la viscosidad cinemática, $a$ es el radio de la cánula y $P_{r}$ es el número de Prandtl del gas en la cánula. La dependencia de la viscosidad cinemática, $\nu$, del aire seco con la temperatura fue dada por Peters et al. [137] como $\nu=A+B \cdot\left(T-T_{r e f}\right)$, donde $T$ representa la temperatura absoluta del aire en el interior de la cánula, $A=1.51 \cdot 10^{-5} \mathrm{~m}^{2} / \mathrm{s}, B=9.2 \cdot 10^{-8} \mathrm{~m}^{2} / \mathrm{s} K$ y $T_{\text {ref }}=293.16 \mathrm{~K}$.

A partir de las ecuaciones (2.9) y (2.10) es posible obtener las componentes incidente y reflejada de la velocidad en el punto de medida en función de sus valores en el extremo abierto y sustituyendo sus valores en la ecuación (2.7) se obtiene

$$
\widetilde{u}^{+}(\omega) \cdot e^{j \beta^{+} L}-\widetilde{u}^{-}(\omega) \cdot e^{-j \beta^{-} L}=\bar{P}(\omega)
$$

Sin embargo, el objetivo final era determinar la fluctuación de velocidad en la boca de la cánula de escape $\widetilde{u}$ como: $\widetilde{u}=\widetilde{u}^{+}+\widetilde{u}^{-}$, por lo que es necesario considerar una relación adicional entre las componentes de la velocidad en el extremo abierto para poder cerrar el problema. En general esta relación puede escribirse simplemente como

$$
\widetilde{u}^{-}(\omega)=R(\omega) \cdot \widetilde{u}^{+}(\omega)
$$

donde $R(\omega)$ es el coeficiente de reflexión complejo en el extremo abierto, cuyo valor será discutido posteriormente. El sustituir la ecuación (2.15) en la ecuación (2.14) conduce a

$$
\widetilde{u}^{+}(\omega)=\frac{\bar{P}(\omega)}{e^{j \beta^{+} L}-R(\omega) \cdot e^{-j \beta^{-L}}}
$$

por tanto, de acuerdo con la expresión (2.15) la fluctuación de velocidad total en el extremo abierto quedaría dada por

$$
\widetilde{u}(\omega)=\widetilde{u}^{+}(\omega)+\widetilde{u}^{-}(\omega)=\widetilde{u}^{+}(\omega) \cdot[1+R(\omega)]
$$


obteniéndose finalmente, a través de la sustitución de la ecuación (2.16) en (2.17), la siguiente representación de la fluctuación de velocidad total

$$
\widetilde{u}(\omega)=\frac{\bar{P}(\omega) \cdot[1+R(\omega)]}{e^{j \beta^{+} L}-R(\omega) \cdot e^{-j \beta^{-L}}}
$$

Retornando al dominio tiempo, aplicando la transformada inversa de Fourier, se obtendrá la expresión de la fluctuación de la velocidad en la boca de salida de la cánula de escape.

Este desarrollo pone de manifiesto que para la obtención de la fluctuación de velocidad $\widetilde{u}(t)$, además de la medida de presión instantánea es necesario disponer de una estimación de la temperatura media del flujo para poder estimar la velocidad media del sonido $c$. Así, también es requerida la medida del flujo másico medio para la determinación de la velocidad media del mismo $U$ y así poder estimar el valor del número de Mach $M$.

\subsubsection{Coeficiente de reflexión en el extremo abierto}

Como se observa en la ecuación (2.18), un paso necesario para la obtención de $\widetilde{u}(\omega)$ es el conocimiento analítico del coeficiente de reflexión o en su defecto su obtención experimental. A este respecto, se ha optado por la utilización de la expresión analítica del coeficiente de reflexión en el extremo abierto corregida con el flujo medio dada por Davies [44]

$$
R(\omega)=R_{M}(\omega) \cdot \exp \left(-j 2 k l_{M}\right)
$$

la cual contiene la influencia de la corrección de longitud en el extremo abierto $l_{M}$, magnitud que proporciona una medida de la acústica del flujo en torno al extremo abierto del conducto. Este efecto está determinado por el flujo local dentro de una región de longitud de escala del orden del radio del conducto.

La dependencia del módulo del coeficiente de reflexión con el número de Mach, $R_{M}(\omega)$, fue estudiada exhaustivamente por Cargill [23] y Munt [125], incluyendo la condición de causalidad y de Kutta completa al borde del conducto. Como comenta Cargill [23], estas dos condiciones son importantes a la hora de determinar el campo sonoro, siendo la causalidad definida como el requerimiento de que el campo sonoro generado por la excitación de un impulso debe anularse antes de que interaccione con la fuente y como consecuencia de ella la fuente se active, mientras que la condición de Kutta se refiere a que la capa vortical debe abandonar el borde de salida del conducto con gradiente de vorticidad cero. 
A su vez Munt [125] derivó una expresión analítica para la teoría lineal de la reflexión de las ondas planas acústicas para el sonido transmitido a través de un extremo abierto de un tubo circular, la cual fue validada experimentalmente por Davies [37]. Ésta predice que en presencia de flujo medio subsónico uniforme y para pequeñas amplitudes acústicas, el coeficiente de reflexión de la presión al final de la cánula es una función del número de Helmholtz, $H e=k a$, y del número de Mach, $M, R_{M}(\omega)=f(k a, M)$. Ambos autores coinciden en que la forma que adopta esta función es la siguiente

$$
R_{M}(\omega)=f(M) \cdot R_{o}(k a)
$$

donde la dependencia frecuencial del coeficiente de reflexión, $R_{o}(k a)$, corresponde al comportamiento del coeficiente de reflexión en ausencia de flujo medio uniforme obtenido por Levine and Schwinger [101], y contiene explícitamente la dependencia con el número de Helmholtz. El factor $f(M)$, por su parte recoge el efecto del flujo, el cual, según Davies [45] adopta una forma algo compleja para el caso de un flujo saliente, forma que no ha sido publicada hasta la fecha.

En la teoría de Munt la condición de Kutta se asume aplicada a los bordes del extremo de la cánula, lo cuál implica la condición de velocidad y presión finita en los bordes. Ante tal suposición, una perturbación acústica del chorro corresponde a una conversión de energía acústica en energía cinética de las perturbaciones vorticales en la capa límite del chorro. De esta forma, Munt [125] encontró que la magnitud del coeficiente de reflexión para todos los números de Mach, si el número de Helmholtz se aproxima a cero es:

$$
\lim _{k a \rightarrow 0}\left|R_{M}(\omega)\right|=1 \quad \forall M
$$

Para números de Helmholtz intermedios y para $M>0$, el coeficiente de reflexión de la presión alcanza un valor máximo superior a 1, valor que Cargill [22] verificó que se obtenía para números de Strouhal, $S_{t}=k a / M$, próximos a $\pi$. Para flujo frío en ausencia de flujo externo en el rango de bajos números de Helmholtz y bajos números de Mach, la forma aproximada y la solución cerrada fue derivada por Cargill [22] [23] distinguiendo entre el caso en que se incluye o no la condición de Kutta (coincidiendo este primer caso con el estudiado por Munt [125]), los cuales serán denotados con los subíndices $K$ y $N_{K}$ respectivamente. Las expresiones obtenidas por Cargill [22], para el caso de $k a \ll 1$ adquieren las formas siguientes

$$
\left|R_{M}(\omega)\right|_{K}=\left|\frac{(1+M) \pi_{o}-1}{(1-M) \pi_{o}+1}\right| \cdot R_{o}(k a)
$$




$$
\left|R_{M}(\omega)\right|_{N K}=\left|\frac{(1+M)}{(1-M)}\right| R_{o}(k a)
$$

donde $\pi_{o}$ representa el polo de inestabilidad para la onda de presión reflejada, cuyo valor en el límite de bajos números de Strouhal es $\pi_{o} \simeq 1 / M$, de manera que el factor que multiplica a $R_{o}(k a)$ es la unidad.

En general se observa un buen acuerdo entre los resultados obtenidos por Cargill y Munt para cualquier rango de número de Strouhal y para números de Mach entre 0 y 0.3 , excepto para el rango de altas frecuencias donde la aproximación $k a \ll 1$ ya no es válida.

En relación con la corrección del extremo abierto con el flujo saliente las observaciones experimentales conducen a la siguiente expresión dada por Davies [44]

$$
l_{M}=l_{o} \cdot\left[1-M^{2}\right]
$$

En la expresión anterior $l_{o}$ representa la corrección de longitud en ausencia de flujo medio. Las modificaciones de la corrección de longitud con el flujo saliente no son muy importantes para números de Mach inferiores a 0.3, aunque algunos autores coinciden en que esta puede expresarse mediante la ecuación (2.23) sin pérdida de validez.

Para la obtención de los valores correspondientes de $R_{o}(k a)$ y $l_{o}$, para flujo medio nulo, pueden emplearse diferentes expresiones dependiendo de las condiciones particulares consideradas asociadas a la forma de la boca de la cánula, es decir, extremo abierto rebordeado o sin reborde exterior. Para el caso práctico será considerado la expresión de $R_{o}(k a)$ para un extremo abierto sin reborde exterior dado por Levine and Schwinger [101] para rangos específicos del número de Helmholtz $k a$. Para el rango de $0<k a<0.2$ el correspondiente coeficiente de reflexión $R_{o}(k a)$ está dado por

$$
R_{o}(k a)=\left[1-\left\{4-f_{o}(k a)\right\} \Lambda\right]^{0.5}
$$

donde $f_{o}(k a)=3.47 \cdot(k a)^{1.9738}$ y $\Lambda=(k a)^{2} /\left(4+(k a)^{2}\right)$.

Para $0.2<k a<1.5$ la expresión obtenida por Davies [45] es.

$R_{o}(k a)=1+0.01336 \cdot(k a)-0.59079 \cdot(k a)^{2}+0.33576 \cdot(k a)^{3}-0.06432 \cdot(k a)^{4}$

Las correcciones de longitud en el extremo abierto en ausencia de flujo medio uniforme son obtenidas a partir de las siguientes expresiones derivadas empíricamente por Davies [40]

$$
l_{o} / a=0.6133-0.1168 \cdot(k a)^{2} \quad k a<0.5
$$




$$
l_{o} / a=0.6393-0.1104 \cdot(k a) \quad 0.5<k a<2
$$

Peters et al. [137] afirman que la medida de la influencia del espesor del muro en las propiedades de reflexión de un extremo abierto confirman la teoría lineal de Ando (1969), que suponía que la corrección de longitud $l_{M}$ de un tubo sin reborde en presencia de flujo medio variaba desde el valor límite a altos números de Strouhal $l_{M} / a=0.61$, el cual es muy próximo al valor dado por Levine \& Schwinger [101] en ausencia de flujo medio, $l_{o} / a=0.6133$, hasta un valor de $l_{M} / a=0.19$ en el límite de bajos números de Strouhal, el cuál es cercano al valor predicho por Rienstra de $l_{M} / a=0.26$.

Incluyendo o no la condición de Kutta en la estimación de la corrección de longitud, Rienstra (1983) encontró un comportamiento no uniforme en la corrección de longitud para $k a \ll 1, M \ll 1$. Para bajas frecuencias en el límite en que se anula el flujo medio $M \rightarrow 0$, con $S_{t}=k a / M \rightarrow \infty$, se encuentra el mismo valor límite que obtuvieron Levine \& Schwinger (1948) [101] en ausencia de flujo medio de

$$
\lim _{M \rightarrow 0, S t \rightarrow \infty} l_{M} / a=0.6133
$$

Este mismo autor encontró que para números de Mach pequeños pero finitos, y para el rango de muy baja frecuencia se verificaba

$$
\lim _{k a \rightarrow 0, S t \rightarrow 0} l_{M} / a=0.2554\left(1-M^{2}\right)^{1 / 2}
$$

Davies [36] ya había puesto de manifiesto el hecho de que la corrección de longitud dependía fuertemente de la frecuencia. Sin embargo los límites de bajos números de Strouhal no fueron alcanzados ya que los datos considerados por Davies (1980) [36] y Munt (1990) [125] fueron obtenidos para $k a>0.1$ y $M<0.3$, los cuales sí que son válidos en el caso que nos ocupa (el escape en motor). Por otro lado los experimentos llevados a cabo por Peters et al. (1993) [137], permitieron observar que para altos números de Strouhal el valor límite encontrado para la corrección de longitud es independiente del número de Mach, mientras que para bajos números de Strouhal se obtuvo el valor límite de $l_{M} / a=0.19$, quedando sin verificar experimentalmente la dependencia con el número de Mach predicha por Rienstra (1983), recogida en la ecuación (2.28).

\subsubsection{Validación numérica}

Con el fin de obtener una primera estimación de la fiabilidad del procedimiento propuesto para la estimación de la fluctuación de la velocidad en 
la boca de la cánula de escape, y debido a que no se dispone de valores experimentales de dicha magnitud, por los problemas comentados anteriormente (sección 2.2), se realiza un experimento numérico basado en el trabajo de Payri et al. [132]. Mediante, este cálculo numérico se simulan las medidas de presión reales y se calcula la fluctuación de velocidad en la boca del escape $(\widetilde{u})$. El valor calculado de $\widetilde{u}$ servirá de referencia para la comparación con la misma fluctuación estimada, como se indicó en la sección anterior, a partir de las presiones simuladas en la cánula. Siendo posible, de este modo, determinar la validez de las diferentes hipótesis consideradas en el desarrollo teórico y obtener una primera valoración de la sensibilidad del procedimiento.

Como resultado adicional, en esta sección, se obtiene una primera indicación de los problemas que pueden esperarse en el experimento real, posponiendo el estudio de la influencia de las incertidumbres en las magnitudes experimentales relevantes sobre los resultados del método hasta la sección siguiente.

La configuración escogida en la simulación numérica, es un motor de gasolina de 4 cilindros, con un silenciador trasero consistente en un volumen vacío de 20 litros, seguido de una cánula de escape de $0.25 \mathrm{~m}$ de longitud y $45 \mathrm{~mm}$ de diámetro. La presión se calcula en un punto situado a $0.1 \mathrm{~m}$ del extremo abierto, de esta forma la posición de medida puede considerarse realista.

Se consideran tres regímenes de giro diferentes del motor cubriendo el rango normal de funcionamiento de este tipo de motores: $1000 \mathrm{rpm}, 3000 \mathrm{rpm}$ y $6000 \mathrm{rpm}$, esperando que aparezcan rasgos diferentes a cada régimen, debido principalmente a la diferente importancia relativa de las fluctuaciones y valor medio de velocidad, que nos permita hacer un estudio global de todos los posibles limites de precisión que pueden presentarse a la hora de aplicar este procedimiento de estimación. Efectivamente, se comprobará posteriormente cómo la posible presencia de reflujos a bajos regímenes de giro puede dificultar el procesado de los datos.

En primer lugar, en la figura 2.1 se muestran las señales de presión calculadas a $0.1 \mathrm{~m}$ del extremo abierto. El rasgo más importante de estos gráficos es la posibilidad de estimar la amplitud esperada de las señales medidas. Puede observarse como mientras a $1000 \mathrm{rpm}$ las amplitudes de pico a pico se encuentran sobre $3 \mathrm{kPa}$, a $3000 \mathrm{rpm}$ esta amplitud se reduce a 0.7 $k P a$ llegando a ser de $0.1 \mathrm{kPa}$ cuando se considera el régimen de $6000 \mathrm{rpm}$. $\mathrm{El}$ hecho de que esta oscilación disminuya al aumentar la velocidad de giro del motor podría afectar al registro de las medidas, en el sentido de que la precisión puede ser mayor a bajos regímenes de giro, en el cual la fluctuación 


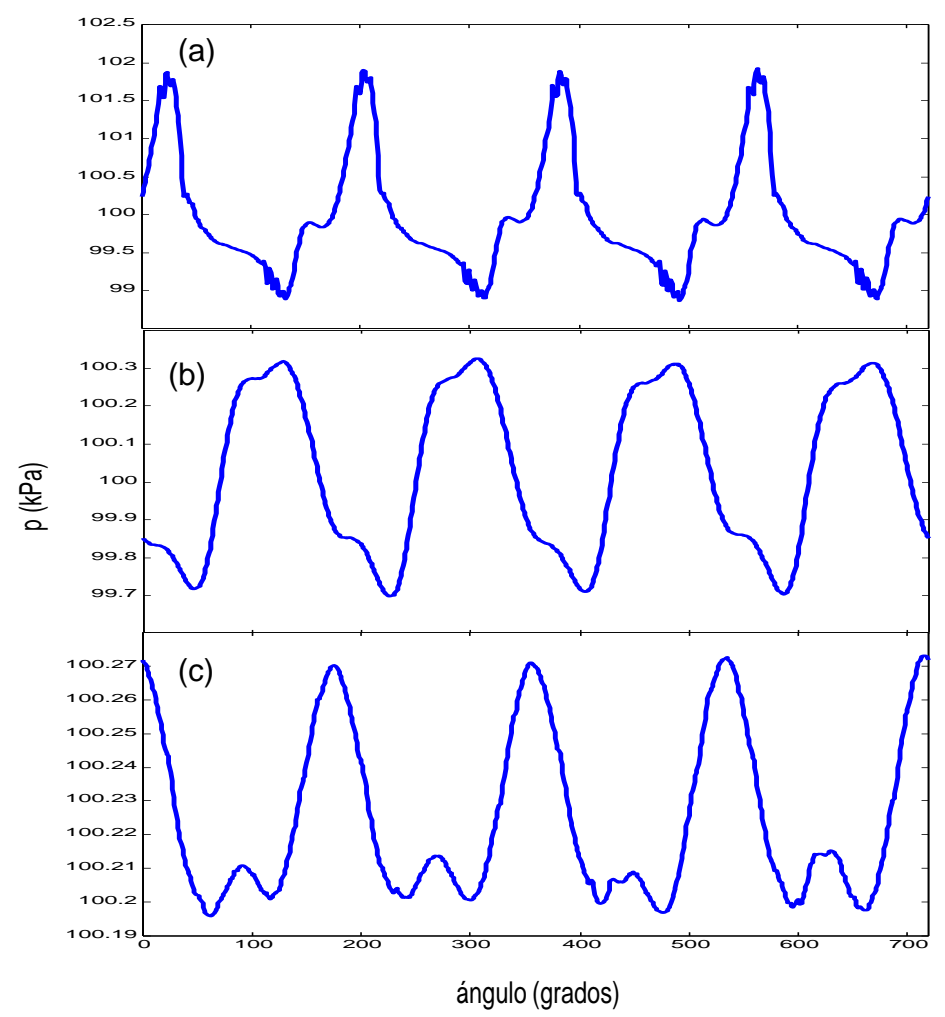

Figura 2.1: Presión calculada a 1000 rpm (a), 3000 rpm (b) y 6000 rpm (c).

periódica inducida por la descarga de los cilindros del motor prevalezca sobre cualquier fluctuación local turbulenta. Una oscilación semejante no puede ser considerada en la simulación numérica llevada a cabo, pero sí que podría estar presente en los experimentos reales, ver figura 2.12, siendo ésta más importante a altos regímenes de giro, como comentan Kunz y García [92], pudiéndose, incluso, presentar la situación de que la fluctuación de presión sea del mismo orden que el umbral del transductor de presión, siendo por tanto imposible discernir entre la señal y el ruido, ya que estaría en el límite de la relación señal-ruido de dicho transductor. Éste podría considerarse como un primer problema potencial en la aplicación de esta técnica.

Una vez descritas las señales, aplicaremos la teoría presentada en la sección anterior a los registros de presión calculados analizando los resultados derivados. En la figura 2.2 (a) se muestran los resultados obtenidos en el domino del tiempo para un régimen de giro de $1000 \mathrm{rpm}$, en particular se 
presenta la comparación directa entre la fluctuación del caudal volumétrico en la boca de salida de la cánula calculada directamente y la obtenida a partir del cálculo de la presión registrada en la cánula. En ella puede observarse que, aunque se aprecien algunas diferencias en la forma de las curvas la tendencia general es reproducida. Debe recalcarse, en referencia a este último comentario, el hecho de que estas diferencias aparecen precisamente en los intervalos de tiempo correspondientes al reflujo en el conducto, aspecto ya citado anteriormente como posible influencia. Para verificar si estas diferencias se deben exclusivamente a la presencia de reflujo a este régimen del motor y no, por ejemplo, al hecho de considerar un temperatura media, se obtiene la estimación de la fluctuación del caudal volumétrico, representado en la figura 2.2 (b), considerando las modificaciones que el reflujo introduce en el coeficiente de reflexión de acuerdo con la expresión derivada por Davies [39]

$$
R_{M}(\omega)=R_{o}(k a) \cdot\left[\left(1-\alpha_{s} M\right) /\left(1+\alpha_{s} M\right)\right]^{0.9}
$$

para el rango de $0<\alpha_{s} M<0.6$, ó $0<M<0.4$. Donde el factor $\alpha_{s}$ representa el factor de "restricción acústica del flujo" y su valor depende de la geometría del extremo. En nuestro caso, se escogió $\alpha_{s}=1.47$ en acuerdo con las consideraciones establecidas por Davies para un extremo abierto emplazado en una pared y alineado con ella.

El buen acuerdo observado entre ambos resultados en los instantes correspondientes al reflujo y a la salida de flujo (levemente sobrestimados en este caso), implica que para bajos regímenes de giro el reflujo juega un papel importante, al menos a bajas frecuencias, como ratifican las figuras 2.3 y 2.4 , donde está representado el espectro calculado para estas fluctuaciones de caudal volumétrico en una escala de dB arbitraria, en la parte superior, y la fase, en la parte inferior, teniendo en cuenta las mismas consideraciones que para las gráficas de la figura 2.2. Por claridad, en la fase sólamente se han considerado los armónicos correspondientes a las frecuencias de encendido del motor.

Sin embargo, estas diferencias no afectan de forma importante al espectro obtenido, en particular se puede ver cómo únicamente los armónicos del motor $\mathrm{H} 4$ y $\mathrm{H} 8$ son sobrestimados unos 4 ó $5 \mathrm{~dB}$ y algunas diferencias adicionales entre ambos espectros aparecen a baja frecuencia donde los niveles espectrales estimados cambian su comportamiento de una sobrestimación a una subestimación para el nivel espectral a $8 \mathrm{~Hz}$, y una correcta reproducción a 17 y $25 \mathrm{~Hz}$ para esta segunda estimación (ver figura 2.3), donde las condiciones de reflujo se incluyen en el coeficiente de reflexión considerado.

Si se realiza un análisis similar con el ángulo de fase de estos caudales volumétricos, considerando sus valores únicamente para los armónicos princi- 


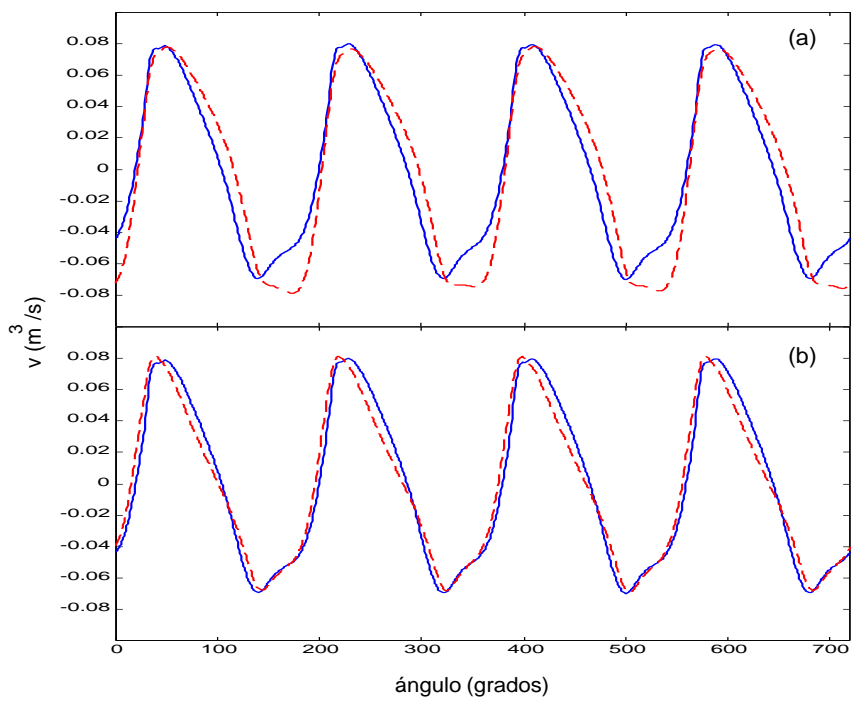

Figura 2.2: Comparación del caudal volumétrico calculado (linea continua) y estimado (linea discontinua) en el extremo abierto a $1000 \mathrm{rpm}$ (a) sin considerar el reflujo y (b) considerando los efectos del reflujo.

pales, por claridad, se obtienen los mismos resultados en ambos casos, tal y como se muestra en la parte inferior de las figuras 2.3 y 2.4 .

La situación cambia al aumentar el régimen de giro, como se observa en las figuras 2.5 y 2.6, donde se muestran los resultados obtenidos a $3000 \mathrm{rpm}$. Ahora la coincidencia de las curvas obtenidas en el dominio del tiempo, figura 2.5, es excelente, y lo mismo puede decirse para los armónicos principales tanto en el espectro de la amplitud del caudal volumétrico como para el ángulo de fase del mismo, ver figura 2.6.

Si nos trasladamos hacia regímenes más elevados como 6000 rpm, cuyos resultados se muestran en las figuras 2.7, y 2.8, la situación es similar, excepto que ahora la reproducción de las curvas en el dominio del tiempo no es tan perfecta como en los casos anteriores. Sin embargo, como puede apreciarse en el espectro y ángulo de fase del caudal volumétrico (figura 2.8), las pequeñas diferencias parecen estar localizadas a frecuencias medias, lo cual no implica una influencia significativa en la forma general de la fluctuación. De modo que las diferencias que aparecen para frecuencias comprendidas entre los armónicos H6 y H8 son las responsables de las discrepancias apreciadas en las curvas en dominio tiempo. Aún así los resultados obtenidos para los armónicos principales siguen siendo excelentes. 


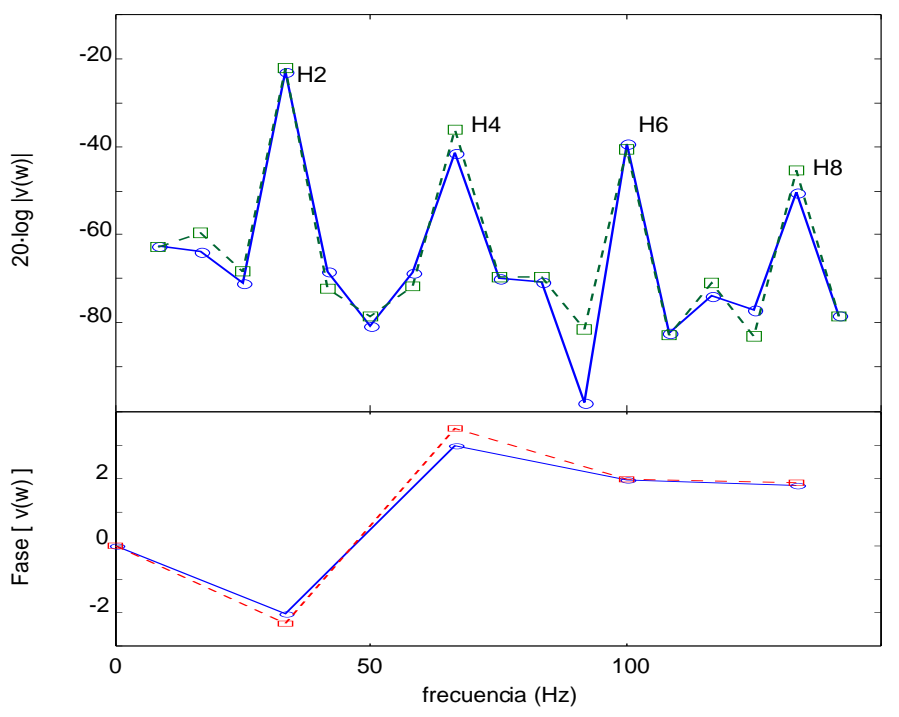

Figura 2.3: Comparación del gasto volumétrico calculado ( $\bigcirc)$ y estimado ( $\square$ ) en el extremo abierto a 1000 rpm, considerando reflujo: amplitud (arriba) y fase (abajo). Frecuencia de los armónicos de motor $\mathrm{Hm}=m \times 1000 / 60(\mathrm{H2}=33.3 \mathrm{~Hz}$, etc.

A partir del análisis de los resultados anteriores, se puede inferir que para una velocidad de giro del motor intermedia, como es por ejemplo 3000 rpm, las condiciones son tales que las principales hipótesis consideradas en el desarrollo teórico son válidas. En particular, la propagación lineal a la velocidad del sonido correspondiente a una temperatura media, y el coeficiente de reflexión en el extremo abierto dado por la ecuación (2.19). Con respecto a la primera hipótesis (temperatura media) los resultados mostrados en la figura 2.9 son concluyentes. En ella se presenta la velocidad del flujo así como su temperatura en el punto de medida de la presión en la cánula de salida para los tres regímenes de giro considerados. En los resultados a $1000 \mathrm{rpm}$ representados en la figura 2.9(a), es evidente que la llegada del reflujo desde el extremo abierto al punto de medida (velocidades de flujo negativas) provoca un aumento y un cambio acusado en la temperatura. Por ello, en este caso el concepto de temperatura media es en si mismo problemático, junto con el hecho de que estas discontinuidades en la periodicidad de la temperatura afectan a la propagación de las ondas. De aquí se desprenden las explicaciones dadas a los resultados presentados en la figura 2.2, caudal calculado a 1000 rpm sin y con correcciones con el reflujo; ya que, a pesar de que en el primer caso se han tenido en cuenta la fase principal de las fluctuaciones del flujo a 
2.3. Estimación de la fluctuación de velocidad en la boca de la cánula de escape33

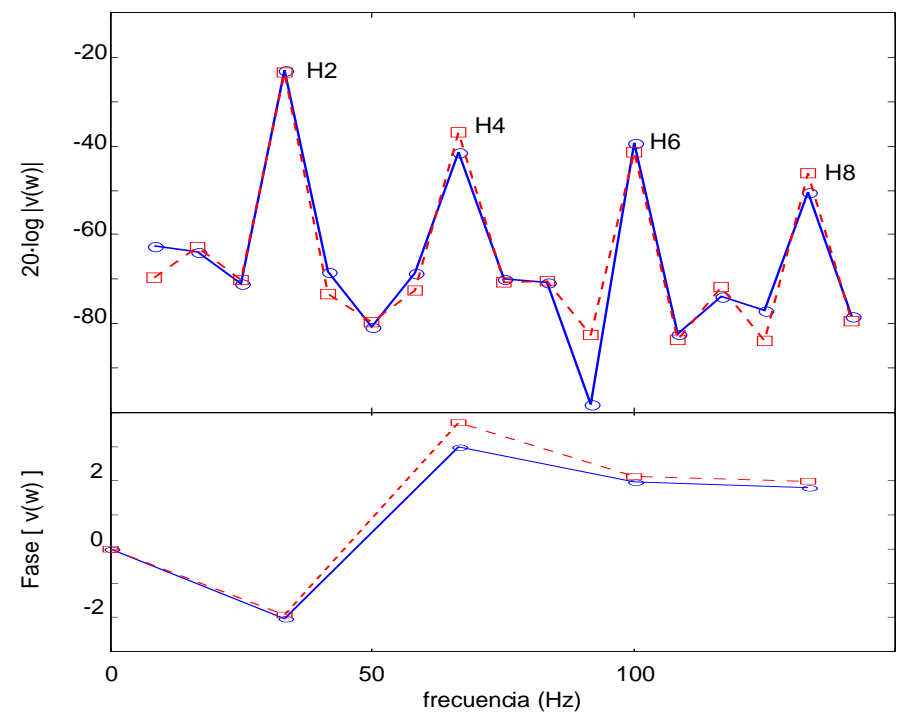

Figura 2.4: Comparación del gasto volumétrico calculado (○) y estimado ( $\square$ ) en el extremo abierto a 1000 rpm, sin considerar reflujo: amplitud (arriba) y fase (abajo). Frecuencia de los armónicos de motor $\mathrm{Hm}=m \times 1000 / 60(\mathrm{H} 2=33.3 \mathrm{~Hz}$, etc.

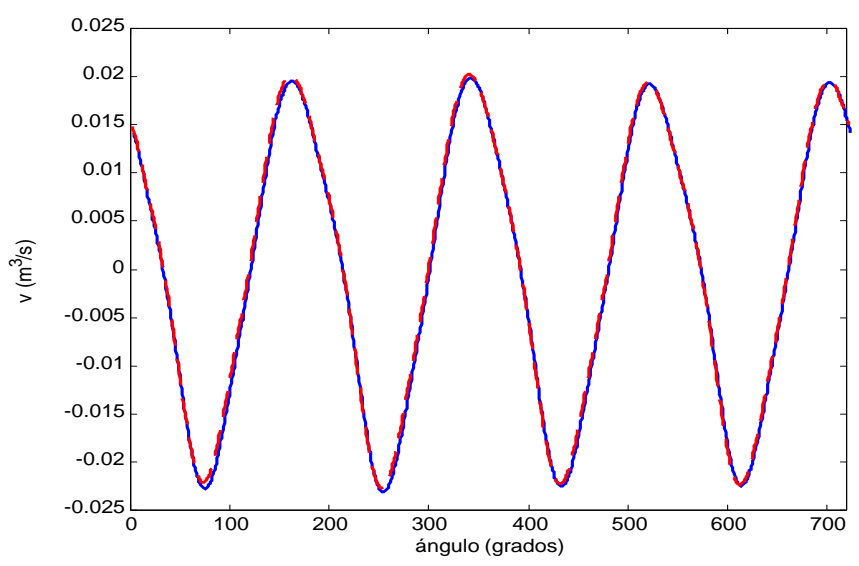

Figura 2.5: Comparación del gasto volumétrico en el extremo abierto a 3000 rpm: calculado (linea continua) y estimado (linea discontinua 


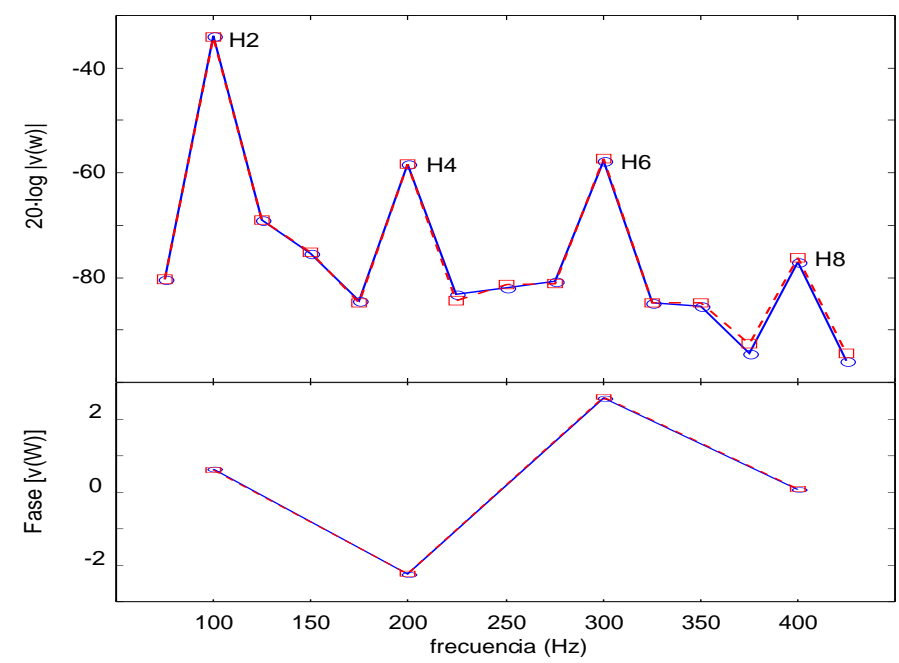

Figura 2.6: Comparación del gasto volumétrico calculado (○) y estimado ( $\square$ ) en el extremo abierto a $3000 \mathrm{rpm}$, sin considerar reflujo: amplitud (arriba) y fase (abajo). Frecuencia de los armónicos de motor $\mathrm{Hm}=m \times 3000 / 60(\mathrm{H} 2=100 \mathrm{~Hz}$, etc.

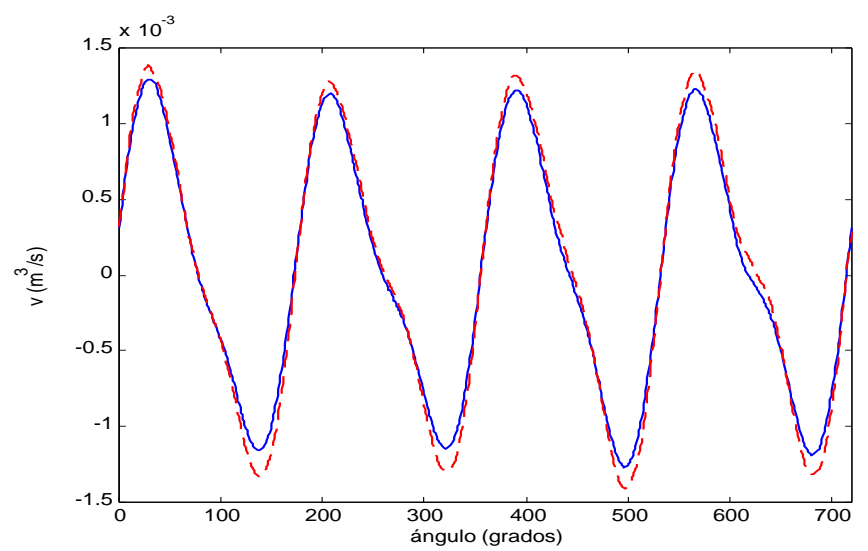

Figura 2.7: Comparación del gasto volumétrico en el extremo abierto a 6000 rpm: calculado (linea continua) y estimado (linea discontinua). 


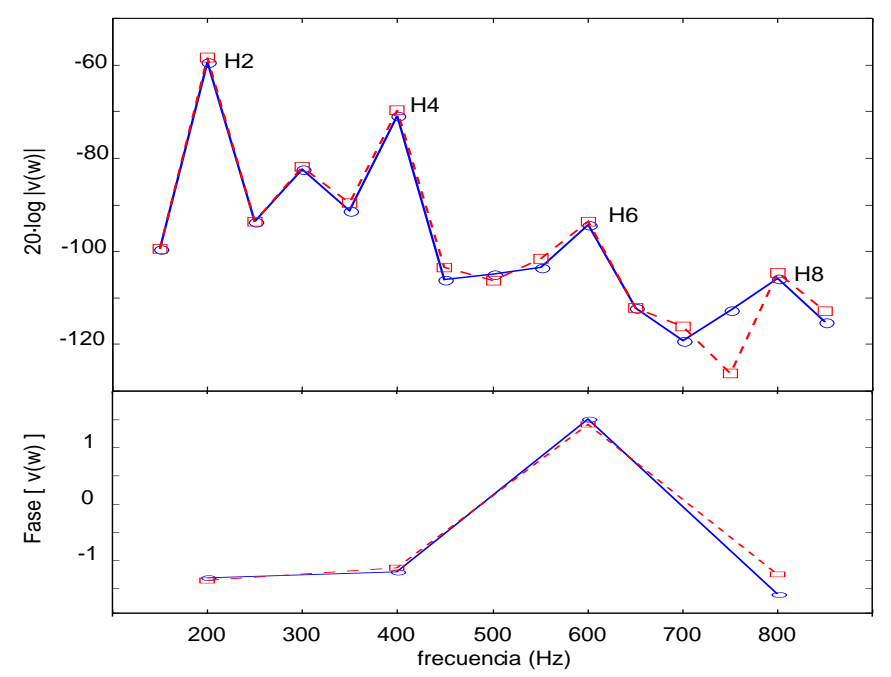

Figura 2.8: Comparación del gasto volumétrico calculado (○) y estimado ( $\square$ ) en el extremo abierto a 6000 rpm, sin considerar reflujo: amplitud (arriba) y fase (abajo). Frecuencia de los armónicos de motor $\mathrm{Hm}=m \times 6000 / 60$ (H2=200 Hz, etc.)

través de la velocidad media del sonido y se han introducido los detalles de la propagación de onda por medio de vectores de ondas complejos, que contienen contribuciones tales como la de los efectos de atenuación viscosa, los efectos del reflujo en el coeficiente de reflexión en el extremo abierto se han perdido.

Por el contrario cuando se consideran regímenes de giro superiores (3000, $6000 \mathrm{rpm}$ ), la velocidad es siempre positiva, así que el coeficiente de reflexión no debe incluir ningún término asociado al reflujo. Además, las fluctuaciones de temperatura son pequeñas, lo cual permite validar la consideración de velocidad del sonido media como hipótesis aproximada del comportamiento real. Esto justifica el hecho de que los resultados obtenidos a 3000 y $6000 \mathrm{rpm}$ sean, en este sentido, mejores que los obtenidos a $1000 \mathrm{rpm}$.

Con respecto a la desviación a muy altos regímenes de giro del motor, la fuente de error más probable podría encontrarse en referencia a las hipótesis de reflexión consideradas, ya que por un lado, las correcciones asintóticas con el flujo utilizadas para el caso límite de pequeños números de Mach y Strouhal en el seno de un flujo frío, podría no ser adecuada para el caso de altos regímenes de giro donde la temperatura de los gases de escape es más elevada. Ya que, se espera que la gran diferencia entre la temperatura del flujo y el aire que rodea a la salida de la cánula altere los fenómenos de reflexión y transmisión, como constatan Alfredson y Davies [2]. Por otro 


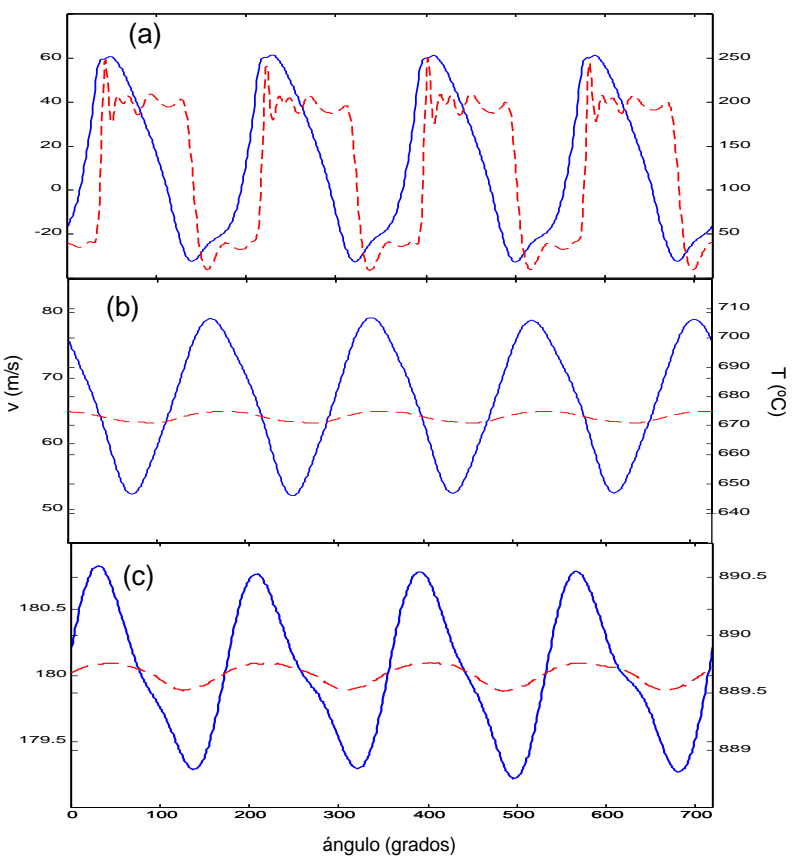

Figura 2.9: Velocidad (linea continua, eje izquierdo) y temperatura (linea discontinua, eje derecho) calculadas a $1000 \mathrm{rpm}$ (a), $3000 \mathrm{rpm}$ (b) y $6000 \mathrm{rpm}$ (c).

lado, es interesante notar el cambio producido en la importancia relativa del valor medio y de la fluctuación del gasto, en definitiva, de la velocidad de fluctuación, cuando aumenta la velocidad de giro del motor. Esto podría definir también una limitación en el método relacionada con la correlación con el ruido de escape, ya que obviamente las contribuciones del ruido de flujo sobre el ruido de escape total, a altos regímenes, podrían no estar completamente representadas en la estructura de la técnica propuesta, lo cual introduce una dificultad adicional.

Finalmente, para evaluar las limitaciones potenciales del método en el cálculo del ruido de escape radiado, se examina la derivada temporal de la fluctuación del caudal volumétrico en el extremo abierto, ya que es ésta la magnitud que está directamente relacionada con el espectro de ruido radiado [136]. El buen acuerdo mostrado para la estimación de la fluctuación del caudal volumétrico a $3000 \mathrm{rpm}$, figura 2.5, se obtiene de nuevo para la derivada temporal de esta magnitud como se observa en la figura 2.10(a). Este buen acuerdo entre el cálculo y la estimación de la derivada temporal se extiende al cálculo de su nivel espectral, tal y como muestra la figura 2.10(b). 

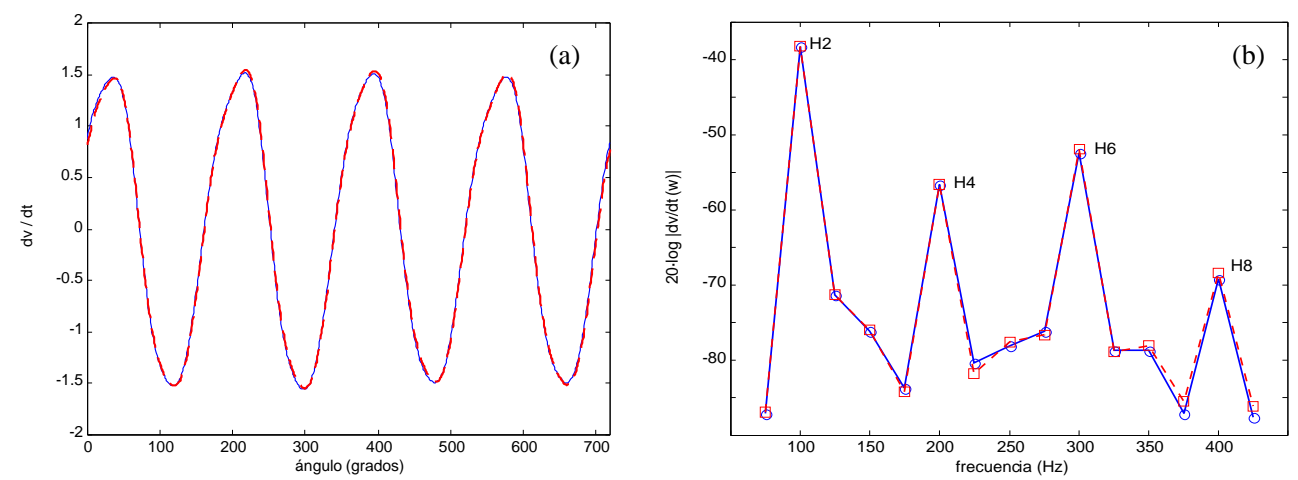

Figura 2.10: Comparación entre la derivada temporal del gasto volumétrico en el extremo abierto de la cánula de escape a 3000 rpm: (a) amplitud calculada(linea continua) y estimada (linea discontinua) y (b) espectro calculado ( $\bigcirc)$ y estimado $(\square)$.

De esta discusión se derivan las siguientes conclusiones a tener en cuenta en la evaluación de la situación experimental:

1. La robustez del procedimiento es razonablemente alta, ya que las incertidumbres máximas en la estimación del caudal volumétrico en la boca de salida de la cánula de escape debidas a las hipótesis consideradas están alrededor del $\pm 3 \%$, lo cual constituye un valor de error de estimación muy aceptable.

2. Dadas las bajas amplitudes que pueden detectarse y la presencia, esperable, de ruido en las medidas reales, ha de ponerse un cuidado especial en las medidas de presión en cánula.

3. Ha de tenerse en cuenta el potencial aumento del error debido al empleo de una velocidad de propagación media cuando existe presencia de reflujos y a la no inclusión, en el modelo, de las correspondientes correcciones con el reflujo en el coeficiente de reflexión.

4. Finalmente, se debe prestar atención a la dependencia de los valores relativos de velocidades medias y fluctuantes con el régimen de giro del motor, que podrían limitar las posibilidades de correlacionar los resultados obtenidos con las medidas de ruido de escape. 


\subsubsection{Discusión sobre el error cometido en la estimación del caudal volumétrico}

Para cuantificar la precisión del método propuesto, esto es, definir el error cometido en la estimación del espectro del caudal volumétrico, se deben considerar las incertidumbres asociadas al método de estimación presentado en la sección 2.3. En primer lugar, se evalúa el error introducido por las hipótesis empleadas en el método de estimación, a través de un análisis cuantitativo de los resultados mostrados en la sección anterior. Posteriormente, son analizadas todas las posibles fuentes de error procedentes de las incertidumbres en la determinación de las diferentes magnitudes consideradas en el método de estimación como: temperatura, gasto másico, posición del transductor, etc. La influencia del error de estos datos experimentales puede analizarse de diversas formas, en este caso, y en la línea de la sección anterior, se escoge, nuevamente, la simulación numérica para su determinación, procedimiento que está avalado por trabajos como los de Bodén et al. [18].

Para cualquier contribución considerada, la desviación del valor predicho frente al valor real, error absoluto, es calculado como el error cuadrático medio (RMSE) [13] definido como

$$
R M S E=\sqrt{\frac{1}{N_{t}} \cdot \sum_{t=1}^{N_{t}}[\widehat{\dot{v}}(t)-\dot{v}(t)]^{2}}
$$

donde $\widehat{\hat{v}}(t)$ representa la estimación de la derivada temporal de la fluctuación del caudal volumétrico y $\dot{v}(t)$ es el cálculo teórico de esta magnitud. Sin embargo, para obtener el error global estándar a partir de todas las contribuciones mencionadas, de cara a su posterior combinación, es conveniente considerar el error relativo $(R E)$ de estas magnitudes (Barford [8]), que se obtiene a través de la siguiente expresión

$$
R E=\sqrt{\sum_{t=1}^{N_{t}}[\widehat{\dot{v}}(t)-\dot{v}(t)]^{2} / \sum_{t=1}^{N_{t}}[\dot{v}(t)]^{2}}
$$

Como ya se mencionó, la magnitud que está directamente relacionada con el espectro de ruido exterior es la derivada temporal del caudal volumétrico, como defienden Landau [95] y Morse [121], por lo que el error debido a las hipótesis empleadas será evaluado a través de esta magnitud. De manera que aplicando la ecuación (2.31) a los resultados mostrados en la sección 2.3.2, se obtiene que el error relativo en el cálculo de la derivada temporal del caudal volumétrico en el extremo abierto es en promedio de un $\pm 3 \%$. 
Una vez evaluado el error debido a las hipótesis empleadas en el procedimiento de estimación de la fluctuación del caudal volumétrico en el extremo abierto, se pasa a estimar el error asociado a las incertidumbres de los datos experimentales. Para ello, se debe escoger el valor de dicha magnitud (fluctuación del caudal volumétrico) a un régimen de giro tal que se minimicen las desviaciones debidas a las hipótesis consideradas en el método de estimación, o lo que es lo mismo el régimen al que las hipótesis resulten más adecuadas. Éste, como se vio en la sección 2.3 .2 es $3000 \mathrm{rpm}$. De esta forma, es posible estudiar únicamente la influencia de las incertidumbres de las variables de medida independientemente del error asociado a las hipótesis.

La precisión en el método de estimación, se determina a través de la comparación directa entre las estimaciones realizadas para los valores extremos de cada magnitud medida (dentro de su rango de precisión), y su valor correcto calculado, disponible del proceso de simulación numérica.

En primer lugar, se investiga la influencia de las incertidumbres en la determinación de la temperatura media del flujo en el punto de medida, ya que esta estimación fijará la velocidad del sonido asumida. Además, para un gasto másico dado, suponiendo que éste está determinado con suficiente precisión, cualquier error en la determinación del valor medio del número de Mach estaría asociado al error en la estimación de la temperatura (y por tanto de la densidad). Para analizar cuáles son los factores que podrían estar influenciando la medida de temperatura, es necesario considerar aquéllos relacionados con el proceso de medida en sí mismo, así como los relacionados con el procesado de la señal. De acuerdo con esto, se considera que el error en la determinación de la temperatura a partir de la medida con un termopar es menor a $\pm 2 \mathrm{~K}$, el cual comprende los dos errores cometidos en su determinación experimental, uno debido a las medida de voltaje y el otro debido al sistema de adquisición. A partir de la ecuación (2.31) el error relativo en $\dot{v}(t)$ asociado con esta incertidumbre en la medida de la temperatura es $\pm 3 \%$.

La incertidumbre en la determinación de la temperatura no parece conllevar importantes incertidumbres en la estimación del caudal volumétrico en el extremo abierto. A fin de mostrar claramente esta influencia, se ha escogido la representación de este efecto para un caso en que la diferencia de temperatura no se restrinja únicamente al límite experimental $( \pm 2 \mathrm{~K})$, sino que sea más acusada ( $\pm 50 \mathrm{~K})$. De este modo, en las figuras $2.11(\mathrm{a})$ y $2.11(\mathrm{~b})$ se muestran los resultados obtenidos a $3000 \mathrm{rpm}$, donde se representan tres curvas: una correspondiente a la temperatura media empleada en el cálculo, y las otras dos curvas correspondientes respectivamente a una subestimación y una sobrestimación de 50 grados en la temperatura media. La influencia de la 
temperatura en las curvas en el dominio temporal es evidente, figura 2.11(a); sin embargo, no parece haberse perdido información relevante, como confirma su correspondiente espectro, figura 2.11(b), donde las máximas diferencias apreciadas en los armónicos principales se encuentran en torno a $1 \mathrm{~dB}$, lo cual constituye el error experimental usual.
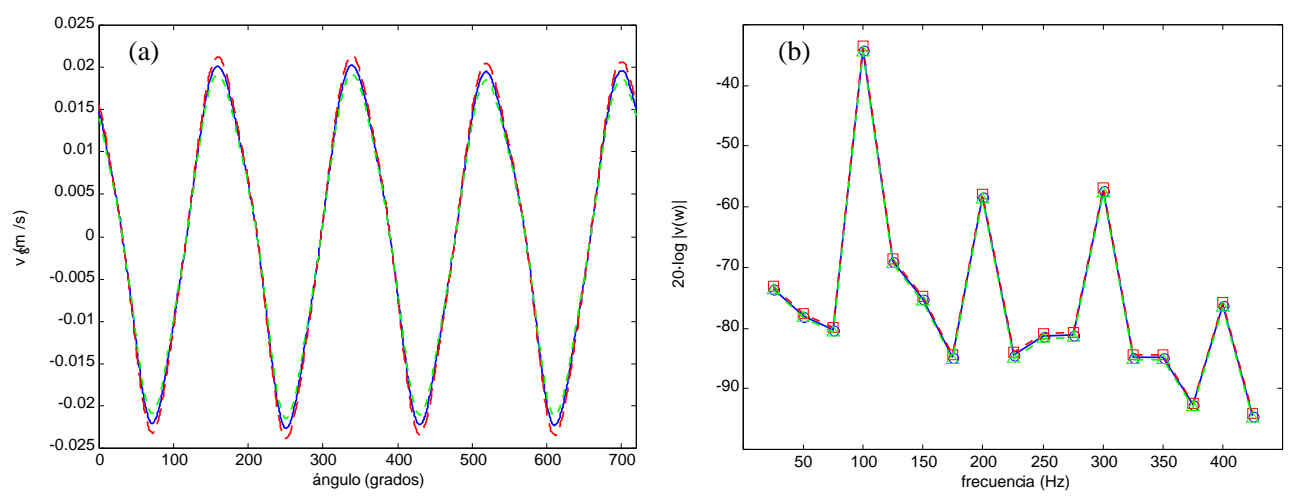

Figura 2.11: Influencia de la estimación de la tempertura en el gasto volumétrico a 3000 rpm: (a) amplitud (linea continua (temperatura T), linea discontinua (temperatura $T+50)$, linea punteada (temperatura T-50)) y (b) espectro ((○) a temperatura $T$, ( $\square)$ a temperatura $T+50$ y ( $\triangle$ ) a temperatura $T-50)$.

Como se ha mencionado en el párrafo anterior, el otro factor importante en la determinación del número de Mach es el error asociado a la determinación del gasto másico, el cual se asume en torno a $\pm 0.01 \mathrm{~kg} / \mathrm{s}$ de acuerdo con su proceso de medida. Este error es propagado a la determinación de $\dot{v}(t)$ produciendo una incertidumbre en su magnitud de $\pm 1.4 \%$.

El método de estimación descrito en este trabajo se basa en el conocimiento de la medida de presión en un punto dado de la cánula. Consecuentemente, los errores asociados con la determinación de este punto, esto es, con la precisión de la medida de la posición del transductor deben tenerse en cuenta. En relación a esta incertidumbre en la posición del transductor considerada en torno a $\pm 1 \mathrm{~mm}$ el error relativo resultante es de $\pm 2 \%$.

Finalmente, con el objeto de considerar todos los posibles errores en la estimación de la derivada temporal del caudal volumétrico, se analiza el error debido a las incertidumbres en la frecuencia, las cuales pueden influir en la determinación de esta magnitud a través de las transformaciones, de dominio tiempo a dominio frecuencia y viceversa, llevadas a cabo en el procedimiento propuesto. Como es sabido la frecuencia viene determinada por el régimen 
de giro del motor $(n)$, siendo directamente proporcional a él, por lo que cualquier imprecisión en la medida del régimen de giro se transformará en una incertidumbre en la estimación de la frecuencia. Se adopta una incertidumbre de $\pm 5 \mathrm{rpm}$ como valor típico de la imprecisión observada en la medida del régimen de giro, lo cual conlleva un error de $\pm 3 \%$ en la estimación de la derivada temporal del caudal volumétrico en la boca de la cánula de salida.

Combinando todos los errores descritos anteriormente de acuerdo con la ecuación (2.31), es posible obtener el máximo error asociado a la estimación de la derivada temporal del caudal volumétrico en el extremo abierto como $\pm 5 \%$. Desde el punto de vista de la predicción del ruido de escape a través de esta estimación, esta incertidumbre implicaría un error de $\pm 0.7 \mathrm{~dB}$. Este hecho confirma que no existe pérdida relevante de información en la estimación, quedando este error dentro del error experimental habitual que se encuentra en torno a $\pm 1 \mathrm{~dB}$ (ver apéndice $\mathrm{C}$ ).

\subsection{Validación del método con líneas de escape rea- les: resultados y discusión}

Con el objeto de evaluar la potencia del método, se ha estimado la fluctuación del caudal volumétrico en la sección de salida de la cánula de escape empleando las medidas de presión realizadas en esta misma cánula. Los experimentos se llevaron a cabo en el escape del mismo motor considerado en la simulación numérica descrita en la sección 2.3.2 (motor de gasolina de cuatro cilindros de 2 litros con aspiración natural), salvo que en este caso se adopta una línea de escape realista. El motor está montado en un banco de pruebas regulado con un freno eléctrico de corrientes de Foucault. El registro de presión se realiza en tres puntos de la cánula de escape situados a: 0.1, 0.15 y $0.2 \mathrm{~m}$ de la boca de salida de la misma, mediante transductores piezoeléctricos del tipo KISTLER 7031, cuyas características técnicas son tratadas en el apéndice C.

La posición de los transductores fue escogida con el criterio de garantizar que el flujo sea esencialmente unidimensional en el punto de medida y que cualquier perturbación originada por las singularidades del escape (curvaturas del conducto, etc.) no estén afectando a los registros de presión realizados. Dentro de esta región del conducto, Davies [40] considera que el campo sonoro puede describirse satisfactoriamente como una onda plana.

Al igual que en el caso de la simulación numérica, se cubre un rango normal de funcionamiento para este tipo de motores: régimen de giro de 1000 
rpm a $6000 \mathrm{rpm}$, con pasos de $250 \mathrm{rpm}$, a fin de obtener una elevada información sobre los cambios en el comportamiento de las componentes de presión al aumentar el régimen de giro del motor. La adquisición de las ondas de presión está sincronizada con el ángulo de giro del cigüeñal, registrándose 10 ciclos del motor con la finalidad de verificar la estacionariedad y periodicidad de los fenómenos, y disponer de una mejor resolución frecuencial, parámetro fundamental en el caso que nos ocupa.

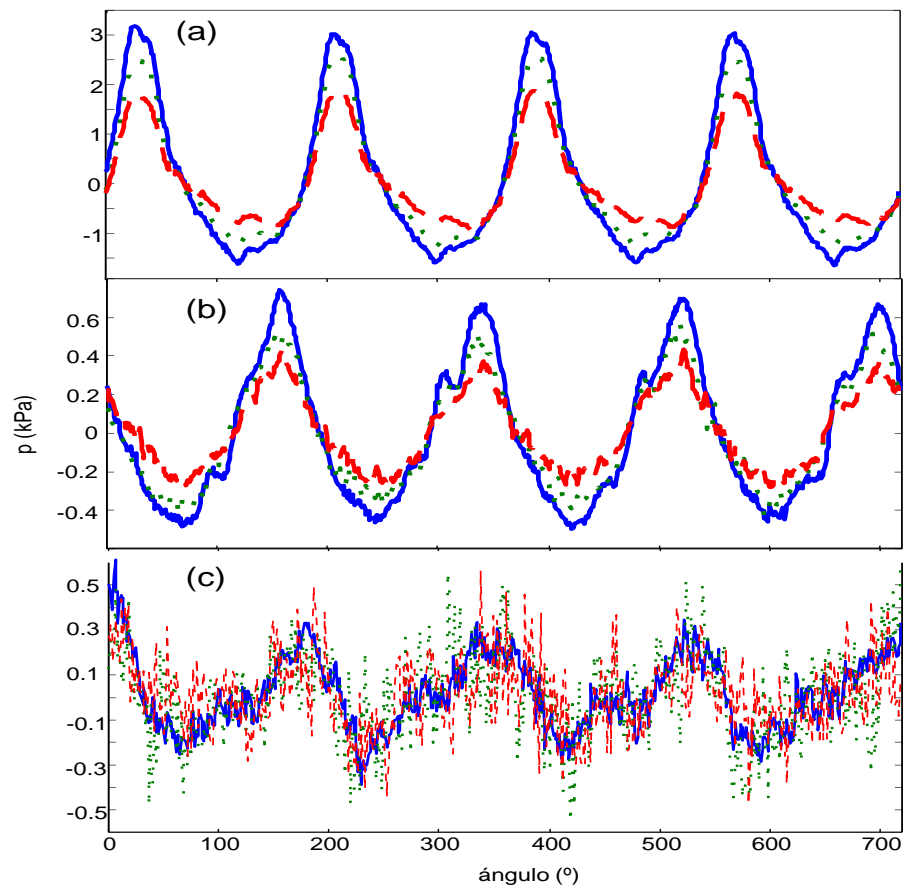

Figura 2.12: Medidas de presión en conducto a 1500 (a), 3000 (b) y 6000 rpm (c), en los puntos localizados a $0.2 \mathrm{~m}$ (linea solida), $0.15 \mathrm{~m}$ (linea punteada) y $0.1 \mathrm{~m}$ (linea discontinua) del extremo abierto.

Debe comentarse que es importante calibrar los transductores de presión empleados en la medida de presión en cánula antes de realizar cualquier ensayo, ya que ellos fijan la medida en la que se basa todo el procedimiento descrito; este proceso de calibración y los equipos empleados para ello serán descritos en el apéndice $\mathrm{C}$ de la presente tesis doctoral. Sin embargo, debe añadirse que estas medidas pueden estar afectadas por varias incertidumbres, como el ruido debido a la propia vibración del sistema de escape [131] y las limitaciones técnicas que poseen dichos transductores (abordados en el apéndice mencionado). 
Por otro lado, la medida de temperatura de los gases en la cánula de escape se realiza en la boca de salida de la cánula, valor que puede admitirse como válido aunque ésta no sea exactamente la medida más adecuada para obtener la temperatura media del flujo en el tramo de propagación de la onda desde el punto de medida de presión a la sección de salida, ya que depende del perfil axial [41] de la distribución de temperatura de los gases de escape en la cánula, evaluación que constituye una tarea bastante compleja por la dependencia de estos gradientes con el flujo de calor convectivo en las paredes, cuya temperatura es función de las condiciones ambientales fuera de la cánula. Hay que recordar que los valores de las propiedades físicas del gas como viscosidad y conductividad térmica aumentan significativamente con la temperatura mientras que $\gamma$ decrece, modificando este efecto el valor de la velocidad del sonido; sin embargo, se comprobó en la sección anterior que la incertidumbre en la temperatura conlleva un error de aproximadamente un $\pm 3 \%$ en la estimación del caudal volumétrico, y por tanto no parece ser un factor determinante del procedimiento presentado.

Los registros de presión realizados en un sistema de escape complejo a 1000, 3000 y 6000 rpm en los tres puntos de medida considerados se representan en la figura 2.12, proporcionando una primera idea de la complejidad real de las medidas, lo cual permitirá comprobar la validez de las conclusiones derivadas de la aplicación del procedimiento descrito sobre dichas medidas. En esta figura se observa como a 1000 rpm los gráficos de presión están bastante libres de ruido, figura 2.12(a), por lo que la tendencia esperada de decaimiento de la amplitud de la fluctuación al aproximarnos al extremo abierto, se observa fácilmente. Sin embargo, al aumentar el régimen de giro a $3000 \mathrm{rpm}$ (figura 2.12(b)) puede verse como aunque esta tendencia sea todavía observable, las medidas están afectadas por la presencia de ruido, hecho que debe atribuirse a las fluctuaciones de turbulencia local, si bien la contribución pulsante domina todavía sobre la fluctuación de turbulencia local. Al aumentar el régimen de giro a $6000 \mathrm{rpm}$ la componente de ruido adquiere mayor importancia, como se muestra en la figura 2.12(c), siendo ahora ambas contribuciones a la fluctuación del flujo (pulsante y fluctuación turbulenta) del mismo orden de magnitud, independientemente de la posición del transductor de medida de presión en la cánula. La influencia que tales fluctuaciones provocan en los resultados que obtendremos al aplicar el método, se discutirá posteriormente en la sección 2.8, ya que en principio, esta componente no será propagada y radiada al exterior.

Una vez estimada la fluctuación del caudal volumétrico en la boca de la cánula de escape nos encontramos con la imposibilidad de poder contrastarla con resultados experimentales, ya que no existe en este caso comparación directa disponible. Sin embargo, la consistencia del procedimiento queda 
constatada en las figuras 2.13(a) y 2.13(b), donde se observa la disminución esperada de la amplitud de fluctuación al aumentar el régimen de giro y un aumento de las contribuciones de fluctuaciones de más alta frecuencia sobre la fluctuación del caudal volumétrico a medida que aumenta el régimen de giro, como se muestra en la figura 2.13(b). Ambos aspectos son consistentes con las tendencias observadas en las medidas de presión, ver figura 2.12.
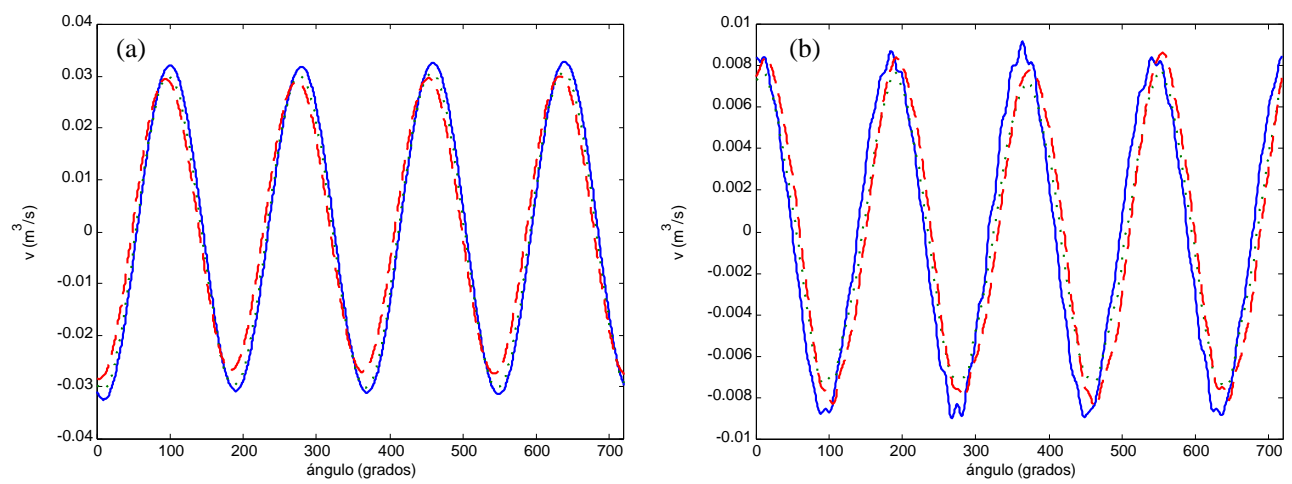

Figura 2.13: Caudal volumétrico estimado en el extremo abierto para un trasductor posicionado a $0.2 \mathrm{~m}$ (linea continua), a $0.15 \mathrm{~m}$ (linea punteada) y a $0.1 \mathrm{~m}$ (linea discontinua) a: $1500 \mathrm{rpm}$ (a) y $3000 \mathrm{rpm}$ (b).

Con el objetivo de obtener una mayor evidencia de la consistencia del método de estimación propuesto, a continuación se diagnosticará el efecto del cambio en la geometría de la línea de escape sobre el ruido emitido.

Como ya se comentó, la onda de presión acústica radiada al campo libre es proporcional a la derivada de la fluctuación del caudal volumétrico en el extremo abierto. Por tanto, las diferencias entre los niveles de presión sonora medidos para dos líneas de escape reales, puede ser estimada asumiendo que esta diferencia de nivel se reduce a la diferencia de espectros de $\dot{v}(t)$ para ambas disposiciones de escape, independientemente del modelo de emisión considerado. De este modo las incertidumbres asociadas con cualquier modelo de emisión o incertidumbres en las medidas de ruido son eliminadas, ya que todas las medidas se verían afectadas aproximadamente de la misma manera por estos factores, lo que permite evaluar el grado de precisión del procedimiento de estimación de $\dot{v}(t)$ dentro de la predicción del ruido de escape. 


\subsubsection{Análisis de la influencia del error del procedimiento pro- puesto en la estimación del ruido}

Las medidas del ruido de escape fueron realizadas con una sonda de intensidad cuyo error experimental asociado está en torno a $\pm 1 \mathrm{~dB}$, ver apéndice C. Por ello, y considerando la teoría de propagación de errores, la incertidumbre en la determinación de la diferencia de niveles sonoros, $\triangle S P L$, es aproximadamente de $\pm 2 \mathrm{~dB}$.

De forma similar se estimará el error cometido en la determinación de $\triangle S P L$ a través de la estimación de las derivadas temporales de la fluctuación del caudal volumétrico en la boca de escape. En este caso, para calcular el error real en la estimación de la derivada de la fluctuación de velocidad, es necesario añadir al $\pm 5 \%$ de error obtenido a través del análisis teórico descrito en la sección 2.3.2, el error asociado al método de calibración seguido en los transductores de presión, empleando la ecuación (2.31). Se considera que el error relativo cometido por el método de calibración en la medida de la presión acústica es de $\pm 0.7 \%$ para cada transductor, como señalan Piñero et al. [138], asumiendo una respuesta cuasi-estacionaria del transductor al medir la fluctuación de presión en el sistema de escape. De este modo el valor del error máximo alcanzado en este proceso de estimación será finalmente de $\pm 6 \%$ ( $\pm 0.8 \mathrm{~dB})$ para cada estimación de la fluctuación del caudal volumétrico, lo cuál implica un error máximo en la estimación del $\triangle S P L$ de $\pm 1.6 \mathrm{~dB}$, comparable con los $\pm 2 \mathrm{~dB}$ anteriores.

En las figuras 2.14 y 2.15 se representan los resultados del estudio llevado a cabo para 1000 y $3000 \mathrm{rpm}$, donde se consideran las diferencias de nivel de presión sonora, $\triangle S P L$, calculada a partir de las señales de presión medidas por los tres transductores de presión emplazados en la cánula de escape y las obtenidas a través de las medidas de ruido realizadas simultáneamente en una cámara semianecoica. Estos resultados se utilizarán para evaluar la sensibilidad del método a cambios en la línea de escape, permitiendo analizar la influencia en el método propuesto de la posición de los sensores de medida de presión en conductos.

Se puede observar en estas figuras, 2.14 y 2.15, un buen acuerdo para ambos regímenes de giro, para los cuatro primeros armónicos de motor desde H2 a H8 (frecuencias de encendido), para todas las posiciones de medida de presión consideradas. Este hecho garantiza la potencia de nuestro método si se tiene en cuenta que estos armónicos son los que contienen la información más relevante para la estimación del ruido global radiado al exterior. Por otra parte, para el resto de armónicos se obtiene un acuerdo global aceptable, lo 


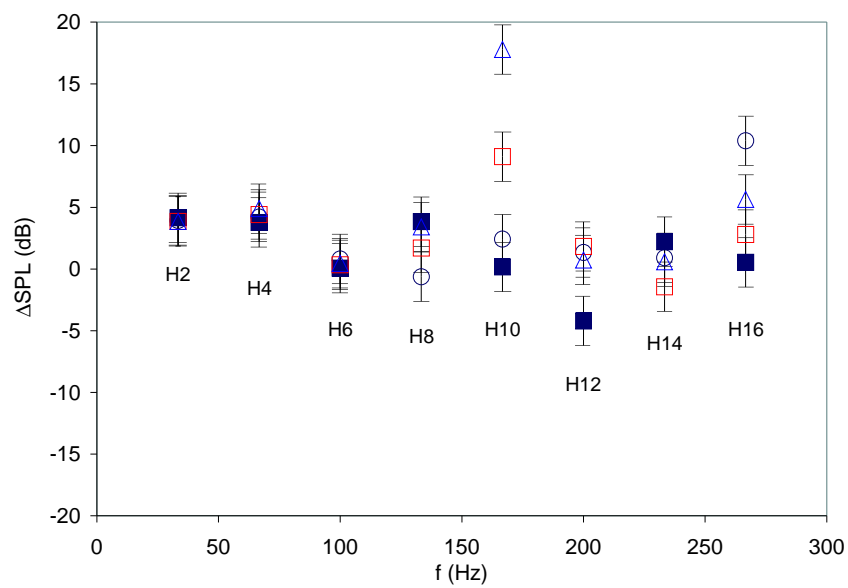

Figura 2.14: Diferencia de nivel de presión sonora a 1000 rpm entre dos lineas de escape en las que cambia el silenciador trasero: medidos (ద) y estimados para la medida de presión a $0.1 \mathrm{~m}(\bigcirc)$, a $0.15 \mathrm{~m}(\triangle)$ y a $0.2 \mathrm{~m}$ ( $\square)$.

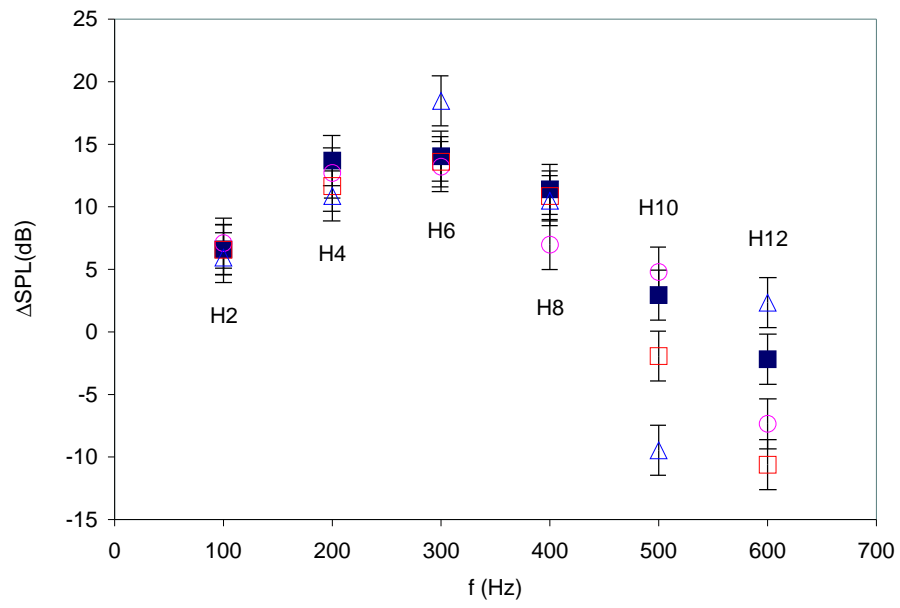

Figura 2.15: Diferencia de nivel de presión sonora a 3000 rpm entre dos lineas de

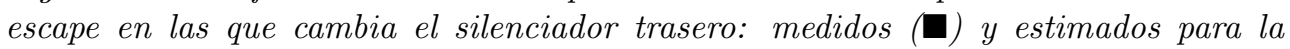
medida de presión a $0.1 \mathrm{~m}(\bigcirc)$, a $0.15 \mathrm{~m}(\triangle)$ y a $0.2 \mathrm{~m}$ ( $\square)$. 
cual ratifica la sensibilidad del método para la obtención del comportamiento espectral del ruido emitido.

Con respecto a la posición del transductor de medida de presión en la cánula, puede extraerse de estos resultados que su mejor posición para la aplicación del procedimiento depende del régimen de giro del motor; en general, el transductor debe alejarse del extremo abierto a medida que el régimen de giro aumenta. Así en la figura 2.14, puede observarse que la mejor reproducción de la diferencia del nivel sonoro medido con la sonda a $1000 \mathrm{rpm}$ se obtiene para la medida de presión a $0.1 \mathrm{~m}$ y $0.15 \mathrm{~m}$ antes del extremo abierto, para armónicos superiores H8, ya que para los cuatro primeros armónicos la estimación es igualmente buena en las tres posiciones. Esta mejor estimación a $0.1 \mathrm{~m}$ se debe al hecho de que este punto está lo suficientemente cerca del extremo abierto y por ello las hipótesis hechas para la propagación en el conducto son más válidas en este caso.

Cuando el régimen de giro aumenta la influencia de la fluctuación de ruido está afectando más a las señales cercanas al extremo abierto, ya que la amplitud de la fluctuación de presión decrece a medida que el punto de medida se aproxima al extremo abierto, ver figura 2.12(b). Esto concuerda con que la mejor estimación a $3000 \mathrm{rpm}$ al aumentar la frecuencia sea la obtenida para las medidas realizadas a $0.15 \mathrm{~m}$ del extremo abierto. Hecho que se debe fundamentalmente a dos factores: por un lado en este punto se tiene una mejor relación señal-ruido que en un punto más próximo a la boca de salida, y por otro lado las hipótesis del modelo siguen siendo válidas.

\subsection{Evaluación del modelo de emisión}

En la sección anterior se ha presentado un método de cálculo que permite la estimación de la fluctuación del caudal volumétrico en la boca de escape $v$, cuya viabilidad se ha puesto de manifiesto mediante la evaluación de su consistencia y precisión. Una vez conocida esta magnitud (fluctuación del caudal volumétrico), es necesario considerar un modelo de emisión para la estimación del espectro de ruido radiado, trabajando con hipótesis que nos aproximen al caso real. A través de los estudios realizados sobre la relación entre la fluctuación de la velocidad volumétrica y el ruido radiado $[16,136]$, se muestra la complejidad del fenómeno físico que tiene lugar en la boca de la cánula de escape como consecuencia de la interacción entre el fluido que sale de la misma y la atmósfera exterior, lo que se considera la fuente acústica de la componente de ruido que denominamos aerodinámico. 
De modo que, así, como en los apartados anteriores nos centramos principalmente en el problema de la fuente pulsante, considerando que es la fluctuación del flujo en la boca de la cánula la que se comporta como fuente acústica, en esta sección nos centraremos en el problema de la propagación y emisión de ruido, discutiendo los diferentes modelos de emisión empleados, es decir, aquellos modelos que permiten calcular el nivel de presión sonora o intensidad radiada a una cierta distancia de la boca de la cánula de escape, a partir de la variación temporal del gasto en ella, como último punto para resolver el problema de predicción de la componente pulsante del ruido de escape.

En relación a las limitaciones que presentan los modelos de emisión existentes se mencionan a continuación dos aspectos fundamentales de ellos, con el objetivo de mejorarlos:

- En primer lugar, estos modelos suelen emplearse para calcular el ruido radiado en campo libre (a grandes distancias de la fuente), por lo que se pierde información importante sobre las peculiaridades del flujo, alrededor de la boca de la cánula de escape.

- En segundo lugar, estos modelos no reproducen la complejidad de los fenómenos físicos que ocurren durante el proceso de radiación sonora en el escape del motor.

En este intento de descripción de los fenómenos fluidodinámicos responsables de la generación del ruido es necesario mencionar el obstáculo experimental encontrado, debido a la falta de herramientas adecuadas para poder visualizar y cuantificar estos fenómenos asociados a la descarga del flujo, tanto pulsante como turbulento. En especial este segundo tipo de técnica, de cuantificación, como la de identificación de modelos, han sido desarrolladas para validar modelos de emisión para diferentes tipos de fuentes, tanto en lo referente a su magnitud como a su patrón de direccionalidad, como se puede extraer de los trabajos de Filippi et al. [65], Nuttal y Wilson [129] y Reibold [147]. Trabajos, que sin embargo, no son aplicables al fenómeno de escape del motor donde la fuente sonora no es exactamente compacta, además de que su comportamiento y localización no son conocidos en detalle, aunque gran parte de los autores coinciden en situarla cerca de la boca de escape en la dirección del flujo [92].

En lo que se refiere a técnicas de visualización, se encuentran en la literatura algunas referencias a la aplicación de la fotografía de Schlieren, basada en la variación del índice de refracción del fluido con su densidad [171], de 
entre los que podemos resaltar trabajos como los de Blair y Coates [16], Henderson y Powell [73] y Umeda and Ishii [171]; sin embargo, la mayor parte de ellos se refieren a la emisión y excitación acústica por parte de un chorro. Otra técnica de visualización empleada es la de velocimetría de imágenes de partículas $P I V$ [107], que proporciona imágenes de distribuciones instantáneas de velocidad sobre un plano del flujo, siendo necesario dopar el ambiente con partículas visibles por la cámara empleada, ya que directamente estas cámaras no son capaces de detectar las partículas del gas, partículas que además deben poseer un tamaño relativamente grande para poder aumentar la densidad de la imagen y por tanto su resolución. A partir de este tipo de imágenes es posible analizar y describir el proceso físico que ocurre durante la descarga no estacionaria en la sección de salida del sistema de escape.

En la actualidad esta falta de información experimental está siendo sustituída por cálculos computacionales para evaluar el proceso de emisión. Sin embargo, a pesar de que esta es la línea más explotada hoy en día, todavía no está suficientemente desarrollada, aunque pueden resaltarse las soluciones analíticas obtenidas por Lee y Koo [96] basadas en modelos de expansión asintótica y aquéllas basadas en cálculos CFD (Computational Fluid Dynamics) para la evaluación del comportamiento de las diversas fuentes generadoras de ruido (Tam [162]). El empleo de este tipo de técnicas puede proporcionar ideas relevantes sobre los parámetros fundamentales y modelos envueltos en el proceso de emisión en el escape.

De acuerdo con los resultados publicado en la literatura [17], [74] y que resume Torregrosa en su Tesis Doctoral [168], se pueden distinguir tres fases en el proceso de descarga, las cuales llevan asociados mecanismos de generación de ruido diferentes:

1. La llegada del pulso de escape al extremo de la cánula, pulso aproximadamente uniforme en toda la sección que indica una transmisión de este pulso por el conducto esencialmente unidimensional. Cuando este pulso entra en la atmósfera, inicialmente se comporta como un pulso de presión que conserva las características de una onda plana que se propaga como si el tubo estuviese prolongado. Posteriormente la propagación de este pulso y su interacción con el fluido en reposo de su entorno hacen que se deforme en una onda tridimensional, generalmente esférica, que se va definiendo con más claridad a medida que se aleja de la fuente. Este frente es el responsable del ruido asociado a las oscilaciones del caudal en la boca debidas a las perturbaciones de presión que viajan por el conducto de escape. 
2. Al mismo tiempo que se propaga este frente de presión, se configuran estructuras de vorticidad en el entorno de la boca de la cánula de escape, debidas a los efectos de desprendimiento de vena fluida en ella, y la interacción producida entre el pulso emergente y el fluido exterior en reposo. Produciendo, por tanto, un movimiento claramente rotacional de las partículas del fluido en los bordes de la cánula, que se manifiesta finalmente en forma de vórtice toroidal. La presencia de este vórtice lleva asociada su correspondiente variación en el tiempo de los parámetros del flujo y representa por tanto una fuente sonora secundaria.

3. Este vórtice al separarse de la boca en su desplazamiento hacia el exterior, deja tras de sí una estela fuertemente turbulenta, que a su vez determina rápidas variaciones en el flujo y, consiguientemente, una componente adicional de naturaleza turbulenta al ruido emitido.

Se puede sintetizar esta descripción diciendo que existen al menos tres mecanismos de generación sonora asociados al fenómeno de descarga no estacionaria durante el proceso de escape. De los cuales, únicamente el primero, es decir, la generación del frente de onda esférico generado en la interacción del pulso incidente con el extremo, tiene una relación directa con el flujo en el interior del colector. Esta descripción del fenómeno nos da una idea de la dificultad que supone el tener en cuenta los fenómenos adicionales, fenómenos 2 y 3 .

La mayor parte de la literatura dedicada al problema de la radiación por el extremo de un conducto, se limita al caso en que las perturbaciones incidentes sobre dicho extremo sean de pequeña amplitud, caso en el que sí que es posible resolver simultáneamente, con diversos grados de aproximación, las ecuaciones correspondientes al flujo en el interior de la cánula de escape y en el exterior de la misma. De esta forma y a través de técnicas matemáticas como el método de Wiener-Hopf [23], es posible obtener soluciones analíticas para el campo de presiones asociado a la radiación en el espacio libre, como comentan autores como Cargill [22], y Norris y Sheng [128]. Además de estas técnicas existe un segundo grupo de soluciones que parten de la forma más general de la ecuación integral de Hemholtz [121]. Los resultados derivados de estos estudios muestran la existencia de tres contribuciones a la radiación, que aparecen de forma natural como consecuencia de aplicar la solución de Kirchhoff [121] a la ecuación integral planteada por Crighton [32] para la obtención del ruido radiado al campo libre por una fuente $q$ a una distancia $r$ de la fuente, $\int 1 /(4 \pi r) q d^{3} \mathbf{r}$, y que pueden resumirse como: 
1. Un primer término, que corresponde a un conjunto de fuentes simples isótropas, representadas por una cierta distribución de monopolos, caracterizadas por la derivada del caudal asociado a ellos [95] [104]. Esta emisión está directamente relacionada con la variación temporal de la masa presente en la región del espacio considerada como fuente acústica.

2. Un segundo término asociado a la variación de la cantidad de movimiento existente en esta región fuente. Esta variación es interpretada como el resultado de la interacción de dos fuentes simples de igual magnitud y signo contrario, que determina la existencia de un flujo oscilante entre ambas que se asocia un dipolo acústico y por tanto se considera como una componente dipolar de la radiación.

3. Un tercer y último término que está relacionado con la posible variación de la cantidad de movimiento del flujo, ya que se produce a una cierta distancia de cualquier superficie sólida, donde la masa y la cantidad de movimiento de una región fija del espacio no varían. Debido a este hecho, el flujo de la cantidad de movimiento debe cancelarse, pudiéndose interpretar este término como el resultado de la interacción de pares de dipolos acústicos, manifestándose así como una contribución cuadripolar al campo de radiación.

$\mathrm{Al}$ considerar el campo de radiación como superposición de estas tres contribuciones, parece conveniente poder asociar estos tres patrones de radiación a los tres mecanismos de generación de ruido mencionados al comienzo de la sección. Este punto no se encuentra tratado con profundidad en la literatura, ya que la evidencia experimental es escasa y referida a unas condiciones muy particulares de medida. Sin embargo, sí es posible aventurar algunas consideraciones al respecto. El término monopolar parece fácilmente atribuible a la propagación de la onda esférica, ya que por definición la radiación procedente de una fuente simple es isótropa y por tanto, debe manifestarse como una onda esférica que se aleja de la fuente. En este sentido la interpretación del término dipolar resulta más problemática, aunque puede asociarse a la existencia del vórtice toroidal en la boca de la cánula de escape con una variación periódica de la cantidad de movimiento en los puntos de su entorno. Por último, el término de radiación cuadripolar admite una interpretación en términos de fluctuaciones turbulentas, tal como manifiesta la teoría de Lighthill [102], acerca del sonido emitido en una región del espacio en la que existe un flujo turbulento totalmente desarrollado.

Bien es cierto que, aunque en principio parezca posible calcular aisladamente los tres términos descritos, dicho cálculo no es viable en la práctica 
debido a la dificultad que comporta la evaluación de las correspondientes integrales. Es por ello que el cálculo se reduce generalmente al estudio de la primera contribución, con lo cuál es posible perder información relevante sobre todo a altos regímenes de giro, en los que los fenómenos de vorticidad y turbulencia tienen mayor presencia [93]. Esta es la razón por la que se proponen diferentes modelos de emisión a fin de que contengan los efectos derivados de estas fuentes de ruido secundarias, siendo evaluada la validez de los mismos mediante la comparación directa con las medidas de ruido disponibles.

Estos modelos pretenden describir el proceso de descarga, fenómeno de interacción de la fluctuación de velocidad existente en dicha sección y las condiciones de la atmósfera exterior, causa de la emisión de ruido aerodinámico, partiendo de la afirmación generalizada de que esta radiación sonora es directamente proporcional a la aceleración del volumen de la fuente [58]. Por tanto, todos los modelos partirán del mismo factor común: fluctuación de la aceleración del volumen del fluido en la boca del escape (sección de descarga) como fuente acústica [136], la cuál es perfectamente conocida como se ha mostrado en las secciones anteriores.

\subsection{Modelo monopolar}

Como se ha mencionado el ruido emitido debido a la presencia de un flujo no estacionario y una onda pulsante en el sistema de escape se debe principalmente a la interacción del flujo y las condiciones exteriores. Esta interacción es descrita normalmente en términos de un modelo de emisión simple (fuente monopolar) [95] correspondiente a una fuente isótropa caracterizada por el mismo caudal volumétrico que existe en el extremo abierto. Por tanto esta forma de emisión será analizada como primera aproximación al problema de emisión del escape de un motor real.

Esta forma de abordar el problema de la emisión del sonido procedente de un extremo abierto, radica en la consideración de que la diferencia de presiones entre el gas en el extremo del tubo y el que está en el espacio que le rodea es pequeña en comparación con la diferencia de presiones dentro del propio tubo. De aquí que la condición límite en el extremo abierto del tubo es, con suficiente exactitud, que la fluctuación de presión debe ser nula. Sin embargo la velocidad del gas $\widetilde{u}$ en el extremo del tubo no es cero, por tanto el volumen de gas que sale del tubo por unidad de tiempo se corresponde con el producto $A_{s} \widetilde{u}$, donde $A_{s}$ representa la sección de la cánula de salida. Esto permite considerar el extremo abierto de un tubo como una 'fuente' sonora de intensidad $A_{s} \widetilde{u}$. Tal fuente se concibe como una pequeña esfera pulsante, de 
volumen variable con el tiempo y correspondiente a una radiación isótropa. Esta variación de volumen origina el movimiento del fluido inmediato a su superficie y, por tanto, una fluctuación de la masa del fluido contenido en dicha región del espacio, de la misma forma que ocurre con la radiación del término monopolar en el extremo. Como resultado de tal variación el volumen de fluido se ve expelido o bien aspirado durante las pulsaciones que sufre el cuerpo emisor.

El problema de la emisión del extremo de un tubo se hace así equivalente al de emisión de un cuerpo pulsante, para el que la intensidad del sonido radiado resulta ser proporcional al cuadrado de la aceleración de variación del volumen del fluido con el tiempo, $A_{s} \dot{\widetilde{u}}$, esto es [95]:

$$
I_{T}=\frac{\rho_{o} A_{s}^{2} \dot{\widetilde{u}}^{2}}{4 \pi a_{o}}
$$

Teniendo en cuenta que la emisión en este caso resulta ser independiente de la dirección de emisión, es decir, emisión isótropa, la intensidad radiada por unidad de superficie a una distancia $r$ de la fuente será, según Lighthill [104]:

$$
I_{r}(r)=\frac{\rho_{o} A_{s}^{2}}{a_{o}} \cdot \frac{\dot{\widetilde{u}}^{2}}{(4 \pi r)^{2}}
$$

o equivalentemente, la variación temporal de la presión acústica en este mismo punto viene dada por [95]

$$
p(r, t)=\frac{\rho_{o} A_{s}}{4 \pi r} \cdot \dot{\widetilde{u}}\left(t-\frac{r}{a_{o}}\right)
$$

donde $\rho_{o}$ y $a_{o}$ representan la densidad y la velocidad del sonido a temperatura ambiente. Obsérvese que la presión sonora es finalmente proporcional a la derivada de la velocidad en la boca, siendo ésta calculada en el instante retardado $\left(t-\frac{r}{a_{o}}\right)$ dando cuenta de la velocidad finita de propagación de la onda esférica en el espacio libre, la cual se obtiene de forma natural cuando se aplica rigurosamente la ecuación de Kirchhoff [121].

Desde el punto de vista experimental, la aparición de la derivada temporal de la velocidad en la boca (aceleración del volumen del fluido) añade una nueva indeterminación en los resultados que puedan obtenerse, ya que no se dispone de la forma analítica de la variación de la velocidad con el tiempo, sino únicamente de un número finito de muestras. Sin embargo, debido al carácter periódico de la velocidad, su derivada $\dot{\widetilde{u}}(t)$ es fácil de calcular haciendo uso de la definición de la transformada de Fourier discreta. De este modo 
obtendremos el valor de la derivada en dominio frecuencia, lo cual permite obtener directamente el espectro del nivel de presión sonora en el punto del espacio considerado, magnitud de mayor interés que la evolución temporal de la propia presión sonora desde el punto de vista del ruido radiado.

El resultado de dicho cálculo, en términos de intensidad sonora radiada asociada a una determinada frecuencia angular $\omega$ [104] es

$$
I_{r}(r, \omega)=\frac{\rho_{o} \omega^{2}}{a_{o}} \cdot \frac{\left(A_{s} \cdot \widetilde{u}(\omega)\right)^{2}}{(4 \pi r)^{2}}
$$

donde $\widetilde{u}(\omega)$ representa la amplitud compleja de la fluctuación de velocidad en el extremo de la cánula asociada a la frecuencia angular $\omega$.

Una vez conocido el valor de la presión e intensidad acústica radiada para calcular el nivel de presión sonora o de intensidad sonora (magnitudes equivalentes en condiciones de campo libre) es necesario considerar la amplitud de la presión eficaz, $p_{r m s}(r, \omega)=p(r, \omega) / \sqrt{2}$, ya que generalmente el nivel de presión sonora se define en términos de ella, traduciéndose en la siguiente expresión para la intensidad acústica radiada

$$
I_{r m s}(r, \omega)=\frac{\rho_{o} \omega^{2}}{2 \cdot a_{o}} \cdot \frac{\left(A_{s} \cdot \widetilde{u}(\omega)\right)^{2}}{(4 \pi r)^{2}}=\frac{\rho_{o} a_{o}}{2}\left(k a^{2}\right)^{2}\left(\frac{\widetilde{u}(\omega)}{4 r}\right)^{2}
$$

Finalmente, el nivel de intensidad sonora se calcula aplicando la ecuación (2.36), normalizando el resultado a una cierta intensidad de referencia y expresándolo en la escala logarítmica habitual $(\mathrm{dB})$. De forma análoga se obtendría el nivel de presión sonora.

\subsection{Modelo de emisión de un pistón vibrante}

Con el fin de considerar la influencia del flujo sobre el sonido radiado por un motor e incluirlo en el modelo de emisión a través de una eficiencia de radiación que dé cuenta del fenómeno, se asume que el sonido radiado tiene alguna característica direccional [16], y por tanto el modelo simplificado de radiación isótropa pudiera no ser adecuado para describir el proceso real de emisión. Autores como Comparin [29] mantienen que el modelo de fuente monopolar puede emplearse para estimar la intensidad acústica emitida en presencia de flujo medio no nulo sólo si la fuente es compacta, su localización es conocida y además la posición de medida está a una distancia adecuada de 
la fuente. Sin embargo, estas hipótesis no son exactamente reproducidas en el escape de un motor, donde la presencia de flujo medio altera los gradientes de presión aguas arriba y aguas abajo de la fuente y por tanto ni la fuente es compacta ni su localización está perfectamente definida.

Como solución al proceso de emisión y con la idea de encontrar un comportamiento físico más realista del extremo abierto, de acuerdo con las ideas defendidas por Rayleigh [145], se considera que este se comporta de forma similar a un pistón oscilante emplazado en una pared infinita. Este autor afirma que si la causa de la perturbación dentro del tubo es el paso de un tren de ondas progresivas a través del extremo abierto cuyo diámetro es inferior a la longitud de onda de la vibración considerada, es posible asumir que la fuente de movimiento de las ondas de presión dentro del tubo se deben al movimiento de un pistón en el extremo abierto. Esta situación es muy aproximada a la del pulso que recorre el sistema de escape y a la no existencia de una velocidad constante del gas a través de la sección de salida [15].

Si asumimos que la longitud de onda es mucho mayor que el diámetro del tubo de escape, por lo que la única onda que puede propagarse en el interior del conducto es una onda plana, en su modo fundamental, es posible ignorar la no uniformidad de la distribución de velocidad a través de la sección transversal del conducto y asumir que la fluctuación del caudal en el extremo abierto actúa como un pistón, radiando sonido al exterior y reflejando parte de la energía hacia el silenciador trasero. A medida que el régimen de giro aumenta la frecuencia característica aumenta y mayor proporción de energía se propaga hacia el exterior.

Por todo ello, se estudia el modelo de emisión sonora originado por el movimiento de un pistón circular plano, de masa cero y radio $a$, fijo en un plano de contorno $x=0$ (en el extremo abierto de la cánula), rodeado por una pared infinita, ver figura 2.16. Éste, se mueve en la dirección $x$, mediante un movimiento armónico simple, con velocidad $u_{x}=\widetilde{u}(\omega) \cdot \exp (-j \omega t)$ en el interior de la sección de salida del conducto de radio $a$, y cero en el resto del plano $x=0$.

Para la obtención de la presión sonora emitida en la dirección $\mathbf{x}$ por dicho pistón partimos del conocimiento de su gradiente, $\nabla p=-\rho_{o} \partial \mathbf{u} / \partial t$, dado por la ecuación de continuidad. Añadiendo, como condición de contorno, que posea un gradiente de presión normal a la superficie en la sección de salida de la cánula, de valor $j k \rho_{o} a_{o} \widetilde{u}(\omega) \cdot \exp (-j \omega t)$, y cero en el resto del plano (pared infinita) y considerando que el pistón está compuesto por un gran número de áreas elementales, de forma que el campo de presión resultante será la integral de superficie del sonido creado por cada área puntual del pistón que actúa 


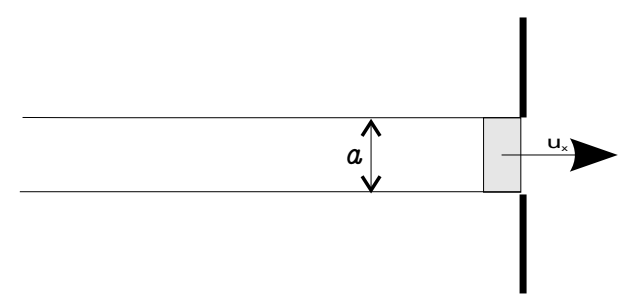

Figura 2.16: Esquema del movimiento de un pistón de radio a, fijo en un muro infinito en el plano de contorno $x=0$.

como una fuente simple de intensidad $|\widetilde{u}(\omega)| d x_{0} d y_{0}$, calculándose el campo de presión sonora mediante la siguiente ecuación

$$
p(r, \omega)=-j k \rho_{o} a_{o} \iint|\widetilde{u}(\omega)|\left[G_{\omega}\left(\mathbf{r} \mid \mathbf{r}_{0}\right)\right]_{z=0} d x_{0} d y_{0}
$$

De este modo el problema se traslada ahora al conocimiento de la expresión de la función de Green, $G_{\omega}\left(\mathbf{r} \mid \mathbf{r}_{0}\right)$, que resuelve la ecuación (2.37). Para ello, y tal y como explica Morse [121], se analiza el efecto que plantea la presencia de una barrera plana infinita, es decir, que el medio está confinado en un lado del plano, introduciendo la teoría de las imágenes. Esta teoría se basa en el reemplazamiento de la superficie de contorno por un medio continuo situado en su parte posterior y simétrico al medio de emisión, ver figura 2.17. En este nuevo medio se emplazan las imágenes de las fuentes sonoras, de modo que se anule el gradiente del campo de presión emitida en la superficie de contorno. De esta forma, la función de Green apropiada para calcular el campo de presión generado en $\mathbf{r}$ debido a una fuente puntual $P_{o}$ en $\mathbf{r}_{0}, G_{\omega}\left(\mathbf{r} \mid \mathbf{r}_{0}\right)$, debe contener la presión original asociada a la onda esférica originada por la fuente puntual $P_{o}$ y la onda reflejada que proviene de su fuente imagen $P_{o}^{\prime}$ en $\mathbf{r}_{0}^{\prime}$, anulándose automáticamente en $x=0 \mathrm{y}$ haciendo que la presión sea justo el doble que si el contorno no estuviese presente. La expresión final de esta función de Green es

$$
G_{\omega}\left(\mathbf{r} \mid \mathbf{r}_{0}\right)=\frac{\exp (-j k R)}{4 \pi R}+\frac{\exp \left(-j k R^{\prime}\right)}{4 \pi R^{\prime}}
$$

Si la superficie de contorno en $x=0$ no es perfectamente rígida y reacciona a la onda de presión incidente mediante una impedancia acústica $Z(\omega)=\rho_{o} a_{o} / \beta_{s}$, como es el caso del extremo abierto de la cánula de escape, y cualquier porción del plano se está moviendo, el término imagen ve modificada 


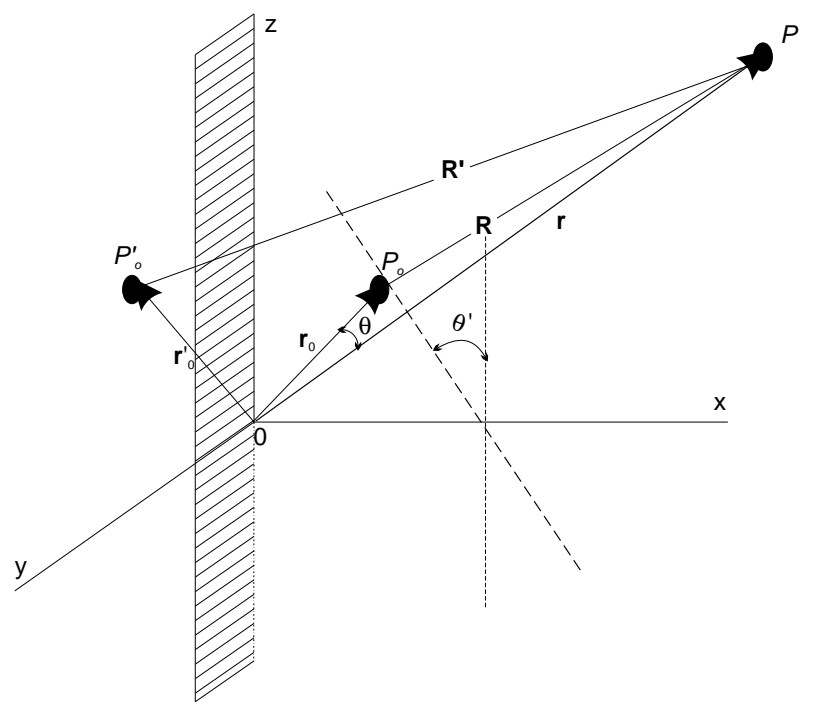

Figura 2.17: Distancias y ángulos para un punto $P$ del campo libre al punto fuente Po y a su imagen $P^{\prime}$.

su función de Green por este factor, quedando

$$
G_{\omega}\left(\mathbf{r} \mid \mathbf{r}_{0}\right) \simeq \frac{\exp (-j k R)}{4 \pi R}+\frac{\cos \theta^{\prime}-\beta_{s}}{\cos \theta^{\prime}+\beta_{s}} \frac{\exp \left(-j k R^{\prime}\right)}{4 \pi R^{\prime}}
$$

A partir de esta expresión es posible calcular el campo de radiación creado por una fuente multipolar en presencia de una superficie plana de admitancia $\beta_{s}$.

Cuando la distancia $r$ desde la región fuente en el contorno es mucho mayor que $a$, el radio del circulo que encierra el área fuente, la función de Green tiene una expresión más simple, a través de la consideración de que $R^{\prime} \approx R, \mathrm{y} \theta^{\prime} \approx \theta$.

$$
G_{\omega}\left(\mathbf{r} \mid \mathbf{r}_{0}\right) \simeq \frac{\exp (-j k R)}{4 \pi R}\left[1+\frac{\cos \theta-\beta_{s}}{\cos \theta+\beta_{s}}\right]=\frac{\exp (-j k R)}{2 \pi R}\left[\frac{\cos \theta}{\cos \theta+\beta_{s}}\right]
$$

Finalmente, si se sustituye la función de Green (2.40) en la ecuación (2.37) se obtiene un campo de presión sonora de la forma

$$
p(r, \omega, \theta)=-j k \rho_{o} a_{o}\left(\frac{\cos \theta}{\cos \theta+\beta_{s}}\right) \iint|\widetilde{u}(\omega)|\left[\frac{\exp (-j k R)}{2 \pi R}\right]_{z=0} d x_{0} d y_{0}
$$


Cambiando a coordenadas polares e integrando esta ecuación (2.41) sobre la superficie del pistón se obtiene

$$
p(r, \omega, \theta)=-j k \rho_{o} a_{o}|\widetilde{u}(\omega)|\left(\frac{\cos \theta}{\cos \theta+\beta_{s}}\right) \int_{0}^{a} \varrho d \varrho \int_{0}^{2 \pi}\left[\frac{\exp (-j k R)}{2 \pi R}\right]_{z=0} d \psi
$$

Haciendo uso de la ley de los cosenos y la relación trigonométrica entre ángulos complementarios, $\cos (\pi / 2-\theta)=\sin \theta$, se llega a la expresión de $R$ como: $R^{2}=r^{2}+\varrho^{2} \cos ^{2} \psi-2 r \varrho \cos \psi \sin \theta$. Para el caso del campo lejano, $\varrho / r \ll 1$, pudiendo ser despreciados los términos de segundo orden y emplear la siguiente fórmula de aproximación, $(1+x)^{1 / 2} \simeq 1+x / 2$ para $x \ll 1$, obteniendo:

$$
R \simeq r-\varrho \cos \psi \sin \theta
$$

En este punto puede asumirse que el efecto del factor de corrección, - $\varrho \cos \psi \sin \theta$, con la distancia es importante únicamente para el ángulo de fase de una onda pudiendo ser despreciado su efecto sobre la amplitud. Bajo este criterio el campo de presión sonora en el campo libre es

$$
\begin{aligned}
p(r, \omega, \theta)= & -\frac{j k \rho_{o} a_{o}}{2 \pi r}|\widetilde{u}(\omega)| \exp (-j k r)\left(\frac{\cos \theta}{\cos \theta+\beta_{s}}\right) \\
& \int_{0}^{a} \varrho d \varrho \int_{0}^{2 \pi} \exp (j k \varrho \cos \psi \sin \theta) d \psi
\end{aligned}
$$

Teniendo en cuenta las siguientes propiedades de las funciones de Bessel $J_{n}(z)$ :

$$
\begin{gathered}
J_{n}(z)=\frac{1}{2 \pi} j^{n} \int_{0}^{2 \pi} \exp (j z \cos \phi) \cos (n \phi) d \phi \\
\int z J_{0}(z) d z=z J_{1}(z)
\end{gathered}
$$

Se obtiene la siguiente expresión para el campo de la presión sonora radiada

$$
p(r, \omega, \theta)=-j k \rho_{o} a_{o} \widetilde{u}(\omega) \frac{\exp (-j k r)}{2 \pi r} \pi a\left[\frac{\cos \theta}{\cos \theta+\beta_{s}} \frac{2 J_{1}(k a \sin \theta)}{(k a \sin \theta)}\right]
$$

Reordenando los términos anteriores se obtiene la presión radiada al campo libre [121], que contiene el efecto de la admitancia $\beta_{s}$ del extremo abierto.

$$
p(r, \omega, \theta)=-j(K k) \rho_{o} a_{o} \frac{\exp (j(K k) r)}{2 r} a|\widetilde{u}(\omega)| f(k, \theta)
$$


En esta expresión $K=c / a_{o}$ es el cociente de las velocidades del sonido, que se emplea como parámetro corrector del número de onda acústico $(k=\omega / c)$ cuando se radia un flujo caliente [37], y $f(k, \theta)$ es el factor de distribución angular, el cual depende de la frecuencia y del ángulo polar $\theta$, y es próximo a la unidad en $\theta=0$, cuando $\beta_{s} \ll 1$, y cuya expresión es

$$
f(k, \theta)=\left[\frac{\cos \theta}{\beta_{s}+\cos \theta} \frac{2 J_{1}(K k a \sin \theta)}{(K k a \sin \theta)}\right]
$$

donde $J_{1}$ es la función de Bessel de primer orden, y $K k a$ es el número de Helmholtz corregido por el efecto del flujo caliente.

Finalmente, la intensidad sonora radiada al campo lejano a una distancia $r$ del extremo abierto de la cánula adopta la expresión

$$
I_{r m s}=\frac{1}{8} \rho_{o} a_{o}(K k a)^{2}\left(\frac{a}{r}\right)^{2}|\widetilde{u}(\omega)|^{2}[f(k, \theta)]^{2}
$$

De la comparación de esta última expresión (2.50) con la intensidad monopolar (2.36) se concluye que la única diferencia, a excepción de un factor constante derivado de considerar radiación en campo libre ó en un semiespacio delimitado por una pared rígida, es el factor de directividad de la intensidad radiada, $[f(k, \theta)]^{2}=D(\omega, \theta)$, cuyas características se comentarán a continuación. En primer lugar este término contiene el efecto de la impedancia del contorno, como ya se ha mencionado anteriormente, por medio de la $a d m i$ tancia específica de la superficie $\left(\beta_{s}\right)$, definida como $\beta_{s}=\rho_{o} c / Z(\omega)$, factor que puede obtenerse a través de la expresión del coeficiente de reflexión, haciendo uso de la relación existente entre la impedancia en el extremo $Z(\omega)$ y dicho coeficiente. De esta forma la obtención de la admitancia específica queda reducida al cálculo de la siguiente expresión:

$$
\beta_{s}=\frac{1-R(\omega)}{1+R(\omega)}
$$

Este factor da cuenta de la distorsión del campo próximo producido por el contorno [121], de modo que $\beta_{s}$ constituye un factor de peso para las diferentes velocidades de giro del motor y componentes frecuenciales del patrón de directividad, como se muestra en la figura 2.18. Ya que, de acuerdo con la ecuación 2.51 para una frecuencia dada el valor del coeficiente de reflexión se modifica al variar el régimen de giro y en consecuencia el valor de $\beta_{s}$ también se ve modificado. Esto hace que, fijado el régimen de giro, el patrón de directividad de la emisión vaya evolucionando desde formas de radiación más isótropas (contribución monopolar) a bajas frecuencias hacia un comportamiento más 


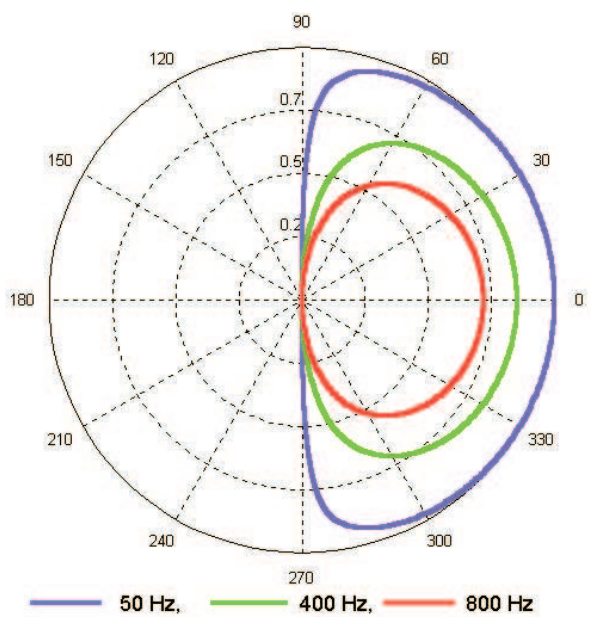

Figura 2.18: Diagrama polar del patrón de directividad a 1000 rpm.

dipolar, con lóbulos de radiación con máximos apreciables a $0^{\circ}$, a medida que el rango de frecuencias aumenta [59].

Por otro lado, además del cambio en el patrón de radiación también se observa un cambio en la eficiencia de radiación, disminuyendo ésta a medida que la frecuencia aumenta para un ángulo de observación fijo, como queda reflejado en la figura 2.19. Efecto que se corresponde con el patrón observado en los espectros de ruido de escape medidos, como se verá en las figuras 2.212.23 , lo que permite aventurar que esta eficiencia de radiación se ajusta más al problema de emisión real, dando cuenta del efecto de la interacción entre el flujo pulsante y la atmósfera en reposo [170]. Otra curiosa tendencia de los espectros medidos que reproduce el factor de directividad, es el efecto de menor disminución de la eficiencia de radiación a medida que aumenta la frecuencia al aumentar el régimen de giro del motor.

\subsection{Evaluación de los modelos frente a las medidas experimentales}

Vamos a afrontar a continuación la validación experimental de la técnica de estimación del ruido de escape expuesta en las secciones anteriores, ya que disponemos de información experimental directa y adecuada de este fenómeno. Los resultados presentados corresponden al mismo motor empleado en la validación del método de estimación de la fluctuación de velocidad en la sección de 


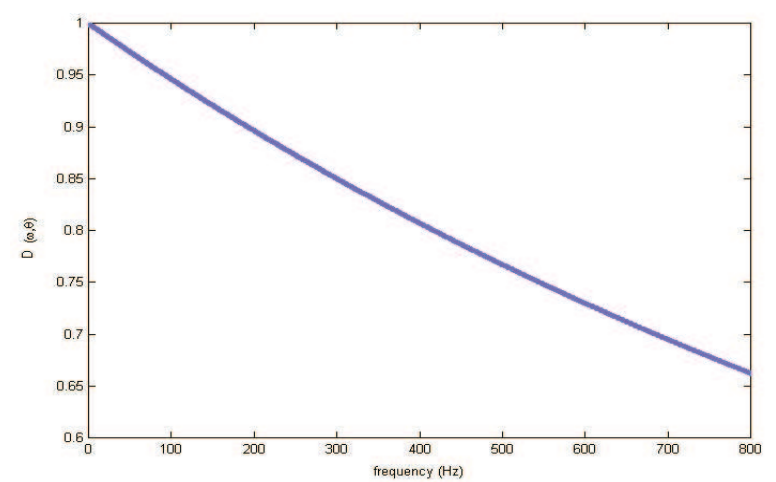

Figura 2.19: Comportamiento del factor de directividad a 1000 rpm para un posición de observación de $30^{\circ}$ con el eje horizontal

salida de la cánula de escape, cuyas dimensiones ya fueron detalladas entonces, por lo que ahora nos centraremos exclusivamente en una breve descripción de la instalación y de las condiciones semianecoicas empleadas para las medidas del ruido radiado. La finalidad es acotar la fiabilidad del procedimiento propuesto a través de la correlación directa entre estas medias de ruido y las estimaciones realizadas.

Es importante subrayar que la ventaja que se deriva de validar esta técnica de estimación con medidas realizadas en una cámara semianecoica es la de eliminar la influencia de toda fuente exterior, a excepción del efecto del fenómeno de la descarga que tiene lugar en la boca de la cánula de escape.

\subsubsection{Medida de ruido}

Como ya se comentó en el capítulo 1 , el proceso de medida de ruido es sumamente delicado debido al gran número de factores que deben controlarse y a la dificultad que conlleva este control [83]. Si además se tiene en cuenta que es una medida realizada en el dominio de la frecuencia cualquier imprecisión en su medida, selección de la frecuencia de muestreo y resolución frecuencial, puede producir resultados erróneos y por tanto de difícil interpretación. Esta problemática adquiere mayor importancia cuando el objetivo no es comparar una serie de medidas, donde todas ellas estarían afectadas por las mismas incertidumbres sino que, como en el caso que nos ocupa, se trata de obtener información para validar los resultados de un modelo de predicción, siendo ahora los parámetros del sistema de medida un factor crítico. Para 
que la medida del ruido de escape radiado sea de utilidad comparativa con los resultados de estimación obtenidos, se deben tener en cuenta las siguientes consideraciones [168]:

- La boca de escape debe hallarse convenientemente aislada del resto de las fuentes sonoras mencionadas en el capítulo 1: el ruido mecánico emitido por el bloque, el ruido de admisión y el ruido radiado por las vibraciones del colector de escape. Por ello, se debe aislar físicamente la boca del colector de escape del resto del motor, bien mediante el empleo de paneles aislantes o bien llevándola fuera de la sala del motor.

- Una vez aislada la boca del colector de escape, la medida debe realizarse en condiciones que puedan considerarse anecoicas, esto es, que la presión sonora emitida no se vea perturbada por su reflexión en superficies próximas a ella (suelo, paredes, obstáculos), ya que de esta manera se produciría una interferencia parásita que desvirtuaría los resultados.

- Resulta además conveniente que las condiciones ambientales durante la medida sean semejantes entre las medidas correspondientes a una misma serie, sobre todo si las medidas son promediadas para obtener un ciclo de ruido representativo. De lo contrario, a la inestabilidad propia del motor habría que añadir corrimientos frecuenciales adicionales, debidos a cambios en la temperatura, en la velocidad del viento (en el caso de que las medidas fueran realizadas en el exterior), etc.

Respecto al primer punto, una posible solución es el empleo de la intensimetría acústica, que debido a su carácter direccional aísla por sí misma una determinada fuente sonora [84]. Sin embargo, la intensimetría es una técnica laboriosa que requiere un número considerable de medidas para cada caso de estudio y, aunque su uso para la separación de fuentes está cada vez más extendido, la práctica habitual sobre todo en la industria, es el empleo de la sonometría, es decir el simple registro de la presión acústica en un punto del espacio, sin considerar su posible procedencia.

En lo que se refiere a la realización de las medidas en condiciones anecoicas, el empleo de las cámaras anecoicas no es una práctica muy generalizada debido a su alto coste. Por ello, en muchos casos se sustituye este tipo de instalación por medidas en un espacio abierto libre de superficies reflectantes. De esta forma el problema del aislamiento del motor y el ambiente anecoico se soluciona sólo parcialmente.

Las medidas de ruido que se utilizarán para nuestro diagnóstico experimental se realizan en una cámara semianecoica, subsanando así parte de los 
problemas antes mencionados. Esta cámara semianecoica se encuentra emplazada junto a una sala de ensayos de motor mostrada esquemáticamente en la figura 2.20.

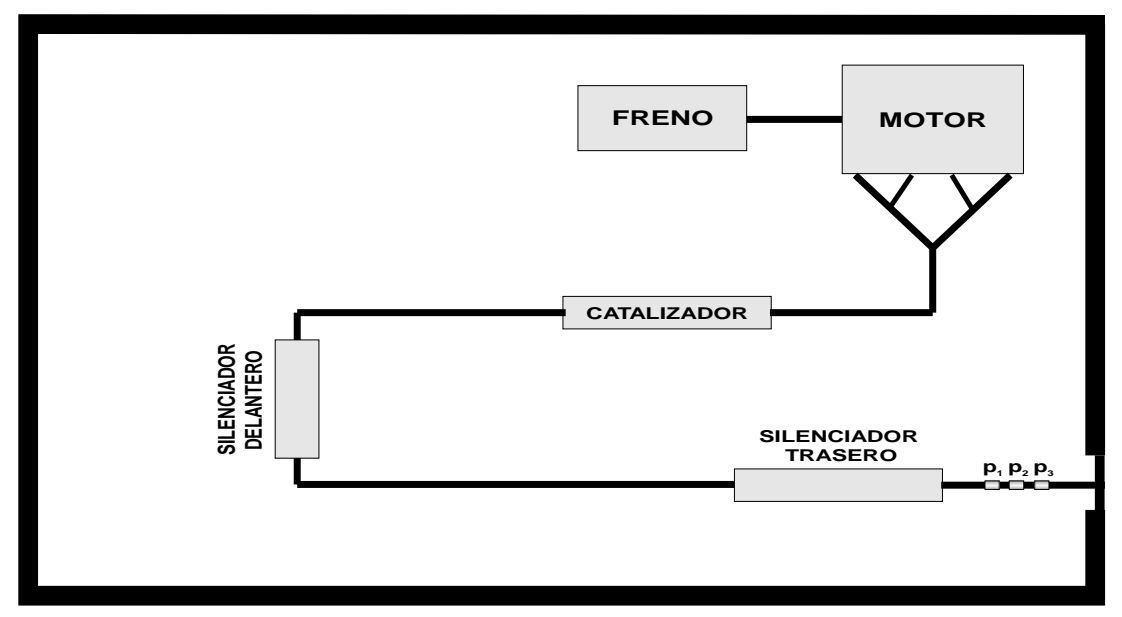

Figura 2.20: Esquema de la medida en motor policilíndrico.

En primer lugar, hay que señalar que la posición del motor en esta celda de ensayos permite emplear longitudes del colector similares a las que existen en condiciones reales de uso del motor, proporcionando disposiciones realistas de las líneas de escape. En segundo lugar, la boca de la cánula de escape se encuentra enrasada con la pared exterior de la sala, garantizando así el aislamiento de la boca de salida del escape del resto del motor mediante el uso de un pasamuros de $0.11 \mathrm{~m}$ de longitud (separación de la sala motor y cámara semianecoica), que funciona como pared aislante entre el ruido generado en la sala de ensayos y la cámara semianecoica. Se consigue de esta manera aislar la medida del ruido emitido, de cualquier fuente de ruido proveniente del motor que no sea el propio ruido de escape. Además, el hecho de que todo el colector se encuentre dentro de la sala de ensayos de motor también resulta ventajoso en cuanto a que las condiciones ambientales que afectan al flujo en el interior del mismo son las mismas.

Para las medidas del ruido emitido se empleó una sonda de intensidad, con las ventajas e inconvenientes que ello conlleva, explicadas con mayor profundidad en el apéndice C. Dicha sonda se posicionó a $1 \mathrm{~m}$ de la boca de emisión y en dos posiciones ángulares diferentes, $30^{\circ}$ y $45^{\circ}$ respecto al eje de la cánula de escape, con la finalidad de validar la técnica propuesta bajo condiciones de campo lejano (punto de medida suficientemente alejado de la fuente que gene- 
ralmente se acota por una longitud superior a la dimensión característica de la fuente, diámetro de sección de salida en este caso) y estudiar la dependencia angular de los modelos de emisión.

Se realizaron medidas adicionales, mediante micrófonos de $1 / 2$ " tipo 4190 Falcon, en otras posiciones según las normas PSA $\left(0.1 \sqrt{2} \mathrm{~m}, 45^{\circ}\right)$, Mercedes Benz $\left(0.225 \mathrm{~m}, 45^{\circ}\right)$ y Opel $\left(0.5 \mathrm{~m}, 45^{\circ}\right)$. A este respecto, si recordamos los complejos fenómenos que tienen lugar en las proximidades de la boca de escape, las distancias consideradas en las normas PSA y Mercedes Benz parecen algo cortas, pudiendo introducir dificultades adicionales en la predicción debido a los efectos mencionados, y con la posibilidad además de perturbar las condiciones del flujo por la presencia de micrófonos en dichas posiciones. Por ello, se han escogido los resultados obtenidos a $1 \mathrm{~m}$, para validar la técnica de predicción propuesta, ya que si bien es cierto que los resultados de la norma Opel serían igualmente válidos (hipótesis de campo libre), no se dispone de dos posiciones angulares en esta posición a fin de analizar el efecto de la directividad de la fuente.

Respecto a la cámara semianecoica, el tipo de material absorbente empleado para recubrir sus paredes laterales, frontal y el suelo, así como sus dimensiones y frecuencia característica, son recogidos en el apéndice $\mathrm{C}$, con el fin de no sobrecargar esta sección con detalles técnicos. Es necesario señalar que la pared en que se encuentra emplazada y enrasada la boca del escape es una pared 'infinita' perfectamente reflectante [124], con lo que se asegura que el sonido es radiado a un semiespacio esférico, eliminando así una posible indeterminación en el cálculo del ruido.

En definitiva, puede afirmarse que las medidas realizadas son adecuadas para la validación de los resultados obtenidos mediante el método de estimación experimental propuesto.

\subsubsection{Resultados de la validación}

La evaluación de los modelos de emisión se realiza mediante la comparación directa de la estimación del espectro de ruido radiado con los métodos propuestos a partir de las medidas de presión tomadas en el conducto, con su medida realizada con la sonda de intensidad en condiciones de campo libre. Este estudio se lleva a cabo para las tres posiciones consideradas del transductor de medida de presión en la cánula, de forma análoga a como se realizó en la evaluación de la estimación del caudal volumétrico en la cánula de escape, sección 2.4.1, con el objeto de analizar su influencia en el método de predicción. 
En primer lugar, se analizan en esta sección los resultados obtenidos con el modelo monopolar. Se debe aclarar a este respecto que para la obtención del nivel de presión sonora radiado mediante este modelo se emplea la ecuación (2.36) con la restricción adicional que conlleva la comparación con medidas de ruido realizadas en una cámara semianecoica, donde la superficie a la que se emite el ruido no es una esfera de radio $r$ sino una semiesfera de este mismo radio; por tanto, esta expresión quedará multiplicada por un factor 2 e incrementa en un factor $10 \log 2$ el espectro del nivel de intensidad sonora.

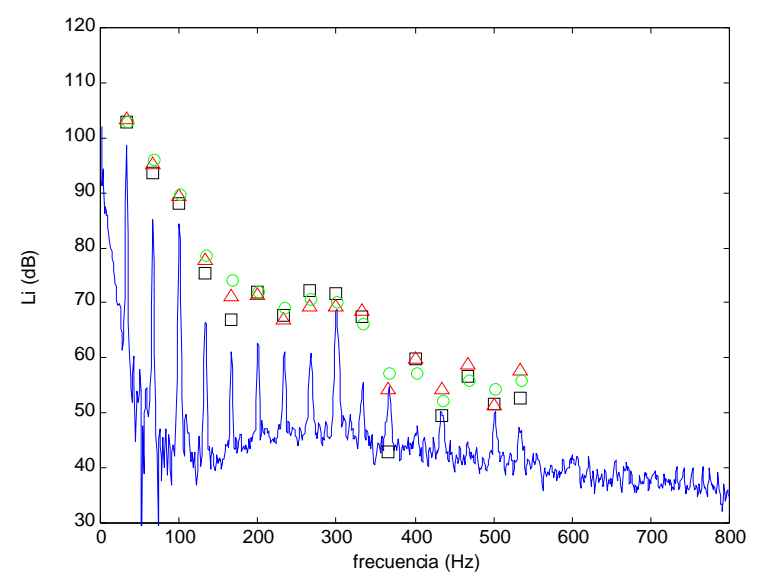

Figura 2.21: Ruido radiado a 1000 rpm: medido a $1 \mathrm{~m}$, $30^{\circ}$ (linea continua) y estimado mediante un modelo de emisión monopolar para la medida de presión en cánula a $0.1 \mathrm{~m}(\bigcirc), 0.15 \mathrm{~m}(\triangle)$ y $0.2 \mathrm{~m}(\square)$.

Los resultados de la predicción del espectro sonoro mediante la aplicación del modelo de emisión monopolar para los regímenes de giro 1000, 3000 y 6000 $\mathrm{rpm}$, se encuentran recogidos en las figuras $2.21,2.22$ y 2.23. En ellas se representa el ruido predicho frente a la medida exterior del mismo, observándose, fundamentalmente, dos aspectos; por un lado existe una sobrestimación global de las frecuencias de encendido del motor (armónicos), para un régimen de giro bajo hasta alcanzar H18, a partir del cual comienza a mejorar la estimación. Los niveles espectrales de los armónicos principales, son sobrestimados alcanzando diferencias en torno a $5 \mathrm{~dB}$ ó incluso $10 \mathrm{~dB}$ en algunos casos. Sin embargo, al aumentar el régimen de giro la situación es radicalmente distinta, obteniéndose ahora un buen acuerdo general entre los armónicos principales medidos y las estimaciones realizadas de los mismos, tanto a $3000 \mathrm{rpm}$ como a $6000 \mathrm{rpm}$. Esto muestra la capacidad del método para extraer información de las medidas de presión en el conducto, para regímenes de giro normales dentro 


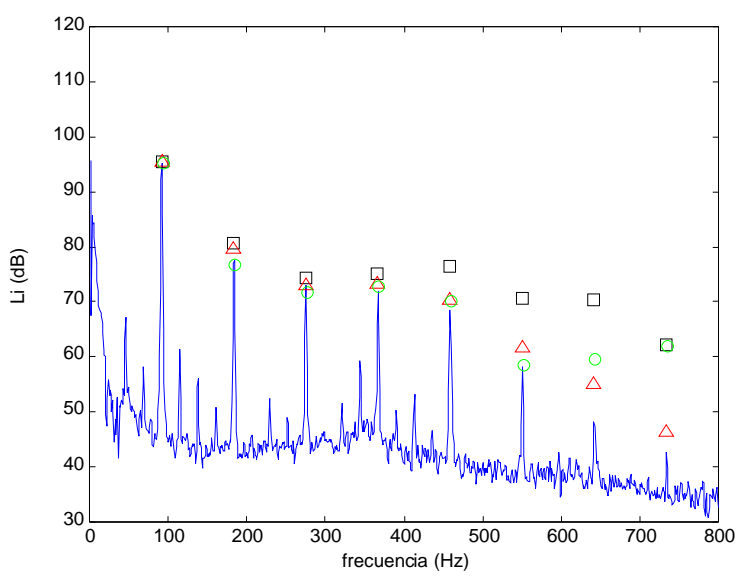

Figura 2.22: Ruido radiado a 3000 rpm: medido a $1 \mathrm{~m}$, $30^{\circ}$ (linea continua) y estimado mediante un modelo de emisión monopolar para la medida de presión en cánula a $0.1 \mathrm{~m}(\bigcirc), 0.15 \mathrm{~m}(\triangle)$ y $0.2 \mathrm{~m}(\square)$.

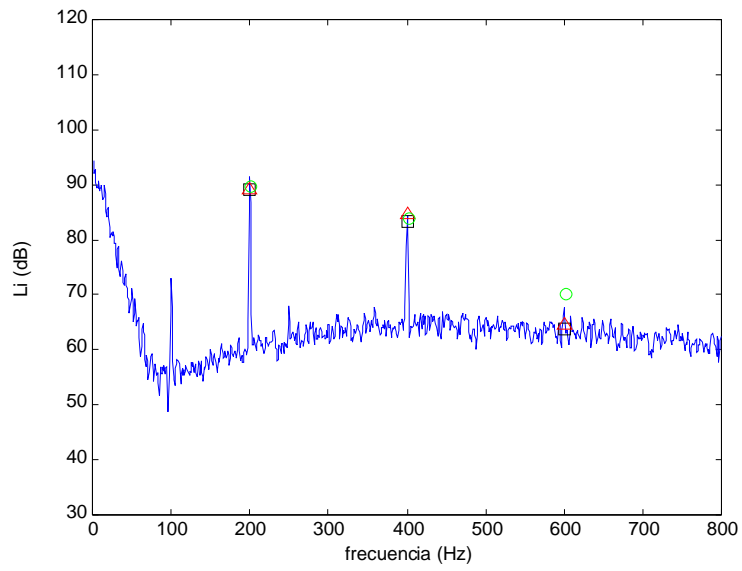

Figura 2.23: Ruido radiado a 6000 rpm: medido a $1 \mathrm{~m}$, $30^{\circ}$ (linea continua) y estimado mediante un modelo de emisión monopolar para la medida de presión en cánula a $0.1 \mathrm{~m}(\bigcirc), 0.15 \mathrm{~m}(\triangle)$ y $0.2 \mathrm{~m}(\square)$. 
del rango de funcionamiento de un motor. Además, se confirma la adecuación del modelo monopolar para reproducir la contribución de la componente pulsante del flujo emitido a excepción de regímenes de giro bajos, como ratifican los buenos acuerdos obtenidos a bajas frecuencias para el resto de regímenes de giro.

Otro rasgo importante que puede extraerse del ruido de escape medido a $1000 \mathrm{rpm}$, figura 2.21, es la importante excitación acústica observada en H18 $(300 \mathrm{~Hz})$; esto se debe al hecho de que a esta frecuencia se combinan dos efectos, uno debido a la componente pulsante (armónico del motor a este régimen de giro) y otro a la resonancia de la cámara excitada por el paso del flujo, como consecuencia de la cual se amplifica el armónico de motor a esta frecuencia.

En relación a la posición del transductor, los resultados previos indican que la mejor posición del transductor para la aplicación del procedimiento depende del régimen de giro del motor considerado, de modo que el transductor debe alejarse del extremo abierto a medida que el régimen de giro aumenta. Hecho que ya fue puesto de manifiesto en la sección 2.4.1. De este modo, podemos observar en la figura 2.21 como la mejor estimación a $1000 \mathrm{rpm}$ se obtiene para el registro de presión a $0.1 \mathrm{~m}$ del extremo abierto. Resultado esperable ya que la amplitud de la fluctuación de presión disminuye a medida que nos aproximamos al extremo abierto, como muestra la figura 2.12(a). Además, este punto está suficientemente próximo al extremo abierto y por ello las aproximaciones hechas para la propagación en el conducto poseen mayor validez. Al aumentar el régimen de giro del motor la influencia del ruido afecta más a las señales próximas a la boca de la cánula, y consecuentemente la mejor estimación a $3000 \mathrm{rpm}$ se obtiene a $0.15 \mathrm{~m}$ del extremo abierto, como se refleja en la figura 2.22. Finalmente al desplazarnos a altos regímenes de giro (6000 $\mathrm{rpm}$ ), los mejores resultados se obtienen para las medidas realizadas a $0.2 \mathrm{~m}$ del extremo de salida de la cánula, debido a que en ese punto se obtiene una mejor relación señal-ruido para altos regímenes de giro.

Esta dependencia de la posición de los transductores con el régimen de giro era esperable, ya que estas posiciones coinciden con aquéllas para las que se obtienen mejores estimaciones de la fluctuación del caudal volumétrico en la boca del escape a los mismos regímenes de giro, por lo que este resultado también se puede interpretar cómo una verificación de que es $\dot{\widetilde{u}}(t)$ la magnitud relacionada directamente con la generación interna, indicando de paso que es el modelo de emisión el factor que contiene los términos asociados a los procesos de descarga, como se había considerado al comienzo del trabajo.

Volviendo al modelo de emisión y a la vista de estos resultados resulta 
obvio que este modelo tan simple (monopolar) presenta ciertas limitaciones, tales como el hecho de que no contempla ningún factor de directividad. Factor que debe dar cuenta de las fuentes sonoras secundarias existentes alrededor de la boca de la cánula de escape, las cuales son forzadas por la interacción del flujo pulsante presente en dicha sección con la atmósfera en reposo. Estas fuentes son más relevantes a frecuencias superiores a unos $300 \mathrm{~Hz}$ tal como afirma Davies [46].

Por tanto, son presumiblemente esperables mejores resultados al considerar el carácter direccional del proceso de emisión. Las correlaciones entre el ruido medido y estimado con el procedimiento propuesto, derivadas de la consideración de que el extremo abierto radia como un pistón circular inserto en una pared rígida y su comparación con las estimaciones realizadas con el modelo monopolar, son mostradas en las figuras 2.24, 2.25 y 2.26. En ellas los espectros estimados con el modelo de emisión del pistón vibrante corresponden a la aplicación de la ecuación (2.50) a las medidas de presión realizadas en el interior de la cánula de escape, siendo estos transformados, posteriormente, a escala logarítmica y representados frente a las medidas de ruido exterior realizadas en condiciones semianecoicas de forma análoga a como se realizó en el caso anterior. Con el objetivo de no sobrecargar las gráficas y poder observar los efectos que introduce el factor de directividad con mayor claridad, se ha escogido el resultado de la estimación únicamente para la mejor posición del transductor hallada en cada caso, que se encuentra recogida en el pie de cada figura.

Se observa en las figuras de 2.24 a 2.26 que con este segundo modelo de emisión se reproducen resultados similares a los obtenidos con el modelo monopolar, con la salvedad de que el modelo de emisión del pistón vibrante proporciona unas mejores estimaciones a frecuencias superiores a los $300 \mathrm{~Hz}$, lo cual era esperable ya que a partir de estas frecuencias el efecto del flujo comienza a ser más relevante. A su vez, esta mejor estimación se preveía como respuesta al efecto, ya estudiado, de la eficiencia de radiación que reproduce este factor de directividad, en relación a su forma de decaimiento exponencial con la frecuencia, tendencia reproducida por las medidas de ruido exteriores.

A partir de los comentarios anteriores podríamos inferir las siguientes conclusiones:

- El método de cálculo presentado permite estimar el espectro del ruido de escape radiado, a partir de la presión medida en un punto próximo a la boca.

- En general el modelo de emisión de un pistón vibrante, que incluye un 


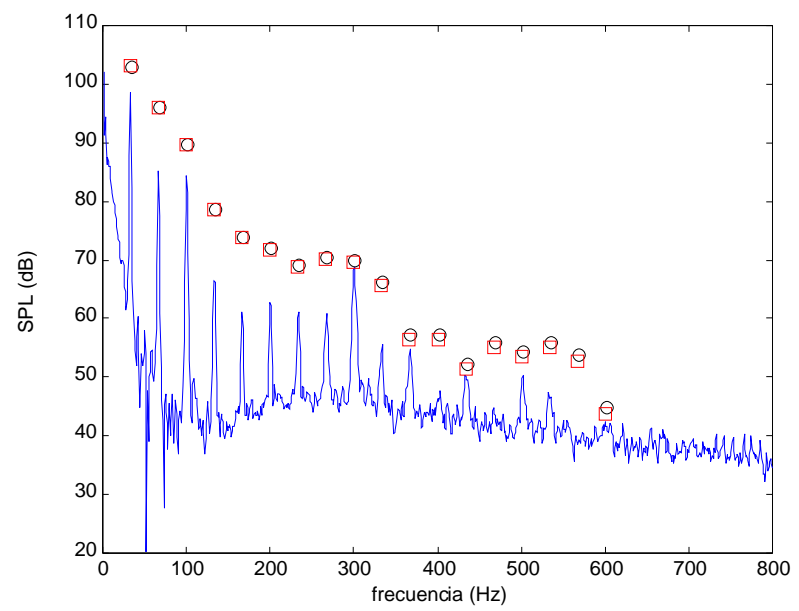

Figura 2.24: Ruido radiado a 1000 rpm: medido a $1 \mathrm{~m}$, 30 (linea continua) y estimado mediante la medida de presión en cánula a $0.1 \mathrm{~m}$ con el modelo de emisión monopolar (○) y con el modelo de emisión del pistón ( $\square)$.

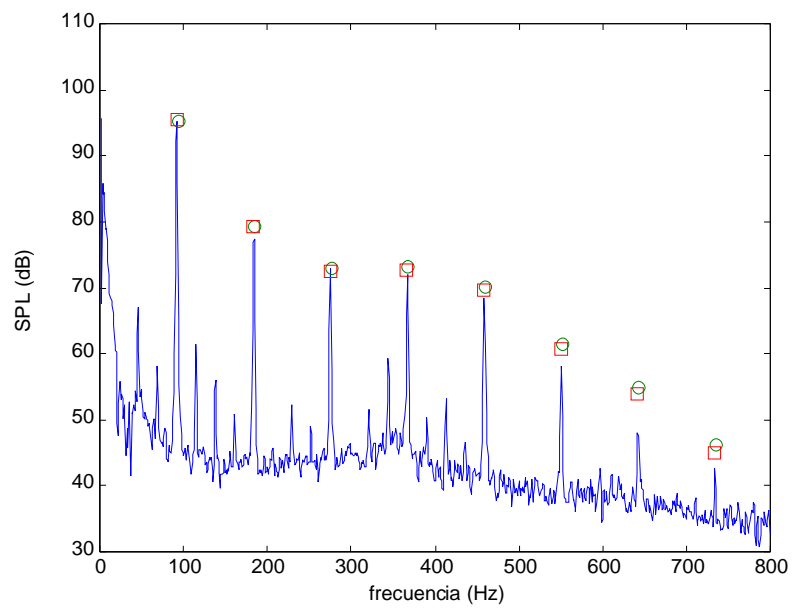

Figura 2.25: Ruido radiado a 3000 rpm: medido a $1 \mathrm{~m}$, $30^{\circ}$ (linea continua) y estimado mediante la medida de presión en cánula a $0.15 \mathrm{~m}$ con el modelo de emisión monopolar (○) y con el modelo de emisión del pistón ( $\square$ ). 


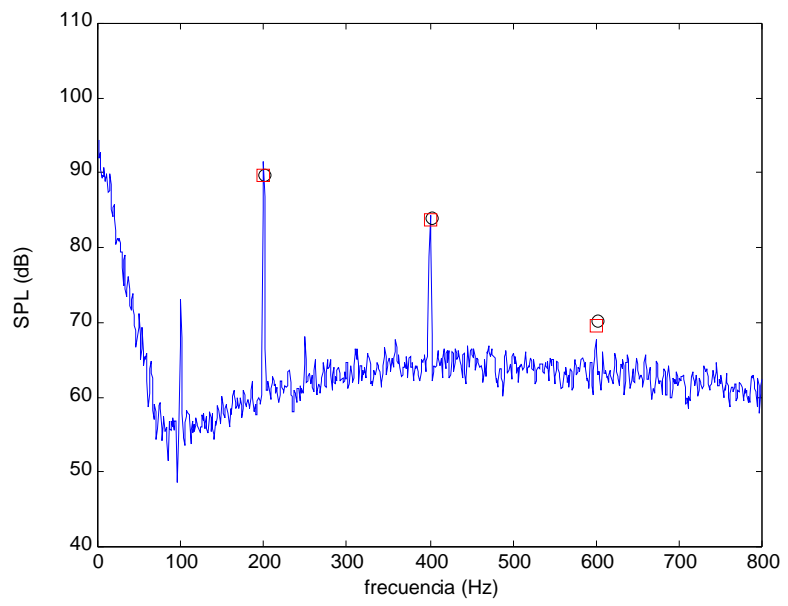

Figura 2.26: Ruido radiado a $6000 \mathrm{rpm}$ : medido a $1 \mathrm{~m}$, $30^{\circ}$ (linea continua) y estimado mediante la medida de presión en cánula a $0.2 \mathrm{~m}$ con el modelo de emisión monopolar (○) y con el modelo de emisión del pistón ( $\square)$.

factor de directividad de la fuente, trabaja mejor para cualquier rango de frecuencias. Si bien es cierto que estas diferencias frente al modelo monopolar son más evidentes para frecuencias superiores a unos $300 \mathrm{~Hz}$, hecho que esta relacionado con los efectos del flujo a estas frecuencias, en las cuales adquieren mayor importancia.

- Este método ofrece la ventaja de la posible estimación de las componentes armónicas del ruido de escape radiado en diferentes posiciones (distancias y ángulos), a través de una única medida de presión en el interior del conducto.

- Para la estimación a bajos regímenes de giro no se ha observado mejora apreciable para la sobrestimación obtenida para armónicos inferiores a H20 al introducir el factor de directividad de un pistón vibrante, hecho que explicaremos a continuación.

\subsection{Discusión de los resultados}

Se ha observado una sobrestimación general a bajos regímenes de giro entre las estimaciones y medidas realizadas del nivel de presión sonora, resultado que puede estar relacionado con el hecho de que el modelo de radiación 
aeroacústica empleado es válido sólo cuando la perturbación del flujo es muy pequeña en comparación con el flujo medio, como señalan Peizi et al. [136]. En este sentido, para analizar y cuantificar el límite de aplicabilidad del modelo de emisión del pistón vibrante a la predicción de las componentes armónicas del ruido de escape radiado, se estudia el valor medio del cociente entre la fluctuación de velocidad en el extremo abierto $(\widetilde{u}(t))$ y el valor medio de la velocidad del flujo $(U), \zeta=\langle\widetilde{u}(t) / U\rangle$. Valores representados en la figura 2.27 para las tres posiciones de medida de presión en la cánula de escape, comprobándose un colapso excelente entre las tres curvas. Además, en la evolución de $\zeta$, figura 2.27 , se observa un cambio brusco de este parámetro existiendo una evolución de decaimiento exponencial hasta regímenes de giro próximos a las $2000 \mathrm{rpm}$, trasladándose hacia un comportamiento de decaimiento prácticamente lineal a partir de este punto.

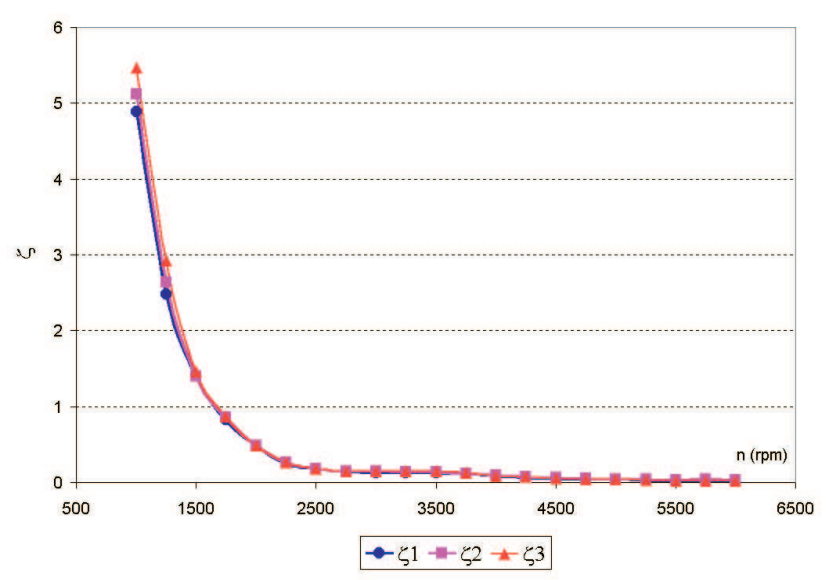

Figura 2.27: Representación del valor medio del cociente entre la fluctuación del caudal volúmetrico y el valor medio de la velocidad, $\zeta=\langle\widetilde{u}(t) / U\rangle$, frente al regimen de giro del motor para las tres posiciones de medida de presión: $\zeta_{1}$ a $0.2 \mathrm{~m}, \zeta_{2}$ a 0.15 $m$ y $\zeta_{3}$ a $0.1 \mathrm{~m}$.

A fin de verificar si este parámetro está realmente relacionado con el límite de validez del modelo de emisión se estudia a continuación el espectro de ruido estimado a $2000 \mathrm{rpm}$ empleando el modelo de emisión del pistón vibrante, ver figura 2.28, pudiéndose observar un buen acuerdo entre los resultados de la estimación y las medidas de ruido, lo que indica que el factor $\zeta$ escogido es adecuado para analizar el comportamiento de la emisión. La información que se extrae de él es la de la dependencia del cociente $\zeta=\langle\widetilde{u}(t) / U\rangle$ 


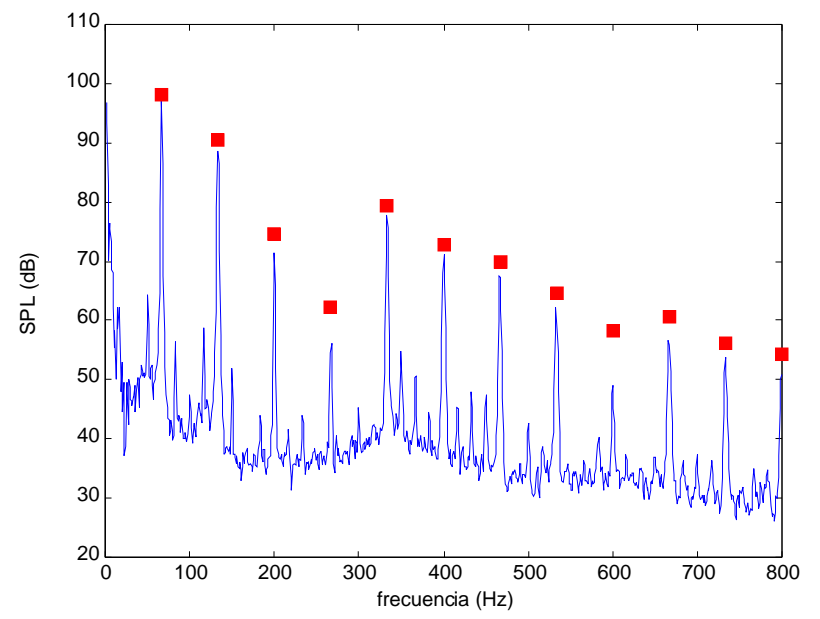

Figura 2.28: Ruido radiado a 2000 rpm: medido a $1 \mathrm{~m}$, $30^{\circ}$ (linea solida) y estimado para la medida de presión a 0.15 m con el modelo de emisión del pistón vibrante

con el límite de validez del procedimiento para la estimación de la energía acústica radiada para valores de $\zeta$ por debajo de 0.5. Esta relación supone una sobrestimación de la presión sonora radiada para altas amplitudes de la componente pulsante en comparación con el flujo medio, donde la influencia del comportamiento no lineal del extremo de un conducto sobre las características de reflexión adquiere mayor importancia, existiendo además una mayor absorción de la energía acústica por parte de la capa vortical desarrollada en dicho extremo. Afirmación que corroboran Peters et al. [137], añadiendo que el efecto no lineal más importante observado en el extremo de un conducto, es el asociado con la separación no estacionaria del flujo acústico y la formación del tren de vórtices ordenados que conlleva. Este fenómeno ocurre cuando la amplitud del desplazamiento acústico es mayor que el radio del conducto, es decir, para condiciones en las que el número de Strouhal acústico, $S t_{a c}=\omega \cdot a /$ $\langle\widetilde{u}(t)\rangle$, es pequeño $\left(S t_{a c}<1\right)$. Este comportamiento no lineal supone un fuerte incremento en la absorción del sonido debido a la conversión de energía acústica en energía cinética de los vórtices.

Esta explicación es coherente con los resultados experimentales obtenidos, ya que por un lado, como puede verse en la figura 2.29, para pequeños números de Strouhal acústicos se obtiene una clara sobrestimación en los niveles predichos, debido a que en el modelo de predicción no se han incluido la energía acústica absorbida por los vórtices. Sin embargo, al aumentar el número de Strouhal $\left(S t_{a c}>1\right)$ las predicciones mejoran. 


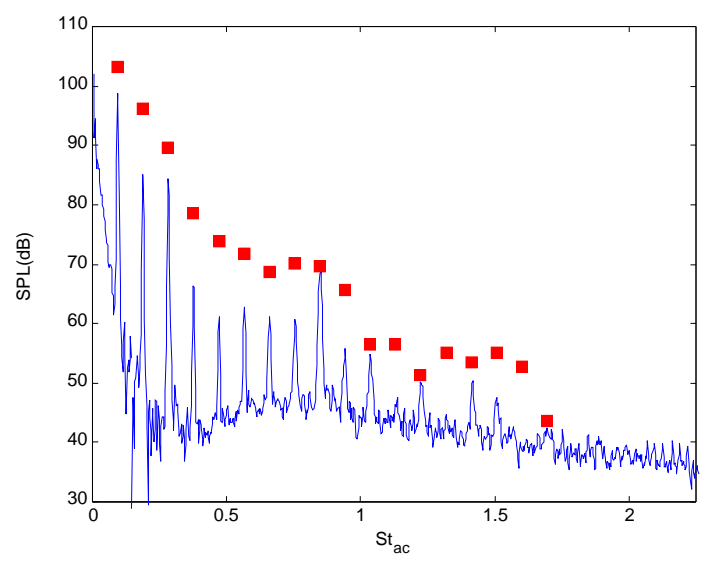

Figura 2.29: Espectro de ruido radiado a 1000 rpm: medido (linea continua) a 1m, $30^{\circ}$, y estimado desde la medida de presión en cánula a $0.1 \mathrm{~m}$ del extremo (ם) como una función del número de Strouhal acústico.

Por otro lado, como muestra la figura 2.30, los efectos de no linealidad en el sistema considerado son más importantes para valores de $\zeta$ superiores a 0.5 , para los que los números de Strouhal acústicos correspondientes a la frecuencia del primer armónico del motor (H2) es inferior a la unidad. Estos valores del parámetro $\zeta$ para la línea de escape y motor considerados corresponden a bajos regímenes de giro como se comprueba en la figura 2.27. Si además tenemos en cuenta la presencia de efectos de reflujo a estos regímenes de giro, parece aún más razonable la sobrestimación obtenida con el modelo de emisión del pistón, donde tampoco está incluido este factor de absorción de la energía radiada.

El efecto del reflujo puede observarse con mayor claridad en la figura 2.31, donde se representan los resultados para un cálculo CFD llevado a cabo con el código comercial FLUENT. En esta figura se observa que cuando existe reflujo, ver gráfico de la izquierda, el flujo saliente que provenía desde el silenciador captura parte de la energía de los vórtices exteriores incrementando la energía cinética (velocidades más elevadas) de los vórtices internos, y cómo consecuencia de esta conversión de energía cinética disminuye la velocidad de los vórtices exteriores, asociados al flujo emitido. Este último comentario se ratifica en la parte derecha de la figura 2.31, donde puede verse como el flujo que vuelve a entrar en la cánula es alimentado por el vórtice exterior empleando la energía de este último para incrementar la suya.

El cálculo realizado considera efectos débilmente compresibles, de acuer- 


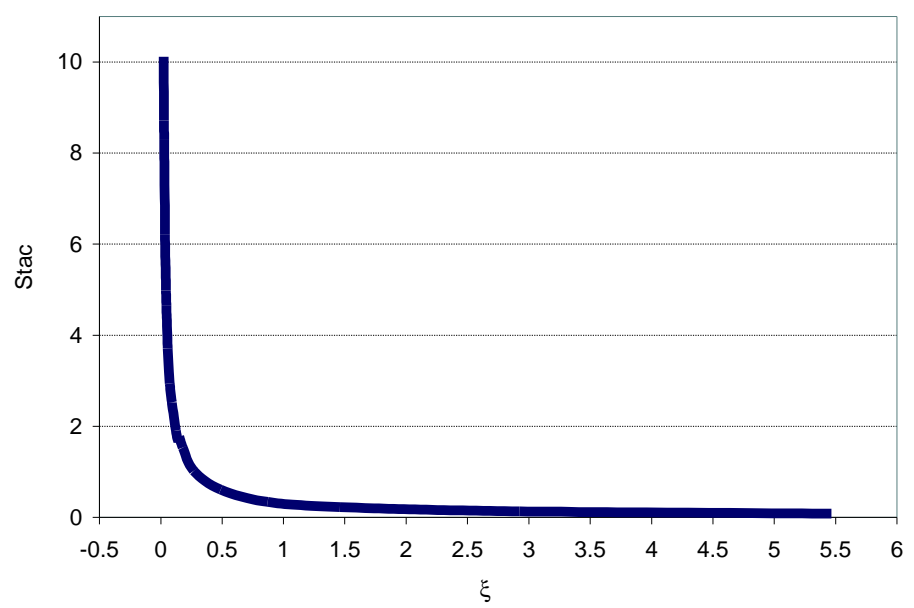

Figura 2.30: Evolución del número de Strouhal acústico para la frecuencia carácteristica de la señal, $f_{c}=4 \cdot n / 120$, con el parámetro $\zeta$.
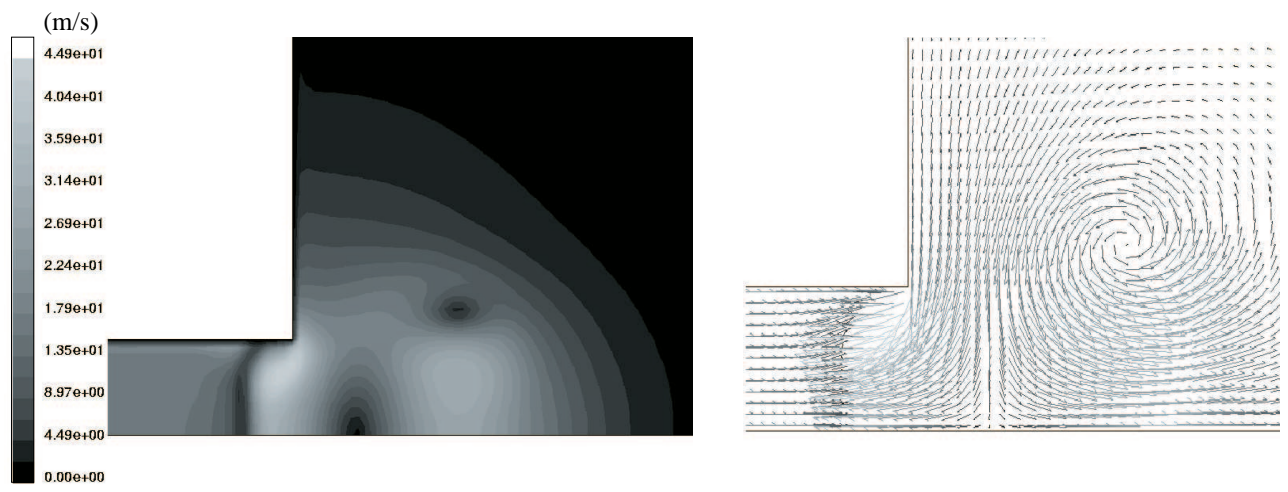

Figura 2.31: Contornos de la magnitud de la velocidad y los vectores velocidad en la sección de salida del escape cuando existe reflujo del flujo de escape saliente para la fluctuación de la velocidad a 1000 rpm.

do con el rango del número de Mach de interés, y se modela la turbulencia mediante un modelo $\mathrm{k}-\varepsilon$ convencional. En esta aproximación la fluctuación de velocidad del flujo se emplea como una condición de contorno de entrada en un dominio bidimensional axisimétrico, con una cánula de entrada de 4.0 $m$ de longitud, que desemboca en una cámara de $2.0 \times 2.0 \mathrm{~m}^{2}$ que posee condiciones de contorno de campo libre en sus extremos a fin de modelar el volumen de emisión. La longitud de la cánula se escoge de $4 \mathrm{~m}$ con el objetivo 
de evitar que las reflexiones que puedan producirse en el extremo abierto de la entrada de la misma puedan perturbar el pulso emitido. De la misma forma la condición de campo libre en los lados de la cámara, que constituye el dominio de emisión, aseguran las mismas condiciones que en la cámara semianecoica. La fluctuación de velocidad del flujo se obtiene a través de la estimación de la fluctuación de la velocidad en el extremo abierto, utilizando las ecuaciones (2.9) y (2.10), pero propagando ahora la fluctuación de la velocidad desde el extremo abierto hasta un punto situado a $4.0 \mathrm{~m}$ en el interior de la cánula de escape.

Se puede concluir diciendo que de forma general el método propuesto permite estimar el espectro de ruido de escape radiado con un grado de precisión adecuado (errores de $\pm 1 \mathrm{~dB}$ ) para valores de $\zeta$ inferiores o iguales a 0.5 , ofreciendo la ventaja de poder obtener este espectro a diferentes distancias y posiciones angulares partiendo de una única medida de presión en la cánula de escape, lo que proporciona una disminución importante del coste experimental, al no ser necesaria una enorme colección de medidas exteriores para conocer el espectro radiado en diversas posiciones. Además, permite identificar los mecanismos de generación sonora al separar la contribución de la fuente interna, elementos de la línea de escape que se reflejan en la estimación de la derivada del caudal volumétrico en la sección de salida de ésta, y las fuentes externas resultantes de la interacción de esta aceleración de volumen con la atmósfera en reposo. De este modo, la sobrestimación que se realiza para valores de $\zeta$ mayores que 0.5 (bajos regímenes de giro), se debe a los efectos de no linealidad y reflujo que se producen en estos casos y que conllevan una mayor absorción de energía cinética por parte del movimiento vortical que aparece en torno a la boca de escape, conversión de energía o factores de absorción que el modelo de emisión no contempla.

\subsection{Carácter estacionario del ruido de flujo en es- capes}

Para el análisis del ruido de flujo emitido en el proceso de escape, hemos de tener en cuenta que la generación de ruido de flujo está asociada con el establecimiento de patrones de flujo no estacionario, los cuales pueden transferir una cierta cantidad de su energía al campo acústico. En el caso de flujos predominantemente 'estacionarios', las componentes no estacionarias están directamente relacionados con las inestabilidades del flujo y la turbulencia (es una experiencia habitual que los flujos laminares no producen sonido [52]). El problema de generación de ruido de flujo en un motor de combustión interna 
es, en principio, más complejo, debido al carácter intrínsecamente no estacionario del flujo, impuesto por el comportamiento cuasiperiódico del motor en sí mismo.

Debido a la complejidad del fenómeno de escape (componente pulsante y ruido de flujo) es prácticamente imposible el tratar de analizar el problema completo. Afortunadamente, las emisiones de ruido de flujo se convierten en las contribuciones predominantes del ruido de escape sólo a muy altos regímenes de giro, a los cuales las cantidades de gases de escape emitidas por unidad de tiempo desde el motor son mayores, de modo que la velocidad media en la boca de escape es cuanto menos del mismo orden de magnitud que las fluctuaciones de velocidad asociadas al flujo pulsante; y cuyos efectos son más importantes para frecuencias superiores a $300 \mathrm{~Hz}$ a cualquier régimen de giro [46]. Bajo estas condiciones es razonable considerar que el flujo medio y las fluctuaciones de flujo asociadas con el proceso de renovación de la carga a frecuencias bajas y medias están desacopladas hasta cierto punto, de forma que se puede considerar el flujo como la superposición de un flujo medio 'estacionario', responsable de la generación del ruido de flujo, y un flujo pulsante responsable de las características 'armónicas' del ruido de escape. Esto puede, apreciarse más claramente al comparar los espectros de ruido obtenidos a diferentes regímenes de giro de motor (velocidades de flujo), gráfico de la derecha de la figura 2.32, y los obtenidos en la descarga estacionaria de un flujo por una cánula del mismo diámetro de salida, gráfico de la izquierda de la figura 2.32. Del análisis de estas figuras se extrae que la forma de los "plateaus" y la tendencia de crecimiento al aumentar la velocidad del flujo en el caso de la emisión en un motor es claramente reproducida por la descarga de un flujo estacionario, para el mismo rango de números de Mach. El hecho de incluir, en ambas gráficas, una escala frecuencial muy diferente (ver figura 2.32) se debe a que, como ya se mencionó en el capítulo de introducción, mientras que la componente pulsante del ruido de escape tiene una contribución más importante a bajas frecuencias el ruido de flujo la tiene a frecuencias mayores a partir de $300 \mathrm{~Hz}$. Sin embargo, si observamos la gráfica de la derecha (espectro del motor) el haber considerado mayores frecuencias (a partir de 500 $\mathrm{Hz}$ ) hubiera aproximado más aún el comportamiento de ambas gráficas.

Además, como se aprecia en la figura 2.33, con el modelo de estimación de la componente pulsante del ruido de escape, presentado en este capítulo se reproduce el $99 \%$ de los armónicos con una precisión de $\pm 1 \mathrm{~dB}$, poniéndose de manifiesto que la componente armónica, del espectro del ruido de escape, no está aparentemente afectada por el efecto del flujo medio. De modo, que parece coherente el asimilar el efecto del ruido de flujo únicamente a un comportamiento estacionario de emisión, como muestra las tendencias observadas 

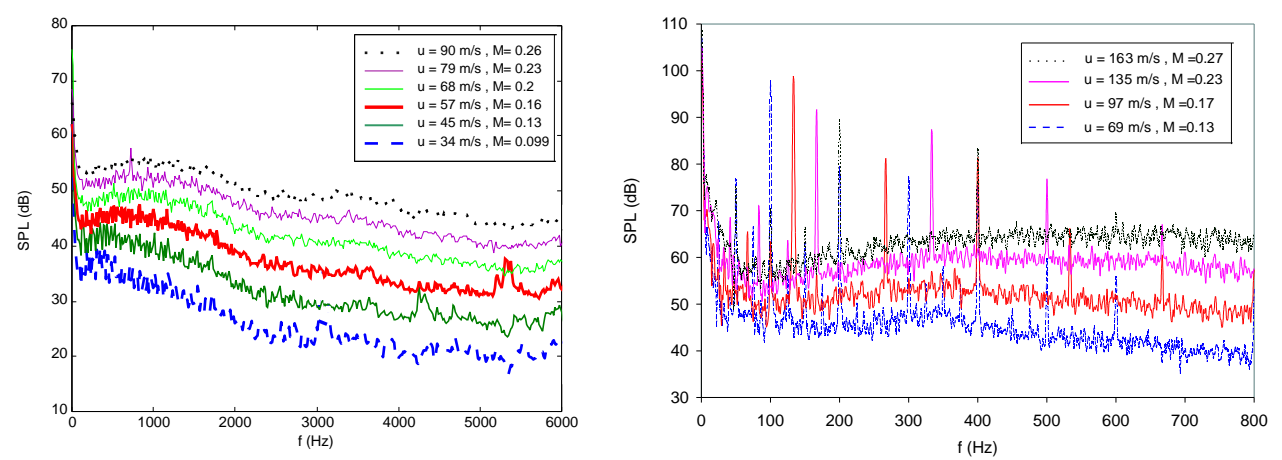

Figura 2.32: Representación del espectro de ruido radiado por la descarga de un flujo estacionario en un tubo recto (gráfico de la izquierda), y por el escape de un motor a diferentes regímenes de giro (gráfico de la derecha).

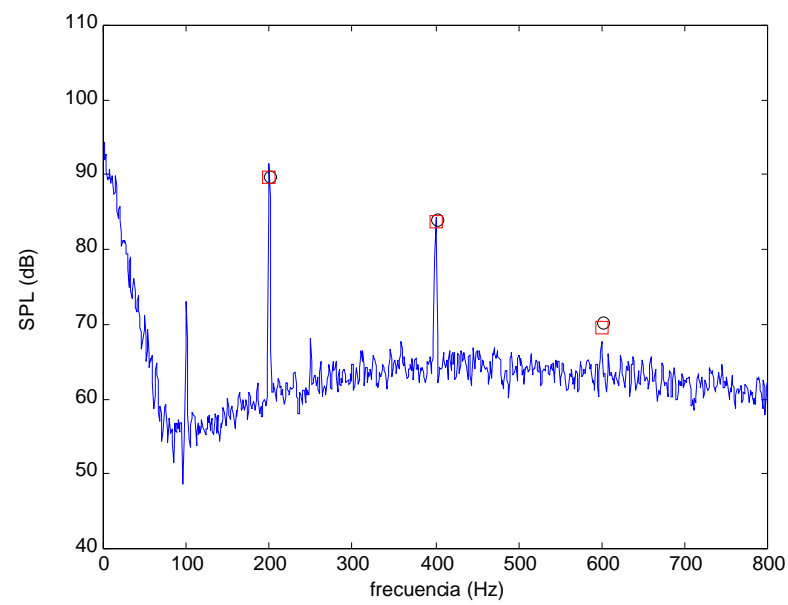

Figura 2.33: Ruido radiado a 6000 rpm: medido (linea continua) a $1 \mathrm{~m}$, $30^{\circ}$ y estimado mediante para la medida de presión en cánula a $0.2 \mathrm{~m}$ con el modelo de emisión monopolar (○) y con el modelo de emisión del pistón ( $\square$ ). 
en la figura 2.32, si prescindimos de la contribución armónica en el espectro del ruido de escape radiado (gráfica de la derecha).

Todo esto demuestra que es posible realizar un estudio separado de los dos fenómenos (ruido de flujo y pulsante), de por sí suficientemente complejos, a pesar de que finalmente, en un chorro pulsante, la consideración de interacciones mutuas de ambas contribuciones consideradas pueda ser necesaria cuando los efectos de no linealidad son más importantes $(\zeta>0.5)$, como indican los resultados mostrados por Peizi y Genhua [135] y comentados en este capítulo. De este modo, en el capítulo siguiente y de acuerdo con las ideas defendidas por Desantes et al. [52], se considerará que la generación de ruido de flujo en el sistema de escape es esencialmente debida a la existencia de un flujo medio estacionario, en el cual se desarrolla un patrón de flujo localmente no estacionario, debido a una turbulencia completamente desarrollada y a las inestabilidades del flujo (como aquellas originadas en las capas de mezcla y cizalladura). 


\section{Capítulo 3}

\section{Estudio experimental del ruido de flujo estacionario}

3.1 Introducción . . . . . . . . . . . . 81

3.2 Descripción del método de intensimetría en conductos ................... 82

3.2.1 Montaje experimental . . . . . . . . . . . 84

3.2.2 Método de cálculo de la intensidad radiada . . . . 88

3.3 Teoría de la descomposición de ondas . . . . . . 91

3.3.1 Método de los dos micrófonos . . . . . . . . . . . 92

3.3.2 Descomposición de ondas mediante técnicas de conformación del haz. . . . . . . . . . . . . . . . 94

3.3.3 Conformador del haz de mínima varianza con restricciones lineales (LCMV) . . . . . . . . . 100

3.3.4 Consistencia del método de descomposición . . . . 102

3.4 Validación de la potencia de la técnica de estimación de la intensidad . . . . . . . . . . . 103

3.4.1 Análisis del proceso de emisión . . . . . . . . . . 108 



\subsection{Introducción}

De acuerdo con el planteamiento presentado en el capítulo anterior se asume un cierto desacoplamiento entre la generación del ruido del flujo medio por el sistema de escape y la contribución del ruido pulsante, desacoplamiento válido a regímenes de giro altos, a los cuales el gasto trasegado por el motor es mayor, de modo que la velocidad media en la boca de escape es cuanto menos del mismo orden de magnitud que las fluctuaciones de velocidad asociadas al flujo pulsante. Pudiéndose considerar el flujo como la superposición de un flujo medio 'estacionario', responsable de la generación del ruido de flujo, y un flujo pulsante responsable de las características 'armónicas' del ruido de escape. En este capítulo nos centraremos en la generación del ruido producido por un flujo estacionario, considerando que reproduce el comportamiento del ruido de flujo en el motor de forma adecuada, en las condiciones mencionadas arriba.

Sabemos que un sistema de escape consiste esencialmente en un conjunto de silenciadores y elementos conectados por una secuencia de conductos de secciones uniformes. Las ondas reflejadas en las uniones, combinadas con la generación de sonido local producen un campo acústico que comprende a las ondas estacionarias y progresivas [51]; a éstas se les superpone en el tiempo un flujo medio, incluyendo este movimiento de fluido inestable tanto la acústica relacionada con las fluctuaciones de presión y velocidad como su combinación con las perturbaciones vorticales emplazadas en las capas de corte o generadas en los contornos, que viajan con el flujo medio.

Por todo ello es interesante plantear una nueva técnica de medida que permita evaluar la generación de ruido de flujo por las componentes individuales del sistema de escape. La técnica escogida, que se presenta y discute en este capítulo, es la de intensimetría en conductos, que en la versión propuesta aquí utiliza los registros de presión adquiridos por cuatro micrófonos emplazados en la cánula de salida para condiciones similares a las de funcionamiento del motor [169], a partir de los cuales, empleando un algoritmo de descomposición basado en la técnica del 'beamforming', se extraen las componentes incidente y reflejada de las ondas de presión en la cánula de escape, componentes que permitirán estimar la intensidad transmitida.

Los ensayos se llevan a cabo en un banco de flujo frío con un flujo de velocidad media constante, con el que es posible simular las condiciones de flujo de un motor [92]. La viabilidad del uso de un flujo frío para reproducir condiciones de motor en las que el flujo es caliente y por tanto posee otras propiedades, se debe a que en el caso del ruido de flujo es el número de Mach 
el parámetro que parece ser apropiado para correlacionarlo. De forma que si el gasto másico y la temperatura son condiciones conocidas, el nivel sonoro puede extrapolarse a condiciones reales, siempre que los rangos de números de Mach empleados sean similares a los obtenidos en un motor.

Esta técnica permite dar respuesta a las necesidades de la industria de poseer valoraciones experimentales sobre las emisiones sonoras tanto del sistema de escape completo como de sus componentes [172], y de los modelos predictivos existentes, asegurando así un desarrollo del sistema de escape más rápido y de menor coste. Además el sistema de escape, como se ha mencionado, está formado por un conjunto de elementos simples como cámaras (expansiones y contracciones), tubos de conexión, curvaturas, etc., y complejos (perforados, etc.), los cuales interaccionan con el flujo constituyéndose como fuentes generadoras de esta componente sonora (ruido de flujo). Las medidas tradicionales realizadas sobre el ruido emitido por la cánula de escape no proporcionan suficiente información para indicar los cambios concretos que se requerirían de cada elemento para optimizar el sistema de escape completo, ni para identificar las fuentes de ruido de flujo distribuidas a lo largo del sistema de escape. En efecto, experimentalmente es imposible estimar la contribución relativa de cada mecanismo de ruido a través únicamente de las medidas realizadas en campo libre, por incluir éstas tanto la contribución del ruido de flujo generado internamente como por fuentes externas tales como el generado en la boca de salida (sonido de los vórtices), e incluso el ruido causado por la reflexión en un plano o en otros objetos cercanos.

Por tanto, este procedimiento experimental de estimación del ruido de flujo permitirá obtener información importante sobre el ruido generado por los elementos aislados del sistema de escape, en función de los parámetros del flujo, velocidad media y temperatura.

\subsection{Descripción del método de intensimetría en con- ductos}

Como se ha mencionado, en este capítulo se presenta un técnica experimental para evaluar el ruido de flujo generado por los elementos aislados del sistema de escape. En particular, nos centraremos en el análisis del ruido de flujo generado por los silenciadores.

La generación de ruido de flujo está asociada al establecimiento de patrones de flujo no estacionario, los cuales pueden transferir una cierta cantidad de su energía al campo acústico [52]. En el caso de flujos que se consideran 
estacionarios en términos de las variables medias del flujo, estos patrones de flujos no estacionarios están directamente relacionados con las inestabilidades del flujo y la turbulencia (es una experiencia habitual que los flujos laminares estables no producen sonido).

Sin embargo, la comprensión de la turbulencia es todavía insuficiente; históricamente el desarrollo que ésta ha sufrido puede resumirse del siguiente modo. En los años 50 la turbulencia se consideraba constituida por un conjunto de pequeños remolinos; como resultado, dentro de la investigación de ruido de flujo la atención recayó sobre la cuantificación del ruido asociado a las pequeñas escalas turbulentas, línea de trabajo que persistió hasta los 80 . Sin embargo, el descubrimiento de amplias estructuras turbulentas en la capa límite del flujo a principio de los 70 condujo a muchos investigadores a cuestionarse la validez de estas hipótesis y al establecimiento de nuevas teorías que sugerían que, para altas velocidades de los flujos, eran las amplias estructuras turbulentas (ondas de inestabilidad del flujo) las responsables de la parte dominante del ruido de mezcla del chorro. Por otro lado, a partir de los años 80 comenzó a disponerse de instrumentación para la realización de medidas específicas de ruido de flujo, lo que permitió acumular una gran cantidad de datos en una amplia banda de velocidades de flujo. Un análisis reciente de estos datos [163] ha suministrado una irrefutable evidencia de que el ruido de flujo está constituido por dos componentes básicas: una asociada a las grandes estructuras turbulentas y otra a las finas escalas turbulentas, afirmación válida incluso para flujos subsónicos.

Por tanto, debido a las incertidumbres existentes en el tratamiento de la turbulencia y a pesar de que el problema de generación del ruido de flujo en un motor de combustión interna es en principio más complejo, debido al carácter intrínsecamente no estacionario del flujo impuesto por el comportamiento cuasiperiódico del motor en sí mismo, como ya se justificó en el capítulo anterior, es razonable asumir bajo ciertas circunstancias, que la generación de ruido de flujo en el sistema de escape se debe esencialmente a la existencia de un flujo medio estacionario, en el cual un patrón de flujo localmente no estacionario se desarrolla debido a una turbulencia completamente desarrollada y a las inestabilidades del flujo (como aquéllas originadas en las capas de mezcla y cizalladura) [52].

Aunque la mayoría de los mecanismos físicos han sido comprendidos, la realización de predicciones cuantitativas se topa con el hecho de que además del conocimiento de la fuente se debe observar el modelo de emisión sonora en su escala completa [43]. En sistemas con geometrías complejas, tales como los de admisión y escape, la identificación de estas fuentes de excitación acústica 
inducidas por el flujo es importante, ya que pueden afectar a las medidas en conducto presentando varios problemas en la separación de las fluctuaciones de presión o el ruido de flujo procedente de contribuciones más coherentes generalmente asociadas al movimiento de ondas acústicas o al flujo inducido por la dinámica del flujo turbulento [75]. En respuesta a este problema, el primer objetivo de la técnica de medida presentada es reproducir estimaciones fiables de la potencia sonora del flujo coherente en cualquier punto del sistema donde se sospecha que existen fuentes de ruido de flujo. En particular, se escoge la medida del efecto de su conjunto en la cánula de escape, ya que la idea que subyace de la medida con micrófonos en la cánula de salida es la de poder medir la región fuente.

\subsubsection{Montaje experimental}

Esta nueva técnica de medida, como ya ha sido mencionado, se basa en el registro de presión en la cánula de escape, procedimiento similar al de medida en motor (presentado en el capítulo anterior); sin embargo, la metodología desarrollada y el banco de pruebas empleado es completamente diferente al presentado en aquel capítulo, difiriendo por tanto también el tipo de medidas realizadas.

La descripción del dispositivo experimental empleado se dividirá en dos partes. La primera se refiere al sistema generador de flujo, excitación en el elemento a ensayar y su transporte hasta el elemento bajo ensayo, el silenciador, y la segunda al sistema de adquisición de señales de presión instantánea, que constituyen la base de nuestro procedimiento de estimación.

La generación de una corriente fluida en condiciones estacionarias requiere la definición de una instalación en la que podamos controlar la cantidad de aire trasegada a través de un conducto, minimizando el efecto de las fluctuaciones originadas por el funcionamiento del propio generador de flujo. Esta instalación es la que denominamos Banco de Flujo frío, la cual se encuentra representada en la figura 3.1. El Banco de Flujo está constituido por una línea alimentada por un compresor volumétrico de tres lóbulos rotativos que constituye el elemento generador del flujo estacionario. Este flujo se hará pasar a través del silenciador, elemento a ensayar, para medir mediante una serie de captadores (micrófonos) enrasados en la pared de la cánula de salida, la fluctuación de presión producida por el flujo al atravesar los distintos elementos que componen la geometría del silenciador. Simultáneamente, en la cámara semianecoica, se medirá el ruido producido a la salida de la cánula, procedimiento que no será detallado en esta sección ya que fue abordado con suficiente amplitud en el capítulo anterior. 


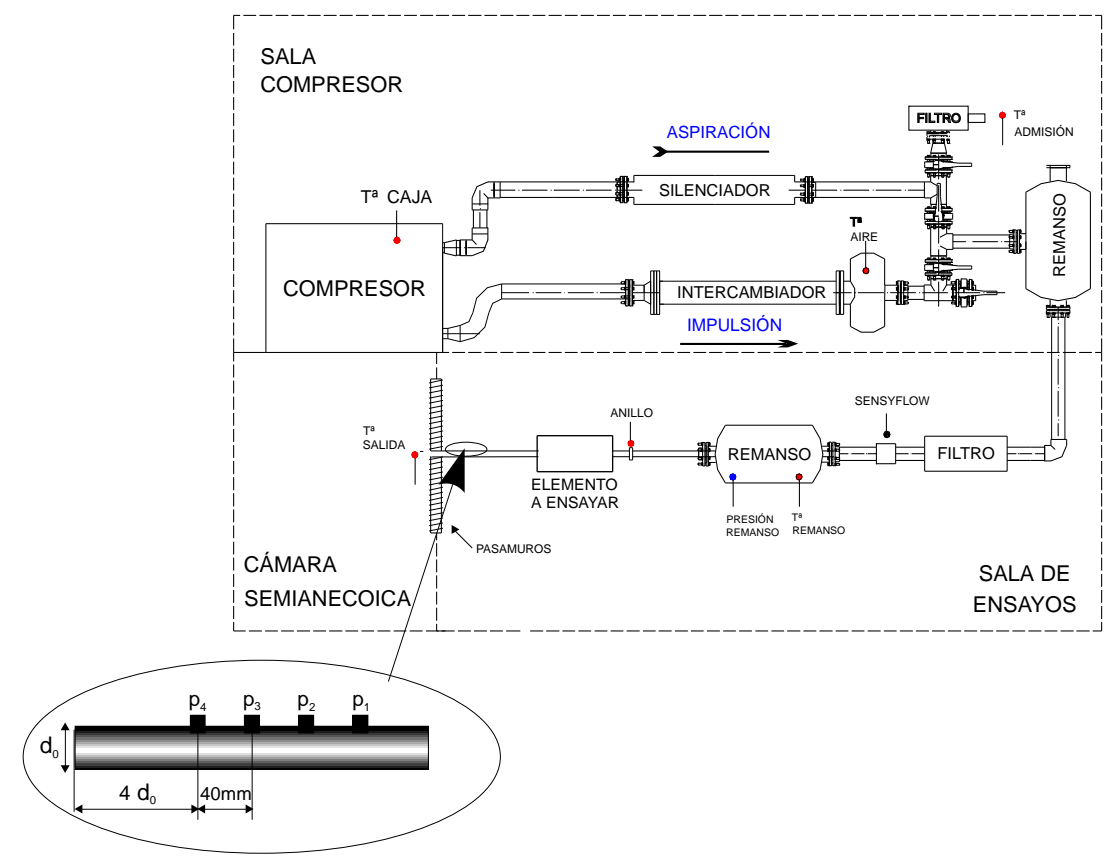

Figura 3.1: Elementos del dispositivo de medida empleado en la validación de la técnica de intensimetría en conductos.

El generador del flujo se encuentra en una sala separada del resto de la instalación, situada en una planta superior, debido al elevado nivel de ruido que genera su funcionamiento y que podría perfectamente contaminar las medidas que se lleven a cabo. En esta misma sala se encuentran también el motor eléctrico que lo acciona, junto con las válvulas electroneumáticas de control, las válvulas de mariposa y el variador de frecuencia, con todo lo cual se puede controlar con precisión el gasto que transita por el cuerpo del silenciador. El gasto medio del flujo generado se mide empleando un medidor de hilo caliente, "sensyflow", medida por tanto de alta calidad debido a la sensibilidad de este sistema. La calidad de esta medida es importante ya que será ella junto con la medida de la temperatura las que determinarán la velocidad media del flujo que transita por el elemento ensayado, parámetro fundamental en el conocimiento del ruido de flujo generado por este sistema.

El funcionamiento de está instalación, ver figura 3.1, puede resumirse como sigue: en primer lugar el compresor aspira aire del exterior e impulsa el flujo hacia un primer depósito (remanso), donde es estabilizado, eliminando así las fluctuaciones del flujo; mediante una serie de conductos se lleva hasta la sala 
donde se encuentra el elemento a caracterizar (asegurando que el flujo incidente no esté afectado por ninguna perturbación procedente de la instalación del banco de flujo antes descrita), y una vez en esta sala, una válvula de mariposa manual deja paso al flujo estacionario. Éste se hace circular por un gran filtro antes de atravesar el medidor de hilo caliente (medidor de gasto), a fin de eliminar las posibles impurezas del aire que pudiesen afectar a la medida del gasto medio, ya que el medidor de hilo caliente es muy sensible al polvo. Posteriormente el flujo es transportado hasta un segundo remanso situado en la sala de ensayos, donde el aire se estabiliza garantizando condiciones de parada del mismo para la medida de presión estática y temperatura. Finalmente, el flujo desemboca en el silenciador a través de un tubo cuya longitud ha de ser como mínimo 10 veces el diámetro de la cánula de entrada para asegurar que el flujo está estabilizado y evitar el ruido generado por la posible turbulencia presente en el cambio de sección (del remanso al tubo). Al igual que en el caso de las medidas en motor, la boca de la cánula de escape de dicho silenciador se encuentra enrasada con la pared que comunica dicha sala de ensayos con la cámara semianecoica, debido a las ventajas que esto supone (mencionadas en el capítulo anterior).

El montaje de los micrófonos en el conducto de salida del silenciador es quizás una de las partes más delicadas del dispositivo de medida. Este montaje debe cumplir unos requisitos que se describen a continuación.

En primer lugar se sitúan los cuatro micrófonos en la cánula de salida de forma que la posición del primer micrófono se encuentre a una distancia de doce diámetros de la entrada de esta cánula [48], a fin de asegurar la hipótesis de flujo turbulento totalmente desarrollado en los puntos de medida ${ }^{1}$, y el último micrófono a 4 diámetros del extremo abierto de la cánula con el objeto de que su medida no se vea afectada por los efectos de la descarga del flujo y por tanto contenga información únicamente de la fuente interna. En segundo lugar, la distancia entre los micrófonos se fija en $40 \mathrm{~mm}$, como compromiso entre los errores de medida a baja frecuencia $[1,110]$ y el criterio de Nyquist para evitar el fenómeno de "aliasing" espacial al que se hará referencia en la sección 3.3.2.

Con estos micrófonos se realizan registros de presión de $2 \mathrm{~s}$ de duración con la finalidad de disponer de una medida de presión fiable y representativa del proceso real, permitiendo identificar así posibles efectos de no estacionariedad en la señal.

\footnotetext{
${ }^{1}$ Debe recordarse que puesto que las cánulas de salida poseen longitud finita, aunque sea grande, cualquier perturbación puede transportarse fuera de la cánula antes de que llegue a romper el flujo laminar [95].
} 
Además de esta configuración de los micrófonos que denominamos disposición en línea y que sirve de base para la posterior descomposición de las señales de presión en sus componentes incidente y reflejada, se escoge otra disposición de los mismos que denominamos en sección. En ella, además de la posición del micrófono uno, se dispondrá de dos posiciones más formando un ángulo de $120^{\circ}$ entre sí, ver figura 3.2. Esta configuración sirve para realizar medidas simultáneas mediante tres micrófonos en la misma sección del conducto, con el fin de asegurar la independencia del punto de medida en sección y la validez del registro de presión en la cánula con la configuración de los micrófonos en línea.

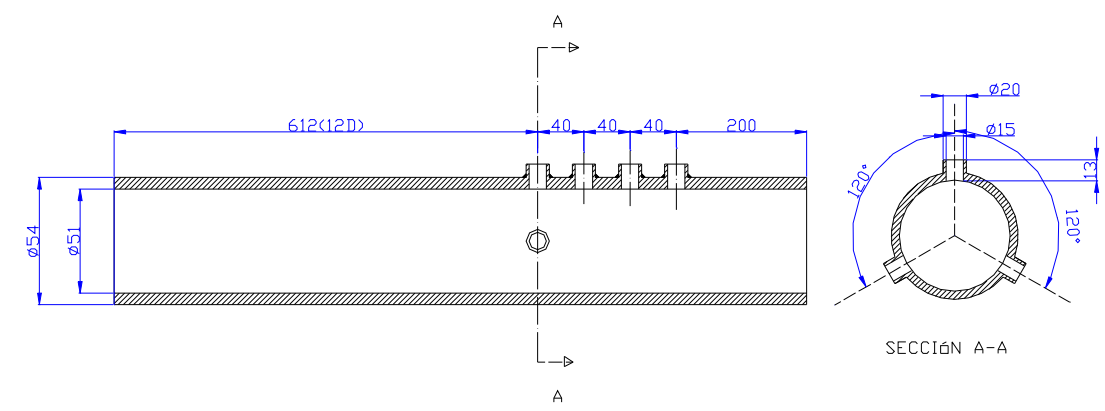

Figura 3.2: Disposición de los micrófonos en la cánula de salida del silenciador: en sección y en línea.

Estos micrófonos se encuentran perfectamente enrasados con la pared interior del conducto asegurando que no se cree ninguna cavidad o protuberancia que favoreciese la generación de vórtices en los puntos de medida, constituyéndose ellos mismos como fuentes adicionales de ruido. Aún así, esta posibilidad se considera por autores como Davies y Holland [48] como poco determinante ya que, debido al pequeño tamaño de los micrófonos empleados, $\frac{1}{4}$ " de diámetro $(\sim 7 \mathrm{~mm})$, las frecuencias características de estas cavidades saldrían fuera del rango de medida de interés. No obstante, la existencia de estas singularidades en el conducto pueden ser fuentes de inestabilidad, situación en la que no es tan evidente el hecho de que su efecto se límite a altas frecuencias y por tanto nos conduce de nuevo a la idea original de que es importante que los micrófonos se encuentren perfectamente enrasados.

Finalmente, es importante mencionar que los micrófonos escogidos para tales medidas, son micrófonos del tipo electrostático de electret 4935, adecuados para la medida de presión en cánula, los cuales poseen un limite de error 
respecto a la medida del espectro de presión sonora de $\pm 1 \mathrm{~dB}$ en el rango de frecuencias de 100 a $3 \mathrm{kHz}$ y de $\pm 2 \mathrm{~dB}$ en el rango de $3 \mathrm{kHz}$ a $5 \mathrm{kHz}$, errores que se minimizan calibrándolos cuidadosamente [38] antes de cada medida.

La medida de la temperatura del flujo se realiza mediante un termopar emplazado a la salida de la cánula, una vez finalizada la adquisición de los datos, de forma que mientras se registran los datos de presión en cánula y ruido radiado el termopar se retira de esta posición para evitar que su presencia distorsione los registros realizados. Esta medida de temperatura permitirá obtener una estimación de la velocidad del sonido y densidad del fluido que transita por la cánula, que junto con la medida del gasto medio determinará la velocidad media de dicho flujo.

\subsubsection{Método de cálculo de la intensidad radiada}

Para calcular la potencia sonora emitida a través de un campo de presión modal en un conducto es necesario tener en cuenta el efecto del flujo medio existente en el conducto [120]. La definición matemática de la potencia transmitida [57], $W_{t}$, que atraviesa una superficie $A_{s}$ es

$$
W_{t}=\int_{A_{s}} \mathbf{I} \cdot d \mathbf{A}_{s}
$$

donde el vector I denota el flujo de energía acústica por unidad de área o lo que es lo mismo el vector de intensidad acústica, que corresponde al producto escalar de las fluctuaciones de presión y velocidad asociadas a las partículas. Sin embargo, puesto que en un conducto con flujo la energía sonora también es transportada por éste adoptaremos una definición más general de la intensidad acústica en la que se preserven las propiedades de continuidad [124]

$$
\mathbf{I}=\left\langle h_{o}^{\prime} \cdot \mathbf{m}^{\prime}\right\rangle
$$

donde $\langle$.$\rangle se refiere al promediado temporal, h_{o}^{\prime}$ es la perturbación acústica de la entalpía de parada $h_{o}$, y $\mathbf{m}^{\prime}$ es la perturbación acústica del flujo de masa por unidad de área (momento lineal) $\mathbf{m}$, magnitudes cuyas expresiones son:

$$
h_{o}=h+\frac{\mathrm{u}^{2}}{2} \quad \mathrm{y} \quad \mathbf{m}=\rho \cdot \mathbf{u}
$$

donde $h$ es la entalpía específica del fluido, $\rho$ su densidad y $\mathbf{u}$ es la velocidad acústica de las partículas del fluido, magnitud que para el caso de un flujo monodimensional que se propaga en la dirección del eje del conducto y en el sentido del flujo puede escribirse como la suma de una componente media $U$, 
velocidad media del flujo, y una componente de perturbación v, del siguiente modo

$$
u=U+\mathrm{v}
$$

De las expresiones de $h_{o}(3.3)$ y $u(3.4)$, se deriva la ecuación para el cálculo de $h_{o}^{\prime}$ :

$$
h_{o}+h_{o}^{\prime}=h+h^{\prime}+\frac{(U+\mathrm{v})^{2}}{2}=\left(h+\frac{U^{2}}{2}\right)+\left(h^{\prime}+\frac{\mathrm{v}^{2}+2 U \mathrm{v}}{2}\right)
$$

en la que sí se desprecian los términos de segundo orden se obtiene la siguiente expresión para $h_{o}^{\prime}$

$$
h_{o}^{\prime}=h^{\prime}+U \mathrm{v}
$$

Considerando un flujo adiabático y teniendo en cuenta los principios de la termodinámica se tiene que la expresión de la fluctuación de la entalpía especifica es: $h^{\prime}=p^{\prime} / \rho_{j}+T s^{\prime}$, donde $p^{\prime}$ y $s^{\prime}$ representan la fluctuación de la presión acústica y entropía específica respectivamente, siendo $T$ la temperatura absoluta del fluido y $\rho_{j}$ la densidad del fluido. Si sustituimos esta ecuación en la expresión de $h_{o}^{\prime}(3.6)$ y suponemos un flujo isoentrópico $\left(s^{\prime}=0\right)$ obtenemos

$$
h_{o}^{\prime}=p^{\prime} / \rho_{j}+U \mathrm{~V}
$$

Mediante un procedimiento similar se obtiene el valor $\mathbf{m}^{\prime}$ empleando las expresiones de m (3.3) y v (3.4), considerando que la dirección y sentido de los vectores es la del flujo medio en el conducto, como se a mencionado anteriormente

$$
m+m^{\prime}=\left(\rho_{j}+\rho^{\prime}\right) \cdot(U+\mathrm{v})=\rho_{j} U+\left(\rho_{j} \mathrm{v}+\rho^{\prime} U+\rho^{\prime} \mathrm{v}\right)
$$

despreciando al igual que antes los términos de segundo orden la ecuación queda finalmente como

$$
m^{\prime}=\rho_{j} \mathrm{v}+\rho^{\prime} U
$$

donde $\rho^{\prime}$ representa la fluctuación acústica de la densidad y está relacionada con la fluctuación de la presión acústica, mediante la expresión $p^{\prime}=\rho^{\prime} c^{2}$, siendo $c$ la velocidad del sonido a la temperatura del flujo. Realizando todas estas sustituciones en la ecuación (3.2) la intensidad acústica en un conducto con flujo medio, en la dirección y sentido del flujo, puede escribirse como sigue [120]

$$
I=\left\langle p^{\prime} \cdot \mathrm{v}\right\rangle+\frac{U}{\rho_{j} c^{2}}\left\langle p^{\prime} \cdot p^{\prime}\right\rangle+\frac{U^{2}}{c^{2}}\left\langle p^{\prime} \cdot \mathrm{v}\right\rangle+\rho_{j} U\langle\mathrm{v} \cdot \mathrm{v}\rangle
$$


Considerando la definición del valor medio de un producto escalar, $\langle A \cdot B\rangle=$ $=\operatorname{Re}\left[A \cdot B^{*}\right]=\left[A \cdot B^{*}+A^{*} \cdot B\right] / 2$, donde ()$^{*}$ representa el complejo conjugado de dicha magnitud, introduciendo la definición del número de Mach medio del flujo, $M=U / c$, y representando la fluctuación de presión $p^{\prime}$ por $p$, a fin de unificar posteriores notaciones, se llega a que la intensidad acústica transmitida a través de la sección $A_{s}$ responde a la ecuación

$$
I=\operatorname{Re}\left[p u^{*}\left(1+M^{2}\right)\right]+\frac{M}{\rho_{j} c}\left[|p|^{2}+\left(\rho_{j} c\right)^{2}|\mathrm{v}|^{2}\right]
$$

Si suponemos que existe propagación de un único modo acústico, que dentro del rango de frecuencias de interés corresponde a considerar que el campo acústico está generado por una onda plana [127] en el conducto, a la que se le superpone un flujo medio uniforme, las componentes espectrales de las fluctuaciones acústicas de presión, $\widehat{p}(x, \omega)$, y velocidad, $\mathrm{v}(x, \omega)$, en cada punto del conducto pueden expresarse como suma de las componentes de presión incidente (en la dirección del flujo) y reflejada (en dirección opuesta al flujo), de amplitudes espectrales complejas $\widehat{p}^{+}$y $\widehat{p}^{-}$respectivamente, mediante las ecuaciones [40]

$$
\begin{aligned}
\widehat{p}(x, \omega) & =\widehat{p}^{+} \cdot \exp \left(-j \beta^{+} x\right)+\widehat{p}^{-} \cdot \exp \left(j \beta^{-} x\right) \\
\rho_{j} c \mathrm{v}(x, \omega) & =\widehat{p}^{+} \cdot \exp \left(-j \beta^{+} x\right)-\widehat{p}^{-} \cdot \exp \left(j \beta^{-} x\right)
\end{aligned}
$$

siendo:

$$
\beta^{+}=\frac{k+\alpha(1-j)}{1+M} \quad \text { y } \quad \beta^{-}=\frac{k+\alpha(1-j)}{1-M}
$$

los números de onda complejos incidente y reflejado respectivamente, corregidos con el efecto del flujo medio a través del número de Mach $M, k=\omega / c$ número de onda acústico, y $\alpha$ el coeficiente de atenuación viscotérmico definido como:

$$
\alpha=\frac{1}{a \cdot c} \cdot\left(\frac{\nu \omega}{2}\right)^{1 / 2}\left[1-(\gamma-1) P_{r}^{-0.5}\right]
$$

donde $a$ es el radio del conducto, $\nu$ la viscosidad cinemática, y $P_{r}$ el número de Prandtl. Sin perdida de generalidad puede escogerse $x=0$, obteniéndose:

$$
\widehat{p}(x, \omega)=\widehat{p}^{+}+\widehat{p}^{-} \quad \text { y } \quad u(x, \omega)=\frac{\widehat{p}^{+}-\widehat{p}^{-}}{\rho_{j} c}
$$

De esta forma la intensidad neta transmitida obtenida en la ecuación (3.11) puede calcularse a partir de las componentes de las ondas incidente y reflejada, teniendo en cuenta la siguiente igualdad

$$
\operatorname{Re}\left[p \mathrm{v}^{*}\right]=\frac{\left[p \mathrm{v}^{*}+p^{*} \mathrm{v}\right]}{2}=\frac{1}{2 \rho_{j} c}\left[\left|\widehat{p}^{+}\right|^{2}-\left|\widehat{p}^{-}\right|^{2}\right]
$$


Sustituyendo la ecuación (3.17) en la ecuación (3.11), la intensidad acústica neta transmitida en el conducto en presencia de flujo medio, puede escribirse finalmente como [48]

$$
I=\frac{1}{2 \rho_{j} c}\left[\left|\widehat{p}^{+}\right|^{2}(1+M)^{2}-\left|\widehat{p}^{-}\right|^{2}(1-M)^{2}\right]
$$

De la expresión anterior, se deduce que el factor más determinante en la obtención de la intensidad transmitida es la determinación de las componentes de las ondas incidente y reflejada, por lo que a continuación se presentan diferentes algoritmos de descomposición a fin de valorar las ventajas e inconvenientes de cada uno de ellos.

\subsection{Teoría de la descomposición de ondas}

Como se concluye de la sección anterior es necesario disponer de una buena estimación de las componentes, incidente y reflejada, de la onda de presión en el interior del conducto, ya que constituyen la hipótesis de base para el cálculo de la intensidad neta transmitida en el interior del conducto. Con el objetivo de garantizar que esta hipótesis posee una fiabilidad adecuada nos centraremos, en primer lugar, en el método empleado para la determinación de ambas componentes. Su validez y potencia se justificará mediante su correlación directa frente a otros modelos existentes en la literatura y ampliamente verificados.

Las componentes incidente y reflejada de las señales de presión registradas en el interior de un conducto se han evaluado tradicionalmente mediante el método de los dos micrófonos, de uso ampliamente extendido y que se presenta en el apartado siguiente. Sin embargo, dicho método posee varios problemas; entre ellos, quizá uno de los más destacados sea el efecto eventual que puede tener el ruido sobre las medidas de presión, el cual puede ser más crítico en un caso no lineal [52], ya que en este método de medida se desprecia la existencia de señales interferentes [26], cuya consideración supondría disponer al menos de un micrófono más.

Por ello, la principal cualidad que posee el método de descomposición propuesto en nuestro método de estimación es la de ser una técnica aplicable a múltiples sensores, no únicamente a dos, empleando los nuevos grados de libertad introducidos en el sistema para optimizar o al menos minimizar efectos no deseados, como ruido, o los derivados de trabajar a temperaturas extremas. El método escogido emplea un algoritmo de descomposición basado en la técnica del "beamforming", técnica que se basa en el tratamiento 
de matrices a semejanza de lo que se hace en el estudio de antenas de ondas electromagnéticas. Este método posee un inconveniente importante, y es el de ser altamente matemático en comparación con otras técnicas de descomposición, pero presenta la ventaja de poseer una excelente robustez, lo que justifica su empleo en aplicaciones complejas aunque los principios físicos queden algo escondidos.

\subsubsection{Método de los dos micrófonos}

Se parte de la suposición de que por debajo de la frecuencia de corte a la que empiezan a propagarse los modos de orden superior, la propagación de la energía acústica a lo largo de un conducto uniforme puede restringirse sin pérdida de generalidad a un movimiento unidimensional (ondas planas). Esta frecuencia de corte correspondiente a la frecuencia a partir de la cual se propaga el primer modo transversal, responde a valores del número de Helmholtz de $k a=1.84 \sqrt{1-M^{2}}$ [51]. Este límite corresponde a unos $4000 \mathrm{~Hz}$ para un conducto de $51 \mathrm{~mm}$ de diámetro a temperaturas y velocidades típicas de funcionamiento, alcanzando los $6500 \mathrm{~Hz}$ para conductos de menor diámetro (30 mm); de este modo, bajo la condición de que el rango de frecuencias de interés quede dentro de tales límites, la restricción de onda plana es válida, lo que ofrece una simplificación considerable tanto del procedimiento de medida como de su posterior análisis.

Autores como Holland y Davies han desarrollado una metodología de descomposición basada en la medida de la presión sonora mediante dos micrófonos emplazados en la pared de la cánula [48], separados una distancia $d$, como se muestra en la figura 3.3. Al asumir movimiento unidimensional de las ondas las fluctuaciones de presión y velocidad acústica en cada punto pueden expresarse como la suma de las componentes incidente y reflejada a través de las ecuaciones (3.12) y (3.13), como ya se comentó en la sección anterior.

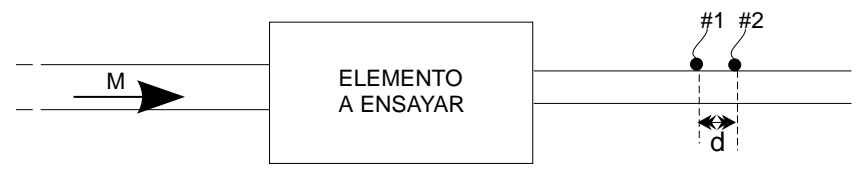

Figura 3.3: Geometría del sistema de medida con la técnica de los dos micrófonos.

Asignando arbitrariamente $x=0$ (el origen de fases) a la posición del micrófono 1 , si el micrófono 2 está a una distancia $d$ del primer micrófono, la presión en los dos micrófonos puede escribirse como:

$$
\widehat{p}_{1}(\omega)=\widehat{p}^{+}+\widehat{p}^{-} \quad \text { y } \quad \widehat{p}_{2}(\omega)=\widehat{p}^{+} \cdot \exp \left(-j \beta^{+} d\right)+\widehat{p}^{-} \cdot \exp \left(j \beta^{-} d\right)
$$


De las ecuaciones anteriores se puede obtener la expresión del espectro cruzado entre ambos micrófonos, $G_{12}$ y la del autoespectro de la presión medida por el micrófono $1, G_{11}$, quedando

$$
G_{12}=\widehat{p}_{1}^{H} \cdot \widehat{p}_{2} \quad \text { y } \quad G_{11}=\widehat{p}_{1}^{H} \cdot \widehat{p}_{1}
$$

donde $\langle.\rangle^{H}$ representa al transpuesto conjugado del vector.

Dividiendo ambas ecuaciones anteriores y definiendo el coeficiente de reflexión como $R(\omega)=\widehat{p}^{-} / \widehat{p}^{+}$se tiene:

$$
\frac{G_{12}}{G_{11}}=\frac{\exp \left(-j \beta^{+} d\right)+R(\omega) \cdot \exp \left(j \beta^{-} d\right)}{1+R(\omega)}
$$

expresión a partir de la que se deriva una expresión analítica del coeficiente de reflexión en función de dos cantidades medidas, $G_{11}$ y $G_{12}$, conocidos los valores de $k, M$ y $a$ :

$$
R(\omega)=-\left\{\frac{G_{12}-G_{11} \exp \left(-j \beta^{+} d\right)}{G_{12}-G_{11} \exp \left(j \beta^{-} d\right)}\right\}
$$

Considerando la definición del autoespectro del primer micrófono, $G_{11}$ (3.20) y la del coeficiente de reflexión se obtienen las componentes de la onda incidente y reflejada:

$$
\left|\widehat{p}^{+}\right|=\frac{\sqrt{G_{11}}}{|1+R(\omega)|} \quad \text { y } \quad \widehat{p}^{-}=R(\omega) \cdot \widehat{p}^{+}
$$

El hecho de que la ecuación (3.23) contenga únicamente el modulo de $\widehat{p}^{+}$no supone ningún problema ya que la fase de $\widehat{p}^{+}$se escogía arbitrariamente como cero sin perdida de generalidad, mientras que $\widehat{p}$ es complejo en general. Debe resaltarse sin embargo que la eficacia de la descomposición depende de cómo sean de coherentes las cantidades $\widehat{p}^{+}$y $\widehat{p}^{-}$[48], ya que la presencia del flujo medio inducido en el sistema u otras fuentes de ruido incoherente podrían comprometer los resultados de las estimaciones espectrales realizadas a través de ellas.

Este método ofrece la ventaja de poder obtener la señal incidente y reflejada de forma analítica, lo que no ocurre en el caso de emplear más de dos micrófonos, como se verá en la sección posterior, debido al mayor número de grados de libertad introducido en el sistema a resolver, transformándolo en un sistema sobredimensionado. Además tiene la cualidad de introducir las correcciones con los efectos de atenuación viscosa de la onda sonora, los cuales, en el caso de no ser considerados, constituyen una fuente de error adicional para la propagación de esta onda en presencia de flujo, como ponen de manifiesto Åbom y Bodén [1]. 


\subsubsection{Descomposición de ondas mediante técnicas de confor- mación del haz.}

Ya han sido mencionados los problemas que presenta el método de descomposición de la onda de presión en sus componentes incidente y reflejada mediante el uso de dos micrófonos, de entre los cuales destaca la no inclusión de posibles señales interferentes en el sistema [26], lo que puede desvirtuar los resultados y para lo cual sería necesario disponer de al menos un micrófono más, que al introducir nuevos grados de libertad sobredimensiona el sistema. Como respuesta a esta necesidad se opta por una técnica basada en el tratamiento estadístico de los datos, la cual se desarrolla y explica a continuación.

En particular, en el caso que presentamos se ha empleado el registro de cuatro micrófonos emplazados en la cánula de escape, tal y como se describe en la sección 3.2.1. El fin es, por un lado, mejorar la descomposición de la señal de presión mejorando la relación señal-ruido, cuya mala calidad es una de las consecuencias de la presencia de flujo en las medidas, disminuyendo ésta a medida que aumenta el flujo. Y por otro lado tener en cuenta la influencia de la turbulencia, factor muy importante en conductos con flujos subsónicos, como es nuestro caso, pudiendo causar una desviación en la función de transferencia medida para dos micrófonos.

Como ya se ha mencionado en la introducción de esta sección, la base del desarrollo del método de descomposición es la técnica de procesado en array, denominada beamforming o conformación de haz. El beamforming es una técnica de procesado espacial empleada para la estimación de la forma de onda de la señal incidente sobre un conjunto (array) de sensores desde una dirección dada. Los beamformers (conformadores del haz) pueden considerarse como filtros espaciales que combinan las salidas de un array de sensores para formar un haz que señala en la dirección de la fuente deseada.

Para la aplicación de la técnica del beamforming a la descomposición de las señales medidas en una cánula se deben tener en cuenta las siguientes consideraciones [138]:

- Propagación lineal de la presión y velocidad del sonido, considerando que la apertura del array (longitud total) es pequeña comparada con la longitud de onda.

- Diferentes ángulos de incidencia del flujo incidente y reflejado sobre el array de sensores.

Comenzaremos anotando una formulación general sobre el algoritmo de descomposición empleado y posteriormente se razonará por qué se ha escogido 
este algoritmo particular para implementar el beamforming, indicando cuales son sus características más importantes.

El filtrado espacial de las señales procedentes del array de sensores, que se realiza mediante la técnica de procesado en array denominada beamforming, se basa en la ponderación de la señal medida con cada uno de los captadores de la serie, por medio de unos factores de peso $w_{n}$, mediante los cuales se modula la información recibida, lo que constituye el denominado beamformer de banda estrecha mostrado en la figura 3.4.

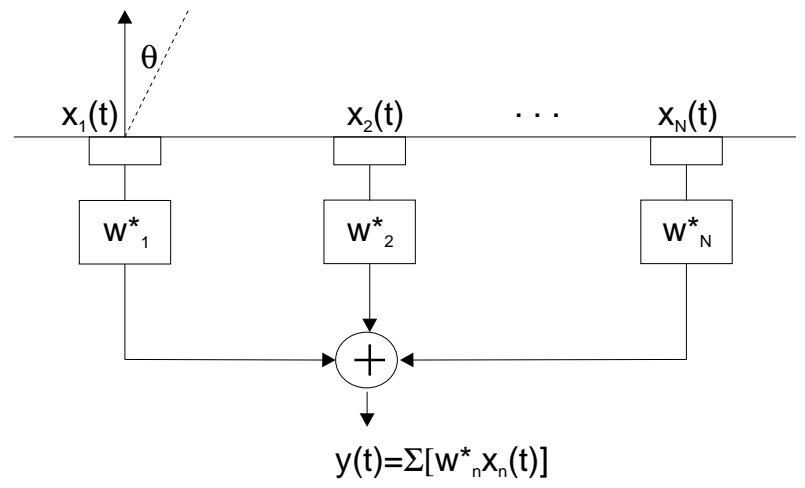

Figura 3.4: Conformador del haz (beamformer) de banda estrecha. La dirección de llegada $\theta$ es el ángulo entre la dirección de la fuente y un eje perpendicular al conducto.

a)

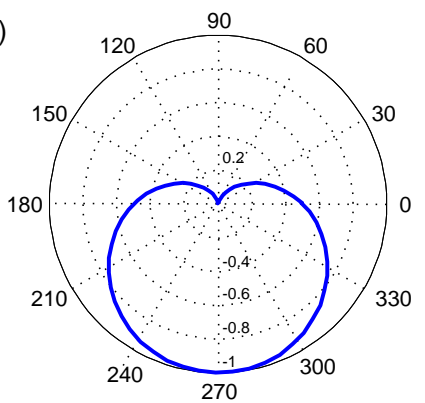

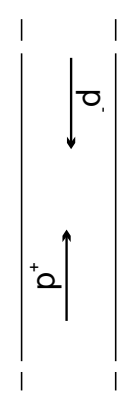

Figura 3.5: Diagrama de recepción para obtener la señal: (a) incidente y (b) reflejada.

Para aplicar la técnica del beamforming a las ondas de presión propagadas a lo largo de la cánula de escape, se considera que éstas ondas son señales de banda ancha con picos armónicos indeterminados. Por ello, en primer lugar consideraremos el caso de la aplicación de la técnica a una perturbación de presión monocromática, pasando posteriormente a generalizar su expresión para una señal de banda ancha. 
De acuerdo con la figura 3.4, la señal de salida del beamformer en el instante $t$ viene dada por la expresión:

$$
y(t)=\sum_{n=1}^{N} w_{n}^{*} x_{n}(t)
$$

donde $N$ es el número de sensores, $w_{n}$ y $x_{n}(t)$ son el peso y la señal de salida instantánea del transductor n-ésimo respectivamente, y $(.)^{*}$ se refiere al conjugado.

Suponiendo que se recibe una onda plana de frecuencia $f$, con una velocidad $c$, y una dirección de llegada $\theta, s(t)=s_{0}(t) \cdot \exp (j 2 \pi f t)$, que llega en el instante $t$ al array, y tomando la posición del primer transductor como el origen de fases, la salida del transductor n-ésimo será (ver figura 3.7):

$$
x_{n}(t)=s_{0}(t) \exp \left(j 2 \pi f\left[t-\tau_{n}\right]\right) \quad \forall n \in[1, N]
$$

donde $\tau_{n}=\left(d_{n} \sin \theta / c\right)$ es el tiempo que tarda la señal en llegar al sensor nésimo, siendo $d_{n}$ la distancia del sensor n-ésimo al primer sensor. En particular, si todos los sensores están igualmente espaciados con separación $d$ (ver figura $3.7)$, y se escoge el origen de distancias en el primer sensor $\left(d_{1}=0\right), \tau_{n}$ será $\tau_{n}=((n-1) \cdot d \sin \theta / c) \equiv(n-1) \cdot \tau_{d, \theta}$. Introduciendo finalmente la frecuencia angular $\omega=2 \pi f$, la ecuación (3.25) se reescribe como:

$$
x_{n}(t)=s_{0}(t) \exp \left(j \omega\left[t-(n-1) \tau_{d, \theta}\right]\right)=s(t) \cdot \exp \left[-j \omega(n-1) \tau_{d, \theta}\right]
$$

Sustituyendo la expresión (3.26) en la ecuación (3.24) de la señal de salida del beamformer en el instante $t$, obtenemos:

$$
y(t)=s(t) \sum_{n=1}^{N} w_{n}^{*} \cdot \exp \left[-j \omega(n-1) \tau_{d, \theta}\right] \equiv s(t) \cdot H(\theta, f)
$$

siendo $H(\theta, f)$ la respuesta del beamformer, es decir, su función de transferencia frente a ondas planas, lo que permite definir el beampattern como el módulo al cuadrado de $H(\theta, f)$, cuya representación gráfica proporciona la forma del diagrama de recepción que sintetiza el vector de pesos. Con el fin de interpretar más claramente $H(\theta, f)$, en la figura 3.5 están recogidos los diagramas de recepción para la señal incidente y la señal reflejada, respectivamente. En esta figura se puede observar como para descomponer las señales de presión medidas en la cánula, en sus componentes incidente y reflejada, se conforma el diagrama de recepción del array de forma que obtengamos el máximo de este diagrama en la dirección de llegada de la onda escogida (incidente o reflejada), 
a la vez que se anulen las señales procedentes de otras direcciones no deseadas. Para ello se escogen los pesos $w_{n}$, de forma que la energía procedente de una cierta dirección del espacio sea preferentemente recibida por el array, mientras que cualquier otra fuente que esté afectando al array desde otra dirección será atenuada, es decir, realizar un filtrado espacial de forma que sólo dejase pasar la señal incidente (figura 3.5(a)) ó la señal reflejada (figura 3.5(b)).

Esta función respuesta, $H(\theta, f)$, puede expresarse de forma vectorial como:

$$
H(\theta, f)=\mathbf{w}^{H} \cdot \mathbf{a}(\theta)
$$

Donde $(.)^{H}$ hace referencia al transpuesto conjugado del vector de pesos $\mathbf{w}=$ $\left[w_{1}, \ldots, w_{N}\right]^{T}$, y $\mathbf{a}(\theta)$ es el vector de respuesta de array o vector de dirección de la señal de llegada, cuya expresión es:

$$
\mathbf{a}(\theta)=\left[1, \exp \left(-j \omega \tau_{d, \theta}\right), \ldots, \exp \left(-j \omega(N-1) \tau_{d, \theta}\right)\right]^{T}
$$

Por definición $H(\theta, f)$ es el resultado del producto escalar del vector de pesos conjugado por el vector de dirección asociado a la señal de llegada (3.28), por lo que la respuesta $H(\theta, f)$ va a depender del ángulo formado en el espacio $\mathrm{N}$-dimensional por ambos vectores. De modo que sí para una señal que llegue en una dirección $\theta$ y con una frecuencia $f$, el ángulo entre dichos vectores fuese $90^{\circ}$ (ortogonales), entonces la respuesta $H(\theta, f)$ sería nula y si por el contrario, el ángulo fuese $0^{\circ}$, el módulo de la respuesta sería máximo. Por tanto, la capacidad que tendrá el beamformer de discriminar dos fuentes definidas por pares $\left(\theta_{1}, f_{1}\right)$ y $\left(\theta_{2}, f_{2}\right)$ está fijada por la distancia entre ambas fuentes, es decir, por el ángulo que formen sus vectores directores. De modo que cuanto más próximos estén, más semejante será su respuesta dada por su ángulo respecto a $\mathbf{w}$.

Esto conlleva a que si el objetivo es realizar un filtrado espacial en el que dejemos pasar la fuente $\left(\theta_{1}, f_{1}\right)$ y anulemos la fuente $\left(\theta_{2}, f_{2}\right)$, mejor será este filtrado cuanto más separados estén $\theta_{1}$ y $\theta_{2}$ para que el ángulo entre $\mathbf{a}\left(\theta_{1}\right)$ y $\mathbf{a}\left(\theta_{2}\right)$ sea lo suficientemente grande como para que sus respuestas sean lo más independientes posibles. En el caso particular considerado, ondas en un conducto, las señales que queremos filtrar provienen de $\theta_{1}=-90^{\circ}$ y $\theta_{2}=90^{\circ}$, por tanto las señales incidente y reflejada, serán perfectamente discriminadas por el beamformer, ya que los vectores de dirección asociados forman un ángulo de $180^{\circ}$.

Por otro lado, la generalización a las señales de banda ancha resulta ahora inmediata, como se muestra en la figura 3.6, a través de los siguientes pasos: 


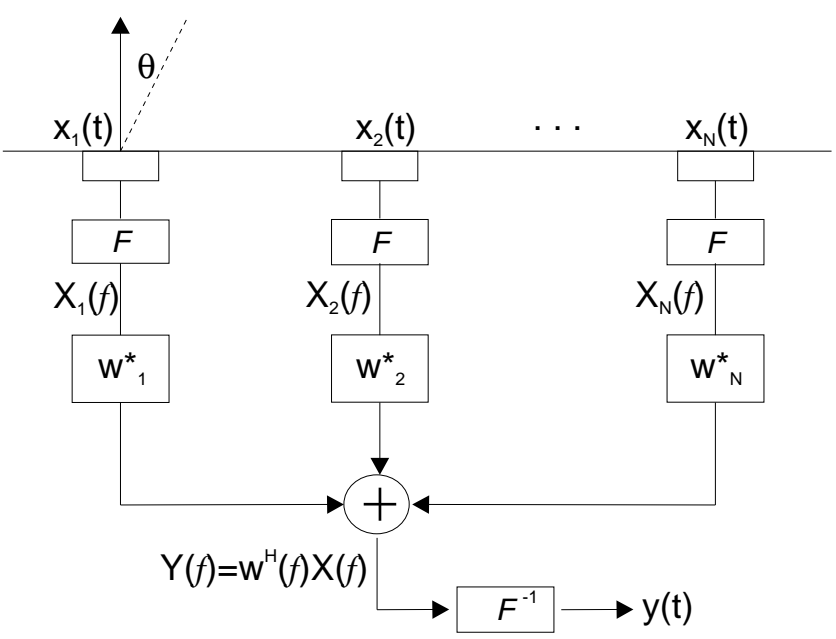

Figura 3.6: Implementación en el dominio frecuencia de un conformador del haz (beamformer) de banda ancha.

- En primer lugar se obtienen las Transformadas de Fourier de las señales de banda ancha recibidas en cada sensor.

- En segundo lugar de cada raya espectral (de la transformada de Fourier de la salida de cada sensor) correspondiente a la misma frecuencia, se realiza el filtrado espacial como si fuera de banda estrecha. De este filtrado se habrán obtenido las salidas deseadas en la descomposición, en nuestro caso la señal incidente y reflejada en dominio frecuencia.

- Finalmente la obtención de las señales incidente y reflejada en dominio tiempo se realiza a través de la transformada de Fourier inversa, de las señales filtradas.

Todo este proceso está perfectamente detallado en el artículo de Piñero et al [138], por lo que no se profundizará más en él, con el fin de no salirnos del objetivo de la sección que es el de mostrar las cualidades de este método de descomposición frente al método de descomposición con dos micrófonos.

Una última cuestión a tener en cuenta son los efectos del muestreo espacial, en particular el problema de aliasing, éste se refiere al solapamiento que puede ocurrir en el muestreo espacial similar al que ocurre en el muestreo temporal, y que se debe a la distancia escogida entre los captadores de presión. Este fenómeno ocurre cuando la frecuencia de muestreo espacial $\left(f_{s}\right)$, cuyo periodo depende de la separación entre los sensores, no cumple el criterio 
de Nyquist siguiente:

$$
f_{s} \geq 2 \cdot f_{\text {onda }}
$$

donde $f_{\text {onda }}$ es la frecuencia espacial de la onda. Esto significaría que si los sensores se encuentran todos equidistantes una distancia $d$ y que la onda plana llega en una dirección $\theta$ con velocidad de propagación $c$, se debe cumplir (basándonos en la figura 3.7) que [156]

$$
f_{\text {onda }} \leq \frac{f_{s}}{2}=\frac{1}{2 T_{d}}=\frac{c}{2 d \sin \theta}
$$

donde $T_{d}=\frac{d \sin \theta}{c}$ es el periodo de muestreo espacial de la señal.

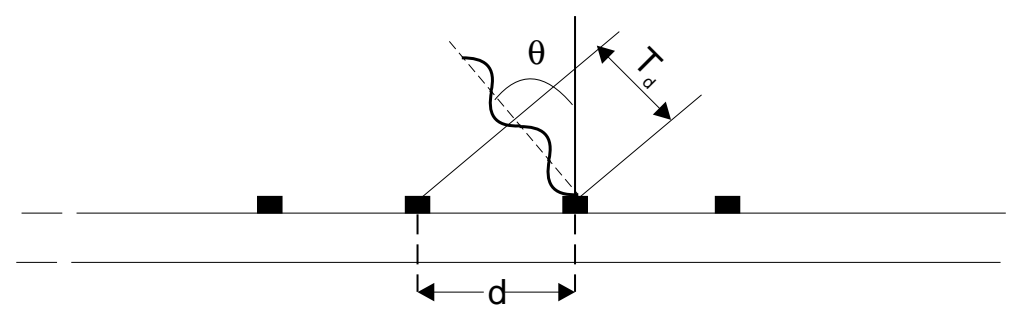

Figura 3.7: Cálculo del periodo de muestreo espacial.

Por otro lado puede ocurrir que los vectores asociados a dos o más señales de banda estrecha sean iguales, $\mathbf{a}\left(\theta_{1}\right)=\mathbf{a}\left(\theta_{2}\right)$, por lo que todas tendrán la misma respuesta del beamformer. A este efecto se le llama ambigüedad y puede suceder cuando el espaciado entre los sensores es muy grande. Existe otro tipo de ambigüedad cuando el espaciado entre los sensores es muy pequeño, debido a que en este caso los vectores de dirección de las ondas recibidas no están lo suficientemente dispersos en el espacio N-dimensional, lo que degrada la discriminación espacial del beamformer.

En todo el desarrollo presentado queda patente que los beamformers (tratamientos de descomposición) actúan como filtros espaciales que modifican la información captada por los sensores mediante la correcta selección de los factores de ponderación, fijando una dirección de captación en la que los sensores deben detectar preferentemente la señal de perturbación.

Finalmente, restaría mencionar como se obtienen estos factores de peso $w_{n}$. Para ello, de los dos tipos de beamformers existentes (independientes de los datos y óptimos estadísticamente), se ha escogido un beamformer óptimo estadísticamente denominado L.C.M.V. (Linearly Constrained Minimun Variance) o conformador de haz de mínima varianza con restricciones lineales. Ya que la finalidad de este tipo de beamformers es optimizar su respuesta de 
forma que se minimicen las contribuciones provenientes de otras direcciones no deseadas y las posibles contribuciones de ruido al sistema, explotando las propiedades estadísticas de las señales y el ruido [138].

\subsubsection{Conformador del haz de mínima varianza con restriccio- nes lineales (LCMV)}

La idea básica del L.C.M.V. es forzar la respuesta del beamformer para que las señales de la dirección de interés pasen con una ganancia y fase especificadas. En el caso de ondas planas en tubos no hay más que una dirección de propagación, por tanto, será necesario elegir los coeficientes de manera que la recepción sea máxima en un sentido (incidente ó reflejado) y nula en el opuesto.

Teniendo en cuenta que la respuesta del beamformer a una onda procedente de la dirección $\theta$ y frecuencia $f$ viene dada por la expresión $\mathbf{w}^{H} \mathbf{a}(\theta)$ (ecuación (3.28)), el algoritmo $L C M V$ se diseña para que los pesos satisfagan la siguiente restricción

$$
\mathbf{w}^{H} \cdot \mathbf{a}\left(\theta_{o}, f_{o}\right)=g
$$

donde $g$ es una constante compleja, que asegura que cualquier señal procedente de la dirección $\theta_{o}$ con frecuencia $f_{o}$ pasa a la salida del beamformer con ganancia $g$. Por otro lado, se deben minimizar las contribuciones a la salida del beamformer de las señales interferentes (señales no procedentes de la dirección $\theta_{o}$ con frecuencia $f_{o}$ ). Para ello, se eligen los pesos de modo que minimicen el valor esperado del módulo de la señal de salida, $E\left(\left|y^{2}\right|\right)$, o lo que es lo mismo, su potencia media y estén sujetos a la restricción anterior:

$$
E\left(\left|y^{2}\right|\right)=\min _{w}\left\{\mathbf{w}^{H} \cdot \Sigma_{x} \cdot \mathbf{w}\right\} \text { sujetos a } \mathbf{a}^{H}(\theta) \mathbf{w}=g^{*}
$$

donde $\Sigma_{x}$ es la matriz de covarianza de los datos, matriz que representa la coherencia que existe entre las medidas tomadas por cada sensor con respecto al resto. Matriz que para cada frecuencia $f_{k}$ se define

$$
\Sigma_{x}\left(f_{k}\right)=E\left[\mathbf{X}\left(f_{k}\right) \cdot \mathbf{X}^{H}\left(f_{k}\right)\right]
$$

Siendo $\mathbf{X}\left(f_{k}\right)$ la componente k-ésima de la transformada de Fourier discreta de $\mathbf{x}(t)=\left[x_{1}(t), \ldots, x_{N}(t)\right]^{T}$ vector salida del array de sensores.

Para resolver el problema planteado (ecuación (3.33)) y obtener el vector de pesos para el algoritmo $L C M V$ se emplea el método de multiplicadores de Lagrange [138], obteniéndose la siguiente expresión:

$$
\mathbf{w}=g^{*} \frac{\Sigma_{x}^{-1} \cdot \mathbf{a}(\theta)}{\mathbf{a}(\theta)^{H} \cdot \Sigma_{x}^{-1} \cdot \mathbf{a}(\theta)}
$$


En la ecuación (3.35) se puede observar que es necesario que la matriz de covarianza sea invertible para obtener los vectores de pesos adecuados. Para señales sin ruido incorrelado, la matriz puede tener rango inferior al número de sensores, $N$, por lo que no será invertible, ya que para que una matriz $N \times N$ pueda ser invertible, entre otras cosas, debe poseer rango $N$, lo cual se logra siempre con señales inmersas en ruido no correlado con ellas. Si no fuera así, bastará con sumarle a la diagonal principal de la matriz de covarianza un porcentaje pequeño de su traza.

Por tanto el filtrado espacial $L C M V$ optimiza la salida del beamformer con respecto a la relación señal-ruido e interferencias [138], ya que en general las señales procedentes del array no están correladas unas con otras y a su vez con el ruido. En este sentido, ésta es la técnica óptima para estimar la forma de la onda deseada en presencia de ruido blanco Gausiano e interferencias incorreladas. Además, cuando se escoge $g$ igual a 1 el vector de pesos obtenido se denomina respuesta de mínima varianza de distorsión del beamformer, ya que en este caso la señal de interés pasa sin atenuación ni amplificación.

Se puede generalizar este problema al caso en que se posean varias restricciones lineales para poder controlar la respuesta, como es el caso que nos atañe, donde la señal de presión que se registra en la cánula de escape se quiere descomponer en sus componentes incidente y reflejada, las cuales provienen de $\theta=-90^{\circ}$ y $\theta=90^{\circ}$, respectivamente. Por tanto, para cada señal conocida la distancia entre los sensores, $d$, la dirección de incidencia, $\theta$, la velocidad de propagación, $c$, y la frecuencia correspondiente a cada raya espectral, podemos generar sus vectores de dirección $\mathbf{a}(\theta)$ (ecuación (3.29)); éstos serán diferentes para las componentes incidente, $\mathbf{a}^{+}(\theta)$, y reflejada, $\mathbf{a}^{-}(\theta)$, poseyendo así el problema dos restricciones. Además como existe flujo medio en el conducto los números de onda correspondientes a la propagación incidente y reflejada, vendrán dados por la ecuación (3.14), y por tanto serán diferentes para ambas componentes de dirección, de modo que estos vectores de dirección vendrán dados por las siguientes expresiones:

$$
\begin{aligned}
& \mathbf{a}^{+}(\theta)=\mathbf{a}\left(-90^{\circ}\right)=\left[1, \exp \left(j \beta^{+} d\right), \ldots, \exp \left(j(N-1) \beta^{+} d\right)\right]^{T} \\
& \mathbf{a}^{-}(\theta)=\mathbf{a}\left(90^{\circ}\right)=\left[1, \exp \left(-j \beta^{-} d\right), \ldots, \exp \left(-j(N-1) \beta^{-} d\right)\right]^{T}
\end{aligned}
$$

Con estas restricciones, se generaliza el problema del cálculo de pesos definiendo una matriz de restricciones $\mathbf{A}(\boldsymbol{\Theta})$, cuyo valor depende de la componente a filtrar. Así, por ejemplo, para filtrar la señal incidente eliminando la reflejada, la matriz de restricciones que se adoptaría sería:

$$
\mathbf{A}(\boldsymbol{\Theta})=\left[\begin{array}{ll}
\mathbf{a}^{+}(\theta) & \mathbf{a}^{-}(\theta)
\end{array}\right]^{T}
$$


cuyo vector respuesta deseado, g, para obtener la señal incidente sería

$$
\mathbf{g}^{+}=\left[\begin{array}{ll}
1 & 0
\end{array}\right]^{T}
$$

y el vector de pesos para obtener la señal incidente, $\mathbf{w}^{+}$, correspondería a la siguiente ecuación:

$$
\mathbf{w}^{+}=\mathbf{g}^{+}\left[\Sigma_{x}^{-1} \cdot \mathbf{A}^{H}(\boldsymbol{\Theta}) \cdot\left[\mathbf{A}^{H}(\boldsymbol{\Theta})^{H} \cdot \Sigma_{x}^{-1} \cdot \mathbf{A}^{H}(\boldsymbol{\Theta})\right]^{-1}\right]
$$

De forma análoga se obtendría el vector de pesos para la señal reflejada $\mathbf{w}^{-}$, teniendo en cuenta que ahora su vector respuesta $\mathbf{g}^{-}$es: $\mathbf{g}^{-}=\left[\begin{array}{ll}0 & 1\end{array}\right]^{T}$, quedando la expresión del vector de pesos para la señal reflejada como

$$
\mathbf{w}^{-}=\mathbf{g}^{-}\left[\Sigma_{x}^{-1} \cdot \mathbf{A}^{H}(\boldsymbol{\Theta}) \cdot\left[\mathbf{A}^{H}(\boldsymbol{\Theta})^{H} \cdot \Sigma_{x}^{-1} \cdot \mathbf{A}^{H}(\boldsymbol{\Theta})\right]^{-1}\right]
$$

Finalmente, haciendo uso de los vectores de peso obtenidos para las señales incidente (3.40) y reflejada (3.41) podemos filtrar convenientemente la señal de origen y obtener las señales incidentes y reflejadas, a través de la definición de la respuesta del beamformer (3.24) en dominio frecuencia.

$$
\mathbf{Y}^{+}\left(f_{k}\right)=\mathbf{w}^{+H} \mathbf{X}\left(f_{k}\right) \quad \text { y } \quad \mathbf{Y}^{-}\left(f_{k}\right)=\mathbf{w}^{-H} \mathbf{X}\left(f_{k}\right) \quad k \in\left[1, N_{m}\right]
$$

$\mathrm{Al}$ realizar todo este proceso para todas las rayas espectrales obtendremos como resultado el espectro de las señales incidente y reflejada, obteniendo su expresión en el dominio del tiempo calculando las transformadas de Fourier inversas de sus correspondientes espectros.

\subsubsection{Consistencia del método de descomposición}

Una vez descrito el método de descomposición empleado, es conveniente cuantificar su calidad. Para ello, se realiza la comparación directa entre los resultados obtenidos con la descomposición realizada con el método de dos micrófonos comentada anteriormente, y la técnica de descomposición presentada en el apartado anterior (beamforming $L C M V$ ) empleando 4 micrófonos, tal y como se ha descrito al comienzo del capítulo, introduciendo en ambas descomposiciones las correcciones de atenuación viscosa a fin de evitar esta posible fuente de error.

Se utiliza la técnica de los dos micrófonos para validar nuestro método de descomposición, por estar extensamente probada y avalada por la literatura 

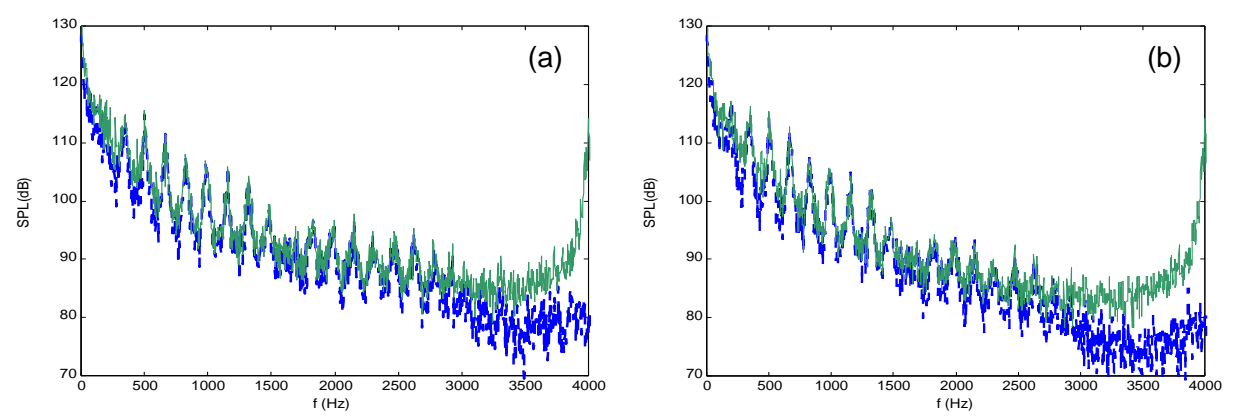

Figura 3.8: Comparación de los espectros de la señal incidente (a) y reflejada (b), obtenidas con el método de los dos micrófonos (linea continua) y el beamforming LCMV (linea discontinua).

científica $[1,26,51]$. La comparativa entre estos dos métodos de descomposición se recoge en la figura 3.8 donde se representan los espectros de la señal incidente y reflejada obtenidas con ambos métodos. En ella se observan resultados similares para ambos procedimientos, confirmando así la validez del método del beamforming como técnica de obtención de las componentes incidente y reflejada de las señales medidas. Además, pueden apreciarse relativas mejoras a alta frecuencia siendo el algoritmo de descomposición presentado (beamforming $L C M V$ ) capaz de extraer más información, lo que parece coherente con el hecho de que el efecto de las contribuciones interferentes e incorreladas de otras fuentes o del ruido suelen presentarse en dominios de alta frecuencia, ratificándose así que el método de descomposición empleado parece ser más adecuado para estos casos. También se observan diferencias a bajas frecuencias, sin embargo ninguno de los dos métodos proporciona información de señales coherentes en ese rango de frecuencias; únicamente se aprecia un reparto de la energía más o menos uniforme.

\subsection{Validación de la potencia de la técnica de esti- mación de la intensidad}

Una vez descrito y validado el método de descomposición de la onda de presión acústica en la cánula de escape de un elemento simple, en sus componentes incidente y reflejada, nos enfrentamos a la tarea de la estimación de la intensidad transmitida, valorando las diferencias y semejanzas entre el cálculo de la potencia sonora neta transmitida por la fuente interna (elemento analizado) y la medida de la potencia radiada en el campo libre. Tomaremos como 
hipótesis de partida que las diferencias espectrales que se observen pueden deberse a dos factores:

- Efectos de la descarga estacionaria del flujo, no presentes en las medidas de la fuente interna (intensimetría en conductos) y que, sin embargo, si que estarán contenidos en la medida externa. Estos fenómenos, como ya se mencionó, estarán asociados a fenómenos de vorticidad y turbulencia generados en la sección de salida del conducto.

- Componentes espectrales de la fuente interna no radiados al exterior.

Como se ha comentado se realiza una comparativa centrada en la diferencia de las potencias acústicas transmitida y radiada en base al principio de conservación de la energía acústica, con el objeto de poder analizar con mayor precisión a qué fenómenos se deben las discrepancias observadas. A continuación se detalla la obtención de estas magnitudes acústicas.

La potencia acústica medida (potencia radiada) se obtiene a través de la medida de la presión acústica a 1m de la boca de la cánula de salida en una cámara semianecoica, que como ya se comentó en el capítulo anterior permite asegurar condiciones de campo libre y la calidad de las medidas realizadas. Estas medidas de presión se realizan mediante micrófonos electrostáticos de campo libre de $\frac{1}{2}$ " de diámetro del tipo FALCON 4190, cuyas características están detalladas en el apéndice $\mathrm{C}$, recordando aquí únicamente la ventaja que presentan frente al uso de las sondas de intensidad al presentar una mejor respuesta para un rango de frecuencia más amplio, siendo su uso más adecuado para las medidas del ruido generado por un flujo estacionario debido a que su contribución es más importante para frecuencias superiores a $300 \mathrm{~Hz}$ [46].

Una vez medida la presión acústica, $p$, se estima la intensidad radiada, $I_{r}(r)$, mediante la relación $I_{r}(r)=p^{2} / \rho_{o} a_{o}$ (siendo $\rho_{o}$ y $a_{o}$ la densidad del flujo y velocidad del sonido, a temperatura ambiente), obteniendo finalmente la potencia acústica radiada $W_{r}$ empleando la ecuación (3.1), e integrando $I_{r}(r)$ sobre una semiesfera de radio $r=1 \mathrm{~m}$. De modo análogo se determina la potencia transmitida por la fuente interna $W_{t}$, empleando nuevamente la ecuación (3.1) donde se sustituye el valor de la estimación realizada de la intensidad neta transmitida, $I_{t}$, en la cánula de escape (3.18) a través del área de dicha cánula, $A_{s}$.

En las figuras 3.9, 3.10 y 3.11 se representa el nivel de intensidad radiada medida y de intensidad transmitida estimada para una geometría simple, una cámara de expansión reversa (configuración muy empleada como parte 
posterior de silenciadores comerciales), corregida esta última magnitud con la relación de áreas derivadas de la igualdad de potencias sonoras, de forma que la intensidad transmitida a una distancia $r$ de la boca de la cánula de salida, $I_{t}(r)$ viene dada por la expresión: $I_{t}(r)=I_{t} \frac{\pi a^{2}}{2 \pi r^{2}}$, donde $a$ es el radio de la cánula de salida.

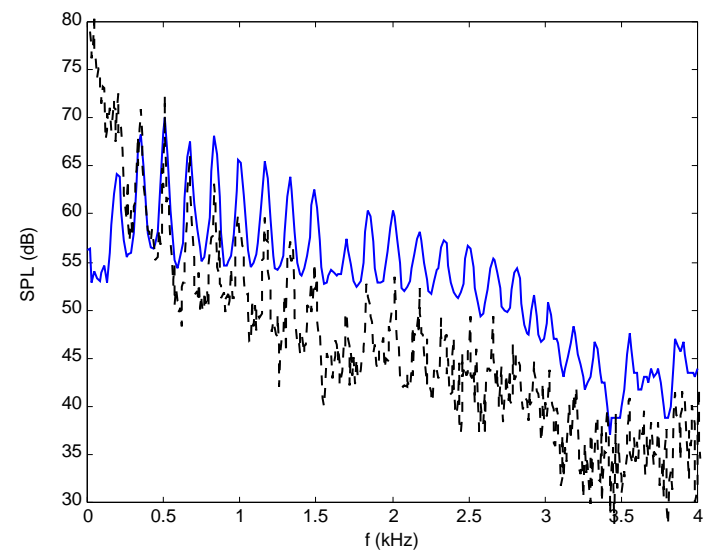

Figura 3.9: Espectro de la intensidad radiada (linea continua) y transmitida (linea discontinua), para un flujo estacionario de número de Mach $M=0.1$.

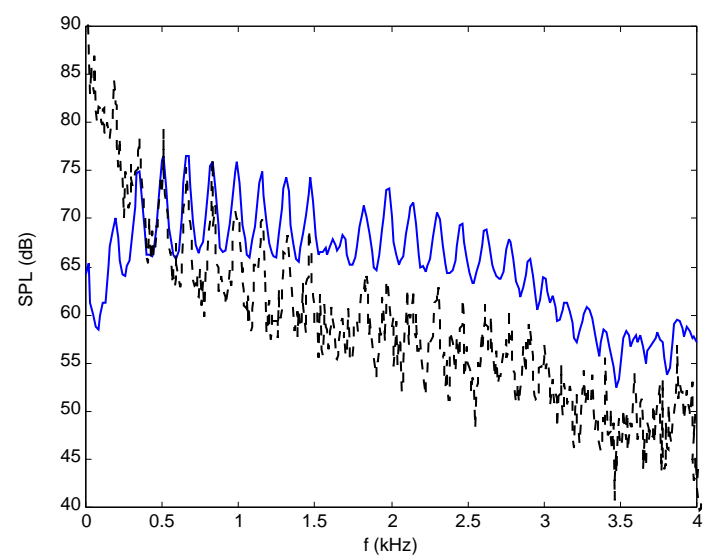

Figura 3.10: Espectro de la intensidad radiada (linea continua) y transmitida (linea discontinua), para un flujo estacionario de número de Mach $M=0.16$. 


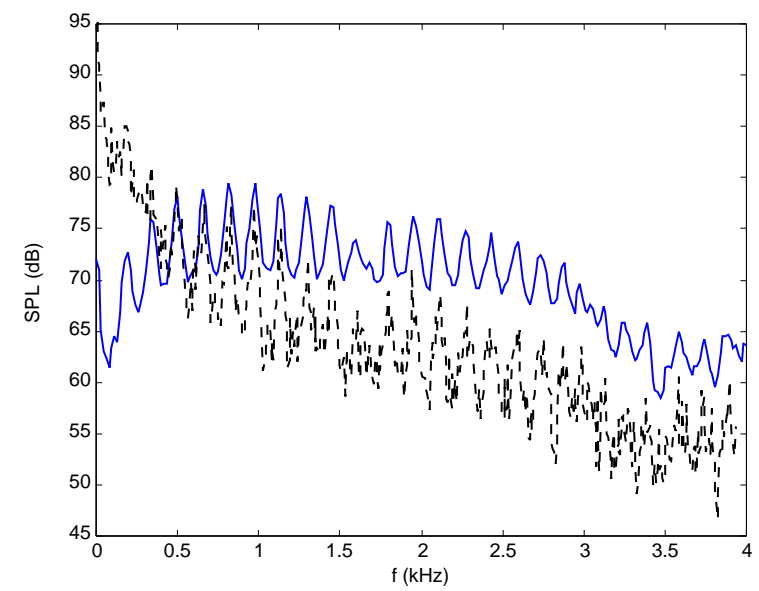

Figura 3.11: Espectro de la intensidad radiada (linea continua) y transmitida (linea discontinua), para un flujo estacionario de número de Mach $M=0.2$.

De la comparación de estas figuras (figuras de 3.9 a 3.11) se pueden extraer las siguientes conclusiones:

1. A medida que el número de Mach (velocidad del flujo) aumenta la intensidad transmitida aumenta en mayor proporción que la intensidad radiada, como se extrae de la comparación entre las figuras 3.9 y 3.11 , observándose una mejor estimación del cuarto y séptimo armónico de las frecuencias características de la cánula al aumentar la velocidad del flujo. Por otro lado también se observa un mayor valor de la intensidad transmitida para el segundo armónico de esta frecuencia en el espectro obtenido con $M=0.2$ (ver figura 3.11).

2. Se observa un mismo patrón en la evolución de ambos espectros al aumentar la velocidad del flujo, tendiendo ambos a un comportamiento más plano.

El efecto de la velocidad del flujo sobre la intensidad transmitida no radiada es justificada por los estudios de diversos autores. En primer lugar, Chung y Blaser [26] señalan que al aumentar el flujo medio uniforme aumenta el flujo neto de energía propagada a lo largo de la cánula, y ésto incrementa el flujo de energía que no es radiado acústicamente desde el extremo del conducto, ya que es absorbido por las interacciones con el campo de flujo. Por otro lado, Morfey [120] menciona las posibles limitaciones del principio de conservación 
de la energía acústica en flujos donde exista vorticidad, ya que la presencia de vorticidad en flujos estacionarios induce a que el flujo a la salida de una tobera sea débilmente inestable. Asociado a las ondas de inestabilidad existe un fenómeno de conversión de energía acústica en energía cinética, que produce como consecuencia una 'extracción' de energía desde el campo sonoro, sobre todo a bajas frecuencias [23]; efecto que presumiblemente se producirá a la salida de la cánula aunque no exista ninguna tobera en ella. Este efecto fue analizado cuantitativamente por Howe [79] para el caso de la emisión sonora para un chorro libre, a través del cociente entre intensidad radiada $\left(W_{r}\right)$ y transmitida $\left(W_{t}\right)$, cuyo valor corresponde a la siguiente expresión

$$
\frac{W_{r}}{W_{t}} \approx \frac{(k \cdot 2 a)^{2}}{(k \cdot 2 a)^{2}+16 M /\left(1+10 M^{2} / 3\right)}
$$

Factor cuya representación para diferentes números de Mach se recoge en la figura 3.12. En ella se puede observar que a medida que aumenta la frecuencia este valor es igual a cero lo que se corresponde con $W_{r}=W_{t}$, es decir, todo la intensidad transmitida en el conducto es radiada. Sin embargo, a bajas frecuencias este valor es inferior a cero lo que se corresponde $W_{r}<W_{t}$, según Howe esto se interpreta como que la potencia neta en el conducto contiene un termino de potencia acústica que es disipada por la transferencia a energía cinética del chorro.

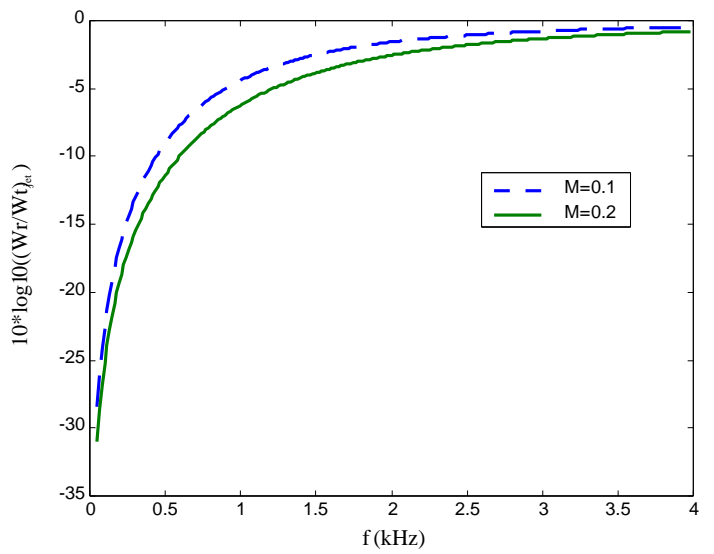

Figura 3.12: Representación del factor de absorción del sonido radiado desde un chorro libre en función de la frecuencia.

Este fenómeno presenta un acuerdo aceptable con los resultados experimentales encontrados, observándose en las figuras 3.9, 3.10 y 3.11 que la 
diferencia entre la intensidad transmitida y radiada es mayor a estas bajas frecuencias predominando la primera sobre la segunda. Además parece coherente con la forma del espectro de la intensidad radiada medida, donde se muestra una menor intensidad de los dos primeros armónicos, hecho que no es esperable en primera estancia pero que puede deberse a una mayor absorción de la energía por los fenómenos de vorticidad a estas bajas frecuencias o a que el efecto de amplificación que tiene el flujo medio sobre las frecuencias características de la cánula no sea radiado a estas frecuencias.

Por otro lado, este efecto de absorción de sonido debido a la vorticidad es más acusado al aumentar el número de Mach como se observa en la figura 3.12 , lo cual es coherente con las tendencias observadas en los espectros de la intensidad radiada (figuras de 3.9 a 3.11 ).

Con respecto al comportamiento opuesto observado a más alta frecuencia (intensidad radiada superior a la transmitida), éste parece estar asociado a los efectos de la descarga que como se ha mencionado al comienzo de esta sección no son considerados en el cálculo de la intensidad neta transmitida. Como mencionan Sheng and Meecham [158], la capa de inestabilidad generada en el borde de un conducto supone una fuente de ruido secundaria asociada a bandas de alta frecuencia, fuente de ruido que podría producir un sonido de carácter dipolar. Con el objetivo de analizar en mayor profundidad esta posible contribución del efecto de la descarga de un flujo estacionario, se estudia en el siguiente apartado la modificación del espectro transmitido al asumir un factor de emisión que dé cuenta de este fenómeno.

\subsubsection{Análisis del proceso de emisión}

Para evaluar el modelo de emisión partimos de la consideración de que las diferencias observadas entre los espectros de potencia radiada, $W_{r}$, y potencia transmitida, $W_{t}$, representados en las figuras $3.9,3.10$ y 3.11 , se deben en parte a este modelo de emisión, lo que puede expresarse a modo de ecuación como:

$$
I_{r}(r) \cdot 2 \pi r^{2} \simeq I_{t} \cdot \pi a^{2} \cdot(\text { factor de emisión })
$$

Relación que nos lleva a asumir que este factor de emisión está directamente relacionado con la diferencia logarítmica de ambos niveles de intensidad sonora, llevados al mismo punto de observación con la correspondiente corrección de áreas; o lo que es lo mismo, con el cociente entre la intensidad radiada $I_{r}(r)$ y la intensidad transmitida a esa distancia de la fuente $I_{t}(r)$. Por tanto, para tener una primera aproximación a la forma matemática de este factor se representa en la figura 3.14 el cociente anteriormente mencionado, $I_{r}(r) / I_{t}(r)$, 
en función de la frecuencia. Para esta representación se ha escogido la estimación y medidas realizadas para una geometría simple, una cámara de expansión reversa representada en la figura 3.13, variando sus parámetros geométricos más relevantes: (i) distancias entre los centros de las cánulas de entrada y de salida, consideradas desde posiciones simétricas como la de la figura (146 $\mathrm{mm}$ ) a posiciones en que el tubo de salida se encuentra centrado respecto a la sección transversal de la cámara $(73 \mathrm{~mm})$, (ii) longitudes de la cámara $(25,50$, 100, $150 \mathrm{~mm}$ ) y (iv) diámetros de entrada y de salida (51, 47, 42 y $30 \mathrm{~mm}$ ), con lo que se puede asegurar que se tiene un comportamiento global del factor de emisión relativamente independiente de la geometría interna.

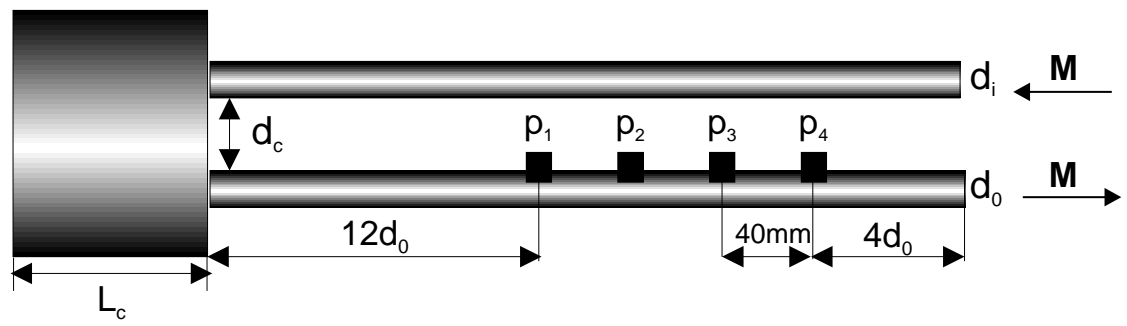

Figura 3.13: Geometría de una cámara de expansión reversa.

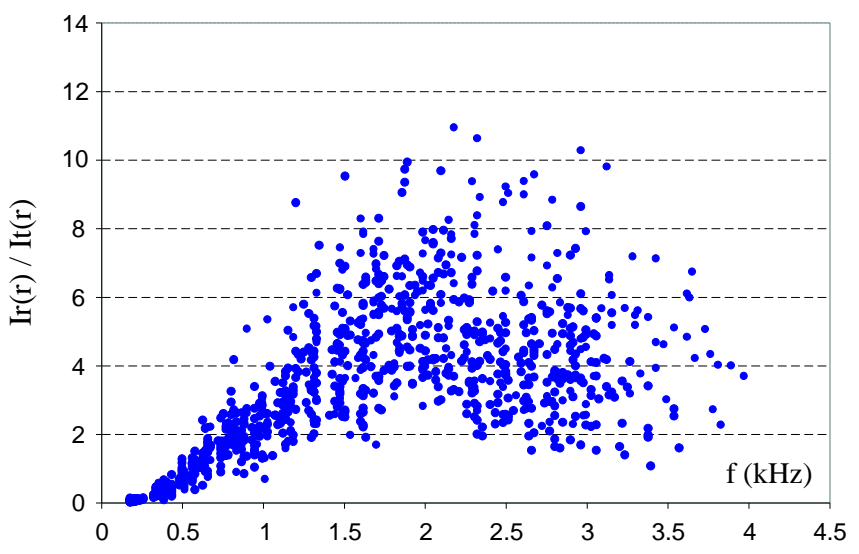

Figura 3.14: Representación del cociente entre la intensidad radiada medida y la intensidad neta transmitida (estimada) para las frecuencias características de la cánula.

Este cociente, $I_{r}(r) / I_{t}(r)$ se intenta correlacionar con algún factor simple asociado a los parámetros adimensionales que caracterizan el proceso y que como apuntan Peters et al. [137], son: el número de Mach $M=U / a_{o}$, el número de Helmholtz, $H e=k a$ y el número de Strouhal del flujo medio, $S_{t}=\omega a / U$ o lo que es lo mismo $k a / M$. 
En primer lugar se evalúa el efecto del factor de emisión encontrado de forma más extensa en la literatura [23,45], que corresponde a la consideración de que el extremo abierto radia como una fuente monopolar isótropa con una eficiencia de radiación igual al cuadrado del número de Helmholtz $(k a)^{2}$. No obstante de la figura 3.15, donde se representa el efecto de este factor frente al cociente anteriormente mencionado, se deduce que su efecto puede ser adecuado a muy bajas frecuencias pero no para el resto de frecuencias. Sin embargo, si en lugar de considerar este factor consideramos el efecto del número de Strouhal, su comportamiento parece ajustarse más a la evolución observada, tal y como nos muestra la figura 3.16. Esto puede ser consecuencia de que el número de Strouhal contiene explícitamente la velocidad media del flujo, factor que como se verá en el capítulo siguiente está íntimamente relacionado con las leyes de escalado de la intensidad sonora emitida.

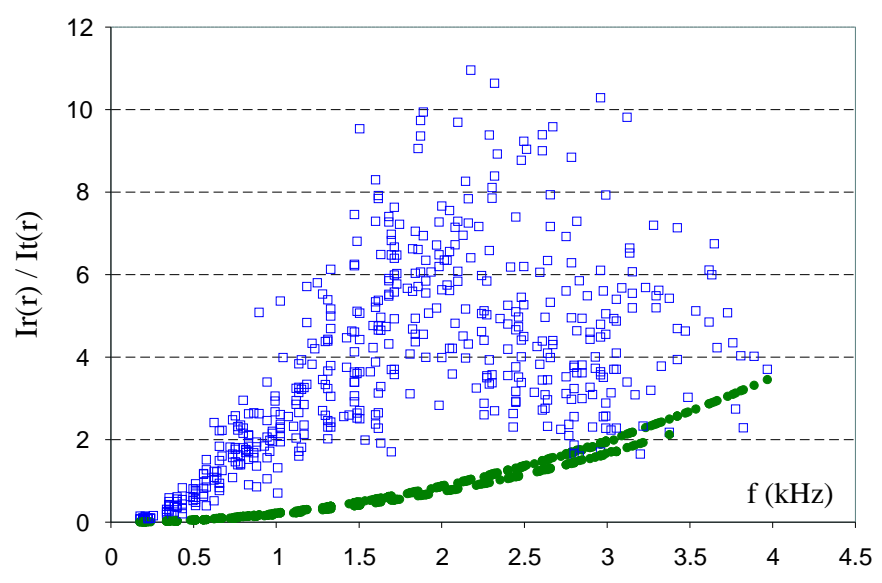

Figura 3.15: Representación del factor $(k a)^{2}(\bullet)$ frente al cociente entre la intensidad radiada medida y la intensidad neta transmitida (estimada) para las frecuencias características de la cánula.

Con el fin de validar este factor, $S_{t}$, como responsable de la eficiencia de radiación, se incluye en el modelo de intensidad emitida por la cánula (3.18) y se representa este modelo completo frente a las medidas obtenidas. En las figuras de 3.17 a 3.19 se representan los resultados obtenidos para este nuevo modelo considerando los mismos casos que en el estudio de la intensidad transmitida, con la finalidad de estudiar que mejoras introduce el factor de emisión considerado, $S_{t}$, y a su vez ver si su contribución es adecuada para diferentes velocidades del flujo o lo que es lo mismo para diferentes números de $\operatorname{Mach}(M=0.1$ a 0.2$)$.

De la comparación de estas gráficas (figuras 3.17 a 3.19) con sus corres- 
3.4. Validación de la potencia de la técnica de estimación de la intensidad111

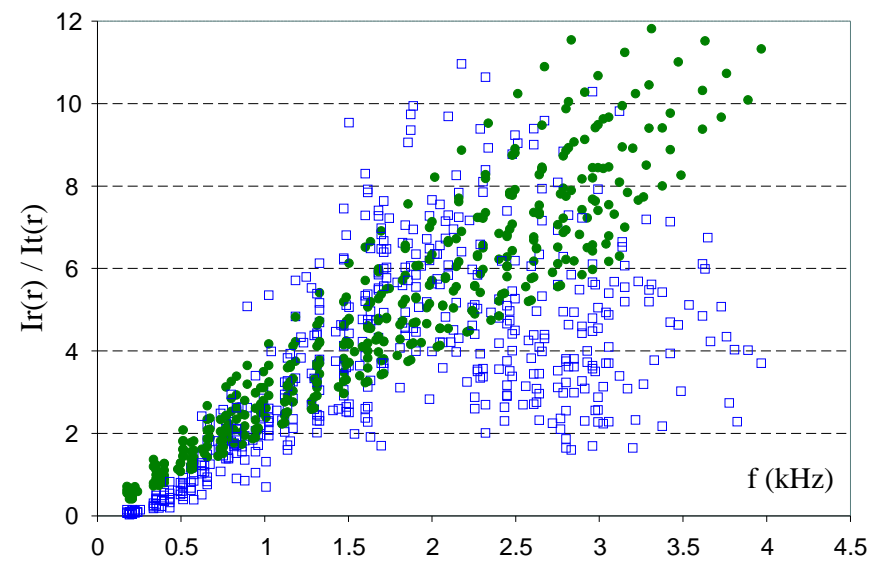

Figura 3.16: Representación del factor St (•) frente al cociente entre la intensidad radiada medida y la intensidad neta transmitida (estimada) para las frecuencias características de la cánula.

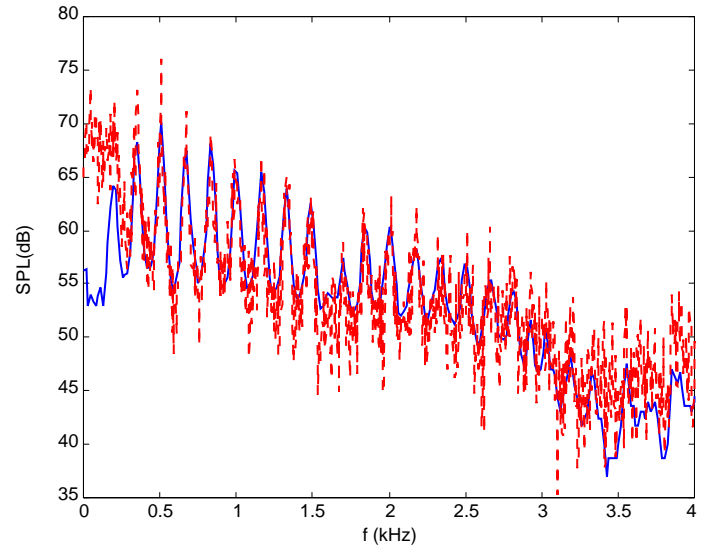

Figura 3.17: Espectro de la intensidad radiada (linea continua) y transmitida (linea discontinua) corregida con el número de Strouhal, $S_{t}$, para un flujo estacionario de número de Mach $M=0.1$. 


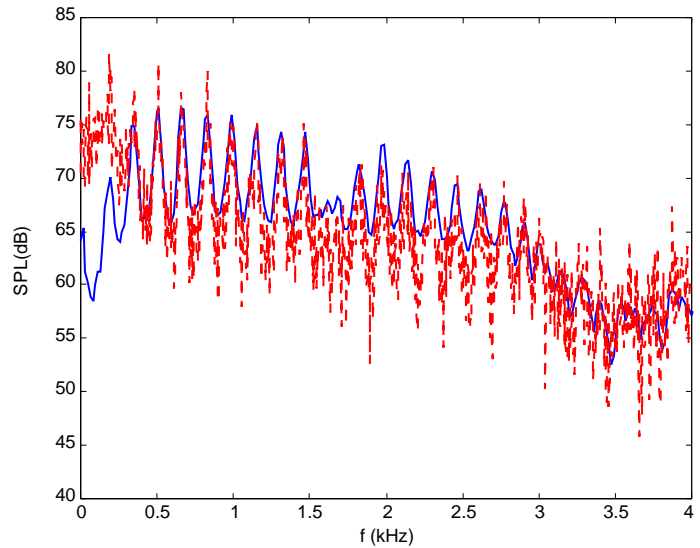

Figura 3.18: Espectro de la intensidad radiada (linea continua) y transmitida (linea discontinua) corregida con el número de Strouhal, $S_{t}$, para un flujo estacionario de número de Mach $M=0.16$.

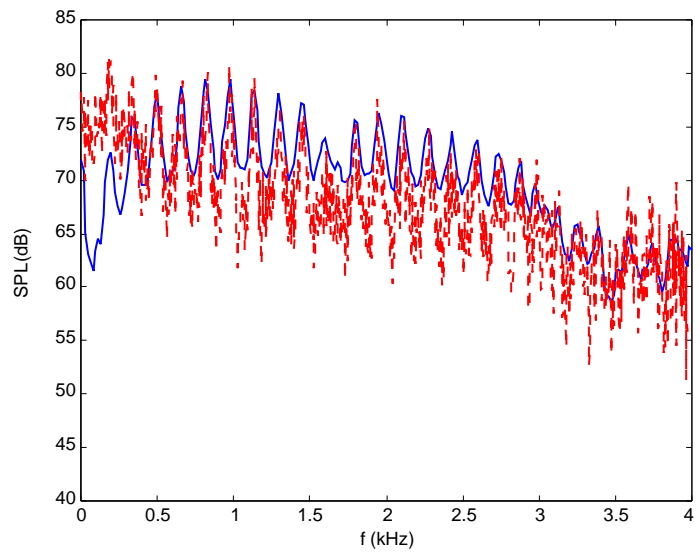

Figura 3.19: Espectro de la intensidad radiada (linea continua) y transmitida (linea discontinua) corregida con el número de Strouhal, $S_{t}$, para un flujo estacionario de número de Mach $M=0.2$. 
pondientes representaciones para la intensidad transmitida por el conducto (figuras de 3.9 a 3.11), se observa en primer lugar que este factor resulta ser adecuado para reproducir la tendencia a altas frecuencias, donde se habían observado diferencias que habíamos atribuido al fenómeno de la descarga, mientras que a bajas frecuencias sigue sin reproducirse el primer armónico correspondiente a la frecuencia característica de la cánula y al aumentar la velocidad del flujo, $M=0.2$, (figuras 3.11 y 3.19), el segundo armónico tampoco es reproducido. No obstante estos resultados permiten confirmar las hipótesis mencionadas anteriormente, es decir:

- Las sobrestimaciones observadas a bajas frecuencias se deben a que el sonido generado por la fuente interna no es radiado a tales frecuencias, además dicho flujo de energía no radiada aumenta al aumentar el flujo medio uniforme en la cánula [26] (aumento del gasto y por tanto de la velocidad media del flujo).

- Las diferencias observadas entre la intensidad transmitida y la radiada en el campo libre a más alta frecuencia pueden atribuirse a los efectos de la descarga debidos a la capa de inestabilidad que se genera en la boca del escape y que se comporta como una fuente de ruido secundaria, fenómeno que puede modelarse mediante una eficiencia de radiación igual al número de Strouhal, $S_{t}$, del flujo estacionario considerado.

En resumen, se ha observado que los efectos del ruido de flujo radiado son más importantes a partir de la segunda frecuencia característica de la cánula ( $\sim 370 \mathrm{~Hz}$ en este caso), lo que concuerda con los resultados experimentales obtenidos para los espectros del ruido de escape emitido por un motor.

Además esta técnica de intensimetría en conductos se muestra como una técnica experimental adecuada y potente para el estudio del ruido de flujo generado por elementos del sistema de escape, que presenta la cualidad de permitir analizar los mecanismos de generación interna y su relación con los espectros externos medidos, proporcionando así una guía útil no sólo para la identificación de estos mecanismos de generación sino también para introducir las debidas modificaciones en la fuente generadora del ruido de flujo, actuando sobre sus parámetros geométricos, con el fin de reducir su contribución al espectro de ruido radiado. 



\section{Capítulo 4}

\section{Análisis del comportamiento de la fuente}

4.1 Introducción $\ldots \ldots \ldots \ldots \ldots$

4.2 Evolución histórica $\ldots \ldots \ldots \ldots$

4.3 Bases teóricas sobre la generación y emisión de ruido de flujo . . . . . . . . . . . . . 119

4.3 .1 Analogía de Lighthill . . . . . . . . . . . . . . 125

4.3.1.1 Descripción y justificación de la analogía de Lighthill . . . . . . . . . . . . 125

4.3.1.2 Campo de esfuerzo externo equivalente . 127

4.3.1.3 Mecanismos de conversión de energía . . 132

4.3.1.4 Campos de radiación monopolar, dipolar y cuadripolar . . . . . . . . . . 133

4.3.1.5 Análisis dimensional de la producción de sonido aerodinámico . . . . . . . 135

4.3.1.6 Análisis crítico de la analogía de Lighthill 139

4.3.2 Métodos de resolución de la ecuación de ondas :

Función de Green . . . . . . . . . . . . . . . . . 141

4.3.3 Descomposición del campo de velocidades del fluido 143

4.4 Estudios experimentales en aeroacústica . . . . 145

4.5 Análisis experimental del ruido de flujo generado por geometrías simples . . . . . . . . . . 148

4.5.1 Análisis de las leyes de escalado . . . . . . . . . . . 162 



\subsection{Introducción}

En este capítulo se describirán los distintos procedimientos teóricos para la estimación del ruido inducido por un flujo. Se estudiarán las teorías asociadas a la cuantificación de la producción sonora, a fin de facilitar la identificación de los diferentes mecanismos físicos relacionados con el fenómeno de generación de ruido de flujo en el interior de los silenciadores. Esto ayudará a comprender los procedimientos experimentales y desarrollos teóricos utilizados para la estimación del mismo, así como la interpretación de las tendencias experimentales observadas.

En particular, los estudios sobre la generación sonora que vamos a revisar hacen referencia al análisis de emisiones globales de ruido, es decir, incluyen todos los mecanismos de generación sonora en todo el rango de frecuencia audible. Estos valores de ruido global emitido son a los que hace referencia la normativa vigente, responsables por tanto de que un sistema determinado la cumpla o no. Por ello, en este capítulo, siguiendo con la filosofía del capítulo anterior, se analizarán los resultados de ruido global obtenidos por dos vías: (1) a partir de las medidas de ruido radiado al exterior, y (2) de los resultados de las medidas de intensimetría en conductos. El objetivo es identificar el comportamiento de la fuente y derivar leyes de escalado que permitan correlacionar el ruido de flujo emitido por el sistema con sus parámetros geométricos y las condiciones del flujo. Esto proporciona algunas guías para identificar las fuentes y mecanismos generadores de ruido, analizando la importancia relativa de los diferentes parámetros geométricos involucrados en la emisión sonora, y permitiendo establecer criterios de diseño a fin de reducirla.

\subsection{Evolución histórica}

Los primeros estudios de generación de ruido aerodinámico estaban en su mayoría relacionados con la frecuencia. Estos estudios experimentales estaban dirigidos a mostrar cómo las frecuencias en el flujo eran idénticas a aquellas que se producían en el sonido, y a relacionar éstas con otras constantes del flujo. La teoría trataba de explicar cómo se generaban tales frecuencias a partir de las inestabilidades del flujo. Por entonces no se disponía de ningún procedimiento general para la estimación de la intensidad del sonido producido en términos de los detalles del flujo y no se conocía nada sobre cómo los diferentes tipos de cambios en el patrón del flujo alteraban el sonido producido.

Lighthill, el iniciador de los actuales estudios en aeroacústica, fue quien orientó la disciplina hacia el campo de la mecánica de fluidos [32]. En uno 
de sus primeros artículos [102] estudiaba el problema general asociado a la conversión de energía mecánica en energía acústica, derivado del problema de estimar el sonido radiado a partir de un flujo fluctuante. Por otra parte se centraba en la cuestión del propio flujo turbulento, en especial el campo sonoro producido por un chorro turbulento del cual disponía de datos experimentales.

Actualmente la aeroacústica está de nuevo en auge debido a la fuerte demanda que existe de ella como herramienta para resolver numerosos problemas prácticos. Entre todos estos problemas el de la reducción de ruido en sistemas de escape de motores de combustión interna está entre los de mayor interés, debido a los cada vez más restrictivos límites de emisión sonora contemplados en la normativa vigente. Existen diversas formas de reducción del ruido total emitido por el flujo de escape de un motor; en opinión de diferentes autores, entre ellos Fedorchenko [63] estas son: (i) cambiar la geometría y estructura de los elementos del sistema, cánulas, o (ii) aplicar algunos dispositivos de control activo, normalmente con el objetivo de actuar sobre los procesos no lineales de inestabilidad hidrodinámica y reducir la intensidad total de la fuente sonora. Pero para la aplicación de cualquiera de estas dos formas de actuación sobre el sistema, así como para el desarrollo de nuevas tecnologías de control, es necesario tener una comprensión teórica de los fenómenos aeroacústicos fundamentales.

De las diferentes ramas de la acústica existentes, nos centraremos en el problema particular en que la fuente acústica se encuentra emplazada en el fluido, y está por tanto asociada al ruido de flujo emitido. Este tipo de ruido es conocido como ruido turbulento, y está caracterizado por ciertos mecanismos de generación sonora asociados a las variables termofluidodinámicas del flujo. El estudio que se presenta a continuación se centrará en los mecanismos por los cuales el flujo de aire produce sonido y sobre los diferentes intentos de estimación de éste. Este fenómeno (producción de sonido por un flujo de aire) puede ser dividido en dos amplias categorías: por un lado el sonido que genera la fuente aislada del campo sonoro y por otro lado la interacción entre el sonido radiado y dicha fuente que, como sugiere Succi [161], puede constituir uno de los aspectos más relevantes. Este segundo fenómeno (interacción entre el sonido radiado y la fuente que lo genera) es el denominado ruido aerodinámico, el cuál fue definido por Curle como: el ruido generado como resultado directo del flujo de aire sin ninguna contribución debida a las vibraciones de cuerpos sólidos. 


\subsection{Bases teóricas sobre la generación y emisión de ruido de flujo}

El problema clave consiste en distinguir las procesos fuentes en el flujo de fondo y el campo acústico.

La teoría básica de la generación de ruido aerodinámico ha sido ampliamente estudiada, proponiéndose diferentes métodos para el cálculo del sonido generado para flujos subsónicos. Todas estas teorías consideran que la solución a este problema consiste en el cálculo de la propagación acústica en el espacio que rodea a la fuente hacia un punto emplazado en el campo libre, tratando de relacionar la producción sonora con las características del flujo a través de las ideas desarrolladas por Lighthill [102]. La gran ventaja y la justificación del desarrollo de su teoría radica, en que la estructura que introduce Lighthill de las fuentes emisoras de sonido tiene en cuenta explícitamente las propiedades necesarias para que se verifiquen las leyes de conservación de masa y momento en las regiones libres de contorno, introduciendo una forma de la presión radiada al campo libre susceptible de ser estimada (para el caso de flujos subsónicos $M \ll 1$ [32]) mediante leyes de escalado basadas en una sencilla representación dimensional de la fuente generadora de sonido que, como se ha comentado en la introducción, son el objetivo último de la interpretación de la emisión del ruido de flujo por silenciadores, objetivo del capítulo.

En este sentido, Lighthill asumió que era posible establecer una analogía entre el fenómeno físico real (fluctuaciones de flujo no estacionarias transfiriendo parte de su energía al campo acústico) y el problema clásico de una fuente acústica radiando en el seno de un fluido, sugiriendo la resolución de una ecuación de onda inhomogénea derivada a partir de las ecuaciones de la hidrodinámica compresible, que adquiere la siguiente forma [102]:

$$
\frac{1}{a_{o}^{2}} \cdot \frac{\partial^{2}(f(\mathbf{r}, t))}{\partial t^{2}}-\frac{\partial^{2}(f(\mathbf{r}, t))}{\partial \mathbf{r}^{2}}=q
$$

Donde $a_{o}$ denota la velocidad del sonido a temperatura ambiente, y $f(\mathbf{r}, t)$ es la función que representa el campo acústico inducido por la presencia de una fuente $q$. Este término fuente es el que describe la generación de ruido turbulento, el cual puede calcularse a partir de las variables del flujo, considerado éste incompresible y conocido, tal como muestra Möhring [118]. Dentro de esta ecuación, como es de suponer, los mayores esfuerzos se centraron, en primer lugar, en la determinación de este término fuente, siendo derivadas diferentes expresiones de $q$ que proporcionan la misma expresión de la presión acústica en campo libre al ser sustituidas en (4.1). Por otro lado, 
la solución de la ecuación (4.1) se obtiene, en general, aplicando el método de la función de Green $G(\mathbf{r}, t)$ del siguiente modo:

$$
f(\mathbf{r}, t)=\int d^{3} \mathbf{r}^{\prime} d t^{\prime} G\left(\mathbf{r}, t ; \mathbf{r}^{\prime}, t^{\prime}\right) q\left(\mathbf{r}^{\prime}, t^{\prime}\right)
$$

donde la integración se lleva a cabo sobre el volumen en el cual la fuente es distinta de cero, y siendo $t^{\prime}$ un tiempo anterior a $t$.

Por lo tanto el problema es doble: por un lado cómo relacionar la fuente $q$ con las características del flujo y por otro lado cómo determinar la función de Green de un problema dado, que conduzca a una solución para $f(\mathbf{r}, t)$ que reproduzca de forma real el comportamiento observado. En esta línea, se abordará en primer lugar la parte más relevante asociada a los mecanismos de generación sonora, forma del término fuente $q$, evaluando posteriormente el método de la función de Green para resolver la ecuación de ondas planteada (4.1), recogiéndose en el apéndice B de la presente tesis doctoral otros métodos de resolución de esta ecuación.

El término fuente $q$, se escoge en respuesta a la cuestión de cuál es el mecanismo físico real de generación de sonido aerodinámico. Éste concierne al descubrimiento del mecanismo de conversión de la energía cinética de los movimientos fluctuantes de cizalladura en energía acústica de los movimientos de fluctuación longitudinal del flujo [102].

Lighthill $[102,103]$ derivó un campo acústico a partir del sonido generado por un chorro libre turbulento, modelando el chorro (Succi [161]) como una región limitada de flujo fluctuante en el seno de un gran volumen de fluido, el cual está en reposo, y calculando la amplitud de las ondas sonoras radiadas por el flujo turbulento suponiendo que el campo sonoro era producido por una distribución estática de cuadripolos acústicos.

Curle extendió el trabajo de Lighthill sobre el chorro libre para el caso particular del tratamiento del sonido producido por la turbulencia al interaccionar con las superficies, y mostró que el ruido podía ser descrito como la suma de una integral volumétrica de cuadripolos y una integral superficial de dipolos. Powell y Meecham desarrollaron el método de las imágenes como extensión de los análisis de Lighthill y Curle, para incluir los contornos planos que no están globalmente inmersos en el flujo generador de sonido, concluyendo que la viscosidad es despreciable, y la turbulencia sobre tales superficies planas de gran extensión produce un sonido dipolar [158].

Para nuestro propósito (la comprensión de los mecanismos de generación sonora por flujos incompresibles) resulta interesante detenernos en recordar el trabajo de Tam y Auriault [164], en el que se trata el problema de la refracción 
que sufre el sonido generado por fuentes localizadas en un chorro, así como la propagación de las ondas acústicas a través del flujo medio del chorro. Para el caso particular de chorros isotermos o calientes, el efecto de esta refracción causa la deflexión de las ondas de sonido radiado fuera de la dirección del chorro. Esto produce el surgimiento de un cono de silencio en torno al eje del chorro donde se produce una reducción importante de la intensidad del sonido radiado. Estos autores defienden que dentro de un chorro, la fina escala turbulenta se distribuye aproximadamente en todo su volumen. Cada volumen local de la escala turbulenta actúa como una fuente de sonido independiente; por tanto, para calcular el sonido radiado en una dirección dada del campo lejano, es necesario realizar la suma de la contribución desde cada una de las fuentes de volumen local del chorro, como se ilustra en la figura 4.1. Esto requiere la determinación de los efectos del flujo sobre cada una de estas fuentes sonoras individuales. Para ello, se propone considerar el principio de reciprocidad en la función de Green, que se explicará posteriormente en el apartado 4.3.2.

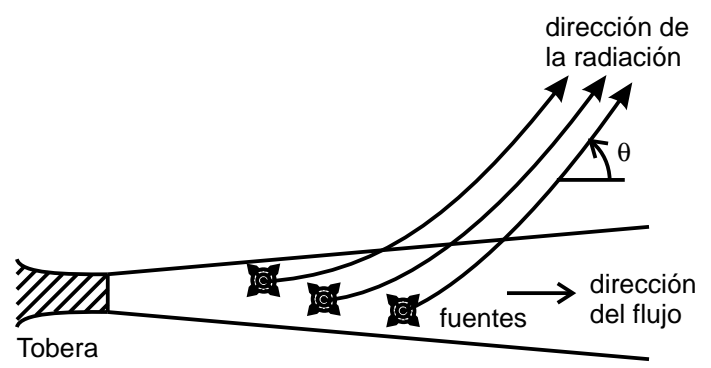

Figura 4.1: Distribución de las fuentes sonoras que contribuyen a la radiación en una dirección dada.

En relación al efecto de la refracción, Ribner [149] señala que la equivalencia entre las aproximaciones realizadas en diversas teorías se produce siempre fuera del cono de silencio, lo que permite considerar que el tratamiento de los efectos acústicos del flujo en el cono refractivo es lo que diferencia unas teorías de otras. Así Ribner sigue considerando el fluido como no viscoso, al igual que clásicamente, y también sigue interpretando el miembro de la derecha de la ecuación (4.1) como una distribución espacial de fuentes de sonido, incorporando todos los efectos del flujo (la turbulencia y flujo medio) en el término fuente, a fin de considerar los efectos de refracción.

En la figura 4.2 se puede observar una síntesis del patrón direccional del ruido de chorro, dibujando la zona refractiva: el 'cono de silencio'. 


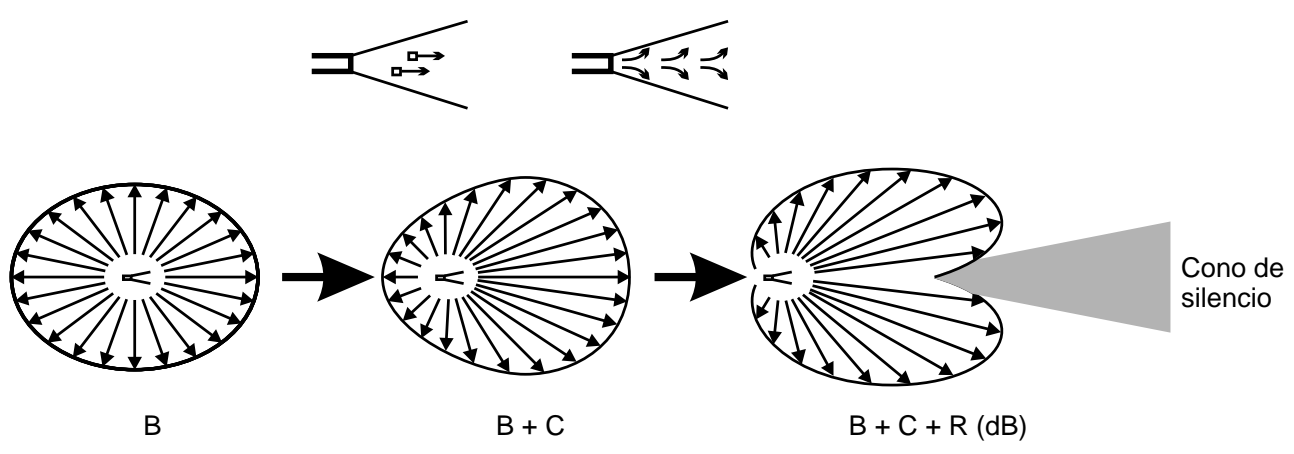

Figura 4.2: Sintensis direccional del ruido de flujo, representando la zona refractiva: el 'cono de silencio'. Escala logaritmica (decibelios). B es el patrón básico; C la amplificación convectiva; $R$ refracción.

Como ya se ha mencionado, la interpretación del término fuente fue abordada, por los diferentes autores, bajo diversos puntos de vista. A continuación se comentarán brevemente las diferentes formas de $q$ más empleadas en la actualidad, siendo analizadas y justificadas con mayor profundidad en las secciones posteriores contenidas en el presente capítulo y en el apéndice A.

Fue Lighthill (1952) [102] el primero en interpretar el término fuente $q$, bajo la consideración de que el campo sonoro corresponde a una distribución estática de cuadripolos acústicos y por tanto se expresa como una fuente cuadripolar de la forma:

$$
q=\frac{\partial^{2} T_{i j}}{\partial x_{i} \partial x_{j}}
$$

Donde $T_{i j}=\rho u_{i} u_{j}+p_{i j}-a_{o}^{2} \rho \delta_{i j}$, es el tensor de esfuerzos de Lighthill, tensión instantánea por unidad de volumen, que representa la diferencia entre el esfuerzo efectivo en el flujo real y el esfuerzo en el medio acústico uniforme en reposo. Siendo $\rho$ la densidad, $u_{i}$ las componentes de la velocidad del flujo, $p_{i j}$ el tensor de esfuerzos compresivo y $\delta_{i j}$ la delta de Kronecker. La doble divergencia en la expresión de $q$ indica el carácter cuadripolar de la fuente.

Posteriormente Ribner [118] (1962) mostró que $q$ podía ser representada como:

$$
q=-\frac{1}{a_{o}^{2}} \cdot \frac{\partial^{2} p^{(0)}}{\partial t^{2}}
$$

donde $p^{(0)}$ es la pseudo-presión sonora de Ribner, la cual verifica que:

$$
\triangle p^{(0)}=-\frac{\partial^{2} T_{i j}}{\partial x_{i} \partial x_{j}}
$$


siendo $T_{i j}$ el mismo tensor usado por Lighthill para la fuente cuadripolar. En este segundo caso la presión incompresible se comporta como una fuente de sonido monopolar.

Un tercer tipo de fuente fue derivado por Powell [139] (1964) y Howe [76] (1975), quienes establecieron que $q$ podía ser expresado como

$$
q=\rho \cdot \operatorname{div} \mathcal{L}
$$

donde $\mathcal{L}=\boldsymbol{\Omega} \times \mathbf{u}$ representa el vector de Lamb, siendo $\boldsymbol{\Omega}=\nabla \times \mathbf{u}$ el vector vorticidad, y u el campo de velocidad del fluido. Esta ecuación sugiere que la fuente debería ser expresada en términos de fuentes dipolares. Por ello, ambos autores proponen una teoría de vórtices sonoros en la que la vorticidad dentro de un contorno compacto en un medio isoentrópico débilmente compresible, es identificada como el elemento fuente.

Se puede observar en los términos fuente presentados anteriormente cómo el carácter monopolar, dipolar o cuadripolar de la fuente está relacionado con el orden de las derivadas espaciales que aparecen en las expresiones analíticas. De modo, que como afirma Howe [79] si el término fuente en la ecuación de ondas es de la forma $q=\partial^{n} F_{i j k} \ldots / \partial x_{i} \partial x_{j} \partial x_{k} \ldots$ ésta será un multipolo de orden $2^{n}$.

Además, debe mencionarse que, independientemente de la naturaleza multipolar particular de cualquier fuente, existe una única interpretación natural de la fuente en aeroacústica, independientemente de que una u otra formulación puede ser más conveniente desde un punto de vista formal. En este sentido Legendre [97] comenta que tanto la representación cuadripolar de la fuente dada por Lighthill como la representación de Ribner de una distribución de fuentes monopolares, no son contradictorias entre sí, ya que en ninguna de ellas existen singularidades dentro del fluido, simplemente se ha escogido una representación matemática distinta del proceso físico real que acontece. Además, bajo ciertas restricciones se puede pasar de una representación a otra por integración de sus diferentes partes. Una de las cualidades matemáticas más relevantes de estas representaciones es el hecho de que así como la fuente monopolar tiene una representación matemática simple y única como un campo escalar, la fuente cuadripolar es un campo tensorial, de modo que cualquier campo tensorial con la misma doble divergencia debería producir el mismo campo sonoro. Esta representación matemática de las fuentes monopolar y cuadripolar, unida a la representación vectorial de la fuente dipolar les confiere a cada una un diagrama de radiación diferente en campo libre (ver figura 4.3). 


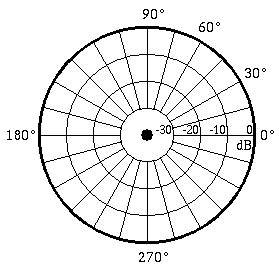

(a) monopolo

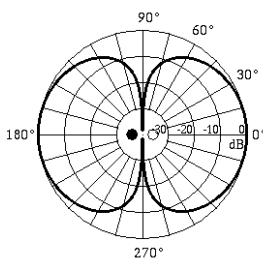

(b) dipolo

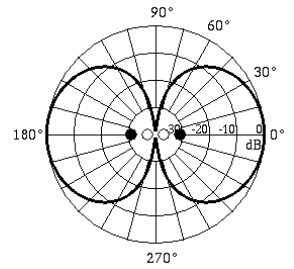

(c) cuadripolo longitudinal

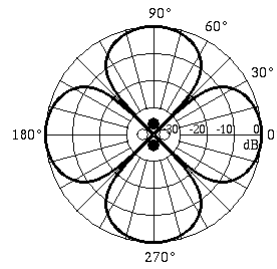

(d) cuadripolo lateral

Figura 4.3: Diagramas de radiación de la intensidad sonora en campo libre de las fuentes: (a) monopolar; (b) dipolar, (c) cuadripolar longitudinal y (d) cuadripolar lateral.

La afirmación de Möhring [118] de que los tres tipos de fuentes presentados (monopolar, dipolar y cuadripolar) conducen al mismo campo sonoro en el campo libre, está asociada al hecho de que los esfuerzos monopolar y dipolar totales de las fuentes en (4.4) y (4.6) tenderán a extinguirse fuera de la región local en que están definidos. La principal diferencia entre estas fuentes, además de su diferente forma, radica según Möhring en su grado de compacidad, entendiendo por fuente acústicamente compacta aquella que verifica que la extensión del fluido ocupado por la fuente es mucho menor que la longitud de onda de su radiación sonora [79]. Para un campo de flujo compacto la fuente sonora es aparentemente de tipo cuadripolar y lineal en la vorticidad, por lo tanto también lineal en la velocidad. Además la sensibilidad frente a la representación de los detalles finos de la fuente aumenta para determinadas representaciones del término fuente $q$, debido a los diferentes comportamientos de ésta más allá de la región de flujo donde: $T_{i j}$ decrece como $|\mathbf{r}|^{-6}$ si se consideran los valores incompresibles, $\mathcal{L}$ se anula fuera de la región que contiene la vorticidad, y $p^{(0)}$ decrece como $|\mathbf{r}|^{-3}$, como ratifica Crighton [32].

Se debe hacer mención explícita del hecho de que todos estos términos fuente son funciones espacio temporales. Físicamente, dichas fuentes aparecerán en regiones donde existan grandes tensiones de cizalla, y por tanto será muy importante conocer las variaciones temporales de la fuente para poder determinar el campo de presión.

Teniendo en cuenta que Lighthill fue el pionero de estos estudios parece coherente comenzar con la descripción de sus teorías, las cuales proporcionarán una visión global del problema de la emisión sonora por un flujo fluctuante, en particular un chorro turbulento. 


\subsubsection{Analogía de Lighthill}

La idea que "se esconde" tras la analogía de Lighthill es simple pero poderosa, el proceso completo de generación de ruido puede ser reducido matemáticamente al estudio de la propagación de ondas en un medio en reposo con el efecto del flujo reemplazado por fuentes cuadripolares [62]. Esto, como reconoce Davies [43], proporciona una guía teórica esencial para la organización de los datos recogidos en las observaciones y su posterior tratamiento ya que, como se ha comentado anteriormente, la forma de la fuente permite, en el caso de flujos subsónicos, derivar leyes de escalado que asocien este término fuente a dimensiones conocidas del sistema generador. Con este objetivo vamos a desarrollar con más profundidad la analogía acústica propuesta por Lighthill, ya que su reducción al estudio dimensional nos servirá de guía para conocer la naturaleza del ruido de flujo emitido por los silenciadores.

\subsubsection{Descripción y justificación de la analogía de Lighthill}

Lighthill consideraba que la turbulencia, o más explícitamente el flujo fluctuante, ocupa una región finita y confinada $G_{f}$, rodeada por un volumen ilimitado de fluido en reposo. De esta forma, las ecuaciones que gobiernan las fluctuaciones de densidad en el fluido podrían ser comparadas con aquellas que son apropiadas para un medio acústico uniforme en reposo, que coincide con el fluido real fuera de la región del flujo fluctuante. La diferencia entre estas dos clases de ecuaciones podría considerarse como si un campo de fuerza externa fluctuante, conocido si el flujo es conocido, estuviera actuando en el mencionado medio uniforme en reposo y por tanto radiando sonido de acuerdo con las leyes ordinarias de la acústica.

Este esquema tiene la gran ventaja de disponer de un sistema libre en el que el sonido es producido por un hipotético campo de fuerza externa. Este término fuente, se reemplaza en términos de fuerzas equivalentes, que de por sí incluyen los esfuerzos reales que generan el sonido, los esfuerzos asociados a la "back-reaction" del sonido en el flujo (esfuerzos que serían únicamente importantes en el caso de existiera en el sistema un resonador que amplifique el sonido [102]), los efectos de la convección del sonido con el flujo turbulento y la propagación de la variable velocidad dentro de éste.

Como se ha mencionado con anterioridad Lighthill [104] considera que un flujo turbulento genera el mismo campo sonoro que una cierta distribución de cuadripolos, con un esfuerzo cuadripolar por unidad de volumen conocido, siendo importante recordar que, mientras que el esfuerzo dipolar implica una dirección (esto es, el pequeño desplazamiento de la fuente negativa a la fuente 
positiva) y de acuerdo con ello es un vector, la tensión de un cuadripolo involucra dos direcciones (no sólo aquélla que va de dipolos iguales a opuestos sino también la relacionada con el desplazamiento entre ellos) y es por tanto un tensor.

La comparación con las ecuaciones que gobiernan la generación sonora para un medio acústico uniforme en reposo, se realiza más fácilmente si se escribe la ecuación de la conservación del momento en su forma original, usada sistemáticamente por Reynolds [102]. Esta ecuación adquiere la siguiente forma para un medio continuo arbitrario en ausencia de fuerzas externas

$$
\frac{\partial}{\partial t}\left(\rho u_{i}\right)+\frac{\partial}{\partial x_{j}}\left(\rho u_{i} u_{j}+p_{i j}\right)=0
$$

donde $\rho$ es la densidad, $u_{i}$ es la velocidad en la dirección $x_{i}$.

El flujo de momento $\left(\rho u_{i}\right)$ dado por la ecuación (4.7) representa una transferencia de momento debida: (i) al transporte mecánico de las distintas partículas de fluido de un lugar a otro $\left(\rho u_{i} u_{j}\right)$ y (ii) a las fuerzas de presión y posibles efectos viscosos que actúen en dicho fluido $\left(p_{i j}\right)$ [95].

Es fácil ver que el primer término $\left(\rho u_{i} u_{j}\right)$ es equivalente a un término de esfuerzo adicional denominado 'tensor de flujo de momento' o 'esfuerzo fluctuante de Reynolds' (para distinguirlo de su valor medio $\left(\overline{\rho u_{i} u_{j}}\right)$ menos el producto de sus valores medios $\overline{\rho u_{i}} \overline{u_{j}}$, que se denomina 'esfuerzo de Reynolds' en la teoría de la turbulencia); físicamente es la tasa a la cual el momento en la dirección $x_{i}$ atraviesa la unidad de superficie en la dirección $x_{j}$; su valor no es el de la tensión real ${ }^{1}$, es simplemente un tensor de flujo de momento medio para el movimiento peculiar de las moléculas.

Resumiendo, podemos decir que la ecuación del momento de Reynolds expresa que el momento en una región fija del espacio cambia a la misma razón que si el gas estuviera en reposo bajo una acción combinada de la tensión real, es decir $p_{i j}$, y la fluctuación de tensión de Reynolds $\rho u_{i} u_{j}$.

Por otro lado, en un medio acústico uniforme en reposo, el único esfuerzo posible tendrá la forma de un campo de presión hidrostática, cuyas variaciones serían proporcionales a las variaciones de densidad $\rho$, siendo la constante de proporcionalidad el cuadrado de la velocidad del sonido, $a_{o}^{2}$ [102]. Término que debería introducirse en el sistema de tensión externa considerado, de modo que las fluctuaciones de densidad en un flujo real fueran exactamente iguales a las que ocurrirían en un medio acústico uniforme sujeto a un sistema de tensión externa más general, dado por la diferencia entre la tensión efectiva en el flujo

\footnotetext{
${ }^{1}$ Esto constituye la presión hidrostática $p \delta_{i j}$ y el esfuerzo viscoso.
} 
real $\left(\rho u_{i} u_{j}+p_{i j}\right)$ y la tensión en el medio acústico uniforme en reposo $\left(a_{o}^{2} \rho\right)$. Por tanto, el tensor que marca la forma de la variación del momento contenido en la región $G_{f}$ debe adoptar la siguiente expresión

$$
T_{i j}=\rho u_{i} u_{j}+p_{i j}-a_{o}^{2} \rho \delta_{i j}
$$

Expresión (4.8) que derivaremos y analizaremos con mayor profundidad a continuación.

\subsubsection{Campo de esfuerzo externo equivalente}

La ecuación de propagación sonora en un medio uniforme, sin fuerzas de materia ni fuerzas externas, es ampliamente conocida y está gobernada por la siguiente ecuación de ondas homogénea para la densidad (Lighthill [102])

$$
\frac{\partial^{2} \rho}{\partial t^{2}}-a_{o}^{2} \cdot \nabla^{2} \rho=0
$$

Lighthill busca una ecuación de ondas de este tipo que dé cuenta del efecto de la turbulencia como generadora de sonido.

Para la derivación de las ecuaciones que gobiernan el movimiento del fluido se consideran las propiedades de compresibilidad e inercia del mismo. En este sentido, es la ecuación de continuidad la que muestra el cambio de la densidad del fluido y por tanto su compresibilidad. Su aspecto tridimensional es

$$
\frac{\partial \rho}{\partial t}+\nabla(\rho \mathbf{u})=0
$$

La ecuación de continuidad (4.10) expresa la conservación de la masa igualando el cambio de masa en una región elemental con la velocidad de salida de la masa de esta región.

La ecuación que expresa la naturaleza inercial del fluido es la ecuación de momento completa

$$
\rho \frac{\partial \mathbf{u}}{\partial t}+\rho \mathbf{u} \nabla(\mathbf{u})=\mathbf{f}
$$

donde $\mathbf{u}$ es el vector del campo velocidad y $\mathbf{f}$ es la fuerza aplicada por unidad de volumen. Ecuación que se obtiene al aplicar la segunda ley de Newton a un elemento de fluido, teniendo en cuenta que la aceleración que experimenta un elemento de fluido es

$$
\frac{\partial \mathbf{u}}{\partial t}+\mathbf{u} \nabla(\mathbf{u})
$$


En la expresión (4.12) el término lineal $\partial \mathbf{u} / \partial t$ representa la velocidad de cambio local de $\mathbf{u}$, mientras que los términos no lineales $\mathbf{u} \nabla(\mathbf{u})$ describen como cambia la velocidad de los elementos debido a su cambio de posición en el espacio. De este modo la aplicación de la segunda ley de Newton sobre pequeños elementos de fluido muestra la naturaleza inercial de éste, exigiéndose que el producto de la densidad $\rho$ por la aceleración del fluido (4.12) sea la fuerza aplicada al elemento por unidad de volumen. Esta fuerza, en ausencia de fuerzas externas se debe únicamente a los esfuerzos internos a través de los que actúan los volúmenes de fluido vecinos sobre el volumen considerado. Cuando son despreciados los efectos viscosos, esta fuerza por unidad de volumen es simplemente el gradiente de la presión del fluido $\nabla p$.

Una vez conocidas las ecuaciones de movimiento que gobiernan la propagación sonora en su forma tridimensional, pasamos a emplear la notación de subíndices con el fin de facilitar el tratamiento tensorial, esto significa tomar las coordenadas $\left(x_{1}, x_{2}, x_{3}\right)$ para el vector de posición, tal que $u_{i}$ representará la velocidad en la dirección $x_{i}$, y además cualquier sufijo repetido en un término simple será sumado para las tres componentes $(i=1, . ., 3)$. Con esta nueva notación las ecuaciones de continuidad (4.10) y conservación del momento (4.11) se reescriben como

$$
\begin{gathered}
\frac{\partial \rho}{\partial t}+\frac{\partial}{\partial x_{i}}\left(\rho u_{i}\right)=0 \\
\rho \frac{\partial u_{i}}{\partial t}+\rho u_{j} \frac{\partial u_{i}}{\partial x_{j}}+\frac{\partial p}{\partial x_{i}}=0
\end{gathered}
$$

Con el objetivo de obtener una ecuación que proporcione la velocidad de variación del momento por unidad de volumen, $\frac{\partial}{\partial t}\left(\rho u_{i}\right)$, se suma a la ecuación de conservación de momento (4.14), la ecuación de continuidad (4.13) multiplicada por $u_{i}$. Antes de realizar este último paso se intercambia el subíndice $i$ por $j$ en la ecuación de continuidad, lo que no implica perdida de generalidad por ser un índice mudo. De este modo se obtiene una nueva ecuación de conservación del momento

$$
\frac{\partial}{\partial t}\left(\rho u_{i}\right)+\frac{\partial}{\partial x_{j}}\left(\rho u_{i} u_{j}\right)+\frac{\partial p}{\partial x_{i}}=0
$$

la cual tiene la misma forma que la ecuación de continuidad, pero con diferente interpretación de los términos que aparecen. Así, la ecuación de continuidad (4.13) considera que la velocidad de cambio local de la densidad se debe a la divergencia del vector flujo de masa $\rho u_{i}$. Análogamente la ecuación (4.15) 
iguala la velocidad de cambio local de la densidad de momento con la divergencia del tensor de flujo de momento $\rho u_{i} u_{j}$ añadiendo una corrección con la fuerza de presión por unidad de volumen $-\partial p / \partial x_{i}$

En realidad los dos últimos términos de la ecuación (4.15) pueden combinarse para dar [104]

$$
\frac{\partial}{\partial t}\left(\rho u_{i}\right)+\frac{\partial}{\partial x_{i}}\left[\rho u_{i} u_{j}+\left(p-p_{o}\right) \delta_{i j}\right]=0
$$

La ecuación (4.16) incluye el efecto de que un incremento de presión $p-p_{o}$ crea un flujo de momento $\left(p-p_{o}\right) \delta_{i j}$, en el cual el factor $\delta_{i j}$ indica su isotropía, dado que este aumento de presión actúa igual en todas direcciones.

De este modo el término entre corchetes de la ecuación (4.16) es el tensor de flujo de momento total, igual a la velocidad de transporte de la componente $x_{i}$ del momento en la dirección $x_{j}$ debida: (i) a la convección por la componente $x_{j}$ de la velocidad y (ii) a la acción del aumento de presión.

Para poder expresar la ecuación (4.16) en una forma más general, incluyendo además los efectos de la transferencia de calor que vienen dados por $\nabla^{2} p-a_{o}^{2} \nabla^{2} \rho$, Lighthill [104] transforma la ecuación anterior en

$$
\frac{\partial}{\partial t}\left(\rho u_{i}\right)+a_{o}^{2} \frac{\partial \rho}{\partial x_{i}}=-\frac{\partial}{\partial x_{j}}\left\{\rho u_{i} u_{j}+\left[\left(p-p_{o}\right)-a_{o}^{2}\left(\rho-\rho_{o}\right)\right] \delta_{i j}\right\}
$$

a través de la introducción del término $\partial / \partial t\left[a_{o}^{2}\left(\rho-\rho_{o}\right)\right]$ (sumándolo y restándolo en la ecuación (4.16) y reagrupando posteriormente los términos). Finalmente, la ecuación (4.17) puede ser reescrita de una forma más compacta como

$$
\frac{\partial}{\partial t}\left(\rho u_{i}\right)+a_{o}^{2} \frac{\partial \rho}{\partial x_{i}}=-\frac{\partial T_{i j}}{\partial x_{j}}
$$

siendo

$$
T_{i j}=\rho u_{i} u_{j}+\left[\left(p-p_{o}\right)-a_{o}^{2}\left(\rho-\rho_{o}\right)\right] \delta_{i j}
$$

el llamado tensor de esfuerzos de Lighthill. Expresión que coincide con la dada por la ecuación (4.8) a falta de la contribución del esfuerzo viscoso. Si derivamos esta ecuación respecto a $x_{i}$ y sustituimos en ella el término $\partial\left(\rho u_{i}\right) / \partial x_{i}$ extraído de la ecuación de continuidad (4.13), obtenemos

$$
\frac{\partial^{2} \rho}{\partial t^{2}}-a_{o}^{2} \cdot \nabla^{2} \rho=\frac{\partial^{2} T_{i j}}{\partial x_{i} \partial x_{j}}
$$

que representa una ecuación de propagación sonora de una onda lineal en un medio uniforme en reposo debida a la aplicación de un 'término fuente' (fluctuación de tensión) ubicado en el miembro de la derecha. 
Esta transformación de las ecuaciones que gobiernan el movimiento de un fluido hace posible la interpretación de cómo el sonido es generado por flujos turbulentos, estableciendo la relación entre la dinámica de fluidos y la teoría acústica. A partir de la expresión (4.20) se interpreta que la radiación sonora generada por un fluido se debe al flujo de momento total (el término entre llaves de la ecuación (4.17) ). Por otro lado, según Howe [79], podemos interpretar esta ecuación (4.20), atendiendo a su forma, como la ecuación de ondas equivalente al cálculo del sonido aerodinámico producido por una distribución de fuentes cuadripolares cuyo esfuerzo por unidad de volumen se corresponde con el tensor de esfuerzos de Lighthill $T_{i j}$ (4.19).

Este tensor instantáneo, $T_{i j}$, aplicado en cualquier punto del fluido, puede reescribirse de una forma más general, forma adoptada por Lighthill [102], mediante la expresión (4.8), $T_{i j}=\rho u_{i} u_{j}+p_{i j}-a_{o}^{2} \rho \delta_{i j}$, donde $p_{i j}$, como ya se dijo, es el tensor de esfuerzos compresivo, que representa la fuerza en la dirección $x_{i}$ que actúa sobre una porción de fluido por unidad de superficie interior, normal a la dirección $x_{j}$.

El tensor de esfuerzos, $p_{i j}$, puede expresarse de forma general (ecuación 4.21) como la suma de un término de transferencia de impulso debida a las fuerzas de presión $\left(p \delta_{i j}\right)$ y de un término asociado a la transferencia de impulso 'viscoso' en el fluido $\left(-\sigma_{i j}\right)$, es decir, la viscosidad (rozamiento interno) que se produce por transferencia de impulso de unos puntos en que la velocidad es grande a otros puntos donde la velocidad es pequeña [95]

$$
p_{i j}=p \delta_{i j}-\sigma_{i j}
$$

donde $\sigma_{i j}$ representa el tensor de esfuerzos viscosos, tensor que depende las derivadas del campo de velocidad del siguiente modo

$$
\sigma_{i j}=\mu\left\{\frac{\partial u_{i}}{\partial x_{j}}+\frac{\partial u_{j}}{\partial x_{i}}-\frac{2}{3}\left(\frac{\partial u_{k}}{\partial x_{k}}\right) \delta_{i j}\right\}+\mu^{\prime}\left(\frac{\partial u_{k}}{\partial x_{k}}\right) \delta_{i j}
$$

donde $\mu$ representa la viscosidad dinámica $(\mu>0)$, y $\mu^{\prime}$ una viscosidad de volumen (bulk viscosity) $\left(\mu^{\prime} \geq 0\right)$. Ambos coeficientes de viscosidad son funciones de la presión y la temperatura, y en general varían a lo largo del flujo. Sin embargo, en muchos casos estas variaciones son suficientemente pequeñas, pudiendo despreciarse y considerar $\mu$ y $\mu^{\prime}$ como constantes en todo el fluido. Por otro lado, Stokes basándose en la teoría cinética de los gases supone que el coeficiente de viscosidad de volumen $\left(\mu^{\prime}\right)$ es cero, hecho que por otro lado es consistente con el estado de equilibrio. Suposición, necesaria ya que este coeficiente es difícilmente medible y además las escasos datos experimentales de que se dispone muestran que adquiere un valor muy pequeño, incluso despreciable [152]. 
En definitiva, el sistema de tensión externa $T_{i j}$ no incorpora únicamente la generación de sonido, sino también su convección con el flujo (en la parte del término $\rho u_{i} u_{j}$ ), su propagación con la variable velocidad y disipación gradual por transferencia de calor (en el término $\nabla^{2} p-a_{o}^{2} \nabla^{2} \rho$ ), así como su gradual disipación por viscosidad (en la contribución viscosa al sistema de tensión $p_{i j}$ ).

En la práctica, la disipación de la energía acústica en calor por viscosidad y por conducción, es un proceso lento; por ello, la contribución del esfuerzo viscoso en $T_{i j}$, es probablemente poco importante. Por otro lado, para flujos aproximadamente isotermos, las diferencias entre el campo de presión exacta $p \delta_{i j}$ y la aproximada $a_{o}^{2} \rho \delta_{i j}$ son igualmente poco importantes, lo que conduce a que el principal generador de sonido es el esfuerzo fluctuante de Reynolds, $\rho u_{i} u_{j}$

Landau [95] llega a la ecuación de Lighthill partiendo de la teoría lineal del sonido que afirma que el movimiento vibratorio de pequeña amplitud dentro de un fluido compresible es el que constituye las ondas sonoras, es decir, considera perturbaciones débiles en las ecuaciones de movimiento de dicho tipo de fluidos, pequeñas variaciones de presión $p^{\prime}$ y densidad $\rho^{\prime}$ frente a los valores de presión y densidad en un fluido en reposo, $\rho_{o}$ y $p_{o},\left(\rho^{\prime} \ll \rho_{o}, p^{\prime} \ll p_{o}\right)$, adoptando las ecuaciones de presión y densidad las siguientes expresiones

$$
p=p_{o}+p^{\prime} \quad y \quad \rho=\rho_{o}+\rho^{\prime}
$$

Por otro lado, este mismo autor analiza la turbulencia dentro del marco de fluidos incompresibles, suponiendo además que la velocidad del flujo turbulento es pequeña en comparación con la velocidad del sonido pero es más grande que la velocidad del fluido dentro de la onda sonora, lo que permite conservar el término no lineal $\mathbf{u} \cdot \nabla \mathbf{u}$ de la ecuación (4.14). Si además tenemos en cuenta que la onda sonora en un fluido ideal es adiabática como cualquier otro movimiento dentro del fluido, encontramos que

$$
p^{\prime}=\left(\frac{\partial p}{\partial \rho_{o}}\right)_{S} \cdot \rho^{\prime}=a_{o}^{2} \rho^{\prime}
$$

llegando a la expresión derivada por Landau [95] para la ecuación del sonido linealizada

$$
\frac{\partial^{2} \rho^{\prime}}{\partial t^{2}}-a_{o}^{2} \cdot \nabla^{2} \rho^{\prime}=\rho_{o} \frac{\partial^{2} u_{i} u_{j}}{\partial x_{i} \partial x_{j}}
$$

equivalente a la ecuación derivada por Lighthill [102] para la expresión del tensor $T_{i j}=\rho_{o} u_{i} u_{j}$, bajo las mismas consideraciones del flujo, asumiendo que el esfuerzo viscoso en $T_{i j}$ puede ser despreciado en el flujo. Además Lighthill [102] afirma que para bajos números de Mach, cualquier diferencia en la 
temperatura existente entre el flujo y el aire exterior se debe únicamente al calentamiento o enfriamiento cinético (esto es, calentando el fluido por fricción o enfriándolo por rápida aceleración), lo que hace que $T_{i j}$ sea aproximadamente igual a $\rho_{o} u_{i} u_{j}$ con un error proporcional al orden del cuadrado del número de Mach $M$.

En realidad, la solución de la ecuación de sonido, teniendo en cuenta estos efectos de viscosidad y conducción de calor ya fue dada por Kirchhoff. $\mathrm{Su}$ análisis, recogido en la Theory of Sound de Rayleigh [145], muestra que la tensión equivalente a los efectos de viscosidad y conducción de calor, causa solamente una perdida del sonido generado debido a la conversión de energía acústica en calor por este proceso, lo cual como se indicó anteriormente es despreciable excepto para fenómenos a gran escala. Por esto, fuera del flujo de aire la densidad satisface las ecuaciones normales del sonido (4.9), y las fluctuaciones de densidad causadas por la tensión efectiva aplicada dentro del flujo de aire son propagadas acústicamente.

Finalmente cabe resaltar que la formulación de Landau [95] de esta ecuación separa explícitamente un campo de fluctuaciones turbulentas incompresibles, campo de fluctuaciones hidrodinámicas, del campo de fluctuaciones compresibles, campo acústico. Lo cuál es similar a la descomposición del campo de velocidades en una parte solenoidal y una irrotacional que será discutida en la sección 4.3.3.

Por otro lado, la aproximación de $T_{i j} \approx \rho_{o} u_{i} u_{j}$, propuesta para bajos números de Mach, se relaciona directamente con la teoría de Powell y Howe [142] del sonido generado por la vorticidad, a través de la expresión del campo de velocidad del flujo incompresible $\mathbf{u}$ en términos de la vorticidad $\boldsymbol{\Omega}=\nabla \times \mathbf{u}$ usando la ley de Biot-Savart [32], como se puede comprobar en el apéndice A de la presente tesis doctoral.

\subsubsection{Mecanismos de conversión de energía}

Una vez conocida la expresión del término fuente en la analogía de Lighthill para el problema de generación de sonido aerodinámico, cabe citar los tres mecanismos de conversión de la energía cinética de los movimientos de cizalladura en energía acústica de los movimientos de fluctuación longitudinal, que pueden resumirse como sigue:

(i) Forzando una fluctuación de masa en una región fija del espacio, como con un altavoz o diafragma sumergido en un plano. 
(ii) Forzando una fluctuación de momento en una región fija del espacio, o lo que es lo mismo, forzando el flujo de gasto másico a través de una superficie fija; ambos ocurren cuando un objeto sólido vibra después de ser golpeado.

(iii) Forzando el ritmo al que el flujo de momento atraviesa superficies fijas, como cuando el sonido es generado aerodinámicamente sin movimiento de superficies sólidas.

Dentro de esta secuencia cabe destacar: primero, que cada mecanismo de conversión de energía es menos eficiente que el precedente, y segundo, que esta afirmación es más cierta cuanto menor es la frecuencia del sonido producido o, lo que es lo mismo, cuando aumenta su longitud de onda.

Desde el punto de vista matemático esto se explica porque estos tres mecanismos se asocian a fuentes de radiación monopolar, dipolar y cuadripolar respectivamente, que como se verá en la sección referente al análisis dimensional de la producción de sonido aerodinámico, guardan entre si las mismas relaciones de eficiencia de radiación en campo libre.

\subsubsection{Campos de radiación monopolar, dipolar y cuadripolar}

En definitiva el desarrollo teórico de Lighthill muestra la naturaleza cuadripolar de los chorros libres subsónicos [102], describiendo que el campo sonoro es debido a la aplicación externa de una tensión equivalente a un esfuerzo cuadripolar por unidad de volumen. Sin embargo, al emplear la aproximación de $T_{i j} \simeq \rho_{o} u_{i} u_{j}$, aunque se suponga que la principal contribución al campo de radiación se debe a una distribución de cuadripolos, puede no estar garantizado que los campos monopolares y dipolares de pequeña fluctuación sean despreciables, ya que su contribución al sonido radiado puede ser relativamente importante dependiendo de factores como el análisis de los mismos en campo próximo o campo lejano (campo libre). Un dipolo, según Lighthill, es equivalente a una fuerza que hace que el campo de esfuerzo que produce fuerzas iguales y opuestas en ambos lados del pequeño elemento de fluido sea proporcional a la tensión local.

Por otro lado, Lighthill [102] considera que el significado adecuado de la fuente cuadripolar debe entenderse como un cierto caso límite del campo de intensidad creado por cuatro fuentes; que a grandes distancias obedece la ley de la inversa del cuadrado de la distancia, y cuya amplitud incluye tanto armónicos esféricos de orden cero como de segundo orden. En oposición a la interpretación que tradicionalmente se daba a este término de 'radiación cuadripolar', que había sido empleado en dos sentidos: (1) definiendo la parte del campo de intensidad que decae como la inversa de la cuarta (y algunas 
veces sexta) potencia de la distancia desde la fuente, y (2) como el campo de amplitud expresable en términos de armónicos esféricos de segundo orden.

Tras estos preliminares, se entienden más fácilmente las propiedades de la generación de sonido por la aplicación de tensiones fluctuantes $T_{i j}$. Tal y como muestra la ecuación (4.18), la tensión produce una fuerza por unidad de volumen igual al flujo admitido $-\partial T_{i j} / \partial x_{j}$. Por tanto, estas tensiones generan sonido como un campo dipolar de tensión $-\partial T_{i j} / \partial x_{j}$ por unidad de volumen; en particular es la expresión de la 'fuerza efectiva' que en la ecuación del momento (4.18) expresaba qué gradientes del flujo de momento total atravesaban una región elemental, produciendo cambios de momento debido a las desigualdades de flujo dentro y fuera de éste. Además el término $-\partial T_{i 1} / \partial x_{1}$ es equivalente en el límite cuando $\epsilon \rightarrow 0$ (pequeño desplazamiento) a un campo dipolar de esfuerzo por unidad de volumen

$$
\left[T_{i 1}\left(x_{1}, x_{2}, x_{3}\right)-T_{i 1}\left(x_{1}+\epsilon, x_{2}, x_{3}\right)\right] / \epsilon
$$

que es a su vez equivalente a una distribución de cuadripolos por unidad de volumen (Lightghill [104]), donde cada uno de los términos cuadripolares asociados al mismo se interpretan como un dipolo de vector de esfuerzos $\epsilon^{-1} T_{i 1}$ en $\left(x_{1}, x_{2}, x_{3}\right)$ más un segundo campo dipolar de esfuerzo $-\epsilon^{-1} T_{i 1}$ en $\left(x_{1}+\epsilon, x_{2}, x_{3}\right)$. De esta forma cualquier valor de $\epsilon^{-1} T_{i 1}\left(x_{1}, x_{2}, x_{3}\right)$ ocurre para un valor de signo positivo en $\left(x_{1}, x_{2}, x_{3}\right)$ y signo negativo en $\left(x_{1}+\epsilon, x_{2}, x_{3}\right)$. Los dos juntos, en el límite, puede decirse que constituyen un cuadripolo cuya tensión es la magnitud del vector $T_{i 1}$, y cuyos ejes están en la dirección de $T_{i 1}$ y en la dirección $x_{1}$. El resto de elementos cuadripolares $T_{i j}$, para $j=2,3$ serían interpretados de la misma forma.

Esta definición muestra que en el esfuerzo cuadripolar están incluidas dos direcciones, de acuerdo con la forma tensorial $T_{i j}$, observándose que cualquier elemento diagonal del tensor, de la forma $T_{i i}$ corresponde a una configuración alineada de dipolos, como se muestra en la parte de la izquierda de la figura (4.4) para el caso particular $T_{11}$. Este tipo de cuadripolo es comúnmente denominado 'cuadripolo longitudinal', mientras que los elementos fuera de la diagonal $T_{i j}$ (cuando $i \neq j$ ), por ejemplo $T_{12}$ representado en la parte de la derecha de la figura (4.4), corresponden a dipolos separados en una dirección perpendicular, y son llamados 'cuadripolos laterales'. Generalmente, si un cuadripolo está formado por dipolos iguales y opuestos con ejes en una dirección, cuya posición relativa está en otra dirección, estas dos direcciones son llamadas los ejes del cuadripolo. Esto permite afirmar, más concretamente, que cuando coinciden los ejes los cuadripolos pueden llamarse longitudinales, y cuando son perpendiculares se llamarán laterales. 


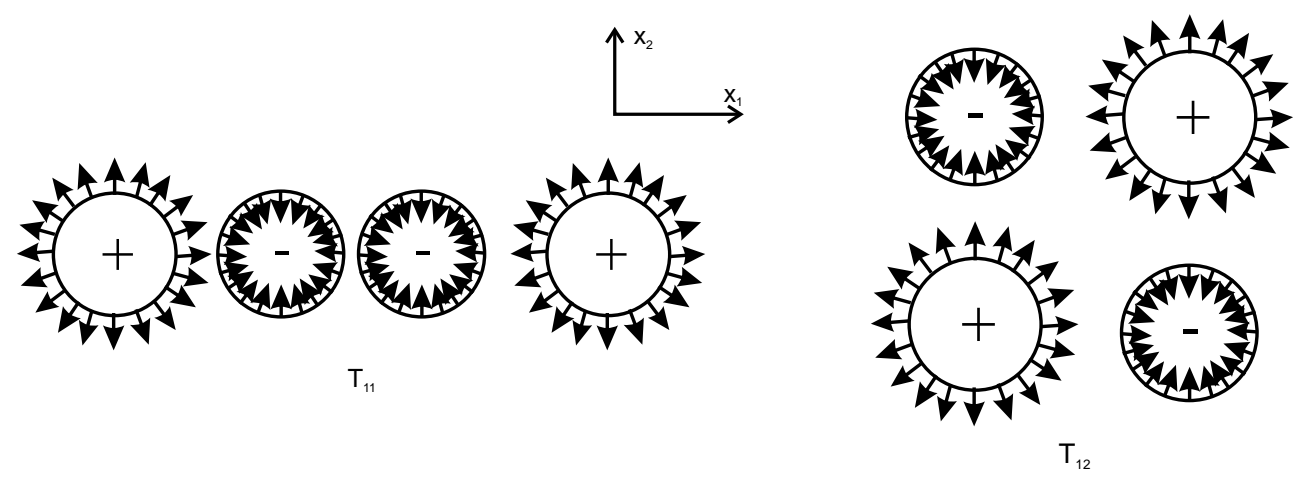

Figura 4.4: En la figura se representan a la izquierda, distribución de cuadripolos longitudinales (configuaración alineada de dipolos), correspondiente a las componentes diagonales del tensor; y a la derecha, distribución de cuadripolos laterales (dipolos separados en direcciones perpendiculares), correspondiente a las componentes no diagonales del tensor.

A este respecto, Lighthill afirma [102] que es esencial que el sonido radiado no sea estimado desde el orden de magnitud de una tensión dipolar por unidad de volumen, ya que en cualquier instante la tensión local dipolar puede anularse sin que la tensión efectiva en su entorno se anule. En ese sentido una descripción en términos cuadripolares es más realista que en términos de fuentes monopolares (escalar) o dipolares (vectorial) debido a las ventajas que le confiere su formulación matemática.

\subsubsection{Análisis dimensional de la producción de sonido aerodinámico}

Desde el punto de vista práctico, y como ya hemos apuntado, una de las aplicaciones más importantes de la determinación del término fuente en el proceso de generación sonora, es la posibilidad de identificar el tipo de fuente presente en un proceso experimental a través de una ley de escalado del nivel global de ruido radiado con la velocidad del flujo. Esta ley de escalado se obtiene a través de un análisis dimensional del orden de magnitud de la intensidad radiada, la cual se calcula de forma sencilla al resolver la ecuación de ondas general (4.1) del siguiente modo. En primer lugar la función de Green para el campo libre conduce directamente a una solución con el potencial retardado de la forma

$$
f(\mathbf{r}, t)=\frac{1}{4 \pi} \int d^{3} \mathbf{r}^{\prime} \frac{1}{\left|\mathbf{r}-\mathbf{r}^{\prime}\right|} q\left(\mathbf{r}^{\prime}, t-\frac{\left|\mathbf{r}-\mathbf{r}^{\prime}\right|}{a_{o}}\right)
$$


Expresión muy sensible a la dependencia de la fuente $q$ con el tiempo retardado $t-\left|\mathbf{r}-\mathbf{r}^{\prime}\right| / a_{o}$, donde $\left|\mathbf{r}-\mathbf{r}^{\prime}\right|$ representa la distancia desde la fuente situada en $\mathbf{r}^{\prime} \equiv\left(x_{i}^{\prime}\right)_{i=1, \ldots, 3}$ al punto de recepción en $\mathbf{r} \equiv\left(x_{i}\right)_{i=1, \ldots, 3}$. La forma de Lighthill, con su estructura cuadripolar permite obtener una expresión de la radiación en campo libre a través de los siguientes argumentos. En primer lugar, si introducimos la expresión de la fuente derivada por Lighthill en la ecuación (4.26) obtenemos

$$
p(\mathbf{r}, t)=\frac{1}{4 \pi} \frac{\partial^{2}}{\partial x_{i} \partial x_{j}} \int T_{i j}\left(\mathbf{r}^{\prime}, t-\frac{\left|\mathbf{r}-\mathbf{r}^{\prime}\right|}{a_{o}}\right) \frac{d^{3} \mathbf{r}^{\prime}}{\left|\mathbf{r}-\mathbf{r}^{\prime}\right|}
$$

Introduciendo ahora la doble derivación en la integral, se puede observar que la derivación correspondiente al denominador $\left|\mathbf{r}-\mathbf{r}^{\prime}\right|$ decae con la inversa del cubo de la distancia al punto de observación, mientras que la parte debida a la diferenciación de $T_{i j}$ cae solamente con la inversa de la primera potencia de la distancia. Por lo tanto, para puntos alejados de la fuente, el campo de fluctuación de presión está dominado por este segundo término, quedando la ecuación (4.27) reducida a

$$
p(\mathbf{r}, t) \approx \frac{1}{4 \pi} \int \frac{1}{\left|\mathbf{r}-\mathbf{r}^{\prime}\right|} \frac{\partial^{2}}{\partial x_{i} \partial x_{j}} T_{i j}\left(\mathbf{r}^{\prime}, t-\frac{\left|\mathbf{r}-\mathbf{r}^{\prime}\right|}{a_{o}}\right) d^{3} \mathbf{r}^{\prime}
$$

Por otro lado si tenemos en cuenta la definición matemática de la diferencial de una función que depende de dos variables

$$
\frac{\partial f(a, b)}{\partial x}=\frac{\partial f}{\partial a} \frac{\partial a}{\partial x}+\frac{\partial f}{\partial b} \frac{\partial b}{\partial x}
$$

y la aplicamos a nuestra función $T_{i j}\left(\mathbf{r}^{\prime}, t-\left|\mathbf{r}-\mathbf{r}^{\prime}\right| / a_{o}\right)$ en su derivación respecto de $x_{i}$ obtenemos la siguiente igualdad

$$
\frac{\partial T_{i j}\left(\mathbf{r}^{\prime}, t-\frac{\left|\mathbf{r}-\mathbf{r}^{\prime}\right|}{a_{o}}\right)}{\partial x_{i}}=\frac{\partial T_{i j}}{\partial t} \frac{\partial\left(t-\left|\mathbf{r}-\mathbf{r}^{\prime}\right| / a_{o}\right)}{\partial x_{i}}=-\frac{1}{a_{o}} \frac{\partial\left|\mathbf{r}-\mathbf{r}^{\prime}\right|}{\partial x_{i}} \frac{\partial T_{i j}}{\partial t}
$$

Ambas ecuaciones (4.28) y (4.30), permiten obtener una solución para la fluctuación de presión en el campo libre (en puntos suficientemente alejados del flujo) de la forma

$$
p(r, t)=\frac{1}{4 \pi a_{o}^{2}} \cdot \int \frac{\left(x_{i}-x_{i}^{\prime}\right)\left(x_{j}-x_{j}^{\prime}\right)}{\left|\mathbf{r}-\mathbf{r}^{\prime}\right|^{3}} \frac{\partial}{\partial t^{2}} T_{i j}\left(\mathbf{r}^{\prime}, t-\frac{\left|\mathbf{r}-\mathbf{r}^{\prime}\right|}{a_{o}}\right) d^{3} r^{\prime}
$$

Para el campo de sonido radiado, esta expresión debe dar un resultado exacto para la energía total radiada y su distribución direccional, ya que 
únicamente se excluyen los términos con decaimiento superior a la inversa de la primera potencia de la distancia. Razón por la que a distancias suficientemente grandes comparadas con las dimensiones del flujo se puede realizar la aproximación de $x_{i}-x_{i}^{\prime}$ por $x_{i}$ en (4.31) sin despreciar ningún término del orden $|\mathbf{r}|^{-1}$, con tal de que el origen se tome en el interior del flujo. Esto proporciona una forma aún más simple para el campo de radiación

$$
p(r, t)=\frac{1}{4 \pi a_{o}^{2}} \frac{x_{i} x_{j}}{|\mathbf{r}|^{3}} \frac{\partial}{\partial t^{2}} \int T_{i j}\left(\mathbf{r}^{\prime}, t-\frac{\left|\mathbf{r}-\mathbf{r}^{\prime}\right|}{a_{o}}\right) d^{3} r^{\prime}
$$

que para bajos números de Mach, es independiente de las variaciones en el tiempo retardado.

A partir de la ecuación (4.32), Lighthill [102] realiza un análisis dimensional del orden de magnitud de la intensidad radiada en función de los parámetros característicos del flujo turbulento. En este análisis se considera que el torbellino tiene una longitud de escala $\ell$ y una velocidad característica $U$, y por tanto la máxima diferencia del tiempo retardado $\left(t-\left|\mathbf{r}-\mathbf{r}^{\prime}\right| / a_{o}\right)$ es del orden de $\ell / a_{o}$, un tiempo muy corto a bajos números de Mach, de forma que la escala temporal sobre la que cambia (ver ecuación (4.32)) puede, normalmente, ser evaluada en flujos turbulentos (Crighton [32]). Así puede verse que las componentes del tensor $T_{i j} \sim \rho_{o} U^{2}$, donde $U$ es la velocidad media del flujo turbulento, en cada nueva derivación temporal su orden de magnitud se encuentra multiplicado por la frecuencia característica $U / \ell$. En consecuencia la potencia radiada $W_{r}$ es

$$
W_{r} \sim \rho_{o} V U^{8} /\left(a_{o}^{5} \ell\right)
$$

donde, como se ha mencionado, $\ell$ es la longitud de escala característica de las estructuras de turbulencia (se entiende que las velocidades de pulsación de la turbulencia en diferentes puntos se extienden a unas distancias de $\sim \ell$ ), y $V$ es el volumen que define la región turbulenta. Obteniéndose la conocida ley de escalado del ruido de flujo con la octava potencia de la velocidad del flujo.

De modo similar puede mostrarse que la potencia disipada en el flujo turbulento es

$$
W_{d} \sim \rho_{o} V U^{3} / \ell
$$

así que la razón entre la potencia radiada y disipada, puede interpretarse como la eficiencia de radiación de la fuente cuadripolar, siendo esta proporcional a $M^{5}\left(M=U / a_{o}\right)$. Esto sugiere que puede reescribirse la ecuación (4.33) de la siguiente forma

$$
W_{r} \sim\left(\rho_{o} U^{3} \ell^{2}\right) \cdot\left(V / \ell^{3}\right) \cdot M^{5}
$$


la cual admite una interpretación sencilla: el primer término puede escribirse como $\left(\rho_{o} U^{2}\right) \ell^{3}(U / \ell)$, que representa la razón de abastecimiento de energía cinética $\left(\rho_{o} U^{2}\right)$ a un torbellino de escala $\ell$ a fin de mantener su movimiento a velocidad $U$, y el segundo término es el número de torbellinos de volumen $\ell^{3}$ distribuidos a través del volumen $V$. Y por tanto la expresión (4.35) es equivalente, según Crighton [32], a asumir un cierto número de remolinos no correlacionados radiando con una eficiencia proporcional a $M^{5}$.

De modo análogo se pueden deducir las correspondientes leyes de escalado con la velocidad del flujo para una fuente dipolar y monopolar a través de la consideración de la eficiencia de radiación de estas fuentes, como ya se mencionó en secciones anteriores. De acuerdo con esto, se observa que la eficiencia de radiación que presenta una fuente dipolar es mayor que la de la fuente cuadripolar, ya que para la fuente dipolar viene dada por $M^{3}$

$$
W_{r} \sim\left(\rho_{o} U^{3} \ell^{2}\right) \cdot\left(V / \ell^{3}\right) \cdot M^{3}
$$

proporcionando una ley de escalado global con $U^{6}$ para el tipo de radiación dipolar. En el caso de una fuente monopolar, la eficiencia de radiación es simplemente $M$, lo cual da una ley de escalado de la potencia radiada con $U^{4}$.

$$
W_{r} \sim\left(\rho_{o} U^{3} \ell^{2}\right) \cdot\left(V / \ell^{3}\right) \cdot M
$$

De esta forma puede observarse como el sonido aerodinámico cuadripolar difiere por un factor $M^{2}$ desde la emisión dipolar, y esta a su vez difiere también en un factor $M^{2}$ desde la potencia sonora emitida por un monopolo. Por tanto, para un flujo subsónico $(M<1)$ estas fuentes cuadripolar y dipolar se constituyen como fuentes de menor eficiencia sonora que la fuente monopolar, como recogen Howe y Bauman [77], efecto que fue verificado en el capítulo 2, en el que se realizó el análisis del ruido emitido durante el proceso de escape atribuyéndose el comportamiento monopolar a la componente pulsante del ruido y las fuentes dipolares y cuadripolares al efecto del ruido inducido por el flujo medio.

Por otro lado, como comenta Lighthill [104], el campo lejano debido a la radiación cuadripolar es mucho más débil en relación con su campo próximo que el correspondiente a una radiación dipolar. Esto significa que en algunos casos este campo lejano es apenas perceptible y en otros, cuando el esfuerzo cuadripolar es muy grande y las contribuciones de las radiaciones monopolar y dipolar son poco significativas, la radiación cuadripolar puede producir un campo sonoro extremadamente desagradable, por lo que el estudio cuantitativo de este tipo de radiación es muy interesante con el fin de poder abordar su disminución. 
Estas leyes de escalado indicarán, en casos realistas en los que varios mecanismos aparecerán juntos, cuales de todos ellos constituyen la contribución principal al ruido emitido. Por supuesto, la identificación de una ley de escalado dada indicará únicamente el hecho de que la fuente resultante a la salida del sistema analizado posee dicha naturaleza, ya que las leyes de escalado obtenidas se sustentan únicamente para emisiones en campo libre y fuentes compactas. Sin embargo, parece intuitivamente claro que a las frecuencias para las cuales la longitud de onda es mucho mayor que las dimensiones del conducto, la potencia radiada por una fuente de las dimensiones del conducto será aproximadamente la misma que la radiación generada por una fuente puntual en el campo libre, como se confirma por los resultados de Nelson y Morfey [127].

Por otro lado Lighthill [105], aplicando su teoría a casos particulares, afirma que para cuerpos que no sean necesariamente compactos existe un cálculo más refinado, empleando funciones de Green para espacios delimitados internamente más que para el espacio libre, que conducen en general a la misma conclusión: radiación cuadripolar con la dependencia en la octava potencia de $U$, encontrándose una importante excepción a esta regla para los cuerpos de puntas y aristas finas, donde los rasgos de la función de Green implican una dependencia de la radiación de la turbulencia con la quinta potencia de la velocidad del flujo [105].

\subsubsection{Análisis crítico de la analogía de Lighthill}

De acuerdo con los comentarios realizados por Fedorchenko [63] en relación a los puntos débiles de la teoría de Lighthill descrita anteriormente, podemos destacar las siguientes críticas. En primer lugar en relación a la aproximación realizada por Lighthill en su ecuación de propagación sonora de acuerdo con la expresión

$$
\frac{\partial^{2} \rho}{\partial t^{2}}-a_{o}^{2} \cdot \nabla^{2} \rho=\frac{\partial^{2} T_{i j}}{\partial x_{i} \partial x_{j}}
$$

Se observa que no existe ningún punto donde se consideren las variables del campo $\{\mathbf{u}, p, \rho\}$ separadamente, sino que se incluyen como un juego completo de variables totales desconocidas, siendo imposible determinar cualquiera de estas variables antes de obtener una solución exacta de la ecuación de ondas, ya que uno no puede analizar las propiedades características de una ecuación escalar no homogénea de tipo hiperbólico en el espacio y en el tiempo separada con varias variables. Además, esta ecuación no proporciona ninguna ayuda para separar las perturbaciones acústicas del flujo de fondo no estacionario. 
Probablemente, si se tiene éxito derivando una ecuación de alto orden autocerrada para una variable escalar, igual a la de Lighthill, incluso luego sería imposible analizar el fenómeno acústico sin separar la componente sonora de la variable que represente el campo acústico.

Respecto a esta crítica y en defensa de la ecuación derivada por Lighthill habría que añadir los resultados de Landau [95], mostrados en el apartado 4.3.1.2, al proponer la separación de la componente acústica (fluctuaciones compresibles) y las componentes turbulentas (fluctuaciones incompresibles), llegando a obtener bajo estas consideraciones una ecuación inhomogénea de segundo orden igual a la de Lighthill.

Una segunda limitación de esta analogía es la compacidad de la región considerada $G_{f}[76]$ (con tamaño $\ll \lambda$, donde $\lambda$ es la longitud de onda del sonido) que contiene flujo medio inestable no uniforme, y que está rodeada por un medio de gas homogéneo de extensión infinita con velocidad media cero y valores constantes $a_{o}, p_{o}, \rho_{o}$, en un esfuerzo por hacer la teoría más verosímil, a pesar de que una configuración tan ideal del flujo está lejos de la realidad en la mayoría de los flujos bajo estudio. Claramente, fuera de la región $G_{f}$, la ecuación (4.38) con $q=0$ puede utilizarse para describir pequeñas perturbaciones acústicas en un medio en reposo, pero este hecho es incapaz de probar que justo en $G_{f}$ todo el miembro de la derecha de la ecuación (4.38) puede interpretarse como un término fuente responsable de la generación de sonido por el flujo. Generalmente, parece absurdo exigir la compacidad de un cierto dominio del flujo (lo cual no es factible dentro de todos los posibles valores de $\lambda$, mucho menos si uno no conoce por adelantado el espectro de sonido generado) como una condición necesaria de la validez del modelo si este modelo aspira a ser general. Esta condición puede ser útil únicamente cuando uno trata de calcular la tensión promedio de la fuente sonora en $G_{f}$ tras haber obtenido la distribución de aquellas fuentes sobre todo $G_{f}$.

No obstante, en la actualidad el modelo de Lighthill continua teniendo muchos defensores, entre ellos personas influyentes en el mundo de la aeroacústica. Así, por ejemplo Crighton [32], dice que 'la teoría de Lighthill ha sido a distancia la más exitosa y versátil'; de modo similar Ffowcs Williams [64] comenta que 'la teoría de Lighthill fue una obra maestra'. En esta misma línea Crow [63] menciona, a modo de apoyo a dicha teoría, que ninguna de las descripciones físicas alternativas de modelos de fuentes sonoras aerodinámicas ha sido capaz de competir con la teoría de Lighthill. En este sentido, se puede considerar que este modelo proporciona una descripción válida del problema del sonido generado por un flujo incompresible. El irresistible atractivo de esta aproximación se explica por la aparente simplicidad que 
presenta la ecuación (4.38), obtenida para el caso particular de flujo homoentrópico subsónico (con números de Mach $M \ll 1$ ), en los cuales $p^{\prime}=p-p_{o}$ y $\rho^{\prime}=\rho-\rho_{o}$ son definidas como pequeñas perturbaciones en un flujo medio uniforme, pudiéndose escribir $p^{\prime}=p-p_{o} \approx a_{o}^{2}\left(\rho-\rho_{o}\right)$ para el caso adiabático.

\subsubsection{Métodos de resolución de la ecuación de ondas : Función de Green}

Como ya se comentó brevemente al comienzo de esta sección el problema de la determinación del campo acústico radiado por un flujo turbulento no sólo lo constituye el conocimiento del término fuente que lo genera, sino que es también necesario disponer de métodos de resolución de la ecuación de ondas inhomogénea planteada. En este apartado se aborda brevemente la descripción de los aspectos más relevantes del método de la función de Green, ya que es éste el empleado más frecuentemente en la resolución de esta ecuación de ondas (4.2), además de ser el escogido en el caso que nos ocupa. Sin embargo, otros métodos de resolución existentes en la literatura científica están recogidos en el apéndice $B$ de la presente tesis doctoral, con el fin de no sobrecargar esta sección pero sin descuidar la presentación de métodos de resolución alternativos.

Como se observa en la ecuación (4.2) el conocimiento de la función de Green $G(\mathbf{r}, t)$, de cualquier proceso de generación de ruido aerodinámico permite determinar directamente la función que representa el campo acústico. La forma de la función de Green ha sido analizada y tratada ampliamente en la literatura científica, por lo que a continuación únicamente será definida y comentadas algunas de sus propiedades, a fin de proporcionar una imagen más clara de la potencia del método. A su vez se citarán algunos de los ejemplos de aplicación más relevantes con el fin de aportar una idea global de la misma.

La función de Green en el dominio frecuencia, $G\left(\mathbf{r}, \mathbf{r}^{\prime} ; \omega\right)$, se define como la solución a la ecuación de Helmholtz inhomogénea $\left(\left[\nabla^{2}+k^{2}\right] f(\mathbf{r}, \omega)=q\right)$ cuando el término fuente es: $\delta\left(\mathbf{r}-\mathbf{r}^{\prime}\right)$; fuente puntual emplazada en $\mathbf{r}^{\prime}$ que verifica la igualdad [79]

$$
\left(\nabla^{2}+k^{2}\right) G\left(\mathbf{r}, \mathbf{r}^{\prime} ; \omega\right)=\delta\left(\mathbf{r}-\mathbf{r}^{\prime}\right)
$$

donde $\omega$ es la frecuencia angular y $k=\omega / a_{o}$ es el número de onda acústico. La función de Green en dominio tiempo $G\left(\mathbf{r}, \mathbf{r}^{\prime} ; t\right)$ que permite resolver la ecuación de ondas inhomogénea (4.1) puede obtenerse a partir de la transformada de Fourier inversa de su expresión en dominio frecuencia $G\left(\mathbf{r}, \mathbf{r}^{\prime} ; \omega\right)[79]$

$$
G\left(\mathbf{r}, \mathbf{r}^{\prime} ; t\right)=\frac{1}{2 \pi} \int_{-\infty}^{\infty} G\left(\mathbf{r}, \mathbf{r}^{\prime} ; \omega\right) e^{-i \omega t} d \omega
$$


De modo que para el caso particular de propagación en el espacio libre su forma temporal y frecuencial es

$$
G\left(\mathbf{r}, \mathbf{r}^{\prime} ; \omega\right)=\frac{-e^{-i k\left|\mathbf{r}-\mathbf{r}^{\prime}\right|}}{4 \pi\left|\mathbf{r}-\mathbf{r}^{\prime}\right|} \quad y \quad G\left(\mathbf{r}, \mathbf{r}^{\prime} ; t\right)=\frac{\delta\left(t-\left|\mathbf{r}-\mathbf{r}^{\prime}\right| / a_{o}\right)}{4 \pi\left|\mathbf{r}-\mathbf{r}^{\prime}\right|}
$$

Generalmente, se adoptan las formas asintóticas de las funciones de Green para la propagación en el espacio libre, decayendo como $1 / r$, donde $r \equiv\left|\mathbf{r}-\mathbf{r}^{\prime}\right|$ representa la distancia del punto fuente al punto de observación. Estas formas se refieren al campo acústico lejano y son, según Ribner, más débiles en el campo próximo en comparación con el campo de presión 'pseudosonora' dentro del flujo, el cual depende de los términos de $G(\mathbf{r}, t)$ que decaen como $1 / r^{n}$, que por otra parte son excluidos en la expresión del campo lejano.

Para desarrollar un método eficiente y adecuado del cálculo de los efectos de la refracción del flujo en chorros (figura 4.1), Tam y Auriault [164] proponen la idea de la aplicación de la propiedad de reciprocidad en la función de Green

$$
G\left(x_{o}, x_{s}, \omega\right)=G\left(x_{s}, x_{o}, \omega\right)
$$

la cual manifiesta que la función de Green no cambia cuando los puntos de observación y fuente son intercambiados, convirtiendo así el problema de la refracción en un problema de radiación o scattering. En la misma línea, Ribner [149] comenta que la función de Green referida a un punto fuente estacionario delimita la refracción denominada 'cono de silencio'.

Trabajos anteriores a los de Ribner y Tam, como el realizado por Succi [161], tenían en cuenta efectos del flujo medio sobre la función de Green, aunque no especialmente el de refracción de las ondas sonoras en el cono de silencio. Succi obtuvo la función de Green para la onda plana en un conducto con flujo medio, mediante la introducción de los coeficientes de reflexión en ambos extremos del tubo y el número de Mach, $M$, del flujo medio.

En estudios más actuales, como los presentados por Howe, se obtienen métodos alternativos de la obtención o representación analítica de las funciones de Green. De este modo Howe en un trabajo reciente [80], manifiesta la posibilidad de obtener representaciones analíticas de la función de Green que describan la generación de ondas sonoras por fuentes localizadas en los alrededores del extremo abierto de un cilindro, cuyo extremo se encuentra enrasado con una pared. Estas representaciones analíticas de la función de Green son obtenidas a través de la aplicación del método de Rayleigh para el cálculo aproximado del flujo potencial. 


\subsubsection{Descomposición del campo de velocidades del fluido}

El último esfuerzo teórico que quedaría por mencionar es el de obtener un modelo lineal de las ecuaciones del campo acústico. En general, en la mecánica de fluidos se emplea el vector campo de velocidad de la partícula en lugar de una velocidad volumétrica, escalar, del fluido, lo que nos indica que los modelos aeroacústicos poseen más grados de libertad que los modelos unidimensionales generalmente empleados en los cálculos acústicos. Este vector campo, tridimensional, puede descomponerse en dos vectores campo tridimensionales, bajo condiciones poco restrictivas, con la finalidad de dar solución al problema general de descomposición de un flujo no estacionario en un campo acústico y en un flujo de fondo, hipótesis establecida en todos los capítulos anteriores. En este sentido la propuesta de separación de los campos dada por McGowan [114] y defendida por multitud de autores corresponde a los siguientes campos de velocidad

$$
\mathbf{u}=\mathbf{u}_{s}+\mathbf{u}_{I}
$$

donde $\mathbf{u}_{s}$ representa la componente solenoidal del campo de velocidades y $\mathbf{u}_{I}$ la componente irrotacional de dicho campo. Según McGowan [114] el campo de velocidad en cada punto del espacio se puede escribir como la suma de estos dos tipos de vectores. La parte solenoidal da soporte a movimientos rotacionales del fluido, pero no a compresiones y expansiones, mientras que, la parte irrotacional puede mantener compresiones y expansiones, pero no rotaciones. Razón por la cual, el campo irrotacional constituye una parte necesaria para describir la generación del campo acústico. Estas propiedades pueden enunciarse matemáticamente mediante las siguientes identidades: la divergencia del campo solenoidal es cero y el rotacional del campo irrotacional es cero

$$
\nabla \cdot \mathbf{u}_{s}=0, \quad \nabla \times \mathbf{u}_{I}=0
$$

Esta forma de descomposición del campo también fue de algún modo introducida por Lighthill [104] quien afirmaba que la parte rotacional del campo de velocidad inducida por el campo de vorticidad $\boldsymbol{\Omega}=\nabla \times \mathbf{u}$, debe ser independiente del tiempo en la teoría lineal, ya que este campo verifica $\partial \boldsymbol{\Omega} / \partial t=0$ para que se cumpla la ecuación del momento, (ver ecuación (4.11)), puesto que el rotacional de $\nabla p$ debe anularse. De este modo, según Lighthill, únicamente la parte del campo que es irrotacional representa las fluctuaciones asociadas con la propagación del sonido, no mostrando interacción con ningún campo de flujo estacionario rotacional en la teoría acústica lineal; despreciando los cambios producidos por la convección y asumiendo una vorticidad estacionaria. 
La descomposición de la ecuación (4.42) ha sido muy útil para describir la producción y propagación del sonido, así como la interacción del sonido con otros modos del movimiento del fluido. De cualquier forma, una de las cuestiones más relevantes con respecto a este tema, es si $\mathbf{u}_{I}$ puede atribuirse solamente al campo sonoro, y $\mathbf{u}_{s}$ al flujo de fondo. Ciertamente, en general, la velocidad del flujo de fondo puede descomponerse como suma de componentes irrotacional y solenoidal, y la velocidad del campo acústico puede dividirse de modo similar, como corrobora Fedorchenko [63]. Además, algunos autores como Crow [114] mostraron que las fluctuaciones de presión asociadas a cambios en el campo solenoidal podían propagarse al campo irrotacional como sonido. Resumiendo, puede decirse que desde el punto de vista de la aeroacústica se da cabida a dos tipos de campos de velocidad: uno irrotacional para compresiones y expansiones, el cual mantiene movimientos acústicos, y otro solenoidal, el cual no da soporte a este tipo de movimiento pero proporciona una fuente de sonido, a pesar de que se piensa que este sonido no puede propagarse en este campo (solenoidal) de forma aislada.

Una forma alternativa de descomponer el flujo fue la propuesta por Doak [63], que consiste en suponer que

$$
\rho \mathbf{u}=\mathbf{b}_{t}-\mathbf{b}_{a t}, \quad \nabla \mathbf{b}_{t}=0 \quad \mathbf{b}_{a t}=\nabla \varphi
$$

donde $\mathbf{b}_{t}$ es considerado como una componente 'turbulenta', y $\mathbf{b}_{a t}(\nabla \varphi)$ se asume que será una suma de una parte 'acústica' y una 'térmica', de modo que si el flujo es potencial $(\mathbf{u}=-\nabla \phi)$ la vorticidad es nula, $\nabla \times \mathbf{u}=0$, y según Doak [55] el problema se reduce a un caso más simple.

Sin embargo, estas definiciones conceptuales dan lugar a multitud de cuestiones. En primer lugar si se considera la ecuación de continuidad, se puede observar que las expresiones $\nabla \rho \mathbf{u}$ y $\partial \rho / \partial t$ no son invariantes Galileanas (en contraste con $\nabla \mathbf{u})$. Ciertamente, si $\{\nabla(\rho \mathbf{u})\}_{0}=0$ en el sistema de referencia original (denotado por el subíndice cero), si nos movemos a un nuevo sistema de referencia (denotándolo por el subíndice $m$ ), el cual se mueve con velocidad $\mathcal{U}$ constante relativa al sistema de referencia original se tiene que

$$
\begin{aligned}
\{\mathbf{u}\}_{m} & =\{\mathbf{u}\}_{0}-\mathcal{U}, \quad\{\nabla(\rho \mathbf{u})\}_{m}=\{\mathbf{u}\}_{m} \nabla \rho+\rho \nabla\{\mathbf{u}\}_{m}= \\
& =\{\nabla(\rho \mathbf{u})\}_{0}-\mathcal{U} \nabla \rho=-\mathcal{U} \nabla \rho \neq 0
\end{aligned}
$$

lo que índica que la descomposición anterior (4.44) de $\rho \mathbf{u}$ puede ser implementada solamente en un "único" sistema de referencia. Por otro lado el potencial $\varphi$ así como el término $\Delta \varphi$ deben cambiar dependiendo del sistema de referencia escogido para mantener la validez de la ecuación de continuidad. Consecuentemente, se concluye que esta forma de descomposición dada por Doak, es conceptualmente interesante pero probablemente, un procedimiento poco apropiado de descomposición del flujo en aeroacústica. 


\subsection{Estudios experimentales en aeroacústica}

El análisis de las aproximaciones 'puramente teóricas' en aeroacústica estaría incompleto si no se complementara con una revisión de los principales fenómenos aeroacústicos abordados en la investigación experimental y computacional. El objetivo de este apartado es comentar algunos trabajos experimentales orientados a confirmar la validez de las aproximaciones a la teoría de sonido aerodinámico comentadas en la sección anterior, dejando el estudio computacional para el capítulo siguiente.

En esta sección subrayaremos las restricciones inherentes a la investigación experimental, y se mencionará la necesidad de desarrollar modelos matemáticos más eficientes y algoritmos para la simulación numérica de los fenómenos aerocústicos menos estudiados, particularmente los procesos de generación sonora por flujos altamente inestables.

Los numerosos resultados experimentales existentes en la literatura suelen citarse como confirmación de la validez del modelo básico de Lighthill y el resto de las aproximaciones mencionadas. A partir de diversos trabajos se puede extraer como conclusión fundamental, el hecho de que no hay medidas acústicas en campo libre que puedan revelar de una manera unívoca la distribución de fuentes sonoras en el interior de la región del flujo donde se genera sonido, de modo que las sondas de campo libre no son, presumiblemente, suficientes para el estudio de los mecanismos locales de generación sonora [63]. Por otro lado, las sondas acústicas pueden introducir multitud de perturbaciones en el flujo y cambiar significativamente sus propiedades, hasta el punto de que experimentalmente parece imposible estimar con precisión todas las componentes de una posible fuente sonora justo en la región donde se posee un flujo altamente inestable.

Como referencia a este hecho Dowling y Ffowcs Williams llegan a afirmar, [58] que "no es adecuado escuchar un sonido, o incluso analizar su estructura con las técnicas y equipos más sofisticados, si el objetivo es describir su fuente con certeza".

De este modo, en principio puede afirmarse que la ausencia de datos experimentales fiables, que podrían proporcionar ideas sobre la distribución de fuentes sonoras en un flujo inestable, imposibilita la verificación de cualquier teoría aeroacústica [63]. Razón por la que diferentes modelos teóricos, aunque estos hayan sido invalidados por algunos autores, todavía subsisten. No obstante, se llevan a cabo nuevos experimentos numéricos como intento de justificar el modelo de Lighthill como por ejemplo el estudio de la generación sonora debida a un par de vórtices girantes de Lee y Koo [96] o de un par de vórtices en un chorro axisimétrico, llevado a cabo por Mitchell [117]. En este 
sentido, también han sido planteadas nuevas técnicas de visualización con el fin de adquirir una mayor comprensión de los mecanismos de generación sonora que están presentes en los flujos en estudio. Así, por ejemplo, Lin y Rockwell [107] presentan un sistema de medida con una película de alta densidad de imagen, filmada con la técnica PIV (Particle Image Velocimetry) que permite una visualización detallada del sistema de generación de vórtices, dando la posibilidad de generar representaciones espacio-temporales de la evolución de los patrones de flujo. De esta forma se proporciona una comprensión de los mecanismos físicos que originan las inestabilidades de las interacciones flujoestructura. Esta técnica permite a su vez relacionar los campos de vorticidad y velocidad instantánea con aquellos fenómenos que los inducen, como pueden ser distribuciones de presión superficial. Experimentos similares de visualización fueron llevados a cabo por Nash et al. [126], en este caso se empleó la técnica del LDA (Láser-Doppler Anemometry) la cual permite a su vez la medida de la velocidad del flujo. En el segundo capítulo de esta tesis ya fueron comentadas las ventajas e inconvenientes que presentaban estás técnicas para el caso que nos ocupa.

A pesar de todos los avances realizados en el estudio experimental, Fedorchenko [63] recuerda, que aunque se encuentre un acuerdo satisfactorio entre un experimento y la predicción de ruido realizada mediante el uso de una cierta teoría aeroacústica, esto no puede aceptarse inmediatamente como un argumento decisivo de que el modelo teórico es la mejor aproximación al fenómeno. Se debe tener en cuenta, además, que tal acuerdo puede ser solamente parcial (típicamente, en la comparación de la aproximación de la intensidad sonora en el campo lejano), ya que no se posee el apropiado equipo para realizar todas las comparaciones cuantitativas, mucho menos en el caso del campo próximo. Por otro lado, la expresión diferencial definida como fuente sonora $(q)$ en la ecuación de ondas puede escribirse formalmente como suma de diferentes términos, cada uno de los cuales parece responsable de los diversos efectos físicos que contribuyen a la emisión sonora. Y es posible que un cierto término de esta suma se manifieste como la componente dominante de la fuente sonora real, aunque no haya conexión con el modelo sugerido. Esto pone de relevancia el hecho de que se debe ser cauteloso a la hora de analizar los resultados experimentales, ya que tal y como considera Fedorchenko [63] 'la teoría correcta dará resultados correctos, pero también puede producirlos la teoría errónea'.

Posiblemente, en el futuro, nuevos dispositivos experimentales puedan dar los valores de todas las variables de un flujo altamente inestable, en cualquier instante de tiempo así como en cualquier punto, sin introducir ninguna perturbación. La clave del problema consistirá en separar en cada punto de 
la región del flujo las perturbaciones acústicas, generalmente de muy pequeña amplitud, del flujo de fondo altamente inestable. Sin embargo, este enfoque posee el inconveniente de que cualquier experimento serio no puede hacerse sin una teoría aerodinámica consistente y universalmente aceptada, que proporcione definiciones generales de un flujo de fondo no estacionario, así como también de las ondas sonoras y fuentes sonoras, teoría que hoy por hoy no existe si consideramos las críticas realizadas a la teoría de Lighthill.

A nivel experimental cabe citar que el gran triunfo de la analogía de Lighthill lo constituye su estudio dimensional (sección 4.3.1.5), que permite obtener 'acuerdos completos' entre el nivel de emisión sonora predicho teóricamente o computacionalmente y los valores experimentales. En este sentido, autores como Goldstein y Müller [63] han criticado, aunque débilmente, la disparidad entre los resultados teóricos obtenidos empleando la analogía de Lighthill y algunos datos experimentales, en los que aparecían grandes diferencias respecto a las potencias del número de Mach característico del flujo al analizar flujos subsónicos. Sin embargo, datos experimentales diferentes confirmaban estas leyes de escalado. En este sentido también Lilley [106] en su estudio del sonido radiado por un chorro de aire caliente a bajos números de Mach, manifiesta que este chorro genera una potencia acústica proporcional a $M^{6}$, en vez de la proporcionalidad $M^{8}$ que encontraba Lighthill.

Nelson y Morfey [127] llevaron a cabo un análisis más profundo de estas leyes de escalado. Estos autores realizaron medidas del sonido aerodinámico generado por un alerón inmerso en un conducto por el cual circulaba aire, y que fue modelado a su vez como una distribución de dipolos equivalentes. Consideraron que la potencia sonora radiada estaba relacionada con la fuerza de arrastre total fluctuante que actuaba sobre el alerón, y obtuvieron que para frecuencias por debajo de la frecuencia de corte del primer modo transversal del conducto, la presencia de un conducto infinito modificaba drásticamente la radiación y el dipolo del arrastre se comportaba como un monopolo en campo libre, con la consecuente dependencia de la potencia sonora radiada con la velocidad como $U^{4}$. En cambio, para frecuencias superiores a la frecuencia de corte mencionada, la contribución de todos los modos de propagación puede sumarse para obtener una expresión aproximada de la potencia sonora radiada. Cuando el número de modos propagados aumenta, esta expresión se aproxima a la potencia radiada por un dipolo en campo libre, con la resultante dependencia en $U^{6}$. Estos resultados ponen de manifiesto que en la interpretación y validación de las teorías acústicas por estudios experimentales se deben tener en cuenta multitud de factores, como es por ejemplo, en este caso, el tipo de modos propagados, que indican la dependencia frecuencial del comportamiento de la fuente. 


\subsection{Análisis experimental del ruido de flujo genera- do por geometrías simples}

De acuerdo con los resultados de las secciones anteriores y las interpretaciones del comportamiento de la fuente que ofrecen las leyes de escalado, consideraremos ahora los mecanismos de generación de ruido de flujo asociados tanto a la cánula de escape como a un silenciador, enfrentándonos a la obtención y análisis de estas leyes de escalado para la misma geometría simple analizada en la sección anterior (cámara de expansión reversa). Nuestra pretensión es la de extraer y presentar toda la potencia de la técnica de intensimetría en conductos como técnica experimental para analizar y localizar las fuentes del ruido de flujo generado por los diferentes elementos del sistema de escape, estableciendo así una metodología de análisis experimental con resultados de interés para la industria.

Este estudio de las leyes de escalado se enfoca desde dos puntos de vista:

- Separando las leyes de escalado obtenidas para el ruido de flujo total radiado (que se obtiene a través de la integración de las medidas de intensidad realizadas en la cámara semianecoica para todo el rango de frecuencias considerado), y el ruido de flujo transmitido en el interior del conducto de salida de este elemento (que ahora se obtiene integrando los resultados de la intensidad transmitida estimada mediante la técnica de intensimetría en conductos). Identificando así no sólo qué fenómenos de generación sonora contribuyen al ruido global radiado, sino el diferente peso de estas componentes asociadas a las diferentes fuentes que las generan, internas o externas.

- Obtención de leyes de escalado, en función de las características del flujo, velocidad media o número de Mach, que permitan analizar el comportamiento monopolar, dipolar o cuadripolar de la fuente; y en función de los parámetros geométricos que definen el sistema, lo que sirve como guía para identificar qué mecanismos de generación sonora son responsables de las fuentes dominantes en la emisión global, y la importancia relativa entre ellos. Esto permitirá establecer criterios de diseño para poder reducir alguna contribución determinada al ruido de escape global radiado ya que, como se ha indicado en la sección 4.3.1.5, estas leyes de escalado pueden indicar cual es la principal contribución al ruido emitido en casos realistas en los que varios mecanismos de generación aparecen juntos. 
En primer lugar, comentaremos los resultados obtenidos para la intensidad sonora global radiada obtenida a través de las medidas realizadas en la cámara semianecoica, a $1 \mathrm{~m}$ de la boca de salida y a $60^{\circ}$ del eje del conducto de salida, asegurando así que estamos en condiciones de campo libre y que la posición de medida de los micrófonos no perturba el flujo saliente al encontrarse suficientemente alejados del chorro de descarga. Para representar estos resultados de una forma adimensional adecuada, se utiliza una extensión de los argumentos discutidos en la sección 4.3.1.5, en cuanto a la potencia acústica radiada. El principal punto considerado es que la medida de intensidad a una distancia $r$ de la fuente supone que toda la energía disponible en la descarga puede transformarse en energía acústica y es radiada de forma isótropa. En este sentido la energía medida en un punto localizado a una distancia $r$ de la boca de la cánula de escape, $I$, se asume que tendrá la forma [52]

$$
I=\rho_{o} U^{3}\left(d_{o} / r\right)^{2} \psi
$$

donde $\psi$ es un parámetro adimensional que se identificará a través de las medidas y que puede interpretarse como una eficiencia generalizada de conversión de la energía del flujo en energía acústica, factor que será función de diferentes parámetros dependiendo de la configuración considerada. Su representación resulta conveniente, ya que como puede notarse, la intensidad de referencia (sin reparar en el mecanismo dominante de generación) se refiere a las condiciones en la boca suponiendo que la longitud de escala característica de las estructuras de turbulencia en dicha sección es su diámetro $\left(d_{o}\right)$, pudiéndose obtener información acerca de la predominancia de las fuentes internas y externas. Esto último, sin embargo, se estudiará en mayor profundidad al analizar los resultados derivados de la intensimetría en conductos, ya que la aplicación de estas leyes de escalado a la intensidad radiada indicará únicamente la naturaleza que posee la fuente resultante a la salida de la cánula, pero no permitirá la identificación de la naturaleza de las fuentes aguas arriba de tal sección de salida [52].

Con el fin de tener una visión general sobre las condiciones del flujo en los experimentos realizados, en la tabla contigua se presentan las combinaciones de velocidades y diámetros considerados, derivando así los números de Mach, $M$, y de Reynolds, Re, característicos en cada caso.

\begin{tabular}{|c|c|c|c|}
\hline$d_{o}(\mathrm{~mm})$ & $U(\mathrm{~m} / \mathrm{s})$ & $R e \times 10^{5}$ & $M$ \\
\hline 51 & $34 \rightarrow 80$ & $1.2 \rightarrow 2.7$ & $0.1 \rightarrow 0.24$ \\
\hline 42 & $50 \rightarrow 117$ & $1.4 \rightarrow 3.3$ & $0.14 \rightarrow 0.34$ \\
\hline 30 & $34 \rightarrow 117$ & $0.7 \rightarrow 2.3$ & $0.1 \rightarrow 0.34$ \\
\hline
\end{tabular}


De donde se extrae que el flujo presente en el conducto puede considerarse como un flujo incompresible $(M<0.3)$ y con una turbulencia totalmente desarrollada (variación extremadamente irregular de la velocidad con el tiempo en cada punto), ya que posee números de Reynolds suficientemente grandes en comparación con un número de Reynolds crítico, $R_{c r}$, (límite entre el régimen laminar y turbulento del flujo en una tubería) [95]. Ya que, a pesar de que este $R_{c r}$ no sea una constante universal sino que depende de numerosos factores como el tipo e intensidad de la perturbación realizada al sistema, podemos asegurar que en los diversos sistemas ensayados, aunque las perturbaciones del sistema sean de pequeña intensidad, se alcanzan números de Reynolds suficientes $^{2}$ para romper el flujo laminar metaestable y por tanto tener una turbulencia totalmente desarrollada [95].

Para cubrir un rango suficientemente amplio de las dimensiones de la geometría analizada representada en la figura 3.13 (cámara de expansión reversa), se evalúan 12 geometrías de este tipo variando los parámetros que la definen (si se considera fija la sección de la cámara): diámetro de la cánula de salida $d_{o}$, diámetro de la cánula de entrada $d_{i}$, longitud de la cámara $L_{c}$ y distancia entre las cánulas $d_{c}$. Estos parámetros se modifican dentro de los siguientes márgenes:

\begin{tabular}{|c|c|c|c|}
\hline$d_{o}(\mathrm{~mm})$ & $d_{i}(\mathrm{~mm})$ & $d_{c}(\mathrm{~mm})$ & $L_{c}(\mathrm{~mm})$ \\
\hline $51,42,30$ & $51,47,42,30$ & 73,146 & $25,50,100,150$ \\
\hline
\end{tabular}

En este tipo de cámaras al menos tres fuentes de generación de turbulencia deben estar presentes: (i) el chorro de salida, (ii) el cambio brusco de sección (expansión) alrededor de la salida de la cánula de entrada y (iii) el cambio brusco de sección (contracción) alrededor de la entrada de la cánula de salida. Sin embargo, el comportamiento y la naturaleza de estas fuentes no tiene por qué ser el mismo, razón por la que parece conveniente realizar una representación de la energía cinética turbulenta, k, generada en el interior de estas geometrías mediante una simulación numérica realizada a través de los modelos CFD implementados en el código comercial de Fluent. El hecho de escoger la energía cinética turbulenta, $\mathrm{k}$, como guia de análisis de las fuentes sonoras se debe a los resultados obtenidos por Freund [67], quien afirma que los máximos de la componente radiada de la fuente coinciden con los máximos de energía cinética turbulenta.

\footnotetext{
${ }^{2} \mathrm{Si}$ bien es cierto que el flujo estacionario en una tubería es, efectivamente, estable respecto a pequeñas perturbaciones y puede, en principio, tener lugar para valores de $R e$ considerablemente superiores a $R_{c r}$.
} 
En los cálculos computacionales realizados se considera un flujo estacionario incompresible, de acuerdo con los rangos del número de Mach considerados, y la turbulencia se modela mediante un modelo $\mathrm{k}-\varepsilon$ convencional. El resultado de esta simulación para dos geometrías diferentes se presenta en la figura 4.5, con la finalidad de poder tener una visión más general de los fenómenos de generación sonora existentes en dichas geometrías. De ella se deduce que efectivamente en el interior del silenciador existen las dos fuentes mencionadas pero estas son de naturaleza aparentemente distinta, observándose a la salida de la cánula de entrada un fenómeno de separación del flujo [160], esto es una separación entre el fluido turbulento y el resto de fluido, (ver figura 4.5(b)), pudiendo contener la turbulencia en esta región tanto ondas planas como modos acústicos de orden superior, y un desarrollo de un máximo de energía cinética en torno a uno de los vértices de la sección de entrada de la cánula de salida (figura 4.5(a)), asociado posiblemente con el desarrollo y enrollamiento de hojas vorticales que se extienden a la salida de la expansión [50].

Por otro lado, de estas figuras puede extraerse la existencia de otra posible fuente, aquella que está relacionada con la interacción del flujo incidente con la pared posterior de la cámara (figura 4.5(a)). En cualquier caso, se debe añadir que la naturaleza, comportamiento e intensidad de las fuentes que se presentan en el interior del silenciador difieren en función de la longitud de la cámara y distancias entre las cánulas de entrada y salida, por lo que éstas representan una guía útil de los factores geométricos e interacciones que deben introducirse en las leyes de escalado.

De acuerdo con estas observaciones, la intensidad sonora medida podría ser función únicamente de $d_{o}, d_{i}, L_{c} \mathrm{y} d_{c} \mathrm{y}$ por supuesto del número de Mach, $M$, de acuerdo con los resultados de la sección 4.3.1.5. En relación a la influencia del diámetro de salida, dimensión característica de la fuente de sonido asociada a la descarga del chorro estacionario, su influencia es extremadamente relevante como se puede deducir de la figura 4.6 donde se representan los espectros medidos para una cámara de expansión reversa de la misma longitud y para el mismo número de Mach del flujo, variando el diámetro de la cánula de salida en función de la frecuencia adimensionalizada con la frecuencia característica de la cánula dada por la ecuación [50]

$$
f_{c}=2 i c\left(1-M^{2}\right) / L_{e}
$$

siendo $i=1,2,3 \ldots$ el valor del múltiplo considerado, $c$ la velocidad del sonido y $L_{e}=L_{t}+0.525 d_{o}$ [52] la longitud efectiva de dicha cánula, donde $L_{t}$ representa la longitud física real de ésta. En esta figura (gráfico 4.6) se observa una disminución diferente de los espectros en función del valor del diámetro 


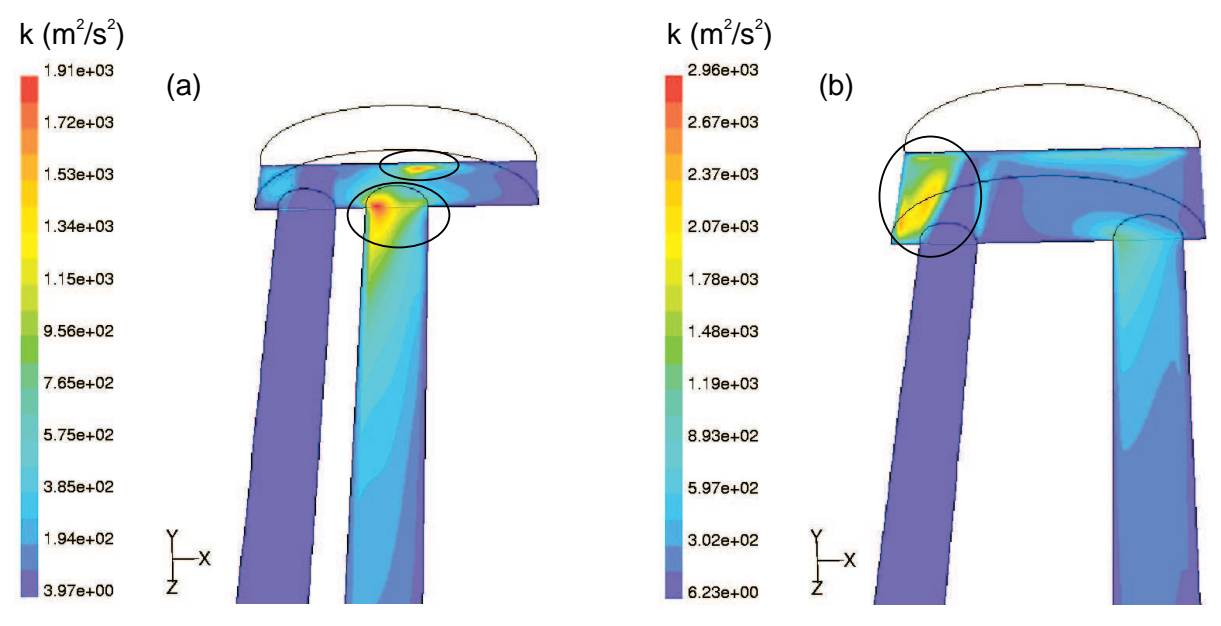

Figura 4.5: Energía cinética turbulenta $k$, simulada para una misma velocidad del flujo medio $(U=100 \mathrm{~m} / \mathrm{s})$ dos geometrías de la cámara de expansión reversa diferentes ( $d_{o}=51 \mathrm{~mm}$ en ambas): (a) $d_{i}=47 \mathrm{~mm}, d_{c}=73 \mathrm{~mm}, L_{c}=50 \mathrm{~mm}$ y (b) $d_{i}=42 \mathrm{~mm}$, $d_{c}=146 \mathrm{~mm}, L_{c}=100 \mathrm{~mm}$.

de la cánula de salida, $d_{o}$, hecho que es corroborado si se representa los valores de intensidad global obtenidos para las diferentes geometrías ensayadas, agrupadas por el diámetro de salida, frente al sonido generado por la descarga de un flujo estacionario en una cánula recta del mismo diámetro [52], como se muestra en la figura 4.7.

El efecto tan dispar que se observa en las gráficas de la figura 4.7 pone de manifiesto la relevancia del diámetro de salida, y justifica la realización de un análisis independiente de los grupos de geometrías que poseen el mismo diámetro de su cánula de salida, para la obtención de las leyes de escalado. Además, pueden adelantarse diferentes consideracionesen relación a estos estudios, ya que el comportamiento relativo que presentan los diferentes grupos frente a la descarga de un chorro libre (ver figura 4.7) permite aventurar que las geometrías de diámetro de salida mayor $d_{o}=51 \mathrm{~mm}(4.7(\mathrm{a}))$ constituyen geometrías generadoras de ruido de intensidad apreciable y por tanto poseerá contribuciones relevantes de todos sus parámetros geométricos; por el contrario, las geometrías de diámetro de salida inferior $d_{o}=30 \mathrm{~mm}(4.7(\mathrm{c}))$ son en parte de su rango geometrías reductoras de ruido frente al emitido por un chorro libre y por tanto es posible que no existan importantes interacciones entre sus factores geométricos, que actúan como amplificadores de ruido. 


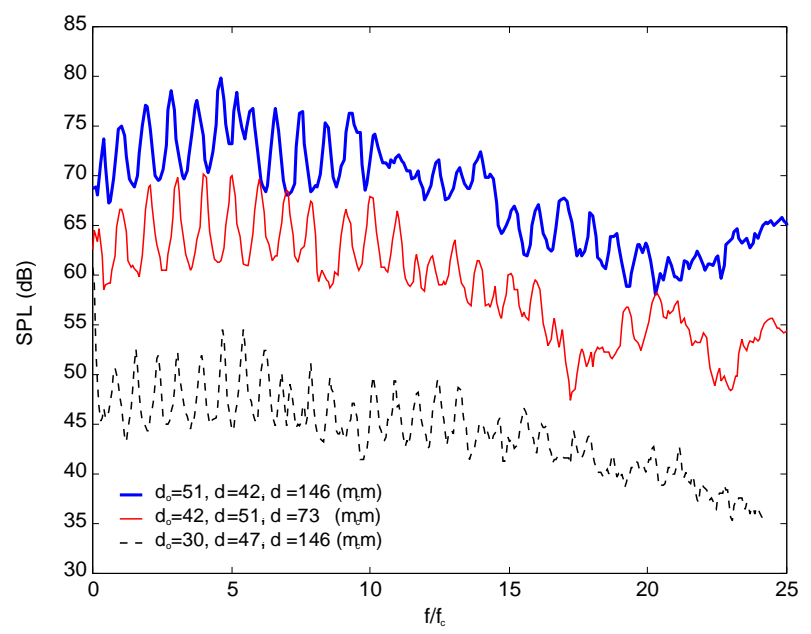

Figura 4.6: Espectros obtenidos para una cámara de expansión reversa de $100 \mathrm{~mm}$ de longitud, para un flujo de número de Mach 0.24.
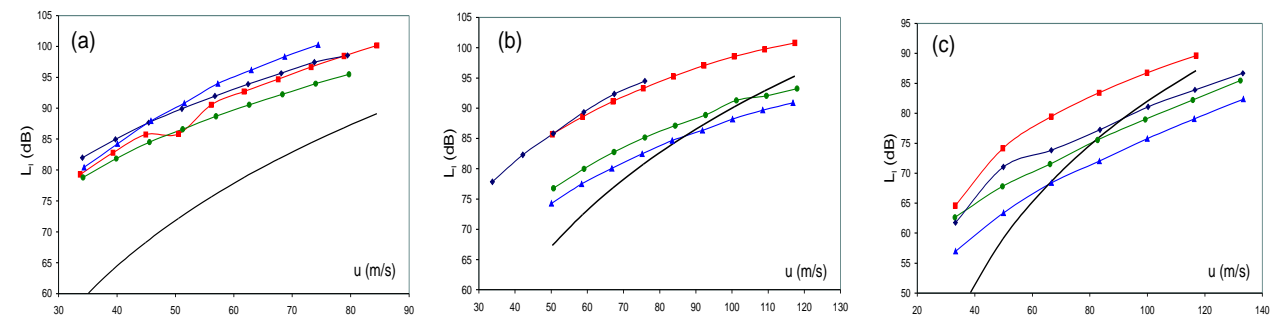

Figura 4.7: Representación del nivel de intensidad global radiado por los diferentes esquemas ( $L_{c}=25, L_{c}=50$, $\bullet L_{c}=100$, $\boldsymbol{\Delta} L_{c}=150 \mathrm{~mm}$,) para los tres diámetros de salida considerados: (a) $d_{o}=51 \mathrm{~mm}$, (b) $d_{o}=42 \mathrm{~mm}$, (c) $d_{o}=30 \mathrm{~mm}$, frente al ruido generado por la descarga de un chorro estacionario (linea continua) en una cánula del mismo diámetro.

Con respecto a los valores de ruido global radiado por cada grupo de geometrías de un mismo $d_{o}$, las variables que definirán su emisión serán el resto de parámetros geométricos $\left(d_{i}, L_{c}\right.$ y $\left.d_{c}\right)$ y la velocidad media del flujo considerada a través del número de Mach, $M$. El factor $\psi$ de la ecuación (4.45) será función de estos parámetros, los cuales deben introducirse como factores adimensionales atendiendo a la definición de intensidad sonora en dB, 
adoptando las leyes de escalado buscadas la siguiente forma básica:

$$
L_{I}-L_{I_{r e f}}=10 \cdot \log \psi
$$

siendo $L_{I_{r e f}}=10 \cdot \log \left(\rho_{o} U^{3}\left(d_{o} / r\right)^{2} / I_{0}\right)$, con $I_{0}=10^{-12} \mathrm{Wm}^{-2}$, y asumiendo la siguiente expresión de $\psi$

$$
\psi=C t e \cdot M^{a} \cdot\left(d_{i} / d_{o}\right)^{b} \cdot\left(L_{c} / d_{i}\right)^{c} \cdot\left(d_{c} / d_{i}\right)^{d} \cdot \Psi
$$

donde el término $\Psi$ contiene los posibles términos de interacción entre estos factores geométricos, ya que como se extrae de la simulación numérica efectuada y como confirman algunos autores [143], el estudio paramétrico debe incluir la influencia de la combinación de diferentes parámetros. En esta geometría en particular, se observa que la forma de este término de interacción, $\Psi$, para el conjunto de geometrías de diámetro de salida $d_{o}=51 \mathrm{~mm}$, responde a la siguiente expresión

$$
\Psi=M^{\left[e \cdot\left(\frac{d_{o}}{d_{i}}\right)+f \cdot\left(\frac{L_{c}}{d_{i}}\right)^{2}\right]} \cdot\left(L_{c} / d_{i}\right)^{g M}
$$

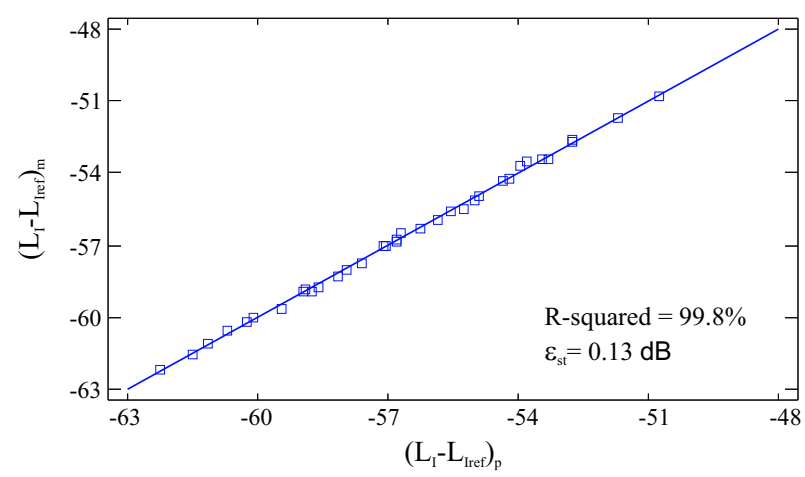

Figura 4.8: Representación de $L_{I}-L_{I_{\text {ref }}}$ obtenidas a través de las intensidades medidas, $\left(L_{I}-L_{I_{\text {ref }}}\right)_{m}$ para las geometrías de $d_{o}=51 \mathrm{~mm}$, en función de la diferencia $\left(L_{I}-L_{I_{r e f}}\right)_{p}$ predicha mediante la ecuación propuesta.

Mostrándose un colapso (reproducción de los valores de ruido global medido) satisfactorio para las cuatro geometrías medidas con este diámetro de salida para los siguientes coeficientes $a=8.3, b=-17.7, c=-2.0, d=-0.23$, $e=-6.5, f=0.25$ y $g=-2.6$, tal y como se recoge en la figura 4.8. Estos resultados muestran que la dependencia con el número de Mach no es sólo un factor constante que informa a primera vista del comportamiento monopolar, 
dipolar o cuadripolar de esta fuente de emisión sino que existe una dependencia de la contribución de los diferentes factores geométricos según la velocidad del flujo asociada al valor de $M$. Hecho que parece evidente si analizamos en la figura 4.9 la diferente intensidad y dependencia con la velocidad media del flujo que presenta la fuente situada a la entrada del tubo de salida, asociada a $d_{o}$, y la localizada cerca de la pared posterior de la cámara, asociada a $L_{c}$.
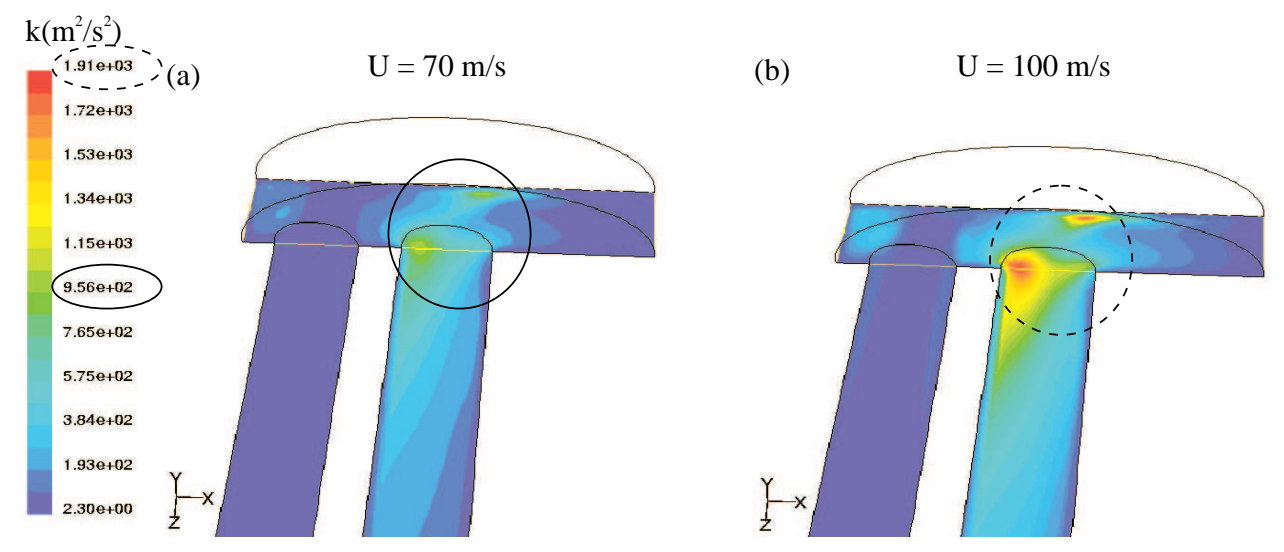

Figura 4.9: Energía cinética turbulenta $k$, simulada para una misma geometrías de la cámara de expansión reversa para dos velocidades del flujo medio: (a) $U=70 \mathrm{~m} / \mathrm{s}$ y (b) $U=100 \mathrm{~m} / \mathrm{s}$.

Un estudio similar se lleva a cabo para las geometrías de diámetro de salida $d_{o}=42 \mathrm{~mm}$, observándose que la expresión del factor $\Psi$ reduce el número de interacciones con la longitud de la cámara y el número de Mach quedando reducido a la siguiente ecuación

$$
\Psi=M^{e \cdot\left(\frac{d_{o}}{d_{i}}\right)} \cdot\left(L_{c} / d_{i}\right)^{g M}
$$

Efecto esperado, ya que el comportamiento del ruido de flujo generado por estas geometrías comienza a aproximarse al de la descarga de un chorro libre como se vio en la figura 4.7(b).

Nuevamente se observa un colapso satisfactorio, mostrado en la figura 4.10 , salvo que ahora se han modificado los valores de los exponentes correspondientes a los diferentes factores, siendo $a=0.57, b=-7.3, c=-1.02$, $d=-0.93, e=0.78$, y $g=1.2$. De la comparación de estos valores con los obtenidos para el diámetro superior se extrae que estos disminuyen en general de valor salvo para la también disminución en valor absoluto pero cambio de 


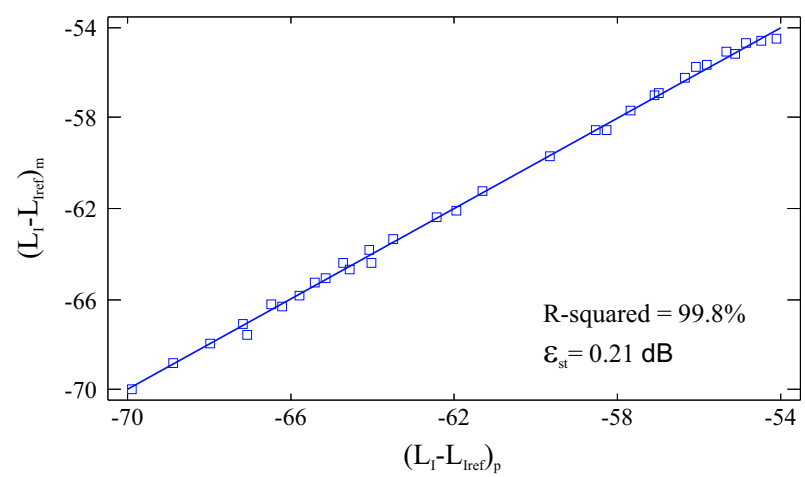

Figura 4.10: Representación de $L_{I}-L_{I_{\text {ref }}}$ obtenidas a través de las intensidades medidas, $\left(L_{I}-L_{I_{\text {ref }}}\right)_{m}$ para las geometrías de $d_{o}=42 \mathrm{~mm}$, en función de la diferencia $\left(L_{I}-L_{I_{\text {ref }}}\right)_{p}$ predicha mediante la ecuación propuesta.

signo de los términos de la interacción, lo cuál corrobora el hecho de que es una fuente de menor intensidad (ver figura 4.7) respecto a las geometrías de $d_{o}=51 \mathrm{~mm}$.

Finalmente, queda por analizar la situación al considerar las geometrías de diámetro inferior, $d_{o}=30 \mathrm{~mm}$, correlación que no muestra ningún término de interacción de los anteriormente mencionados, es decir el parámetro $\Psi=$ 1 , quedando únicamente la contribución de los factores simples $\psi$, situación esperable tras los comentarios realizados sobre la gráfica 4.7(c) ya que estas geometrías, en general, no se muestran como amplificadoras del sonido frente al producido por la descarga de un chorro libre.

Los valores de los exponentes correspondientes a los diferentes factores adoptan ahora los siguientes valores $a=1.1, b=-1.2, c=-0.96$ y $d=0.51$, y reproducen un colapso excelente entre los valores de ruido global medidos y predichos, contenidos en la figura 4.11. Estos exponentes de los factores simples muestran al igual que en el caso anterior, una tendencia de disminución frente a los obtenidos para esquemas de diámetros superiores. Otro aspecto relevante que puede extraerse de esta ley de escalado es la de poseer un exponente con la velocidad cercano a la cuarta potencia (eficiencia de radiación $\psi$ con $M^{1.1}$ ) lo que indicaría un comportamiento monopolar dominante, de acuerdo con los comentarios de la sección 4.3.1.5, resultados que no se pueden aventurar a partir de las leyes de escalado anteriores debido a la complejidad de sus dependencias con el número de Mach. 


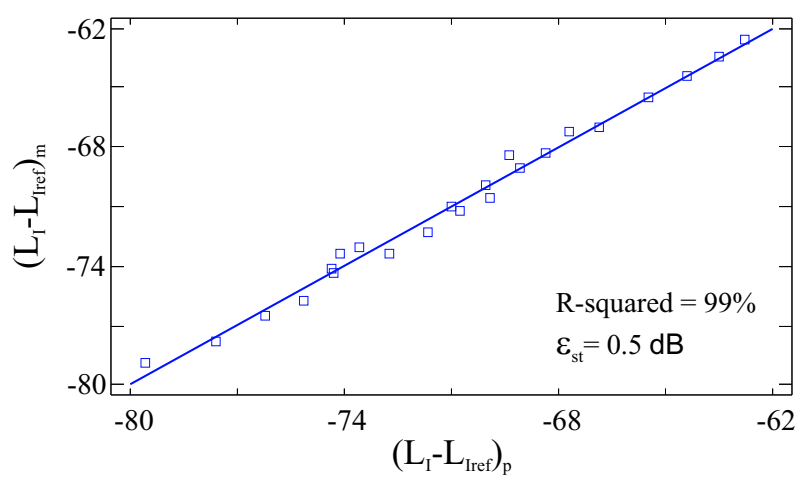

Figura 4.11: Representación de $L_{I}-L_{I_{r e f}}$ obtenidas a través de las intensidades medidas, $\left(L_{I}-L_{I_{r e f}}\right)_{m}$ para las geometrías de $d_{o}=30 \mathrm{~mm}$, en función de la diferencia $\left(L_{I}-L_{I_{\text {ref }}}\right)_{p}$ predicha mediante la ecuación propuesta.

Con el objeto de verificar si estos mismos comportamientos son reproducidos por la fuente interna y presuponiendo que se van a producir ciertos cambios, analizamos los resultados de las mismas ecuaciones de correlación para los valores globales derivados de la intensidad neta estimada a partir de la técnica de intensimetría en conductos. Teniendo en cuenta que el valor global de ruido emitido obtenido de esta forma hace referencia únicamente a los mecanismos de generación interna del silenciador no parece adecuado normalizar, es decir, tomar como intensidad de referencia la potencia de energía disipada por un conjunto de torbellinos presentes en la boca de salida $\rho_{o} U^{3}\left(d_{o}\right)^{2}$, por lo que en este caso adoptaremos como intensidad de referencia un término que de cuenta de la energía neta existente en dicha boca de salida adoptado como

$$
L_{I_{\text {ref }}}=10 \cdot \log \left(\mathrm{K}_{\mathrm{TT}} \rho_{o} U^{3} / I_{0}\right)
$$

siendo $\mathrm{K}_{\mathrm{TT}}$ el coeficiente de perdida de carga de una cámara de expansión reversa.

La justificación de la adopción del término presentado en la ecuación (4.51) podemos encontrarla en el trabajo realizado por Nelson y Morfey [127], donde se analiza el sonido generado por un flujo de aire subsónico en un conducto considerando que existen fuerzas de fluctuación que actúan sobre una lámina emplazada en su interior. Sin embargo, estas fuerzas de fluctuación son imposibles de predecir por lo que se asume que son proporcionales a las fuerzas de resistencia aerodinámica [176], las cuales pueden ser expresadas en función del coeficiente de resistencia de la lámina. Este parámetro a su vez puede obtenerse a partir de la medida de caída de presión estática $(\Delta p)$. 
Finalmente, a través de estas relaciones dadas por Nelson y Morfey [127] y teniendo en cuenta la definición de coeficiente de pérdida de carga [130] $\left(\mathrm{K}_{l}=\right.$ $\Delta p /\left(\rho_{o} U^{2} / 2\right)$, donde el subíndice $l$ hace referencia a que es el producido por la lámina), se obtiene que la fuerza de resistencia que actúa sobre ella puede escribirse como sigue

$$
\mathbf{F}_{r}=\mathrm{K}_{l}\left(\frac{\rho_{o} U^{2}}{2}\right) A_{s}
$$

En el caso considerado, no es una lámina la que produce ésta caída de presión sino los elementos del silenciador, por tanto la fuerza responsable de generar el sonido transmitido en el extremo del conducto será la dada por la ecuación (4.52) sustituyendo en ella el coeficiente de pérdida de carga correspondiente al medido en dicho silenciador $\left(\mathrm{K}_{\mathrm{TT}}\right)$.

Una vez conocida la expresión que adquiere esta fuerza de resistencia aerodinámica, sólo resta obtener la intensidad neta transmitida en el extremo del conducto $\left(I_{r e f}\right)$. Para ello, partiremos de su definición como la potencia transmitida, $W_{t}$, por unidad de área, $A_{s}\left(I_{r e f}=W_{t} / A_{s}\right)$. Esta potencia neta transmitida es fácilmente obtenible teniendo en cuenta que la potencia será la energía neta transmitida por unidad de tiempo $(t)$, siendo esta energía igual al producto de la fuerza responsable de esta emisión, $\mathbf{F}_{r}$, por el desplazamiento característico producido por el movimiento turbulento que genera el sonido $(\ell)$. De acuerdo con todas las relaciones mencionadas y teniendo en cuenta que la velocidad característica del proceso es la velocidad media del flujo $(U=\ell / t)$ la intensidad de referencia adopta la siguiente expresión:

$$
I_{\text {ref }}=\frac{\mathbf{W}_{t}}{A_{s}}=\mathrm{K}_{\mathrm{TT}}\left(\frac{\rho_{o} U^{2}}{2}\right) \cdot\left(\frac{\ell}{t}\right) \propto \mathrm{K}_{\mathrm{TT}} \cdot \rho_{o} U^{2} \cdot U
$$

expresión (4.53) cuyo nivel de intensidad sonora se corresponde con el dado por la ecuación (4.51).

De acuerdo con el término escogido para normalizar la intensidad neta transmitida (ecuación (4.51)), la ley de escalado obtenida para el $d_{o}=51 \mathrm{~mm}$, empleando la ecuación (4.47) donde es sustituido el valor de $L_{I_{\text {ref }}}$ dado por la expresión anterior (4.51) y considerando la forma del factor $\psi$ dado por la ecuación (4.48) y del término $\Psi$ adoptado en este caso (ecuación (4.49)), los exponentes obtenidos son: $a=8.5, b=-10.02, c=-0.38, d=0.6$, $e=-6.4, f=0.23$ y $g=-2.3$. Reproduciéndose un colapso satisfactorio como se observa en la figura 4.12 .

De la comparación de los exponentes de los diferentes factores obtenidos para los valores de intensidad global transmitida y radiada, mostrados más 


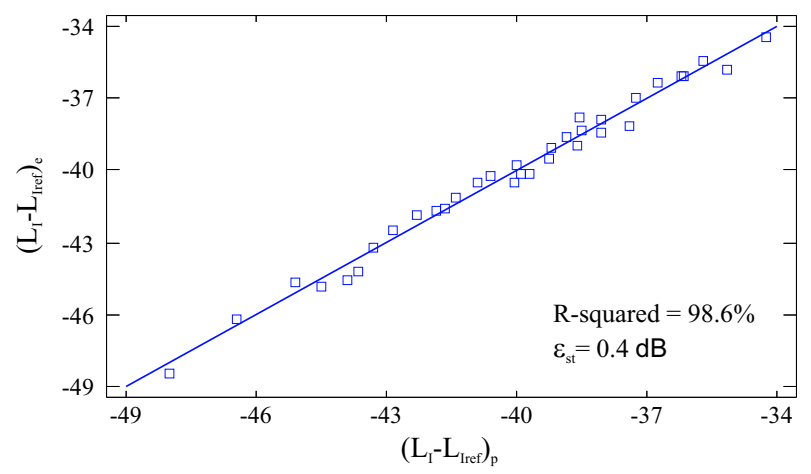

Figura 4.12: Representación de $L_{I}-L_{I_{r e f}}$ obtenidas a través de las intensidades netas estimadas con la intensimetría en conductos, $\left(L_{I}-L_{I_{r e f}}\right)_{e}$ para las geometrías de $d_{o}=51 \mathrm{~mm}$, en función de la diferencia $\left(L_{I}-L_{I_{r e f}}\right)_{p}$ predicha mediante la ecuación propuesta.

claramente en la figura 4.15, se extrae que estos adoptan valores muy similares a excepción de la dependencia con la longitud de la cámara, hecho que puede deberse a que su efecto está contenido en el coeficiente de pérdida de carga introducido en la intensidad de referencia (4.51) y que corresponde a la expresión

$$
\mathrm{K}_{\mathrm{TT}}=1+\left(0.5+k_{\text {corr }}\right)\left(\frac{d_{i}}{d_{o}}\right)^{4} ; \quad k_{\text {corr }}=-0.6 \xi^{3}+1.96 \xi^{2}-2.03 \xi+0.68
$$

donde $\xi=\log \left(L_{c} / d_{o}\right)$. Esta buena reproducción del comportamiento de la predicción de los valores globales de la intensidad radiada a partir de la intensidad transmitida puede justificarse por el hecho de que estas geometrías sean fuentes generadoras de ruido de flujo de gran intensidad en comparación con la descarga de un chorro libre (ver figura 4.7(a)).

El mismo análisis se lleva a cabo para las geometrías de diámetro de salida $d_{o}=42 \mathrm{~mm}$, adoptando ahora la expresión del factor $\Psi$ dada por la ecuación (4.50), reproduciéndose al igual que en los casos anteriores un grado de correlación adecuado como se deduce de la figura 4.13, obtenido para los siguientes exponentes: $a=-0.75, b=-8.9, c=0.42, d=-0.8, e=1.9, \mathrm{y}$ $g=0.7$. Los exponentes obtenidos para estas geometrías reproducen resultados singularmente desviados de los obtenidos para los valores de la intensidad radiada, como se refleja en la tabla 4.15; de todos ellos, quizás el de mayor relevancia lo constituya el cambio no sólo de valor sino también de signo del coeficiente del número de Mach, aunque a este respecto también se debe 
mencionar el incremento del coeficiente de la interacción entre la relación de diámetros y el número de Mach, lo que puede compensar de algún modo la tendencia anterior, efecto que se estudiará más adelante a través de representaciones tridimensionales. Por otro lado, esta posible desviación de tendencias puede atribuirse a que estas geometrías son, en algunos casos, fuentes generadoras de ruido de menor intensidad frente a la descarga del chorro libre (ver figura 4.7(b)), efecto del que no dan cuenta las medidas internas.

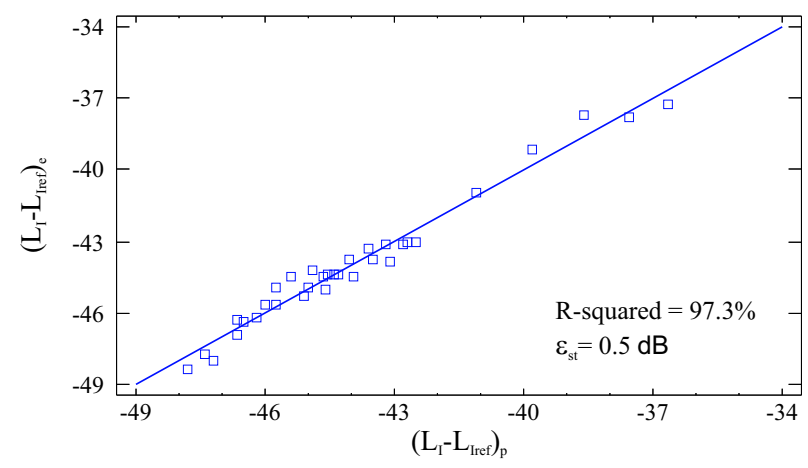

Figura 4.13: Representación de $L_{I}-L_{I_{r e f}}$ obtenidas a través de las intensidades netas estimadas con la intensimetría en conductos, $\left(L_{I}-L_{I_{r e f}}\right)_{e}$ para las geometrías de $d_{o}=42 \mathrm{~mm}$, en función de la diferencia $\left(L_{I}-L_{I_{r e f}}\right)_{p}$ predicha mediante la ecuación propuesta.

Finalmente, sólo cabe mencionar los resultados obtenidos para las geometrías de $d_{o}=30 \mathrm{~mm}$ a través de un estudio análogo, en el que se elimina, como en el caso de la intensidad radiada, la contribución de cualquier posible interacción entre sus factores geométricos y la velocidad del flujo medio. El buen grado de acuerdo predicho mediante esta ley de escalado para los coeficientes $a=0.8, b=-7.9, c=1.4$ y $d=0.18$, se muestra en la figura 4.14. De la comparativa de estos resultados con los obtenidos para la intensidad radiada, contenidos en la figura 4.15, cabe resaltar la mayor contribución del término asociado al diámetro de la cánula de entrada para los valores globales de la intensidad transmitida en el conducto, lo cuál parece lógico si pensamos que este efecto puede quedar enmascarado en la intensidad radiada total por los efectos de la descarga. El resto de términos presenta un comportamiento similar al que se encuentra en la intensidad radiada, reproduciéndose incluso el comportamiento aproximadamente monopolar de la fuente para un exponente del número de Mach de 0.8, algo inferior al obtenido para los valores de intensidad radiada $\left(M^{1.1}\right)$, que se debe al hecho de que el efecto de la descarga amplifica esta contribución. 


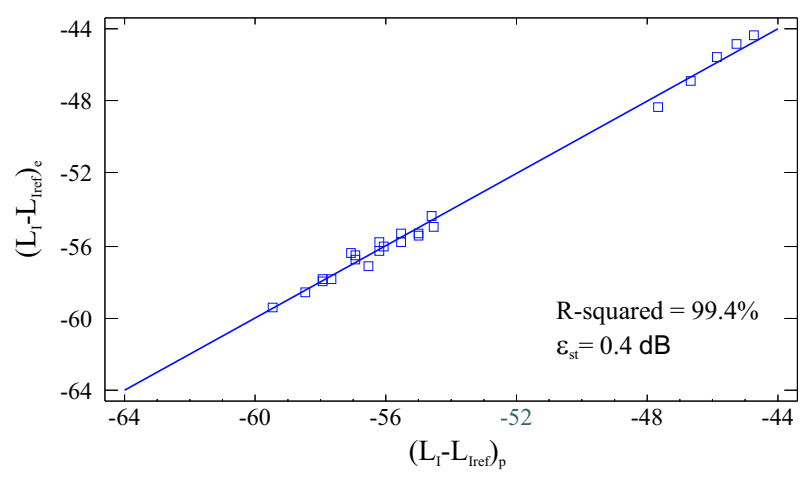

Figura 4.14: Representación de $L_{I}-L_{I_{r e f}}$ obtenidas a través de las intensidades netas estimadas con la intensimetría en conductos, $\left(L_{I}-L_{I_{\text {ref }}}\right)_{e}$ para las geometrías de $d_{o}=30 \mathrm{~mm}$, en función de la diferencia $\left(L_{I}-L_{I_{r e f}}\right)_{p}$ predicha mediante la ecuación propuesta.

De todo el análisis comparativo realizado para las leyes de escalado obtenidas de las medidas de la intensidad radiada (que contiene información tanto de las fuentes internas como de las externas [52]), y de las obtenidas a partir de la intensidad neta transmitida (que da cuenta únicamente de lo que ocurre en el interior del conducto), se deriva como conclusión principal, que el comportamiento de una y otra es más parejo cuando consideramos geometrías generadoras de ruido de flujo de intensidad superior a la de descarga de un chorro libre por un conducto del mismo diámetro de salida. En los otros tipos de geometrías la desviación que presenta el comportamiento de algunos parámetros geométricos puede atribuirse al hecho de que el efecto de la descarga del chorro enmascare algunos de estos efectos de generación interna del ruido de flujo.

Como ya se vio, la complejidad de las leyes de escalado e interacciones que contienen en el caso de geometrías de mayor diámetro de salida, $d_{o}=51$ y $42 \mathrm{~mm}$, no permiten analizar a simple vista el efecto de los parámetros geométricos y velocidad media del flujo sobre el ruido global emitido y por tanto tampoco su comportamiento monopolar, dipolar o cuadripolar. Por ello, y con el fin de extraer y evaluar toda la posible potencia que presenta este método de cara a la mayor comprensión de los mecanismos de generación y comportamientos de las fuentes sonoras por el paso de un flujo estacionario, a continuación se estudian gráficamente las tendencias de evolución que siguen estos factores. 


\begin{tabular}{|c|c|c|c|c|c|c|}
\hline \multicolumn{6}{|c|}{$\left.\left.\Psi=M^{\left[e\left(d_{o} / d_{i}\right)+f\left(L_{c} / d_{i}\right.\right.}\right)^{2}\right]\left(\frac{L_{c}}{d_{i}}\right)^{g M}$} \\
\hline & $\begin{array}{c}\text { Exponentes para la intensidad } \\
\text { radiada (medida) }\end{array}$ & \multicolumn{2}{c|}{$\begin{array}{c}\text { Exponentes para la intensidad } \\
\text { transmitida (estimaciones) }\end{array}$} \\
\hline d $_{\mathrm{o}}$ & $51 \mathrm{~mm}$ & $42 \mathrm{~mm}$ & $30 \mathrm{~mm}$ & $51 \mathrm{~mm}$ & $42 \mathrm{~mm}$ & $30 \mathrm{~mm}$ \\
\hline$a$ & 8.6 & 5.3 & 1.1 & 8.5 & -0.75 & 0.8 \\
\hline $\mathrm{b}$ & -17.7 & -7.3 & -1.2 & -10.02 & -8.9 & -7.9 \\
\hline $\mathrm{c}$ & -2.0 & -1.02 & -0.96 & -0.38 & 0.42 & 1.4 \\
\hline $\mathrm{d}$ & -0.23 & -0.93 & 0.51 & 0.6 & -0.8 & 0.18 \\
\hline $\mathrm{e}$ & -6.5 & 0.78 & & -6.4 & 1.9 & \\
\hline $\mathrm{f}$ & 0.25 & & & 0.23 & & \\
\hline $\mathrm{g}$ & -2.6 & 1.24 & & -2.3 & 0.7 & \\
\hline
\end{tabular}

Figura 4.15: Resumen de los exponentes obtenidos para los factores geometricos en las diferentes leyes de escalado.

\subsubsection{Análisis de las leyes de escalado}

En primer lugar analizaremos cuál es el comportamiento de la intensidad radiada y transmitida por el conducto frente al cambio de la velocidad del flujo, $M$, y de la distancia entre las cánulas $d_{c}$. Ya que, como este último factor no está contenido en ningún término de interacción, permite un análisis directo de las leyes de escalado obtenidas. Estos resultados derivados de las leyes de escalado correspondientes a las geometrías de $d_{o}=51 \mathrm{~mm}$ se muestran en las gráficas 4.16. En ellas se reproduce una tendencia de crecimiento de la intensidad similar con el número de Mach tanto para la intensidad radiada (4.16(a)) como para la transmitida (4.16(c)). Sin embargo el efecto con las distancias de las cánulas no es reproducido por igual en ambos casos. Así, mientras que para la intensidad transmitida (4.16(d)), la intensidad sonora se incrementa al aumentar esta distancia para la intensidad radiada (4.16(b)) se observa un efecto contrario. A este respecto cabe resaltar que las tendencias de evolución no son acusadas en ninguno de los dos casos. Hasta cierto punto 
parece lógico pensar que la medida de intensimetría en conductos proporcione un comportamiento diferente al de la medida exterior donde están contenidos muchos más efectos, por lo que a pesar de la ligera discrepancia observada, estos resultados siguen contribuyendo a la cualificación de la potencia de esta técnica de estimación para reproducir los comportamientos de la geometría analizada.
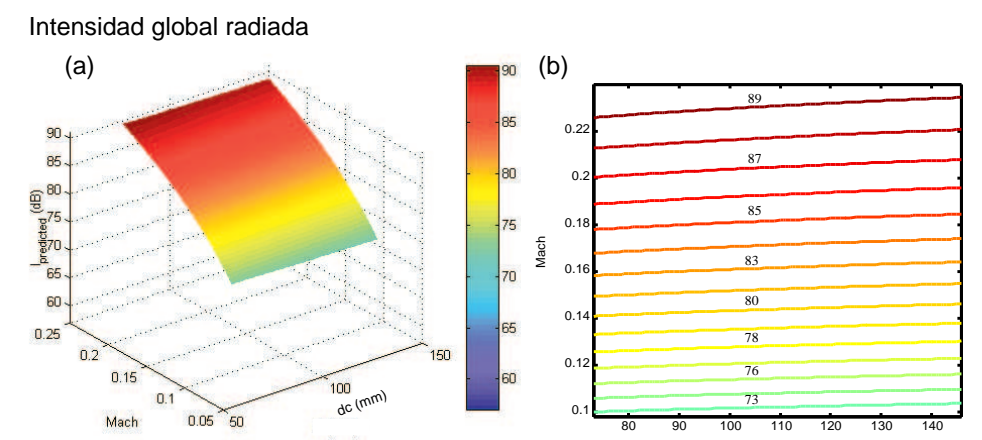

Intensidad global transmitida

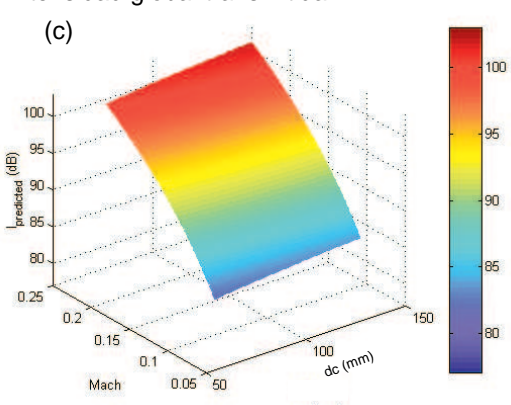

(d)

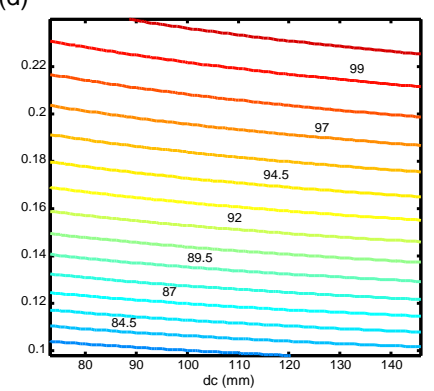

Figura 4.16: Evolución de la intensidad radiada y transmitida, en función del número de Mach y la distancia entre las cánulas $d_{c}$ para las geometrías de $d_{o}=51 \mathrm{~mm}$, en el caso en que el resto de parámetros geométricos permanezcan invariables. Se estudia su aspecto tridimensional (figuras de la izquierda) y su proyección en los mapas de curvas de nivel reproducidas (figuras de la derecha).

Un análisis análogo se lleva a cabo con las leyes de escalado obtenidas para las geometrías de $d_{o}=42 \mathrm{~mm}$, presentado en la figura 4.17 y las de diámetro $d_{o}=30 \mathrm{~mm}$, figura 4.18 , reproduciéndose nuevamente un comportamiento de crecimiento tanto de la intensidad sonora radiada como de la transmitida con el número de Mach. En relación a esta evolución cabe mencionar que las más acusadas son las que presentan las geometrías de $d_{o}=51$ y $42 \mathrm{~mm}$, lo que permite aventurar que si el comportamiento de las geometrías de $d_{o}=30 \mathrm{~mm}$ respondía a una fuente monopolar $(M)$ el de las otras geometrías se asemejará 


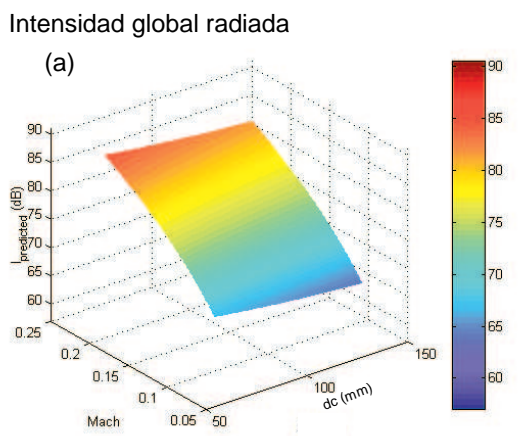

Intensidad global transmitida

(c)

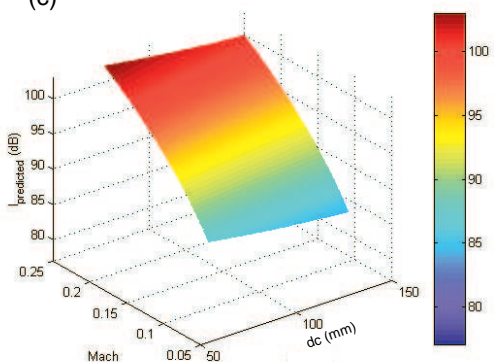

(b)

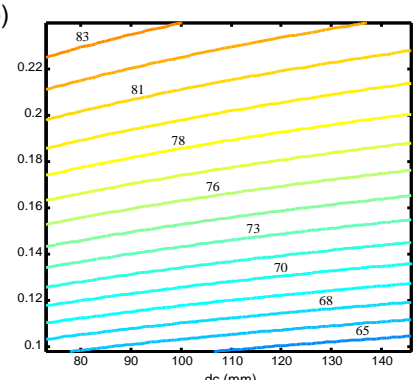

(d)

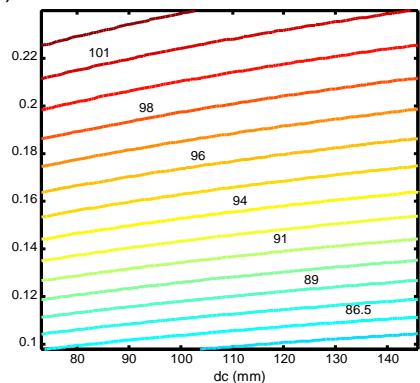

Figura 4.17: Evolución de la intensidad radiada y transmitida, en función del número de Mach y la distancia entre las cánulas $d_{c}$ para las geometrías de $d_{o}=42 \mathrm{~mm}$, en el caso en que el resto de parámetros geométricos permanezcan invariables. Se estudia su aspecto tridimensional (figuras de la izquierda) y su proyección en los mapas de curvas de nivel reproducidas (figuras de la derecha).

al de una fuente entre monopolar y dipolar $\left(M^{3}\right)$, efecto que no era fácilmente predecible debido al grado de complejidad de las leyes de escalado en estos casos.

Con respecto a la tendencia de la intensidad sonora radiada y transmitida con la distancia entre las cánulas puede observarse (figuras 4.17 (b y d) y 4.18 (b y d)) que para ambos casos la intensimetría en conductos reproduce el mismo comportamiento que los obtenidos para las medidas de la intensidad radiada. Sin embargo, la diferencia estriba en que así como las geometrías de $d_{o}=42 \mathrm{~mm}$ (figuras 4.17 (b y d) muestran una tendencia de disminución de la intensidad sonora generada al aumentar la distancia entre las cánulas para un número de Mach fijo, las geometrías de $d_{o}=30 \mathrm{~mm}$ (4.18 (b y d)) reproducen un comportamiento opuesto; sin ser acusada ninguno de las dos tendencias. 


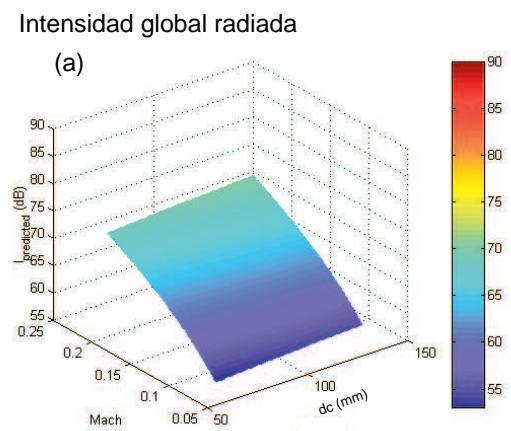

Intensidad global transmitida

(c)

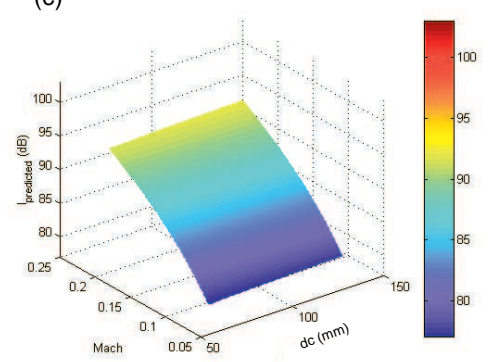

(b)

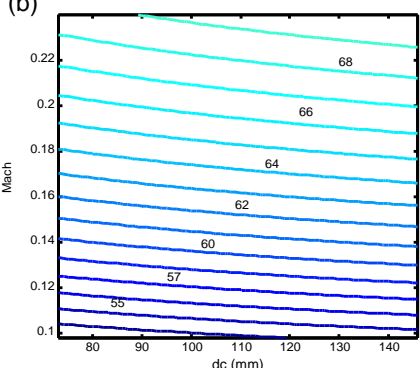

(d)

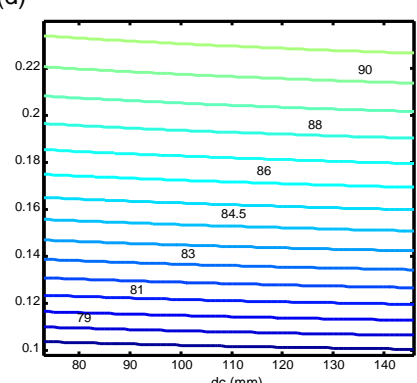

Figura 4.18: Evolución de la intensidad radiada y transmitida, en función del número de Mach y la distancia entre las cánulas $d_{c}$ para las geometrías de $d_{o}=30 \mathrm{~mm}$, en el caso en que el resto de parámetros geométricos permanezcan invariables. Se estudia su aspecto tridimensional (figuras de la izquierda) y su proyección en los mapas de curvas de nivel reproducidas (figuras de la derecha).

Una vez analizados los comportamientos frente a los términos simples de las leyes de escalado obtenidas pasamos a considerar los efectos de las interacciones de la longitud de la cámara, $L_{c}$, y relación de diámetros $\left(d_{o} / d_{i}\right)$, con la velocidad del flujo. En primer lugar, abordaremos el efecto de la longitud de la cámara ya que este término presenta una doble contribución para el caso de las geometrías de $d_{o}=51 \mathrm{~mm}$ (ver el valor del factor $\Psi$ para estas geometrías ecuación (4.49)). Para ello se realizan representaciones similares a las anteriores, contenidas en la figura 4.19, donde se observa un acuerdo excelente en la reproducción de las tendencias de las medidas de intensidad radiada (4.19 (a y b)) por parte de las intensidades estimadas en la cánula, intensidad transmitida (4.19 (c y d)), en relación a una tendencia de disminución de la intensidad sonora al aumentar la longitud de la cámara. 


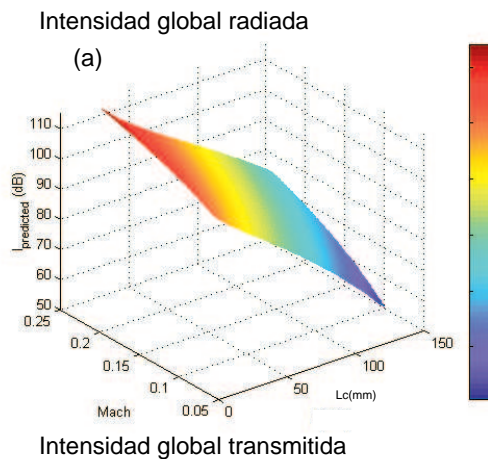

Intensidad global transmitida

(c)

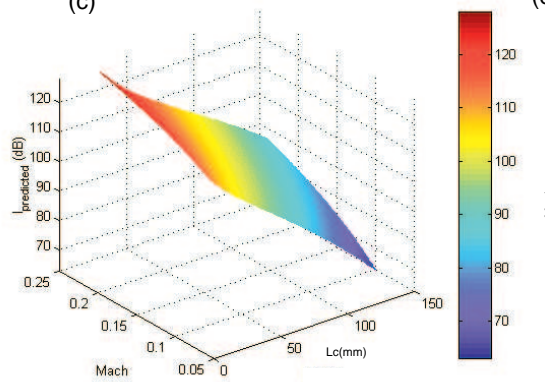

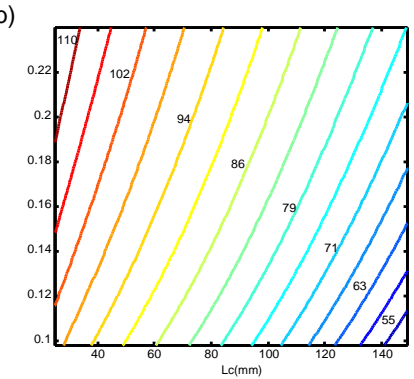

(d)

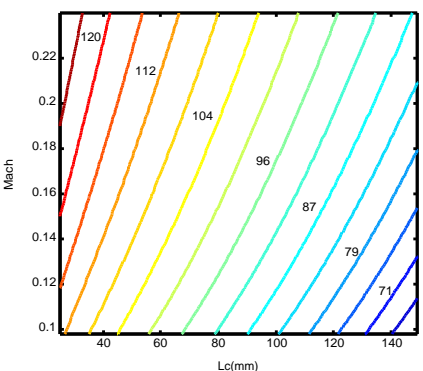

Figura 4.19: Evolución de la intensidad radiada y transmitida, en función del número de Mach y la longitud de la cámara $L_{c}$ para las geometrías de $d_{o}=51 \mathrm{~mm}$, en el caso en que el resto de parámetros geométricos permanezcan invariables. Se estudia su aspecto tridimensional (figuras de la izquierda) y su proyección en los mapas de curvas de nivel reproducidas (figuras de la derecha).

Con respecto a los resultados obtenidos para las geometrías de diámetros inferiores, $d_{o}=42$ y $30 \mathrm{~mm}$, en las gráficas 4.20 y 4.21 se observa la evolución con la $L_{c}$. En este caso, las tendencias de disminución de intensidad radiada al aumentar la longitud de la cámara, $L_{c}$, que muestran las medidas, no son reproducidas por las leyes de escalado obtenidas para la intensidad transmitida en ninguno de los dos casos. De forma que a pesar de que sí que se reproducen comportamientos poco acusados estos muestran ahora un aumento de la intensidad transmitida al aumentar la longitud de la cámara (4.20(d) y 4.21(d)), desviación que será justificada más adelante.

Finalmente, se analiza el efecto de la segunda interacción entre la relación de diámetros, $\left(d_{o} / d_{i}\right)$ y el número de Mach, $M$, reproduciéndose para todos los casos la disminución observada en las medidas de la intensidad sonora radiada al aumentar el diámetro de la cánula de entrada $d_{i}$, por todas 


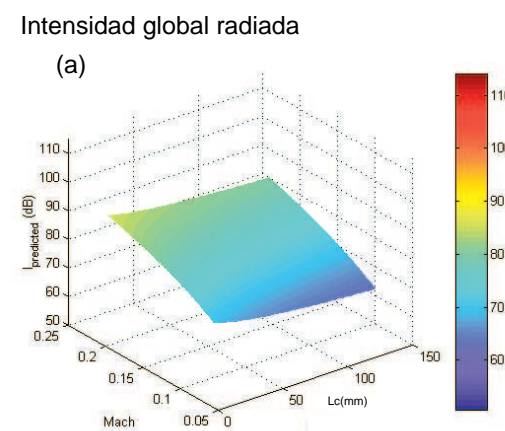

Intensidad global transmitida

(c)

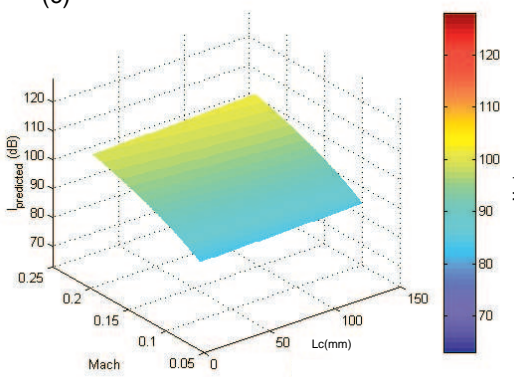

(b)

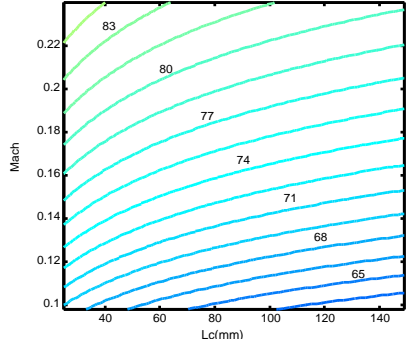

(d)

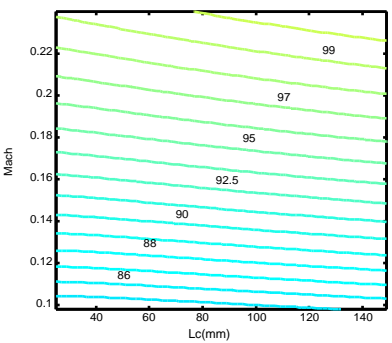

Figura 4.20: Evolución de la intensidad radiada y transmitida, en función del número de Mach y la longitud de la cámara $L_{c}$ para las geometrías de $d_{o}=42 \mathrm{~mm}$, en el caso en que el resto de parámetros geométricos permanezcan invariables. Se estudia su aspecto tridimensional (figuras de la izquierda) y su proyección en los mapas de curvas de nivel reproducidas (figuras de la derecha).

las correlaciones obtenidas de la intensidad transmitida, ver figuras de 4.22 a 4.24. Sin embargo, pueden extraerse varios comentarios de estas figuras; en primer lugar, así como para las geometrías de $d_{o}=51 \mathrm{~mm}$ se observa una reproducción exacta del comportamiento observado con las intensidades medidas (figura 4.22), para las geometrías de $d_{o}$ inferior se reproduce un comportamiento ligeramente más acusado de esta tendencia para las medidas de la intensidad transmitida (figuras 4.23(d) y 4.24(d)) e incluso algo más acusado para el diámetro $d_{o}=30 \mathrm{~mm}$. Efectos lógicos desde el punto de vista de que las medidas internas en el conducto contendrán mayor información sobre los efectos del diámetro de entrada, $d_{i}$, que las medidas externas realizadas, ya que a estas segundas se debe añadir el efecto de la descarga del flujo, efecto especialmente importante para las geometrías de $d_{o}=30 \mathrm{~mm}$ que constituyen fuentes generadoras de ruido de flujo cuya contribución final es del mismo orden que la descarga de un chorro libre (ver figura 4.7(b y c)). 


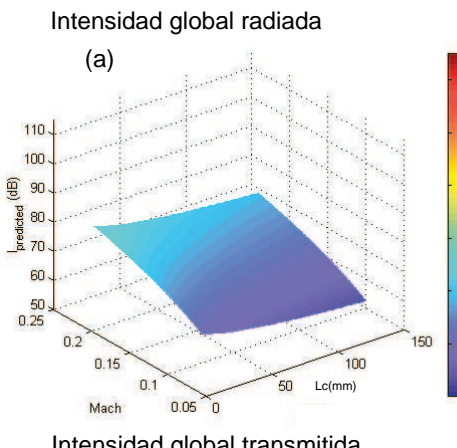

tensidad global transmitida (c)

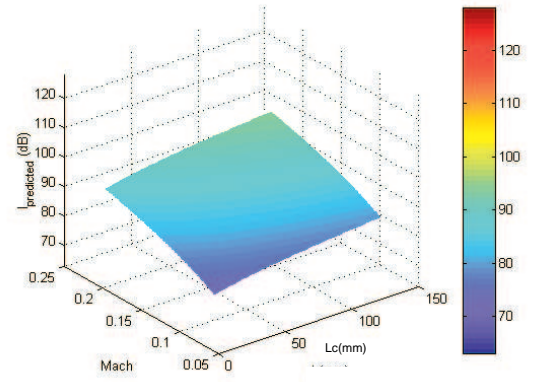

(b)

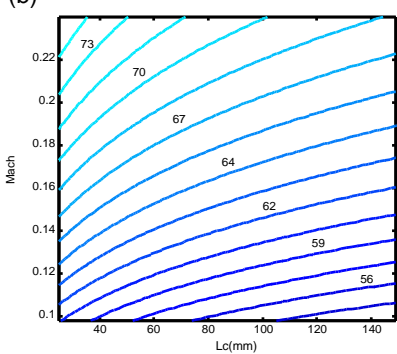

(d)

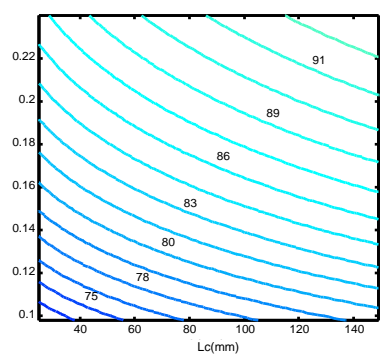

Figura 4.21: Evolución de la intensidad radiada y transmitida, en función del número de Mach y la longitud de la cámara $L_{c}$ para las geometrías de $d_{o}=30 \mathrm{~mm}$, en el caso en que el resto de parámetros geométricos permanezcan invariables. Se estudia su aspecto tridimensional (figuras de la izquierda) y su proyección en los mapas de curvas de nivel reproducidas (figuras de la derecha).

De todos los resultados anteriores se pueden extraer las siguiente conclusiones.

- Las leyes de escalado derivadas para las geometrías de $d_{o}=51 \mathrm{~mm}$ a partir de las medidas de intensimetría en conductos, reproducen los efectos observados en las medidas de intensidad radiada en una cámara semianecoica, lo que garantiza que esta metodología de estudio del ruido de flujo sea idónea para aquellos elementos de escape que presenten una generación sonora de ruido de flujo superior a la de descarga de un chorro libre (ver figura 4.7(a)).

- Para las geometrías de diámetros de salida inferiores, $d_{o}=42$ y $30 \mathrm{~mm}$, se reproducen también tendencias muy aproximadas a las observadas en las medidas externas a excepción de los siguientes comportamientos: 

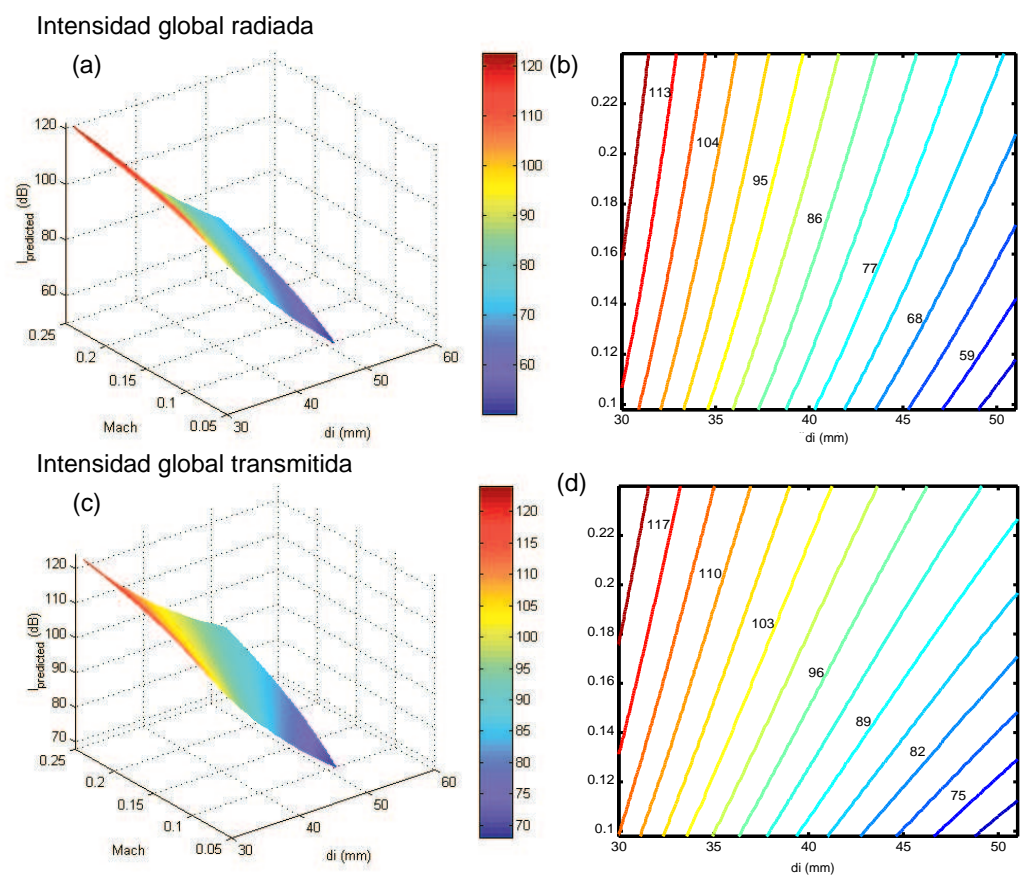

Figura 4.22: Evolución de la intensidad radiada y transmitida, en función del número de Mach y el diámetro de la cánula de entrada $d_{i}$ para las geometrías de $d_{o}=51 \mathrm{~mm}$, en el caso en que el resto de parámetros geométricos permanezcan invariables. Se estudia su aspecto tridimensional (figuras de la izquierda) y su proyección en los mapas de curvas de nivel reproducidas (figuras de la derecha).

1. Las leyes de escalado obtenidas para las intensidades transmitidas muestran una tendencia de evolución más acusada con el $d_{i}$ que las derivadas de las medidas de intensidad realizadas en el exterior (cámara semianecoica), lo que ratifica que el método de intensimetría en conductos proporciona mayor información sobre las fuentes de generación interna que las medidas externas donde sus efectos coexisten con los de la descarga del flujo, pudiendo incluso quedar enmascarados o atenuados por ellos, como en este caso (ver figura 4.7 (b y c)).

2. Las leyes de escalado obtenidas de las medidas internas reproducen una evolución contraria con la longitud de la cámara (aumento de la intensidad al aumentar la longitud de la cámara), hecho que viene justificado para la elección del punto de medida de presión en el interior de la cánula por dos motivos: por un lado, como 


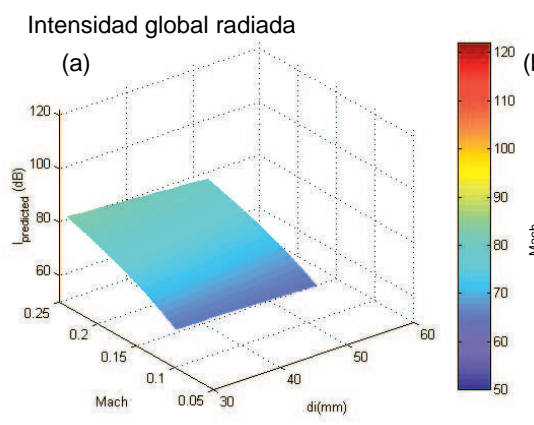

Intensidad global transmitida

(c)

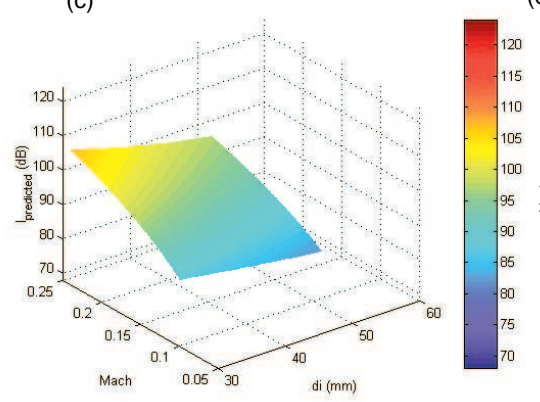

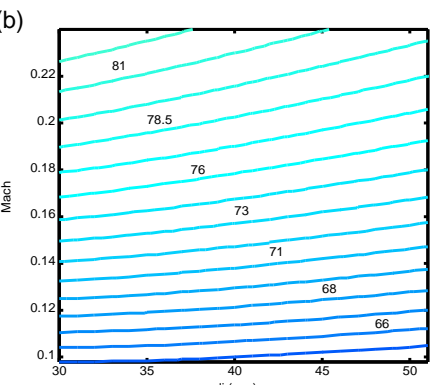

(d)

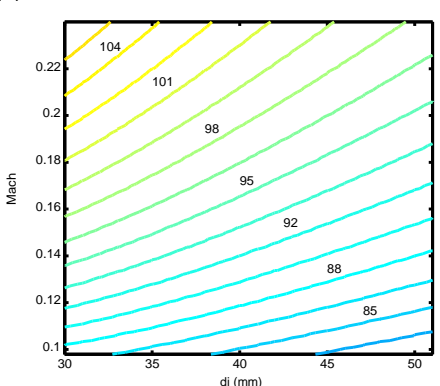

Figura 4.23: Evolución de la intensidad radiada y transmitida, en función del número de Mach y el diámetro de la cánula de entrada $d_{i}$ para las geometrías de $d_{o}=42 \mathrm{~mm}$, en el caso en que el resto de parámetros geométricos permanezcan invariables. Se estudia su aspecto tridimensional (figuras de la izquierda) y su proyección en los mapas de curvas de nivel reproducidas (figuras de la derecha).

consecuencia de realizar estas medidas a $12 d_{o}$ de la entrada de la cánula de salida, cuanto menor es $d_{o}$ este registro se realiza más cerca de este cambio de sección (generación de turbulencia) y por tanto no se puede asegurar que el flujo turbulento esté totalmente desarrollado en el punto de medida. Por otro lado, puede que las anisotropías de la fuente, esto es, que su desviación frente a una posición centrada en la cánula no sean detectadas por la elección de la medida de presión en una de las paredes de la cánula. Este segundo razonamiento se muestra claramente a través de los gráficos de coherencia $\gamma_{x y}$, figura 4.25 , obtenidos para la disposición de los micrófonos en una misma sección de la cánula de escape como se comentó en el capítulo anterior. 

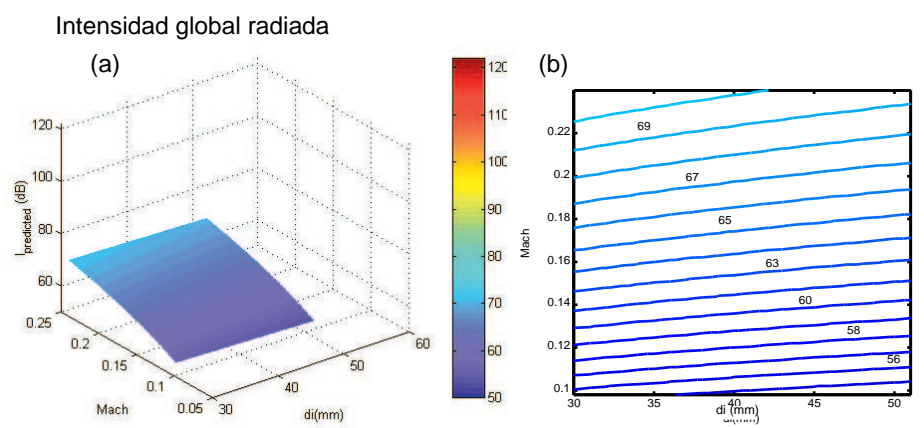

Intensidad global transmitida

(c)

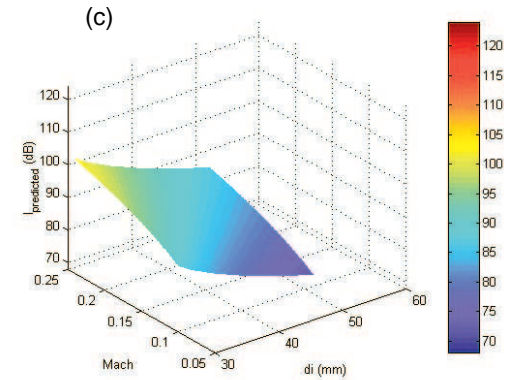

(d)

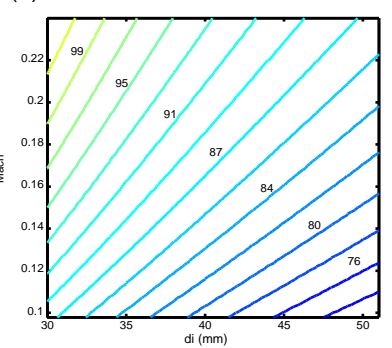

Figura 4.24: Evolución de la intensidad radiada y transmitida, en función del número de Mach y el diámetro de la cánula de entrada $d_{i}$ para las geometrías de $d_{o}=30 \mathrm{~mm}$, en el caso en que el resto de parámetros geométricos permanezcan invariables. Se estudia su aspecto tridimensional (figuras de la izquierda) y su proyección en los mapas de curvas de nivel reproducidas (figuras de la derecha).

Para la interpretación de estos gráficos se debe tener en cuenta la definición de la coherencia $\gamma_{x y}$ entre el registro de presión realizado por dos micrófonos $x$ e $y$, que corresponde a $\gamma_{x y}=\left|G_{x y}\right|^{2} /\left(G_{x x} \cdot G_{y y}\right)$, donde $G_{x x}$ y $G_{x y}$ representan el autoespectro y espectro cruzado del registro del micrófono $x$ con $y$, magnitudes definidas en el capítulo anterior. Por tanto, la coherencia $\gamma_{x y}$ representa la relación existente entre las señales registradas por los dos micrófonos, constituyéndose como un índice que cuantifica la validez de las medidas y determina cuándo las señales están correlacionadas (señal de presión transmitida) y no correlacionadas (turbulencia local) [134]. Dicho esto, podemos interpretar los resultados de la figura 4.25 del siguiente modo: para la geometría de diámetro de salida $d_{o}=51 \mathrm{~mm}(4.25(\mathrm{a}))$ se observan valores aceptables de la coherencia $\left(\gamma_{x y} \geq 0.6\right)$ para frecuencias inferiores a 3 $\mathrm{kHz}$, lo que se corresponde con una propagación mayoritaria a estas frecuencias de ondas planas acústicas, ya que no han comenzado a propagarse modos 

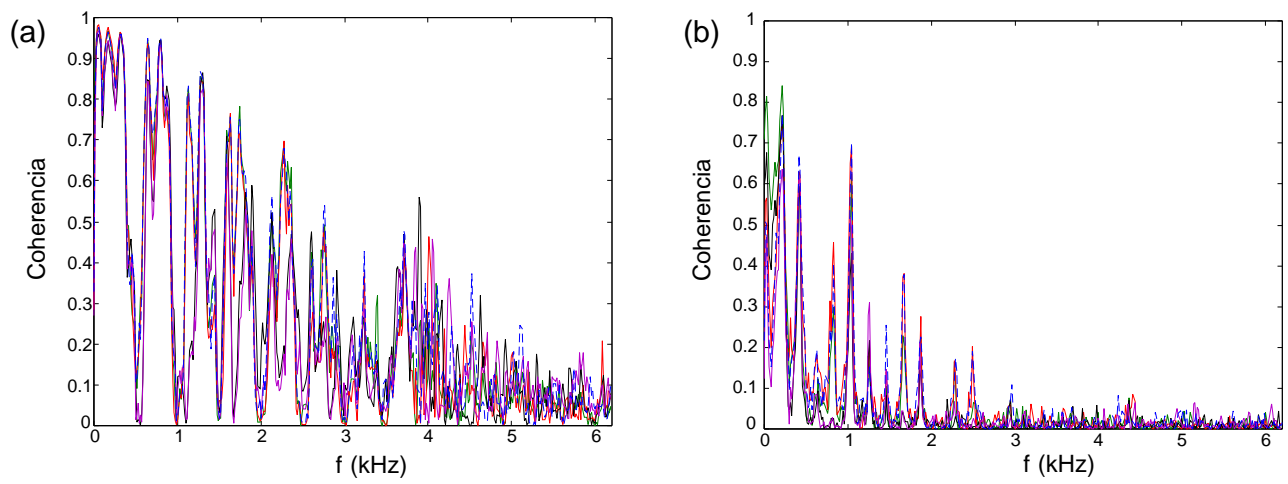

Figura 4.25: Representación de la coherencia entre los registros de presión realizado por los micrófonos situados en una misma sección de la cánula de salida, para un flujo de velocidad $U=68 \mathrm{~m} / \mathrm{s}$ para diferentes geometrías: (a) $d_{o}=51 \mathrm{~mm}$ y (b) $d_{o}=30$ $\mathrm{mm}$.

de orden superior, alejadas estas frecuencias de la turbulencia local del flujo. Sin embargo, para las geometrías de $d_{o}=30 \mathrm{~mm}$ esta situación cambia radicalmente al observarse una baja coherencia a partir de $1 \mathrm{kHz}$, lo que indica que la proporción de cada autoespectro con correlación espacial es pequeña en comparación con el nivel global de la señal, es decir, que las señales en esta posición de medida, a partir de estas frecuencias, están afectadas por la turbulencia local existente en esta sección, siendo imposible asegurar la obtención de valores fiables en este tipo de geometrías a través de la técnica de intensimetría en conductos para frecuencias superiores a $1 \mathrm{kHz}$, para el punto de medida escogido.

En relación a la posible anisotropía de la fuente emplazada a la entrada de la cánula de salida, pueden extraerse diversos resultados de la simulación numérica de flujos estacionarios mediante el empleo de códigos CFD. En la figura 4.26 se muestran estos resultados para dos longitudes de la cámara diferentes, observándose que las longitudes más cortas y de mayor intensidad de energía cinética turbulenta, poseen una fuente más centrada respecto a la entrada de la cánula de salida (4.26(b)), mientras que las cámaras de mayor longitud poseen menor intensidad de la energía cinética turbulenta en este punto pero esta fuente se encuentra situada en uno de los vértices de esta sección. Esto es, la posición de medida de presión en el interior de la cánula será un factor esencial, ya que el diferente comportamiento de la fuente puede hacer que la situación de los micrófonos en la pared de la cánula de salida, donde se produce el máximo de intensidad en las cámaras más largas, las 
muestren como fuentes más potentes a pesar de que los valores de energía cinética turbulenta muestren todo lo contrario.

Este efecto observado para los diámetros de salida pequeños se reproduce en las simulaciones tridimensionales tanto para $d_{o}=30 \mathrm{~mm}$ como para $42 \mathrm{~mm}$; sin embargo, el hecho de que no ocurra lo mismo para los diámetros de salida mayores $d_{o}=51 \mathrm{~mm}$, se debe a que en estas geometrías el comportamiento de las fuentes es radicalmente distinto, como se puede comprobar en la figura 4.27. Se observa para estos diámetros que el efecto del cambio de la longitud de la cámara tiene como consecuencia un cambio mucho más acusado en los valores de energía cinética a la entrada de la cánula de salida; sin producirse ningún cambio en la posición relativa del máximo de la energía cinética turbulenta $(\mathrm{k})$ en dicha sección, pero si una traslación, para las cámaras de mayor longitud, del valor máximo de $\mathrm{k}$ alcanzado en el silenciador (la fuente principal) hacia la salida de la cánula de entrada $(4.27(\mathrm{~b}))$, donde tiene lugar el fenómeno de separación del flujo.

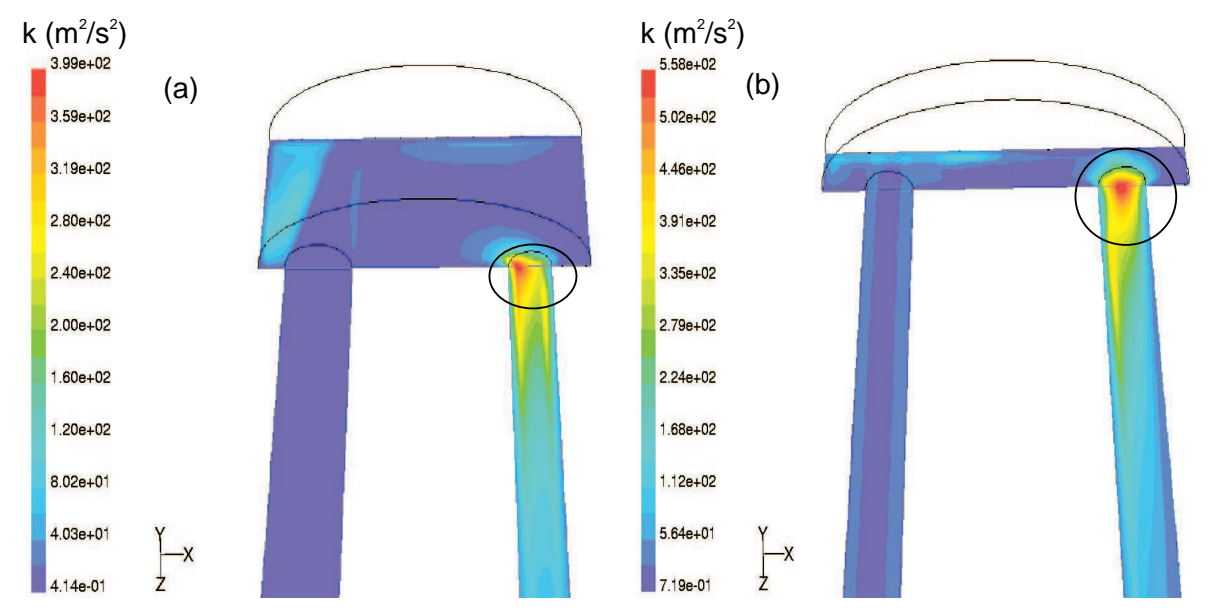

Figura 4.26: Energía cinética turbulenta $k$, simulada para una misma velocidad del flujo medio $(U=70 \mathrm{~m} / \mathrm{s})$ para dos longitudes de una cámara de expansión reversa diferentes con $d_{o}=30 \mathrm{~mm}$ en ambas: (a) $L_{c}=100 \mathrm{~mm}$ y (b) $L_{c}=25 \mathrm{~mm}$.

Como conclusión general de los resultados presentados cabe señalar que para poder extraer la máxima información de este método en el análisis del comportamiento de las fuentes internas del silenciador, es importante acompañar todos estos resultados experimentales por simulaciones numéricas estacionarias, como las mostradas en las figuras anteriores. 


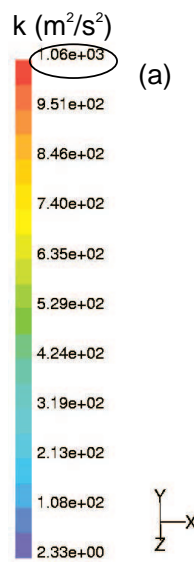

(a)

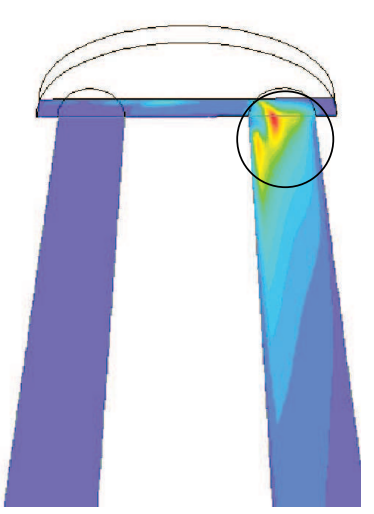

$\mathrm{k}\left(\mathrm{m}^{2} / \mathrm{s}^{2}\right)$

$1.70 \mathrm{e}+03$

$1.53 \mathrm{e}+03$

$1.36 e+03$

$1.19 \mathrm{e}+03$

$1.02 \mathrm{e}+03$

$8.53 e+02$

$6.830+02$

$5.14 e+02$

3.44e+02

$1.74 \mathbf{e}+02$

$3.54 \mathrm{e}+00 \quad \mathrm{X}$ (b)

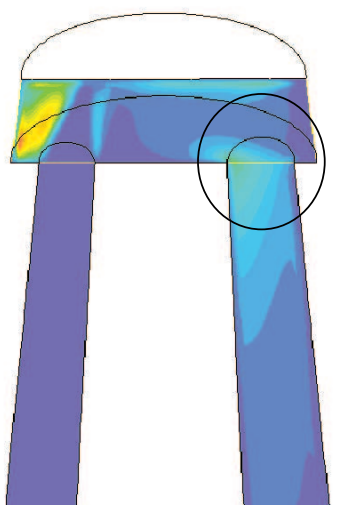

Figura 4.27: Energía cinética turbulenta $k$, simulada para una misma velocidad del flujo medio $(U=70 \mathrm{~m} / \mathrm{s})$ para dos longitudes de una cámara de expansión reversa diferentes con $d_{o}=51 \mathrm{~mm}$ en ambas: (a) $L_{c}=25 \mathrm{~mm}$ y (b) $L_{c}=100 \mathrm{~mm}$.

En relación a los criterios de diseño que se derivan de estas correlaciones deben mencionarse los siguientes:

- A pesar de que a simple vista todos los resultados mostrados parecen llevar a la conclusión de que se disminuye la intensidad sonora generada al reducir el diámetro de salida, esto se ha obtenido para rangos similares de velocidades medias del flujo saliente pero no de gastos medios, ya que es sabido que para un mismo gasto medio de entrada la velocidad de salida del flujo aumenta al disminuir el diámetro de la cánula.

- La obtención de leyes de escalado permite analizar los resultados que se obtendrían para un mismo gasto en las diferentes geometrías simplemente con sustituir su valor en la ley de escalado correspondiente. Siendo esta posibilidad de extrapolación en la predicción de la energía sonora generada por geometrías no medidas, una de las mayores ventajas que ofrece nuestra metodología.

- Por otro lado las principales tendencias extraídas con el objetivo de reducir la emisión sonora de ruido de flujo en la geometría considerada son:

1. Aumentar la longitud de la cámara.

2. Aumentar la separación de las cánulas.

3. Aumentar el diámetro de la cánula de entrada. 
Todos los estudios realizados a partir de las medidas de intensimetría en conductos la presentan como una técnica de medida poderosa y a partir de la cual se puede extrapolar una metodología adecuada para el estudio del ruido de flujo generado por los elementos del sistema de escape, proporcionando un mayor conocimiento de los mecanismos de generación interna, del comportamiento de la fuente y por lo tanto, aportando criterios de diseño de utilidad en la práctica. 



\section{Capítulo 5}

\section{Estudio computacional}

5.1 Introducción . . . . . . . . . . . . 179

5.2 Métodos computacionales: Usos y métodos . . . 180

5.2.1 Estudio computacional de las teorías acústicas . . 181

5.2.2 Simulación numérica directa . . . . . . . . . . . 183

5.2.2.1 Condiciones de contorno para fluidos no estacionarios . . . . . . . . . . 184

5.2.2.2 Selección del esquema de diferencias finítas 185

5.2.3 Simulación numérica directa más cálculo acústico . 186

5.2.4 Efectos turbulentos . . . . . . . . . . . . . . . 188

5.3 Simulaciones numéricas de los campos turbulentos 189

5.3.1 Determinación de campos de velocidades estocásticos 189

5.3.2 Modelos de turbulencia de dos escalas . . . . . . . 191

5.3.3 Representación de flujos turbulentos mediante la generación de señales no gausianas . . . . . . . . . 192

5.4 Cálculos acústicos mediante métodos numéricos . 193

5.5 Estudio computacional del ruido de flujo generado por silenciadores . . . . . . . . . 197

5.5.1 Cálculo de la presión acústica radiada . . . . . . . 200

5.5.2 Estimación de la intensidad sonora global transmitida al campo libre . . . . . . . . . . . . 206

5.6 Resultados de la aplicación a una geometría simple207 



\subsection{Introducción}

Como se ha mencionado en el capítulo anterior, el análisis realizado de las aproximaciones 'puramente teóricas' en aeroacústica se debe complementar con una revisión de los principales problemas en la investigación experimental (realizado en el capítulo previo), y computacional de los fenómenos aeroacústicos claves. Como se ha podido observar cualitativamente en capítulos precedentes, a través de los resultados obtenidos con cálculos CFD mediante el código comercial FLUENT, los códigos computacionales proporcionan una nueva herramienta para la comprensión de los mecanismos físicos asociados a los problemas de generación sonora por diferentes flujos. En la actualidad, y debido a que las ondas sonoras se caracterizan por poseer amplitudes relativamente pequeñas en comparación con las variables de flujo de fondo, el caballo de batalla de multitud de códigos computacionales radica en resolver los problemas relevantes de la generación sonora con mayor precisión.

Este campo de estudios aeroacústicos es el que presenta mayor actividad en la actualidad, dando por sentado que quizás el trabajo teórico ya fue realizado en el pasado y que ahora es el momento de analizar, validar y expandir estos análisis teóricos con herramientas computacionales. Particularmente, nosotros en este capítulo pretendemos ir más allá en el empleo de este tipo de códigos computacionales intentando obtener valores cuantitativos de las emisiones de ruido de flujo generado en el interior de silenciadores, de los que se dispone de medidas experimentales. Ya que, la finalidad es desarrollar una metodología de estudio del ruido de flujo lo más potente posible que englobe estudios experimentales y computacionales, de acuerdo con los comentarios de Crocker [33]: "en los casos en que la geometría del espacio acústico es complicada y donde todos los elementos de aproximación teórica no pueden ser empleados, es necesario usar aproximaciones numéricas".

Actualmente existe una extensa literatura sobre la propagación sonora en sistemas de escapes [46] y softwares asociados a su predicción, sin embargo ninguno de ellos incluye predicciones cuantitativas de la contribución del ruido de flujo generado y la predicción de su emisión sonora.

En este capítulo se realiza, en primer lugar, una somera revisión de algunos de los trabajos computacionales recogidos en la literatura científica, los cuales han sido desarrollados con la intención de confirmar la validez de las aproximaciones a la teoría de sonido aerodinámico comentadas en el capítulo anterior. En este sentido, es digno de mencionar la necesidad de desarrollar modelos matemáticos más eficientes y algoritmos para la simulación numérica de los fenómenos aeroacústicos menos estudiados, particularmente los proce- 
sos de generación sonora por flujos altamente inestables, ya que la mayoría de los métodos computacionales actuales son imperfectos. Con esta motivación se desarrollará una formulación matemática aproximada para obtener el valor del ruido de flujo emitido por silenciadores, construyendo un campo de velocidades fluctuantes en el interior de un silenciador comercial e introduciendo la expresión global, desarrollada, de la analogía acústica de Lighthill, así como las contribuciones del sonido generado por la vorticidad que contienen las teorías de Powell-Howe, para obtener finalmente un valor de intensidad global emitida a un punto del espacio. Magnitud de la que se dispone de valores experimentales, lo que nos permitirá validar la metodología de simulación y cálculo propuesta. Todo ello nos servirá de base para elaborar criterios de diseño.

\subsection{Métodos computacionales: Usos y métodos}

La aplicación de los métodos computacionales modernos proporciona una considerable contribución a la comprensión de los fenómenos aeroacústicos. Actualmente, se sugieren multitud de aproximaciones computacionales para la simulación de flujos gaseosos no estacionarios, y la aeroacústica computacional se ha constituido como un área de estudio separada de la aerodinámica computacional. Por contrapartida, sólo algunas de estas aproximaciones pueden ser aplicadas para la resolución de problemas aeroacústicos complejos, y menos, para el análisis de la generación sonora en flujos altamente inestables. En esta sección, y debido a la ilimitada longitud que esto supondría, no se realizará una revisión detallada del estado actual de la aeroacústica computacional, lo que incluiría la comparación de los métodos más populares y la consideración de muchas soluciones definidas con el análisis de los errores principales en cada caso, únicamente se mencionarán brevemente algunas de las referencias claves.

Tal como mencionan Wells y Renaut [175] el mayor avance en el análisis acústico se ha producido gracias a la disponibilidad de los recursos computacionales actuales. La aerodinámica y otras áreas de la mecánica de fluidos se han beneficiado inmensamente del desarrollo del campo conocido como 'Computational Fluid Dynamics' (CFD). Tanto el avance en las técnicas numéricas como el de las máquinas de cálculo han hecho posible el análisis numérico de los flujos, y su aplicación al análisis de los problemas relacionados con la aeroacústica. En este sentido se debe hablar de la aeroacústica computacional y no únicamente de métodos computacionales, campo en el cual la mayor parte de los esfuerzos actuales conciernen al desarrollo de esquemas de aproximación de diferencias finitas a fin de que se conserve la física de la propagación de onda. 
Por otro lado, se debe reconocer que los métodos CFD han permitido realizar grandes progresos especialmente en diseños aerodinámicos y de aviones, pero su aplicación a resolver problemas aeroacústicos presenta algunas limitaciones, ya que se debe tener en cuenta que la naturaleza, características y objetivos de los problemas aeroacústicos, es diferente de los problemas aerodinámicos generales (Tam [162]). Así por ejemplo, los problemas aerodinámicos son, normalmente, independientes del tiempo, mientras que los problemas aeroacústicos son, por definición, dependientes del tiempo.

Quizás el área más desarrollada sea el estudio computacional de la propagación sonora en flujos subsónicos y estacionarios, tanto internos (en conductos) como libres. En este campo hay acumulada mucha experiencia en la solución de las ecuaciones lineales de pequeñas perturbaciones, lo cual no significa que no surjan muchas dificultades, como por ejemplo, ante el problema de la introducción de fuentes sonoras en el volumen generadas por el mismo flujo [63].

Revisiones de este tema, como por ejemplo la de Wells y Renaut [175] y la de Ffowcs Williams [64], revelan la existencia de códigos computacionales aplicados para la predicción de la emisión de sonido aerodinámico basados en algunas de las aproximaciones teóricas analizadas en el capítulo anterior, pero debido a los defectos que poseen, surgen dudas sobre la validez de sus resultados [63].

Se debe tener en cuenta, por otro lado, que en la mayor parte de los estudios aeroacústicos completos hay un acoplamiento inherente entre la parte aerodinámica del problema y la acústica, como indican Wells y Renaut [175]; consecuentemente, gran cantidad de los esfuerzos actuales giran en torno al cálculo de la interacción de la onda acústica con las estructuras del flujo tales como estelas, vórtices, etc.

\subsubsection{Estudio computacional de las teorías acústicas}

En primer lugar, cabe citar la discusión realizada por Wells y Renaut [175] sobre el análisis de los problemas asociados al empleo de procedimientos estándars de la aerodinámica computacional para los cálculos acústicos siguiendo las ideas de la analogía acústica introducida por Lighthill. Estos cálculos acústicos emplean en la mayoría de los casos las superficies aerodinámicas en movimiento introducidas por Ffowcs Williams y Hawkings, considerada por diversos autores como la forma más general de la analogía acústica de Lighthi1l. Otros autores optan por el uso del teorema de Kirchhoff para la predicción de los campos sonoros generados en campo libre, tal como se muestra en las 
referencias $[66,111,117]$, siendo particularmente interesante el trabajo presentado por Hariharan et al. [72]. Predicción que a menudo está basada en una cierta solución CFD obtenida previamente en un dominio finito con un flujo altamente inestable.

La forma más general de estas aproximaciones contiene dos etapas diferenciadas. En primer lugar, en una fase de simulación numérica directa DNS (Direct Numerical Simulation) se obtiene una solución calculada para un cierto flujo inestable a través de la integración de las ecuaciones de Navier-Stokes o de Euler (normalmente, en términos de variables totales). Generalmente, este sistema puede ser empleado para medios compresibles, pero también puede aplicarse para modelos de flujo incompresible en el caso de flujos subsónicos, como presentan Wang et al. [174].

En un paso siguiente, esta fase es tomada como una aproximación del flujo de fondo, empleándose posteriormente alguna versión de la analogía acústica de Lighthill, o formas modificadas como las de Powell o Howe, etc., para estimar la 'fuente sonora' $(q)$ y, a su vez, el ruido en el campo lejano. La elección de una teoría u otra, dependerá de entre otras cosas del número de Mach con que se trabaje, lo que permitirá en algunos casos el empleo de formas simplificadas de las mismas. En este sentido, aparte de la forma de la analogía original derivada por Lighthill, las formulaciones de Powell y Howe han sido aplicadas extensivamente, incluso la versión de Möhring de esta aproximación ha sido empleada en algunos trabajos [63]. Todos los progresos realizados en la dirección computacional son, en general, apreciados como bastante exitosos, a pesar de que autores como Wells y Renaut [175] manifiestan una crítica cautelosa, como la que se desprende de la siguiente afirmación: "aunque la analogía acústica se ha verificado como muy poderosa para la predicción acústica, para muchos de los problemas de interés no parece ser conveniente la formulación en términos de regiones separadas de fuentes y propagación".

Como método alternativo, algunos autores han planteado la posibilidad de emplear ecuaciones no lineales para las perturbaciones no estacionarias sobre flujo medio de fondo estacionario, las cuales se habían calculado anteriormente con un algoritmo separado. Desafortunadamente, tal como comenta Fedorchenko [63], estas perturbaciones suelen ser tratadas como sonido, aunque de hecho esto incluye todo tipo de ondas, no únicamente de sonido.

Otra forma de estudiar la forma no lineal de un sistema, abordándolo mediante un cálculo directo sin emplear ninguno de los métodos numéricos propuestos, es el propuesto por Mast y Pierce [113], quienes tratan un sistema de resonador con flujo como un sistema no lineal autónomo. En él se encuentran los ciclos límite del sistema mediante el análisis de la función de 
descripción, en la cual cada componente del sistema de oscilación no lineal está representada por una función de respuesta frecuencial asociada. El método de análisis de la función de descripción, es un método por el cual un oscilador no lineal puede modelarse como un grupo de elementos acoplados, donde como ya se dijo antes, cada elemento está representado por la función de respuesta frecuencial asociada. Esta técnica es comúnmente usada en la teoría de control para calcular los ciclos límite de un sistema no lineal.

En la actualidad se emplean multitud de estos métodos de simulación directa, DNS, para resolver los problemas específicos derivados del estudio computacional de flujos supersónicos, incluyendo, claro está, el estudio de chorros supersónicos, como el realizado por Viswanathan y Sankar [173], donde los efectos fuertemente no lineales son cruciales en los procesos de generación sonora. Por tanto, en estos casos el uso de sistemas completamente no lineales de las ecuaciones de Navier Stokes o Euler se constituyen como los más prometedores para obtener una solución de DNS. Por otro lado, los modelos 'tradicionales' de fuentes sonoras aerodinámicas, la mayoría de los cuales están basados en la analogía de Lighthill, suelen proponerse para este tipo de problemas, proporcionando resultados satisfactorios, como se recoge en los trabajos de Bastin et al. [9]. Los problemas y ventajas que presenten estos modelos se escapa de nuestro campo de investigación (aplicación del ruido de flujo turbulento emitido en los procesos de escape por motores de combustión interna alternativos), por lo que en esta revisión no se hará mención explícita de ellos.

\subsubsection{Simulación numérica directa}

Como se ha mencionado anteriormente en los cálculos aeroacústicos se opta generalmente por realizar una primera fase de simulación numérica directa (DNS) con la finalidad de obtener una primera solución del flujo inestable. En la aplicación al cálculo aeroacústico para números de Mach pequeños a través de DNS, Crighton señala que la aproximación sonora se encuentra con una serie de dificultades entre las cuales se incluyen: (i) la gran disparidad existente entre la longitud de la escala acústica y la longitud de escala integral de la turbulencia [31], (ii) la menor contribución de la energía turbulenta a la energía radiada al campo acústico lejano y (iii) la estructura multipolar de la fuente acústica básica [153]. Por estas razones, para el cálculo del sonido radiado por flujos de bajos números de Mach es recomendable el uso de una analogía acústica. 
Por otro lado, para obtener una solución válida del campo de flujo inéstable que gobierna la generación sonora tenemos que tener en cuenta algunas de las limitaciones que poseen los modelos computacionales existentes, las cuales son resumidas a continuación.

\subsubsection{Condiciones de contorno para fluidos no estacionarios}

En el caso de flujos no estacionarios, el hecho de no tener una condición de contorno constante en el tiempo, puede constituir un problema importante. Para resolver esta cuestión, puede optarse por la integración de los sistemas generales no lineales de Navier-Stokes o Euler para las variables totales que gobiernan el movimiento del medio compresible. Esta aproximación, que puede clasificarse como 'una simulación numérica directa de un flujo compresible inestable' (DNSC) [63], parece ser adecuada para la simulación de cualquier fenómeno acústico no lineal. A pesar de algunas restricciones, el disponer de una DNSC posee una importante ventaja frente a los sistemas de medidas experimentales actuales, ya que mediante él, puede obtenerse todo el conjunto de variables del flujo en cada punto y para cada instante de tiempo.

Otro modo de resolución del problema de la condición de contorno es escoger condiciones de contorno que sean permeables a una variación temporal (aproximaciones que implican un análisis continuo de los fenómenos durante la búsqueda de la solución [63]), o construir una condición de contorno variable con el tiempo mediante el movimiento de superficies. Así, por ejemplo, Kim y Lee [90], simulan la carrera de admisión de un motor mediante un modelo axisimétrico empleando las ecuaciones no estacionarias y compresibles de Navier-Stokes, y considerando el movimiento de un pistón simple como válvula de admisión. Con este simple modelo geométrico se modela la presión durante la carrera de admisión, que genera una onda cerca de la válvula de admisión justo cuando ésta se cierra, la cual además tiene un comportamiento no lineal y se propaga y radia con una amplitud relativamente grande.

Los modelos computacionales de condiciones de contorno para el flujo incidente y flujo saliente persiguen el objetivo de minimizar los efectos de reflexión sonora (esto es, por ejemplo, condiciones de absorción sonora). Los modelos usuales de contornos no-reflectantes son desarrollados dentro de una ecuación hiperbólica lineal simple para una variable escalar o por consideración de la versión linealizada del sistema de Euler. Desafortunadamente, estas características basadas en las condiciones de contorno pueden funcionar bien únicamente para ondas sonoras que tienen una incidencia aproximadamente perpendicular al contorno. En relación a este problema Gloerfelt et al. [70] 
plantean una solución alternativa, empleando una formulación asintótica de las ecuaciones de Euler en las condiciones de radiación y de salida del fluido. De esta forma se considera una zona 'esponja' que permita la salida de las estructuras vorticales sin producir una reflexión notable. De esto se desprende que la especificación de un sistema de condiciones de contorno apropiado, el cual debe ser no local en el espacio y en el tiempo, representa un problema crucial en la aeroacústica computacional.

\subsubsection{Selección del esquema de diferencias finítas}

Otro problema clave es la selección de un adecuado esquema de diferencias finitas, ya que los fenómenos que normalmente se deben resolver se caracterizan por poseer pequeñas amplitudes de fluctuación sonora, siendo este esquema una posible fuente de error, como comenta Tam [162], ya que una mala elección puede distorsionar el cálculo del flujo medio y proporcionar una gran disparidad entre las variables acústicas y las de flujo. El esquema apropiado deberá dar solución al amplio rango de frecuencias sonoras y empleará por tanto una malla con el mínimo tamaño de celda posible, que debe ser menor que la mínima longitud de onda sonora que se quiera considerar $[71,86]$. Además de esto, se imponen algunas restricciones y requerimientos específicos a la estructura espacialmente no homogénea de la malla que cubre el dominio computacional finito; como por ejemplo, el evitar importantes extensiones de ésta a lo largo de algunas direcciones, ya que esto podría causar efectos ficticios de absorción, reflexión y refracción en los campos sonoros [25].

A pesar de que los procesos de generación sonora por un flujo son esencialmente no lineales, el orden de precisión de un esquema se estima normalmente dentro de un sistema de ecuaciones diferenciales simplificado, a menudo linealizado, ya que generalmente no es posible realizar esto dentro de un sistema completamente no lineal de las ecuaciones de mecánica de fluidos; razón por la que muchos de los esquemas frecuentemente aplicados son incapaces de proporcionar un alto orden de precisión en la aproximación a las ecuaciones no-lineales más relevantes.

En el proceso de propagación sonora es importante considerar, entre otros factores, la posible anisotropía espacial que puede distorsionar este proceso; esto es especialmente importante cuando se simulan flujos susbsónicos. En este sentido se han destinado grandes esfuerzos a minimizar los efectos espúreos tanto de la anisotropía, como de la dispersión y disipación en el esquema considerado, lo que permitiría amoldarse mejor a la física real de la propagación sonora a grandes distancias. A este respecto, Tam [162] afirma 
que para asegurar que la solución calculada sea adecuada a grandes distancias de propagación, el esquema numérico debe estar prácticamente libre de dispersión numérica (grandes diferencias entre el dominio computacional de la fuente sonora y su entorno), de disipación y de anisotropía. Estas tres anomalías directas: amplitud de disipación, la reflexión parásita de alta frecuencia espacial debida a los modos de propagación de la onda y la dispersión de la forma de la onda, representan probablemente las mayores barreras a la solución numérica de los problemas aeroacústicos, donde se buscan soluciones a grandes distancias de la fuente inicial; lo que sin embargo, es fácil de lograr si se consideran un gran número de puntos de mallado por longitud de onda.

Por otro lado, muchos de los flujos reales son demasiado complicados para ser simulados con las aproximaciones estándars existentes. Por ejemplo, para un flujo subsónico inestable todos los defectos locales del modelo computacional, tanto en el contorno como en el volumen, pueden tener una influencia importante en la evolución del flujo en el dominio espacial completo, debido a la interconexión global existente a través de las ondas sonoras, y por tanto, puede obtenerse una solución global no válida. Esto sugiere la necesidad de aplicar las aproximaciones más precisas que sea posible, tanto teóricas como computacionales.

Sí se considera un método de diferencias finitas basado en un sistema no lineal de Navier-Stokes o Euler para un medio compresible, este es potencialmente capaz de resolver todo tipo de procesos de ondas, incluyendo el sonido, siendo en general, sencillo separar el flujo inestable de fondo (incluyendo las perturbaciones convectivas de entropía y vorticidad) obtenido de una solución computacional completa para las variables totales, como muestra Fedorchenko en diferentes estudios [63]. Por el contrario, es muy dificil, algunas veces prácticamente imposible, extraer las perturbaciones acústicas a partir de la solución general, en especial para flujos internos disgregados, lo cual ya constituía un problema experimental. Sin embargo este método puede lograr grandes éxitos aplicado a numerosos problemas aeroacústicos no lineales.

\subsubsection{Simulación numérica directa más cálculo acústico}

Como resultado del esfuerzo por confirmar la validez de la analogía acústica de Lighthill se ha extendido el uso de la DNS más un cálculo acústico, como muestran los recientes trabajos de Mitchell et al. [117]. Mediante la aplicación de esta aproximación se obtuvo el sonido generado por un par de vórtices en una capa de mezcla bidimensional compresible (Colonius [28]), basado en una solución numérica directa de las ecuaciones compresibles de 
Navier-Stokes en un dominio computacional que incluía tanto una región de campo próximo como una porción del campo acústico (campo lejano).

Por otra parte, el trabajo de Mitchell, citado anteriormente [117], analiza la posible aplicación de diferentes métodos para la estimación del campo sonoro radiado, estableciendo una comparación entre el campo sonoro radiado obtenido directamente como solución de DNSC (sin emplear ninguna teoría acústica), con las predicciones obtenidas por la solución de la ecuación de Lighthill [102], y el calculado empleando el método de Kirchhoff aplicado a una cierta superficie de integración localizada en el interior del dominio computacional. Este trabajo concluye señalando que las predicciones obtenidas mediante ambos métodos muestran un buen acuerdo con los datos calculados directamente (DNSC), subrayando que la elección del método de predicción depende de los datos de entrada procedentes del cálculo computacional, de forma que es importante tratar explícitamente los términos fuente en la ecuación de Lighthill y escoger una superficie de integración apropiada en la aproximación de Kirchhoff.

También cabe citar que autores como Mu y Mahalingam [123], emplearon la DNS para el estudio de la interacción de unas perturbaciones acústicas impuestas monocromáticas y dependientes del tiempo, con un flujo medio de corte estacionario en un conducto bidimensional. Los resultados numéricos mostraban buenos acuerdos con las predicciones analíticas obtenidas por otros autores.

Otros autores como Sarkar y Hussain [153] emplean un modelo híbrido para el cálculo numérico de la radiación acústica a partir de una turbulencia isótropa. En él, se combinan la DNS del flujo turbulento con la analogía acústica de Lighthill. En este caso la eficiencia acústica de la turbulencia isótropa simulada parece ser sustancialmente menor que la hallada en experimentos con chorros subsónicos. Por otro lado, la frecuencia dominante de la presión acústica calculada se encontró algo mayor que la frecuencia dominante de la energía contenida en las escalas de movimiento. La potencia acústica obtenida en las simulaciones se mostró que era proporcional a $\varepsilon M_{t}^{5}$, donde $\varepsilon$ es la velocidad de disipación turbulenta y $M_{t}=\sqrt{\overline{u_{i}^{\prime} u_{i}^{\prime}}} / c$ es el número de Mach turbulento, donde $c$ es la velocidad del sonido y $u_{i}^{\prime}$ son las componentes de las velocidades del flujo turbulento, lo cual coincide con los resultados analíticos obtenidos por Proudman, como queda recogido por Morse [121], aunque la constante de proporcionalidad es menor. 


\subsubsection{Efectos turbulentos}

La complejidad que plantea el resolver el problema de las interacciones no lineales entre los movimientos vorticales a gran escala y la pequeña escala turbulenta, interacciones que pueden influenciar de manera importante los mecanismos de generación sonora, afecta a las exigencias de los códigos computacionales. El método DNSC parece inapropiado para la solución de estos problemas específicos, tanto para la simulación directa de la turbulencia como para flujos reales de interés práctico en ingeniería o geofísica [99], ya que, los tamaños prácticos de las mallas espaciales son incapaces de facilitar la simulación de las pequeñas escalas de la turbulencia, basadas en las ecuaciones de Navier-Stokes o Euler. Así, por ejemplo, Radavich et al. [146] emplean métodos CFD para modelar la interacción compleja que ocurre entre el flujo y las resonancias acústicas, producidas en un resonador de cuarto de onda a bajos números de Mach, a través de la resolución de ecuaciones de Navier-Stokes compresibles, turbulentas y no estacionarias.

En este contexto se han desarrollado diversos métodos computacionales para la simulación de movimientos inestables a gran escala, en medios compresibles e incompresibles, donde se tiene en cuenta la evolución de estas escalas mediante el uso de algunos modelos adicionales. Estas aproximaciones son denominadas "large eddy simulation" (LES). Algunos autores, como Bastin et al., han proporcionado algunas versiones específicas de estos modelos de turbulencia, así, por ejemplo estos autores [9] emplean el modelo clásico $\mathrm{k}-\varepsilon$, como un modelo semideterminista de la turbulencia (SDM) donde son evaluadas únicamente las grandes escalas coherentes del movimiento. Otro autores como Lo et al. [108] emplean la LES de las ecuaciones de Navier-Stokes compresibles, para investigar y representar mediante animaciones dinámicas las estructuras vorticales generadas en un chorro plano.

Evidentemente, si el tamaño de la malla tiende hacia cero, la LES se transforma en una DNS. Cuando es obtenida una cierta solución para estos movimientos a gran escala en un dominio finito de cálculo, la principal meta es estimar la emisión sonora, relevante, en el campo lejano (a distancias que son mucho mayores que el tamaño característico de dicho dominio). Algunos autores intentan hacer esto empleando alguna versión del método de Kirchhoff o a través de la analogía acústica de Lighthill, de modo similar a como se emplea la DNS, para la que se han presentado algunos ejemplos en la sección anterior.

Otro modo de aplicación de la LES para el cálculo directo del ruido generado por flujos turbulentos es el empleo de métodos híbridos tal como sugieren Bogey et al. [19], que permiten salvar el problema de la diferencia de 
escalas entre el campo aerodinámico y los campos acústicos. En esta aproximación híbrida se emplean las ecuaciones de Euler linealizadas (EEL) como un operador de onda, mientras que la fuente acústica se obtiene por sustitución del campo de velocidad fluctuante aerodinámico calculado por un método de LES. El campo sonoro radiado determinado de esta forma coincide con el cálculo directo de este campo obtenido por la LES, lo que permite validar la analogía acústica basada en las EEL, tanto para el modelo del término fuente utilizado, como para los efectos del flujo medio en la propagación sonora.

La DNS y la LES son herramientas importantes para la comprensión de la física de flujos turbulentos y los mecanismos por los cuales se genera el ruido, como ya se mencionó anteriormente, pero están restringidas en la práctica al caso de geometrías simples, y en el caso de DNS para bajos números de Reynolds, no siendo utilizadas en casos como por ejemplo el de emisión de chorros por toberas. Además, el cálculo de la propagación de la onda acústica en un medio espacial y temporalmente inhomogéneo (como es el caso del campo de flujo turbulento), así como el sonido generado, son problemas no triviales que requieren altos niveles de precisión para reproducir con garantías la relación de fase entre las ondas.

\subsection{Simulaciones numéricas de los campos turbu- lentos}

Una vez han sido comentados los procesos y los pasos seguidos para abordar la resolución de fenómenos aeroacústicos mediante métodos numéricos computacionales, y mencionados los problemas asociados a estos estudios, parece adecuado realizar un breve resumen de algunos de los modelos de simulación de los campos turbulentos que existen en la actualidad, ya que es este campo el que se encuentra íntimamente relacionado con el término fuente del proceso de generación sonora, cuya importancia ya ha sido manifestada en el capítulo anterior.

\subsubsection{Determinación de campos de velocidades estocásticos}

En esta aproximación, propuesta por Béchara et al. [11], las ecuaciones que gobiernan el campo sonoro son tratadas en dos pasos. En la primera etapa se estudian las componentes medias y turbulentas del flujo mientras que las perturbaciones acústicas son despreciadas. En un segundo paso se derivan un conjunto de ecuaciones para las variables acústicas del proceso. 
El sistema de ecuaciones de movimiento planteado, contiene en el miembro de la izquierda las ecuaciones de Euler linealizadas (Bailly et al. [5]), y en el miembro de la derecha el término fuente relacionado con las fluctuaciones turbulentas y sus interacciones con el flujo medio. Estos términos son modelados empleando una descripción estocástica del movimiento turbulento tridimensional, ya que estos autores sostienen que el tensor de esfuerzos de Lighthill $T_{i j}$ es estocástico y puede definirse únicamente en un sentido estadístico. Esta descripción estocástica se logra sintetizando el campo de velocidad en cada punto del espacio y para cualquier instante de tiempo como una colección de modos discretos de Fourier. El campo derivado de esta forma posee momentos estadísticos espaciales adecuados y una densidad de potencia espectral razonable. También permite obtener señales de presión y patrones de campos sonoros realistas.

El término fuente necesario para realizar este cálculo se obtiene a través de la aplicación del modelo estocástico de generación y radiación sonora (SNRG). Este método [6] emplea representaciones estadísticas de la fuente, combinadas con un modelo de cierre turbulento como son las ecuaciones de transporte $\mathrm{k}-\varepsilon$, que se emplearán posteriormente para predecir el campo acústico radiado, por flujos turbulentos complejos, al campo lejano. Las líneas en que se basa el método de simulación del campo de velocidad estocástico espaciotemporal ideado por Bailly et al. [5] se encuentran resumidas en un trabajo de investigación realizado en esta Universidad [3], por lo que no se incluye en esta sección con el fin de no sobrecargarla con información prescindible para la compresión del modelo de cálculo propuesto.

El flujo se subdivide en una colección de regiones fuente independientes, con dimensiones longitudinales y laterales definidas por las escalas de correlación local de la turbulencia. Esta representación posee muchas características de la turbulencia real, pero no es perfecta, ya que aunque sí que exhibe la longitud de correlación esperada y, en particular, la razón de las escalas de longitud requerida, no hay rasgos de las propiedades convectivas del flujo de cizalla de la turbulencia. Razón por la que no se constituye como un modelo definitivo de la radiación sonora aerodinámica.

Este modelo de simulación turbulenta se ha empleado para resolver diversos problemas como la predicción del ruido radiado por flujos subsónicos, supersónicos [6], y por chorros libres [12], comparándose los resultados obtenidos con varias teorías acústicas, la de Ribner, la de Goldstein-Howe, etc.

Las limitaciones que presenta este modelo, en palabras de sus autores [11], radican en las propiedades estadísticas del campo turbulento sintetizado y en el uso de un modelo axisimétrico de propagación acústica para 
calcular el campo radiado. Esta última aproximación se fundamenta en la limitación que conlleva el intervalo temporal de cálculo, derivado de la necesidad de un método computacional tridimensional inicial para propagar las ondas sonoras desde la fuente de ruido, lo que requeriría una gran malla y una carga computacional inabordable. Así, el modelo escogido se reduce a un cálculo bidimensional axisimétrico, que permite considerar los efectos de refracción, que tienen influencia en la radiación espacial de las ondas acústicas desde la fuente sonora.

\subsubsection{Modelos de turbulencia de dos escalas}

Uno de los modelos de turbulencia más empleados para el cierre del sistema de ecuaciones de Navier-Stokes o de Euler es el modelo de turbulencia de dos ecuaciones (modelos en los que la viscosidad turbulenta $\mu_{t}$ se calcula mediante dos ecuaciones de transporte). Sin embargo, a pesar de los remarcables éxitos que presentan estos modelos en la predicción de flujos turbulentos, estos poseen importantes defectos, como es por ejemplo, la relación lineal, en términos de la viscosidad turbulenta en un vórtice, entre la tensión de Reynolds y la tasa de corte media, relación inadecuada en muchos problemas, como el caso de fluidos no-newtonianos. Sin embargo, en el caso particular de un fluido como el aire estos efectos no aparecen y por tanto esta aproximación resulta válida, esto nos lleva a que en el método de cálculo propuesto en este capítulo para la estimación del ruido de flujo generado por un silenciador, se escoga un modelo de este tipo, $\mathrm{k}-\varepsilon$ estándar, en el que la viscosidad turbulenta es obtenida como función de dos magnitudes: (i) la energía cinética turbulenta $\mathrm{k}$, y (ii) la velocidad de disipación turbulenta $\varepsilon$, magnitudes que responden a dos ecuaciones de transporte diferentes (como ya se verá más adelante).

En cualquier caso, es interesante comentar la solución que propuso Rubinstein [150] para evitar este defecto de los modelos de turbulencia de dos ecuaciones. Para ello, desarrolló un modelo de turbulencia de dos escalas partiendo de una formulación de modelos de turbulencia de múltiples escalas (en los cuales las ecuaciones de transporte para la energía cinética turbulenta y razón de disipación son reemplazadas, cada una, por ecuaciones de transporte de la energía cinética y razón de disipación concernientes a un rango definido de escalas del movimiento) y reduciéndolo a un modelo de dos ecuaciones cuando decae la turbulencia. La meta de este trabajo era derivar un modelo de dos escalas en el que la transferencia de energía y la disipación pudieran ser distintas y satisfacer diferentes ecuaciones de transporte, de forma que el rango inercial de transferencia de energía y velocidad de disipación puedan ser desiguales, empleando, para separar estos efectos, los principios de localidad y de transferencia inercial en la escala de Kolmogorov. 


\subsubsection{Representación de flujos turbulentos mediante la gene- ración de señales no gausianas}

Mengali y Micheli [115] proponen una técnica para la simulación de un flujo turbulento a la salida de un conducto. Esta técnica se basa en un banco de filtros lineales diseñados de tal modo que su respuesta impulsional cumple las propiedades espectrales de la señal que se desea generar, imponiendo a la entrada del banco de filtros ruido blanco no gausiano. Las señales generadas de esta forma pueden emplearse para resolver varios problemas de ingeniería tales como, verificación de la adecuación de los métodos de medida basados en anemometría de hilo caliente o obtención de ráfagas de componentes de velocidad indicadas para las aplicaciones en simuladores de vuelo.

El interés de poder simular y generar señales que representen de forma 'realista' las componentes de velocidad relacionadas con flujos turbulentos es uno de los requisitos más importantes en problemas como los de aeronáutica. Desafortunadamente, las aproximaciones clásicas para generación de señales con características espectrales específicas recurren a filtros lineales alimentados con ruidos blancos Gausianos, lo cual no es adecuado para las aplicaciones mencionadas, ya que el proceso que se quiere simular es típicamente no Gausiano. Así por ejemplo, para las variaciones temporales de las componentes de velocidad en un flujo turbulento, estos métodos clásicos proporcionaban resultados que en muchos casos diferían de los resultados experimentales de tal modo que las señales generadas exhibían momentos de tercer orden igual a cero y momentos de cuarto orden con relaciones semejantes a las de segundo orden. Con el objeto de dar respuesta a estos problemas surgieron nuevos modelos de simulación, en particular se intentó modelar la turbulencia atmosférica para simuladores de vuelo considerando un sistema no lineal compuesto por tres filtros lineales simulados por señales Gausianas con los que se obtenía cada componente de velocidad [115]. Este método tenía el inconveniente de falta de control sobre magnitudes estadísticas como las correlaciones cruzadas de las señales generadas.

El método que proponen Mengali y Micheli [115], emplea filtros lineales alimentados por procesos blancos no-Gausianos, lo que les permite obtener señales características espectrales conocidas y asignadas a momentos de cuarto orden. Para simular un caso tridimensional, se escogen tres procesos blancos no-Gausianos independientes $\mathrm{x}_{i}(t), i=1,2,3$, como entradas de un sistema lineal con una matriz de transferencia $\mathcal{H}(t)$. Esta matriz se escoge bajo la restricción de que las salidas $\mathrm{y}_{i}(t), i=1,2,3$ tengan una matriz espectral asignada. En el esquema siguiente se muestra el proceso de la generación de estas salidas. 


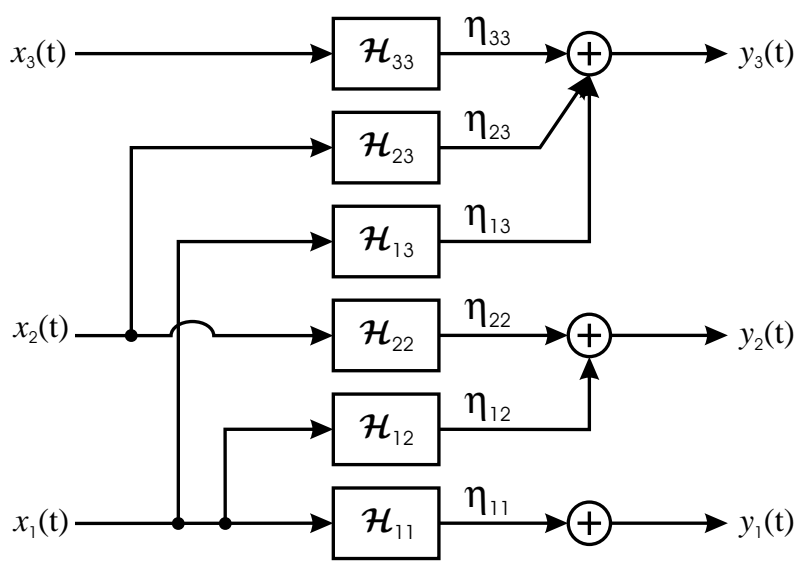

Figura 5.1: Esquema del proceso de generación de tres salidas

Podemos concluir diciendo que las señales generadas de esta forma pueden ser empleadas para representar componentes de velocidades promediadas en flujos turbulentos, y muestran una aplicación interesante en situaciones prácticas donde el modelo de señales Gausianas no es adecuado. En particular Mengali y Micheli [115] aplican este método a dos casos diferentes, por un lado simulan un flujo turbulento a la salida de un chorro y por otro simulan las componentes de velocidad en un campo atmosférico turbulento. Los resultados de estas aplicaciones demuestran que las señales no Gausianas con propiedades estadísticas dadas pueden ser simuladas con un alto grado de precisión.

En resumen, todos los estudios anteriores revelan la palpable necesidad de la existencia de un completo conjunto de aproximaciones teóricas eficientes avaladas por investigaciones experimentales y computacionales para un flujo definido, lo que permitiría una mayor comprensión de los complejos mecanismos de generación sonora, así como la posibilidad de realizar un control sobre estos fenómenos y no solamente el poder estimar aproximadamente el nivel sonoro en el campo lejano.

\subsection{Cálculos acústicos mediante métodos numéricos}

Para la predicción precisa del ruido generado por flujos turbulentos hemos de recordar que ésta involucra tres problemas: (i) el cálculo del campo de flujo turbulento subyacente, (ii) la predicción del ruido generado por tales flujos y (iii) la predicción de la propagación de las ondas acústicas en el medio inhomogeneo confinado por el flujo. 
En relación a todos los problemas planteados en el cálculo de la radiación acústica, las diferentes formulaciones de términos fuente y propagación sonora fueron analizadas en el capítulo anterior, y la forma de simulación numérica se ha abordado en la sección precedente, de modo que únicamente resta comentar la forma del cálculo directo final del sonido generado, el cual se basa en el desarrollo numérico de las teorías y analogías acústicas existentes empleando en algunos casos los resultados numéricos de los campos simulados.

El cálculo del flujo turbulento para geometrías realistas y números de Reynolds de interés práctico debe llevarse a cabo (Woodruff et al. [178]) a través de la solución directa de las ecuaciones de Navier-Stokes y Reynolds para un campo medio (RANS: Reynolds-Average Navier-Stokes equations), acopladas con los modelos de turbulencia, aunque éstos, como vimos en el apartado anterior, no son siempre fiables.

En relación al cálculo directo del sonido radiado, se comentarán a continuación algunos de los trabajos realizados por diferentes autores, así como los resultados conseguidos en ellos.

Woodruff et al. [178], examinan en un trabajo reciente el efecto de las representaciones alternativas del espectro de la turbulencia en los términos fuente de la analogía de Lighthill. En particular para el cálculo acústico hacen uso de la formulación de Lilley de la analogía acústica (ver apéndice A) y de las correcciones convectivas propuestas por Ffowcs Williams [109], introduciendo la separación de las correlaciones de velocidad en factores de dependencia temporal y de dependencia espacial. Los datos aerodinámicos requeridos para el cálculo acústico son calculados empleando el código de CFD ISAAC (Integrated Solution Algorithm for Arbitrary Configurations), que es un código de segundo orden de precisión de volúmenes finitos para resolver las ecuaciones completas de Navier-Stokes. Los términos convectivos son discretizados mediante un esquema upwind. En primer lugar se separan los términos de difusión y en segundo lugar se integran los sistemas de ecuaciones empleando una separación espacial y un esquema de factorización aproximada implícito. Los cálculos se llevan a cabo a través de la resolución de las ecuaciones de RANS, encontrándose la velocidad media del campo con la ayuda del modelo de turbulencia $\mathrm{k}-\varepsilon$ de dos ecuaciones. Los resultados obtenidos con este método para el caso de un chorro y comparados con los resultados experimentales manifiestan que en general este cálculo subestima el ruido a baja frecuencia. Por otro lado, mientras que los resultados experimentales muestran una marcada reducción de los picos del nivel de presión sonora (SPL) con la frecuencia cuando el ángulo polar aumenta, la predicción no verifica esta propiedad. 
Michalke [116], presenta los resultados de la aplicación de la ecuación diferencial inhomogenea derivada de la teoría de Neise para obtener la presión $p^{\prime}(x, t)$ generada por una presión externa dentro de una sonda del tipo 'slittube'. De esta forma se muestra, que si se emplea una sonda de este tipo para medir los modos que se propagan en un conducto, el espectro obtenido será probablemente menor que el real, ya que, las contribuciones de los modos acústicos de orden superior al de propagación de onda plana son sensiblemente subestimados.

Leung et al. [100], presentan un modelo simplificado del ruido generado por una pareja de vórtices con el objeto de modelar y reproducir las medidas del ruido generado por un chorro. Este modelo numérico del mutuo deslizamiento a través de anillos vorticales, tiene en cuenta los procesos de formación de las estructuras del chorro y sus subsiguientes movimientos de emparejamiento. La circulación de los anillos vorticales, tamaño del núcleo y radio, están relacionados en este modelo con la velocidad del chorro a través de fórmulas empíricas en función de diámetros característicos y números de Strouhal de las estructuras del flujo.

Con este modelo se calculó en primer lugar el movimiento de los anillos vorticales y posteriormente se evaluó el ruido radiado al campo lejano mediante la fórmula de Möhring [118]. Se encontró que este modelo conducía a la ley de la octava potencia (consistente con la teoría de Lighthill) para el ruido de chorro a bajos números de Mach. También se encontró que el sonido calculado se incrementaba linealmente con el diámetro de la tobera, lo cual es nuevamente consistente con las teorías existentes sobre el ruido de chorro para ángulos de emisión de $90^{\circ}$ y bajos números de Mach [100]. Por otro lado, el modelo presentado por Leung mostraba que las altas fluctuaciones de presión en el campo próximo no se correspondían con la radiación más importante en campo lejano. Leung et al. atribuyen esta differencia al hecho de que la presión medida en campo próximo está asociada al mivimiento del fluido en el chorro a la entrada de la región de excitación de dicho chorro. Y su magnitud está más directamente relacionada con el esfuerzo de las estructuras de flujo que con la potencia sonora producida por su interacción.

Lee y Koo [96] estudian la posible aplicación de una técnica de cálculo aeroacústico al caso de una pareja de vórtices giratorios para investigar la generación sonora por fuentes cuadripolares en flujos vorticales inestables. Esta técnica se basa en ecuaciones de Euler divididas en términos hidrodinámicos y términos de perturbación acústica. De este modo, se encontró que el sonido generado por fuentes cuadripolares de flujos vorticales inestables en la ausencia de la superficie de un cuerpo generador (fuentes monopolares o dipo- 
lares), podía ser calculado usando términos fuente debidos a las fluctuaciones de presión hidrodinámicas.

Howe es uno de los autores que mayor número de trabajos ha desarrollado dentro del campo del cálculo directo del ruido radiado por diferentes estructuras, en particular en el estudio de diferentes cuerpos, oscilaciones, etc., que inmersos en un fluido generan estructuras vorticales y por tanto fuentes sonoras. Dentro de sus últimas investigaciones, cabe mencionar la teoría lineal desarrollada para la interpretación de las oscilaciones automantenidas en capas de cizalla de altos números de Reynolds y chorros con números de Mach infinitesimales incidiendo sobre bordes y cavidades [78]. Oscilaciones que son generalmente fuentes de sonido de banda ancha, y se atribuyen en general a la formación de vórtices discretos cuya interacción con un borde o cavidad producen presiones impulsivas que conducen a la formación de nuevas vorticidades y completan un ciclo de retroalimentación. Este mecanismo de retroalimentación, conlleva una modificación de las longitudes características de singularidades como aperturas rectangulares en una pared y cavidades poco profundas, y por tanto, producen estados de radiación de diferente número de Strouhal característicos, valores que Howe predice detalladamente en este estudio.

Otro de los grandes campos de aplicación de la aeroacústica, es el del estudio del ruido generado por las capas de inestabilidad que rodean las alas de avión, campo que se sale del objetivo de nuestros estudios pero donde merecen mencionarse, por su relevancia, los trabajos de Howe [81,82].

Finalmente, no deben olvidarse las aportaciones realizadas al problema del calculo directo del ruido de flujo por autores como Goldstein y Leib; estos autores publicaron recientemente un método aproximado para el cálculo del ruido generado por un flujo turbulento dentro de un conducto seminfinito de sección transversal arbitraria [68]. Éste se basa en la ecuación de alta frecuencia derivada por Lilley, donde se simplifica el término fuente bajo la consideración de que la turbulencia en la dirección del flujo medio es axisimétrica. Uno de los puntos más interesantes de este trabajo es el cálculo que realizan del espectro radiado por una distribución de cuadripolos. 


\subsection{Estudio computacional del ruido de flujo gene- rado por silenciadores}

De la revisión bibliográfica realizada tanto en el capítulo anterior como en el presente, se deduce la escasa existencia de información e implementación de algoritmos acústicos para el estudio del ruido de flujo generado por fuentes internas de elementos como silenciadores y su posterior radiación al campo libre. Sin embargo, los estudios aeroacústicos existentes en la literatura sí que aportan importantes guías de actuación así como directrices a seguir a la hora de intentar implementar un cálculo aeroacústico de nuestro sistema. Siguiendo parte de estas líneas guías se desarrolla un método de cálculo del ruido de flujo generado por silenciadores, mediante cálculos similares a los efectuados por Woodruff, et al. [178], adoptando al igual que Bailly et al. [5] la idea de sintetizar un campo de velocidades fluctuantes en el interior del silenciador considerado, conocido en cada punto del espacio, que proporcione el término fuente necesario para obtener la presión acústica emitida al campo libre empleando las analogías acústicas presentadas en el capítulo anterior y en el apéndice A.

Como se ha comentado al comienzo de esta sección, para la predicción del ruido de flujo generado por flujos turbulentos se deben resolver o considerar tres fases del problema hasta llegar al cálculo final del sonido radiado que son, por orden:

1. El cálculo del campo del flujo turbulento.

2. La propagación de las ondas acústicas en el medio considerado.

3. La predicción del ruido generado por tales flujos, que se suele llevar a cabo a través del uso de una de las analogías acústicas mencionadas en el capítulo anterior.

La forma en que se ha resuelto cada una de estas fases de aproximación al cálculo final del sonido generado por un flujo estacionario en el interior de un silenciador serán explicadas detalladamente a continuación.

En una primera etapa, se simula el flujo turbulento estacionario presente en el interior del silenciador, con la finalidad de obtener los datos del flujo requeridos para el cálculo acústico. Sabemos que los flujos turbulentos están caracterizados por campos de velocidades fluctuantes que se mezclan con las cantidades transportadas como momento, energía, etc., y como consecuencia 
de ello las cantidades transportadas también fluctúan. Debido a que estas fluctuaciones pueden ser de muy pequeña escala y alta frecuencia supone un alto coste computacional el simular directamente estos flujos en cálculos prácticos. Por tanto en lugar de optar por una simulación numérica directa de este flujo (DNS) se escoge un conjunto de ecuaciones que puedan ser promediadas temporalmente o manipuladas de otra forma para eliminar las pequeñas escalas, resultando un conjunto de ecuaciones que computacionalmente son menos costosas de resolver. De este modo el cálculo del flujo turbulento se lleva a cabo a través del empleo de un código CFD comercial (FLUENT) que soluciona el sistema de ecuaciones de Navier-Stokes y Reynolds medias (RANS), acoplado con un modelo de turbulencia que sirve como cierre de este sistema. En este sistema de ecuaciones las ecuaciones de Navier-Stokes se descomponen en la componente media (en cada punto del espacio y el tiempo) y componentes fluctuantes. Para las componentes de velocidad esto se traduce en $u_{i}=\overline{u_{i}}+u_{i}^{\prime}$, donde $\overline{u_{i}}$ y $u_{i}^{\prime}$ son las componentes media e instantánea de la velocidad.

En el caso considerado se simula un flujo estacionario en el interior de un silenciador utilizando un algoritmo de precisión de primer orden en el código de volúmenes finitos (discretización mediante diferencias finitas) para resolver las ecuaciones completas de Navier-Stokes. Discretizando los términos convectivos de estas ecuaciones mediante un esquema upwind basado en el esquema de pressure-correction, y los términos de difusión mediante un esquema de diferencias centradas y de segundo orden de precisión.

A su vez, la aproximación escogida para linealizar y resolver las ecuaciones discretas RANS (Segregated, implícito), está basada en un algoritmo de acoplamiento de la presión-velocidad denominado SIMPLE (Semi-Implicit Method for Pressure-Linked Equations); este algoritmo emplea un procedimiento predictor-corrector para encontrar el campo de presión que da lugar a un campo de velocidad que satisfaga simultáneamente las ecuaciones de continuidad y momento [112]. La secuencia de operaciones que lleva a cabo es la siguiente:

1. Suponer un campo de presión $p^{*}$.

2. Evaluar la densidad $\rho^{*}$ a partir de la ley del gas.

3. Resolver las ecuaciones de momento para obtener los campos de velocidades $u_{1}^{*}, u_{2}^{*}$ usando las presiones y densidades supuestas $p^{*}$ y $\rho^{*}$.

4. Resolver la ecuación de corrección de la presión.

5. Corregir la presión a través de la igualdad $p^{\prime}=p-p^{*}$ 
6. Corregir las velocidades $u_{1}^{*}, u_{2}^{*}$ mediante las formulas de corrección $\left(u_{1}^{\prime}=\right.$ $u_{1}-u_{1}^{*}, u_{2}^{\prime}=u_{2}-u_{2}^{*}$ ) para obtener los campos de velocidad $u_{1}, u_{2}$ que satisfacen las ecuaciones de continuidad y momento.

7. Corregir la densidad a partir de la ecuación de estado del fluido considerado (en este caso aire).

8. Repetir desde el paso 1 , empleando la presión corregida $p$ con la nueva $p^{*}$, hasta que se consiga que el sistema converja a la solución real buscada.

De esta forma, en este cálculo de las ecuaciones de RANS se obtiene el campo de velocidad medio mediante la ayuda de un modelo de turbulencia $\mathrm{k}-\varepsilon$ estándar de dos ecuaciones. Modelo que emplea las hipótesis de Boussinesq para relacionar el tensor de esfuerzos de Reynolds $\rho u_{i}^{\prime} u_{j}^{\prime}$ con los gradientes de velocidad media $\partial u_{i} / \partial x_{i}$. La ventaja de esta aproximación es el relativamente bajo coste computacional asociado al cálculo de la viscosidad turbulenta, $\mu_{t}$, que se resuelve mediante el empleo de dos ecuaciones de transporte adicionales, una para la energía cinética turbulenta, k, y otra para la velocidad de disipación turbulenta, $\varepsilon$. La desventaja del uso de las hipótesis de Boussinesq es que se asume que $\mu_{t}$ es una cantidad escalar isótropa, lo que no es estrictamente cierto, sobre todo en el caso de flujos en los que la anisotropía de la turbulencia tiene un efecto dominante como es el caso de flujos con gran cantidad de remolinos. Sin embargo, este no es el caso del flujo estudiado, por lo que esta aproximación de la turbulencia se considera válida para el modelado del flujo estacionario en el interior del silenciador.

Una vez se ha obtenido el campo de velocidades y presiones en el interior del silenciador, nos enfrentamos a la tarea de generar un campo de velocidades fluctuantes que dé cuenta del movimiento tridimensional de este flujo turbulento en cada una de las celdas (volumen finito) del dominio considerado (silenciador). Este campo de velocidades se construye corrigiendo el campo de velocidades obtenido $u_{i}$ con la energía cinética turbulenta, k, magnitud que se considera que está íntimamente relacionada con la generación de turbulencia [67] y por tanto con las fluctuaciones de la misma. La definición que se adopta para esta magnitud, k, es la de una energía cinética promediada en masa $[6]$

$$
\mathrm{k}=\frac{1}{2} \overline{u_{i}^{\prime} u_{i}^{\prime}}
$$


De esta forma la magnitud de la velocidad de este nuevo campo de velocidad $u_{i}^{\prime \prime}$, vendrá dada por la siguiente expresión

$$
u_{i}^{\prime \prime}=u_{i} \cdot \sqrt{\frac{2 \cdot \mathrm{k}}{|\mathbf{u}|^{2}}}
$$

\subsubsection{Cálculo de la presión acústica radiada}

Conocidas las variables aeroacústicas básicas del flujo en el interior del silenciador a través de la simulación descrita anteriormente, queda propagarlas al punto de observación. Puede que este sea uno de los puntos más débiles del método de cálculo que proponemos, ya que se ha considerado propagación en campo libre debido a la simplicidad que presenta la función de Green necesaria para resolver la ecuación de ondas en este caso ${ }^{1}$. Aproximación que sólo es estrictamente válida para el caso en que la fuente se encuentre en el extremo de la cánula de salida y por tanto su propagación hasta el punto de observación, situado en un punto alejado de esta sección e inmerso en una atmósfera en reposo y libre de superficies reflectantes, coincida con la propagación en campo libre. El hecho de escoger esta forma de propagación se debe a la complejidad y en algunos casos desconocimiento de las funciones de Green en todas las regiones internas de la geometría considerada (tubos, cámaras, etc.), lo que hace difícil realizar una propagación adecuada de estas fuentes. Por ello, con la finalidad de reducir las imprecisiones que puede introducir este proceso de propagación del término fuente, ésta se realiza en dos fases como se esquematiza en la figura 5.2.

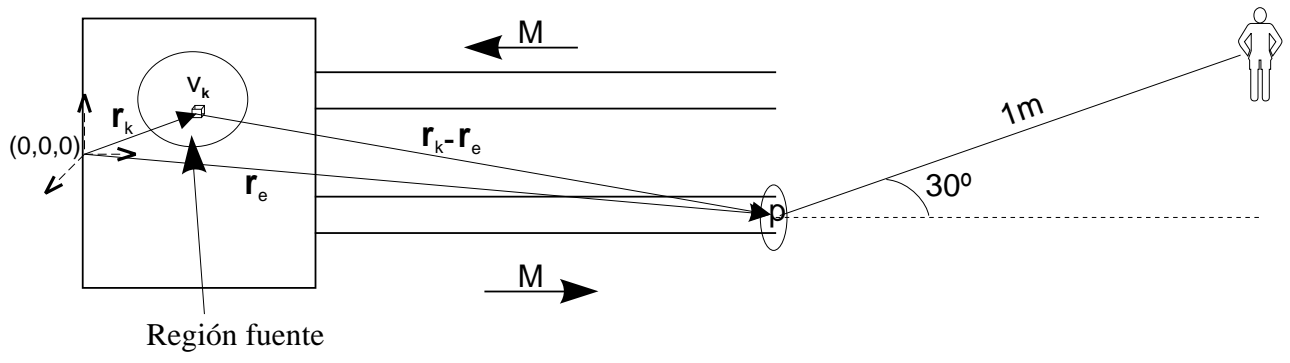

Figura 5.2: Esquema de los pasos seguidos para la propagación de una fuente interna de la geometría considerada, hasta el punto de observación en el campo libre.

\footnotetext{
${ }^{1}$ Expresión contenida en el apéndice B.
} 
Los dos pasos seguidos en la propagación de la fuente interna del silenciador hasta el punto de observación, situado a $1 \mathrm{~m}$ del extremo de salida de la cánula de escape y formando $30^{\circ}$ con la horizontal, se detallan a continuación:

1. En primer lugar los términos fuentes internos, $\mathbf{r}_{k}$, son propagados hasta el extremo del conducto, $\mathbf{r}_{e}$, es decir se realiza una especie de traslado espacial de la fuente hasta dicho punto como si el observador estuviera situado en él, haciendo uso de la expresión de la función de Green en campo libre. De esta forma se obtendrá una presión global, $p$, en el extremo de la cánula de salida, que será la que se propague al exterior en un segundo paso.

2. En este segundo y último paso se propaga la presión global obtenida en el extremo abierto de la cánula de salida al punto de observación emplazado a $1 \mathrm{~m} \mathrm{y} 30^{\circ}$ de esta sección, situación en la que es válido asumir una radiación isótropa al campo libre a través de la función de Green conocida.

Este punto de observación $\left(1 \mathrm{~m}, 30^{\circ}\right)$ se escoge con el objetivo de poder comparar estos resultados con los valores de ruido global obtenidos experimentalmente y mostrados en el capítulo anterior.

Una vez explicado el algoritmo de propagación del término fuente sólo resta conocer este término, $q$. Para ello y continuando con la aplicación de las analogías acústicas se obtiene la presión acústica resultante en un punto r y en un tiempo $t$ resolviendo la integral que resulta de la resolución de la ecuación de ondas $\frac{1}{a_{o^{2}}} \frac{\partial^{2} p}{\partial t^{2}}-\nabla^{2} p=q$, empleando la expresión de la función de Green de propagación en campo libre (como se ha comentado):

$$
p(\mathbf{r}, t)=\frac{1}{4 \pi} \cdot \int_{v} q \frac{d \mathbf{r}^{\prime}}{\left|\mathbf{r}-\mathbf{r}^{\prime}\right|}
$$

donde $\mathbf{r}^{\prime}$ representa la posición del término fuente, considerado como una fuente compacta, y por tanto puntual, vista desde el observador situado en el campo lejano (campo libre). El valor de $q$ se escogerá partiendo del término fuente desarrollado por Lighthill, $q=\frac{\partial^{2} T_{i j}}{\partial x_{i} \partial x_{j}}$, ya que es el más comúnmente aceptado y de donde parten el resto de teorías acústicas, además de que como sugiere Crighton [32] tiene una forma simple pero dotada de excelente robustez y capaz de revelar importantes rasgos del campo sonoro. En él, como se mencionó en el capítulo anterior, $T_{i j}=\rho u_{i} u_{j}+p_{i j}-a_{o}^{2} \rho \delta_{i j}$, representa el tensor de esfuerzos de Lighthill, donde: 
- $u_{i}$ son las componentes del campo de velocidades del fluido

- $p_{i j}=p \delta_{i j}-\sigma_{i j}$, es el tensor de esfuerzos compresivo y $\sigma_{i j}$ es el tensor de esfuerzos viscosos.

- $a_{o}$ es la velocidad del sonido del medio exterior

- $\rho$ y $p$ son la densidad y presión del medio

A modo de recordatorio de lo comentado en el capítulo anterior, digamos que el término del tensor de Lighthill, bajo ciertas consideraciones, se reduce a $T_{i j}=\rho_{o} u_{i} u_{j}$. Estas consideraciones son:

- Para flujos en los que la temperatura se desvía poco de la uniformidad, como es el caso considerado (flujo frío), las diferencias entre el campo de presión exacta $p_{i j}$ y su forma aproximada $a_{o}^{2} \rho \delta_{i j}$ son igualmente poco importantes, ya que:

1. Los números de Reynolds considerados $8 \cdot 10^{4}-3 \cdot 10^{5}$ son suficientemente elevados para asegurar que $\rho u_{i} u_{j} \gg \sigma_{i j}$.

2. Se puede asumir la evolución del fluido como isoentrópica (influencia de la temperatura despreciable), y por tanto: $p^{\prime} \cong a_{o}^{2} \rho^{\prime}$, anulándose así el segundo término de $p_{i j}$.

Dicho esto, el mecanismo principal de generación de sonido se encuentra recogido en el primer término del desarrollo de $T_{i j}$, y el término fuente puede ser escrito como:

$$
q=\frac{\partial^{2}\left(\rho_{o} \cdot u_{i} \cdot u_{j}\right)}{\partial x_{i} \partial x_{j}}
$$

con un error del orden del cuadrado del número de Mach, $M$, siendo $\rho_{o}$ la densidad a temperatura ambiente.

Una vez adoptada la expresión del término fuente (5.4) ésta se sustituye en la ecuación (5.3) y se desarrolla de forma explícita para calcular la presión emitida al campo libre debida a cualquier fenómeno de generación sonora asociado a esta fuente. De esta sustitución se deriva [102] la siguiente ecuación para la presión transmitida.

$$
p(\mathbf{r}, t)=\frac{1}{4 \pi} \frac{\partial^{2}}{\partial x_{i} \partial x_{j}} \int_{v}\left(\rho_{o} u_{i} u_{j}\right) \cdot \frac{d \mathbf{r}^{\prime}}{r}=\frac{\rho_{o}}{4 \pi} \int_{v} \frac{\partial^{2}}{\partial x_{i} \partial x_{j}}\left(\frac{u_{i} u_{j}}{r}\right) d \mathbf{r}^{\prime}
$$


donde $r=\left|\mathbf{r}-\mathbf{r}^{\prime}\right|$ es el modulo de la distancia desde el punto fuente $\mathbf{r}^{\prime} \mathrm{y}$ el punto de observación $\mathbf{r}$, y $\int_{v}$ representa la integración a todo el volumen ocupado por la fuente acústica.

Esta integral de volumen original se discretiza obteniendo así una forma aproximada de la presión acústica en términos de sumatorios para los elementos de volumen finito e infinitesimal, $\nu_{k}$, del cálculo computacional llevado a cabo, es decir, el volumen de las celdas del mallado del dominio considerado (el silenciador), expresándose la ecuación (5.5) como

$$
p(x, t)=\frac{\rho_{o}}{4 \pi} \cdot \sum_{k}^{N_{k}}\left(\sum_{i, j} \frac{\partial^{2}\left(\frac{u_{i} u_{j}}{r}\right)}{\partial x_{i} \partial x_{j}}\right) \nu_{k}
$$

Esta discretización de la integral volumétrica muestra la importancia que tiene el considerar un mallado adecuado para la obtención de buenos resultados a través de nuestro modelo, ya que el fenómeno a resolver se caracteriza por poseer en algunos casos pequeñas amplitudes de fluctuación sonora por lo que todos los defectos locales del modelo computacional tanto en el contorno como en el volumen, pueden tener una importante influencia en la evolución del flujo en el dominio espacial completo, pudiendo constituirse como una posible fuente de error [162] distorsionando el cálculo del flujo medio. De este modo el esquema de mallado escogido debe dar solución a un amplio rango de frecuencias sonoras empleando una malla con el mínimo tamaño de celda posible, el cual debe ser menor que la mínima longitud de onda sonora considerada [63]. Esta malla debe además mostrar una cierta homogeneidad en todo el dominio de cálculo para que no se produzcan grandes distorsiones en el cálculo del flujo.

Para simplificar la notación consideraremos la expresión del tensor de esfuerzos como: $T_{i j}=\rho_{o} u_{i} u j$, de manera que la expresión anterior podría escribirse como:

$$
p(\mathbf{x}, t)=\frac{1}{4 \pi} \sum_{k=1}^{N_{k}}\left[\sum_{i, j=1}^{3}\left(\frac{\partial^{2}}{\partial x_{i} \partial x_{j}}\left(\frac{1}{r} \cdot T_{i j}\right)\right)\right] \nu_{k}
$$

donde:

- $N_{k}$ es el número total de celdas pertenecientes a la región fuente.

- $T_{i j}=\rho_{o} \cdot u_{i} \cdot u_{j}$ representa el tensor de esfuerzos de Lighthill 
- $u_{i}$ son las componentes del campo de velocidades del flujo asociado a cada dirección y celda.

- $x_{i}$ son las coordenadas de dirección del vector $\mathbf{r}$ del punto de observación.

- $r=\left|\mathbf{r}-\mathbf{r}^{\prime}\right|$ donde $\mathbf{r}$ es el vector de posición del observador, y $\mathbf{r}^{\prime}$ es el vector de posición de la fuente, que en este caso se asumirá como la posición de cada una de las celdas pertenecientes a esa región fuente de coordenadas $\mathbf{r}_{k}=\left(y_{i}\right)_{i=1, . ., 3}$ como se observa en la gráfica 5.2.

Desarrollando el término entre corchetes en (5.7) se obtiene la siguiente expresión:

$$
\begin{aligned}
\sum_{i, j}^{3} \frac{\partial^{2}}{\partial x_{i} \partial x_{j}} \cdot\left(\frac{1}{r} \cdot T_{i j}\right)= & \frac{1}{r} \cdot \sum_{i}^{3} \sum_{j}^{3} \frac{\partial^{2} T_{i j}}{\partial x_{i} \partial x_{j}}- \\
& -\frac{1}{r^{3}} \cdot\left[\sum_{i}^{3} T_{i i}+2 \cdot \sum_{i}^{3}\left(x_{i}-y_{i}\right) \sum_{j}^{3} \frac{\partial T_{i j}}{\partial x_{j}}\right]+ \\
& +\frac{3}{r^{5}} \cdot \sum_{i}^{3}\left(x_{i}-y_{i}\right) \sum_{j}^{3}\left(x_{j}-y_{j}\right) T_{i j}
\end{aligned}
$$

En la expresión anterior (5.8), es posible observar la diferente contribución de cada uno de los términos asociados a diferentes decaimientos de las fuentes con la distancia. Esta expresión de la fuente corresponde a una suma de fuentes: monopolares, dipolares y cuadripolares, permitiendo analizar la diferente contribución de cada una de ellas en el campos próximo (a distancias próximas a la descarga del flujo) y lejano (campo libre), además de tener una visión global más adecuada de todos los posibles mecanismos de generación sonora que tienen lugar en el interior de los silenciadores. Los diferentes términos mostrados en la ecuación (5.8) son desarrollados a continuación:

$$
\begin{gathered}
\sum_{i}^{3} \sum_{j}^{3} \frac{\partial^{2} T_{i j}}{\partial x_{i} \partial x_{j}}=\rho_{o} \sum_{i}^{3} \sum_{j}^{3}\left(\frac{\partial^{2} u_{i}}{\partial x_{i} \partial x_{j}} u_{j}+\frac{\partial u_{i}}{\partial x_{j}} \frac{\partial u_{j}}{\partial x_{i}}+\frac{\partial u_{i}}{\partial x_{i}} \frac{\partial u_{j}}{\partial x_{j}}+u_{i} \frac{\partial^{2} u_{j}}{\partial x_{i} \partial x_{j}}\right) \\
\sum_{i}^{3} T_{i i}=\rho_{o} \sum_{i}^{3} u_{i}^{2} ; \quad \sum_{j}^{3} \frac{\partial T_{i j}}{\partial x_{j}}=\rho_{o} \sum_{j}^{3}\left(\frac{\partial u_{i}}{\partial x_{j}} u_{j}+u_{i} \frac{\partial u_{j}}{\partial x_{j}}\right)
\end{gathered}
$$




$$
\sum_{i}^{3}\left(x_{i}-y_{i}\right) \sum_{j}^{3}\left(x_{j}-y_{j}\right) T_{i j}=\rho_{o}\left(\sum_{i}^{3}\left(x_{i}-y_{i}\right) u_{i}\right)^{2}
$$

Para introducir un término fuente más general que incluya la contribución del sonido generado por la vorticidad, la expresión del término $\sum_{i, j}\left(\frac{\partial^{2} T_{i j}}{\partial x_{i} \partial x_{j}}\right)$ dada por la ecuación (5.9) se transforma empleando los resultados obtenidos por Powell-Howe (teoría descrita en profundidad en el apéndice A), quedando este término expresado como:

$$
\sum_{i, j} \frac{\partial^{2} T_{i j}}{\partial x_{i} \partial x_{j}}=\rho_{o} \sum_{i, j} \frac{\partial^{2} u_{i} u_{j}}{\partial x_{i} \partial x_{j}}=\rho_{o} \sum_{i, j} \nabla \mathcal{L}
$$

En esta analogía $\mathcal{L}=\boldsymbol{\Omega} \times \mathbf{u}$, siendo $\boldsymbol{\Omega}=\nabla \times \mathbf{u}$ el vector vorticidad del flujo, representa el vector de Lamb, que puede considerarse como la fuerza de Kutta-Joukowski sobre el elemento lineal del material, pudiendo aparecer como pares que se cancelan entre sí, si el momento resultante se conserva [32].

La importancia de considerar también el efecto de la vorticidad se debe a que en la formulación original de la analogía acústica de Lighthill $[102,103]$ las fuentes de sonido son descritas únicamente en términos de una transferencia de momento fluctuante, siendo obviadas las componentes solenoidales de la fluctuación de velocidad (vorticidad), que predominan en el desarrollo de las capas de corte, que son consideradas por Powell [139] a través del desarrollo de una formulación alternativa a la de Lighthill que describe las correspondientes fuentes acústicas en términos de fluctuaciones de vorticidad.

Una vez introducida la nueva expresión (5.12) para el primer término (5.9) de la ecuación general del término fuente (5.8), éste se desarrolla teniendo en cuenta la siguiente propiedad del vector $\nabla$ :

$$
\nabla(\mathbf{a} \times \mathbf{b})=\mathbf{b} \cdot(\nabla \times \mathbf{a})-\mathbf{a} \cdot(\nabla \times \mathbf{b})
$$

calculándose el término $\nabla \mathcal{L}=\nabla \cdot(\boldsymbol{\Omega} \times \mathbf{u})$ de la siguiente manera

$$
\begin{aligned}
\nabla \mathcal{L} & =\mathbf{u} \cdot(\nabla \times \boldsymbol{\Omega})-\boldsymbol{\Omega} \cdot(\nabla \times \mathbf{u})=\mathbf{u} \cdot(\nabla \times \boldsymbol{\Omega})-\boldsymbol{\Omega} \cdot \boldsymbol{\Omega}= \\
& =u_{x}\left(\frac{\partial \Omega_{z}}{\partial \mathrm{y}}-\frac{\partial \Omega_{y}}{\partial \mathrm{z}}\right)-u_{y}\left(\frac{\partial \Omega_{z}}{\partial \mathrm{x}}-\frac{\partial \Omega_{x}}{\partial \mathrm{z}}\right)+u_{z}\left(\frac{\partial \Omega_{y}}{\partial \mathrm{x}}-\frac{\partial \Omega_{x}}{\partial \mathrm{y}}\right)-|\boldsymbol{\Omega}|^{2}
\end{aligned}
$$

donde (x, y, z) representan las componentes de un sistema de referencia cartesiano y $\left(\Omega_{x}, \Omega_{y}, \Omega_{z}\right)$ son las coordenadas del vector vorticidad $\boldsymbol{\Omega}$ en este sistema de referencia, componentes que pueden obtenerse en función de las 
componentes del campo de velocidades del flujo $\mathbf{u}=\left(u_{i}\right)_{i=x, y, z}$ a partir de la definición de la vorticidad $(\boldsymbol{\Omega}=\nabla \times \mathbf{u})$ :

$$
\Omega_{x}=\frac{\partial u_{z}}{\partial \mathrm{y}}-\frac{\partial u_{y}}{\partial \mathrm{z}} ; \quad \Omega_{y}=\frac{\partial u_{x}}{\partial \mathrm{z}}-\frac{\partial u_{z}}{\partial \mathrm{x}} ; \quad \Omega_{z}=\frac{\partial u_{y}}{\partial \mathrm{x}}-\frac{\partial u_{x}}{\partial \mathrm{y}}
$$

Una vez conocida la expresión del término fuente (5.8) para obtener la presión acústica emitida en el punto de observación se empleará el campo de velocidades construido $u_{i}^{\prime \prime}(5.2)$, sustituyendo su expresión en la ecuación del término fuente (5.8). A partir de este término fuente se calcula la presión en un punto exterior a través del algoritmo de propagación mencionado anteriormente.

\subsubsection{Estimación de la intensidad sonora global transmitida al campo libre}

Una vez conocido el valor global de la presión acústica emitida al campo libre, $p$, es posible calcular la intensidad sonora generada en ese punto de observación a través de la relación entre presión e intensidad $I_{r}$ en campo libre:

$$
I_{r}=\frac{p^{2}}{\rho_{o} \cdot a_{o}}
$$

De cuya expresión se deduce que la dificultad en la estimación de la intensidad radiada radica en el calculo de la presión acústica radiada en el campo libre, el cual como se ha mencionado se realiza en dos pasos sucesivos. En un primer tramo se calcula la presión en el extremo del conducto empleando la ecuación (5.7) sustituyendo en ella el término fuente descrito (5.8) y considerando que el punto de observación es el extremo abierto. Conocida la presión total en este punto, para propagarla al exterior se tiene en cuenta la conservación de energía acústica asumiendo radiación isótropa de forma que toda la energía que fluya a través de la sección de salida será transformada en energía acústica en el punto de observación en el campo libre. Obteniendo finalmente la intensidad sonora en el campo libre corrigiendo la expresión (5.7) con la relación de áreas

$$
I_{r}(r)=\left(\frac{a}{r}\right)^{2} \frac{p^{2}}{\rho_{o} \cdot a_{o}}
$$

donde $p$ será el valor de la presión en el extremo del conducto, $r$ la distancia desde el punto de observación al extremo del conducto y a el radio de la cánula de salida. 
Como último comentario cabe resaltar que todas las teorías acústicas utilizadas (Lighthill, Powell) son válidas para el caso de fuentes compactas por lo que es necesario disponer de una adecuada descripción espacial de la fuente que permita acotarla. Es sabido que en el caso de la emisión por la descarga de un chorro esta fuente se localiza en un punto cercano a la sección de salida de la cánula de escape, en la dirección del flujo saliente [92]; sin embargo, no hay ninguna información sobre cuál es la localización de las fuentes en el interior de los silenciadores, a pesar de que los resultados mostrados en el capítulo anterior permiten aventurarlo, pareciendo lógico pensar que estas fuentes se situarán en zonas donde se observen valores máximos de la energía cinética turbulenta; ya que según afirman Bailly et al. [4], la producción de energía cinética turbulenta, k, para el caso de un chorro libre está directamente asociada con los gradientes de velocidad, que alcanzan valores máximos en las capas de mezcla y son despreciables en el cono de silencio. Por tanto los gráficos de las líneas de contorno de las distribuciones espaciales de $\mathrm{k}$ permiten establecer geométricamente qué zonas de la configuración estudiada se constituyen como regiones fuente más importantes, que serán las zonas asociadas a los máximos de esta magnitud [12].

De este modo se estudiaron diferentes limitaciones geométricas de la fuente en relación a los máximos de energía cinética turbulenta presentados en las simulaciones de las diferentes geometrías, analizando qué ventajas y grado de aproximación a la realidad ofrecen los resultados derivados de cada una de ellas. En este estudio, se empleó la misma geometría simple (cámara de expansión reversa) estudiada en capítulos anteriores, a fin de homogeneizar los resultados derivados en todos los capítulos de la presente tesis doctoral.

\subsection{Resultados de la aplicación a una geometría sim- ple}

En este último apartado se analiza la potencia del método de cálculo propuesto mediante su aplicación a la geometría simple estudiada en los capítulos anteriores, cámara de expansión reversa, evaluando diferentes definiciones geométricas de las regiones fuente por comparación con los escalamientos y tendencias observados experimentalmente. Estas regiones fuente se definen, como se indicó en la sección anterior a través del análisis de la energía cinética turbulenta generada en el interior de estas geometrías de modo similar a como lo realizan Béchara et al. [12].

La primera localización escogida para la región fuente es una región próxima a la entrada de la cánula de salida, basándonos en dos criterios: (i) por 
un lado debido a que la fuente de emisión externa está situada supuestamente [92] cerca de la boca de escape, en la dirección del flujo, parece lógico pensar que ésta esté inducida especialmente por la fuente situada a la entrada de esta cánula; (ii) en segundo lugar, las diversas geometrías modeladas muestran máximos de energía cinética turbulenta importantes en estas regiones como se puede comprobar en la figura 5.3.

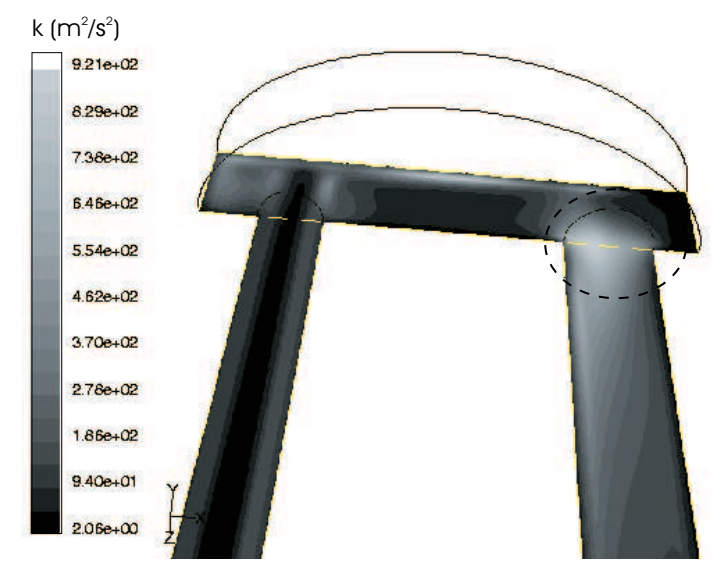

Figura 5.3: Energía cinética turbulenta simulada en una cámara de expansión reversa para una velocidad media del flujo de $70 \mathrm{~m} / \mathrm{s}$.

De este estudio se derivan dos tipos de resultados, unos referidos a las tendencias del ruido de flujo emitido y otros a los escalamientos con la velocidad. En primer lugar mostraremos las gráficas de tendencias obtenidas para estas geometrías mediante el procedimiento de cálculo propuesto, comparándolas con las obtenidas a través de las medidas de intensidad realizadas en una cámara semianecoica. Al igual que en el capítulo anterior, este análisis se realiza distinguiendo las cámaras reversas modeladas (las mismas que medidas) por conjuntos de geometrías del mismo diámetro de salida.

El análisis comparativo entre los resultados de ruido global obtenidos a partir de las medidas de intensidad (por integración de las mismas para todas las frecuencias), y las obtenidas mediante el cálculo descrito se presentan en la figura 5.4. En ella se puede observar que la reproducción de las tendencias medidas (gráficos de la izquierda) mediante el método de cálculo descrito no es exacta en ninguno de los tres casos, aunque sí algo aproximada, especialmente para las geometrías de $d_{o}=30 \mathrm{~mm}$ en que su comportamiento es más ajustado al observado experimentalmente. Lo mismo puede decirse de la comparación de las tendencias relativas entre estos tres conjuntos de geometrías presentados en la figura 5.5. 

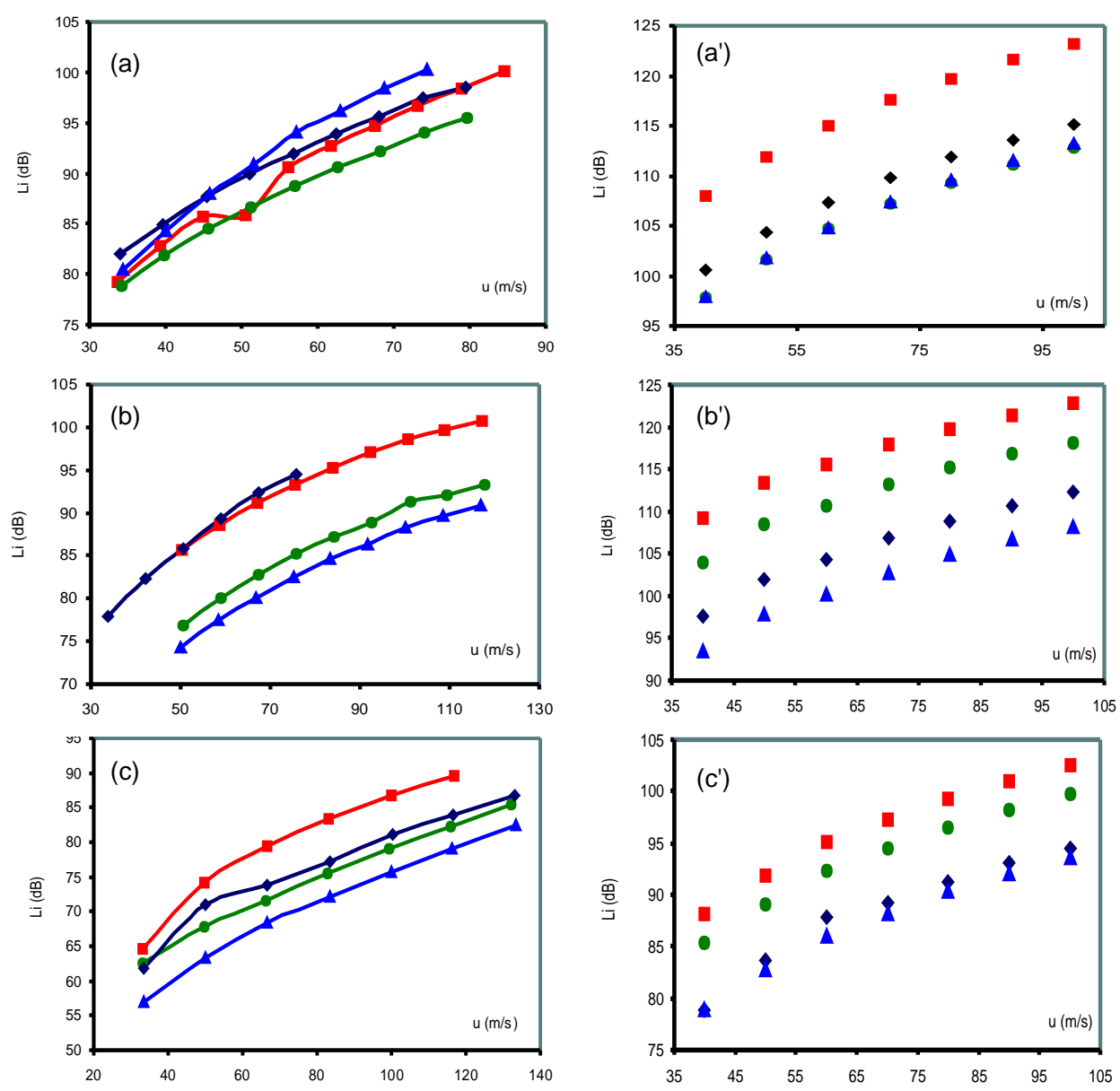

Figura 5.4: Análisis comparativo de las tendencias de las medidas de intensidad para las geometrías de: (a) $d_{o}=51 \mathrm{~mm},(b) d_{o}=42 \mathrm{~mm}$, (c) $d_{o}=30 \mathrm{~mm}$, y las reproducidas mediante el cálculo realizado: ( $\left.a^{\prime}\right) d_{o}=51 \mathrm{~mm},\left(b^{\prime}\right) d_{o}=42 \mathrm{~mm},\left(c^{\prime}\right) d_{o}=30 \mathrm{~mm}$. Donde: (曰) $L_{c}=25 \mathrm{~mm},(\bullet) L_{c}=50 \mathrm{~mm},(\bullet) L_{c}=100 \mathrm{~mm},(\boldsymbol{\Delta}) L_{c}=150 \mathrm{~mm}$. 

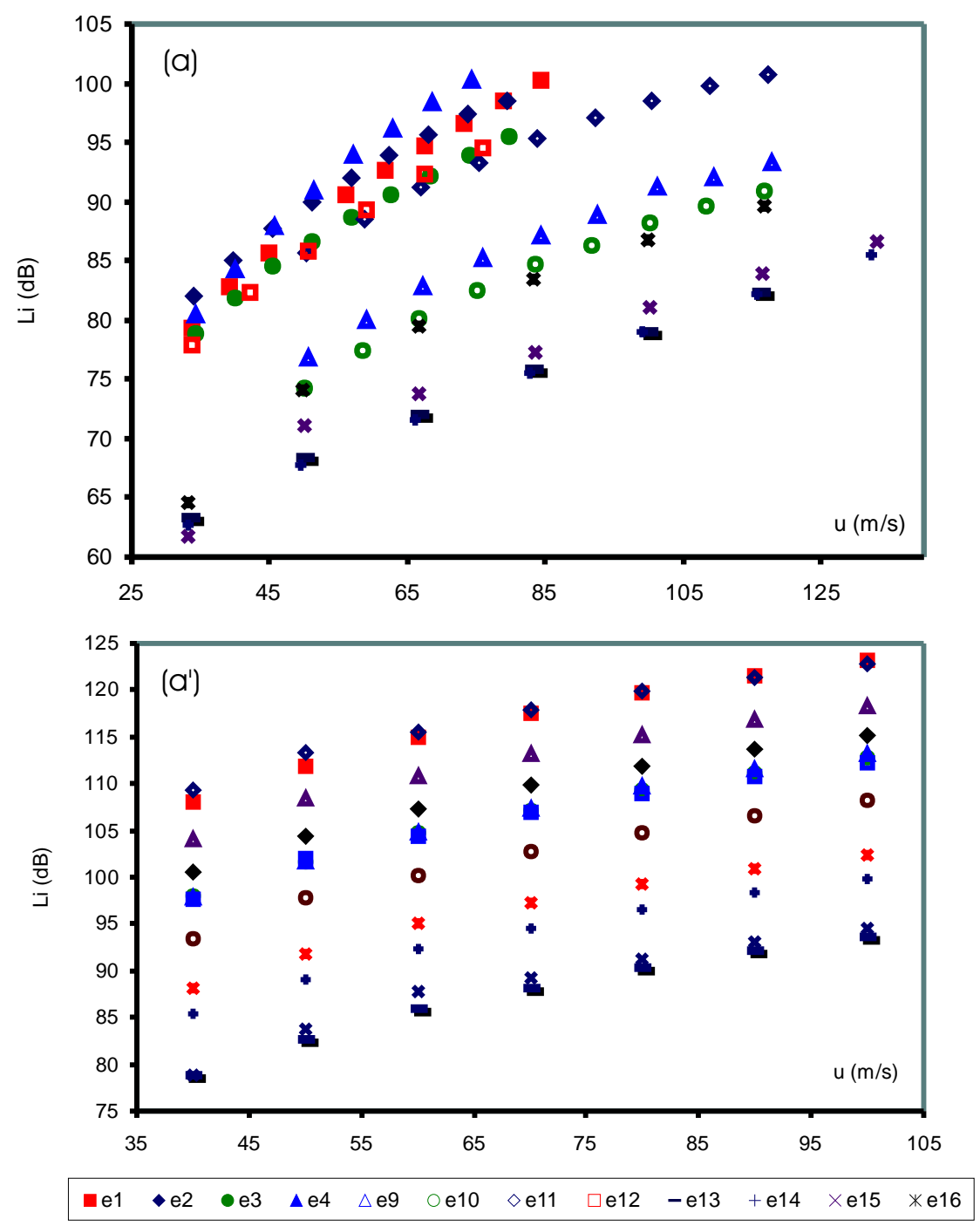

Figura 5.5: Análisis comparativo de las tendencias de la intensidad global entre todas las geometrías estudiadas: (a) medidas, y ( $\left.a^{\prime}\right)$ calculadas. 
La no correcta reproducción de las tendencias mostradas en las figuras anteriores, puede atribuirse a que la consideración de que la fuente interna se encuentra emplazada a la entrada de la cánula de salida no es suficiente, ya que como vimos en el capítulo anterior y puede comprobarse en la figura 5.6 , en el interior del silenciador analizado existen otras fuentes igualmente importantes. Con el fin de asegurar que el defecto de la escasa reproducción de tendencias se debe a no haber considerado todas las fuentes internas, y no a los problemas del modelo de cálculo propuesto, se analizan las tendencias reproducidas cuando se considera que la región fuente la constituye toda la cámara más la entrada de la cánula de salida. Consideración que dará idea de la posible influencia de otras fuentes internas sobre el ruido finalmente emitido a pesar de que no reproduzca una fuente exactamente compacta.
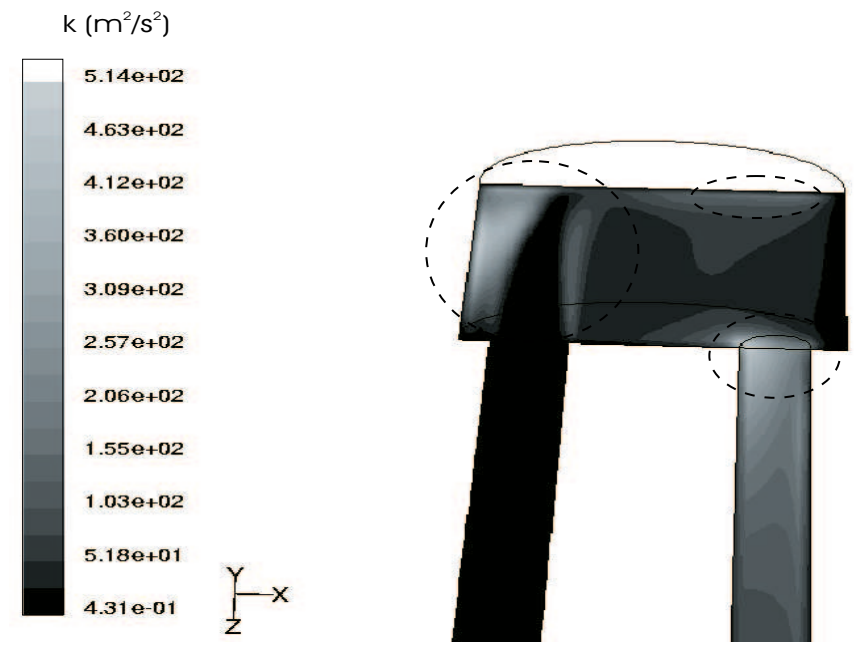

Figura 5.6: Energía cinética turbulenta simulada en una cámara de expansión reversa para una velocidad media del flujo de $70 \mathrm{~m} / \mathrm{s}$.

Las tendencias reproducidas con el cálculo de la intensidad considerando toda la cámara como región fuente, produce unos resultados mucho más ajustados a los observados experimentalmente, como se puede comprobar en la figura 5.7, apreciándose incluso un excelente acuerdo para las geometrías de

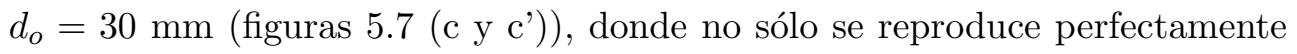
la tendencia esperable sino también las diferencias de niveles obtenidas en las medidas experimentales. Esto permite asegurar que nuestro modelo de cálculo es adecuado y válido para reproducir la evolución del ruido de flujo radiado por las diferentes geometrías. Con la finalidad de estudiar si el método es 
potente a la hora de establecer las tendencias relativas entre todo el conjunto de geometrías analizadas, en la figura 5.8 se recoge esta comparativa. En este caso se reproduce una evolución de la intensidad global muy pareja con la observada experimentalmente, efecto que no fue obtenido en el caso precedente (región fuente a la entrada de la cánula de salida).
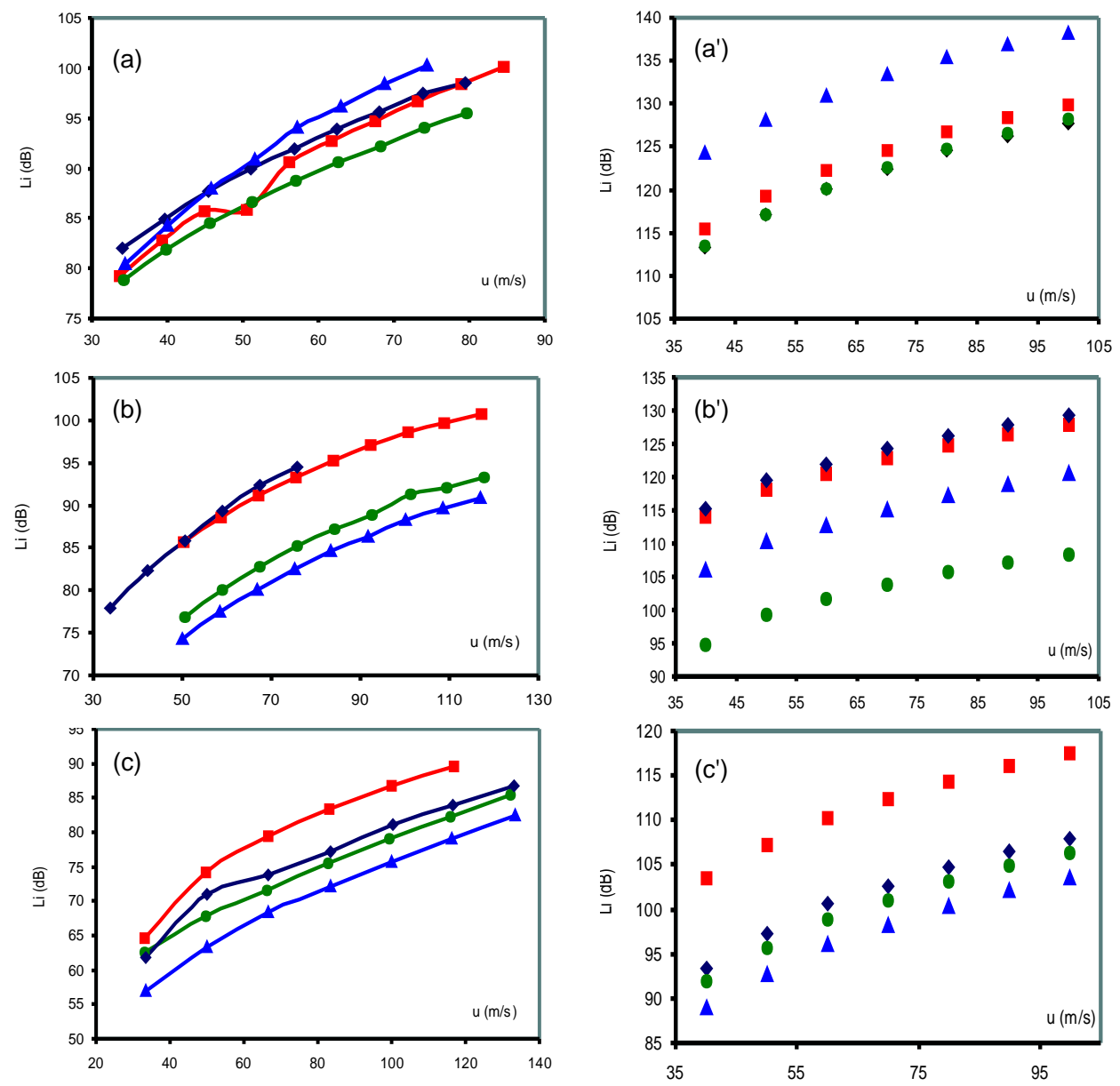

Figura 5.7: Análisis comparativo de las tendencias de las medidas de intensidad para las geometrías de: (a) $d_{o}=51 \mathrm{~mm},(b) d_{o}=42 \mathrm{~mm},(c) d_{o}=30 \mathrm{~mm}$, y las reproducidas mediante el cálculo realizado: ( $\left.a^{\prime}\right) d_{o}=51 \mathrm{~mm},\left(b^{\prime}\right) d_{o}=42 \mathrm{~mm},\left(c^{\prime}\right) d_{o}=30 \mathrm{~mm}$. Donde: (田) $L_{c}=25 \mathrm{~mm},(\bullet) L_{c}=50 \mathrm{~mm},(\bullet) L_{c}=100 \mathrm{~mm},(\boldsymbol{\Delta}) L_{c}=150 \mathrm{~mm}$. 


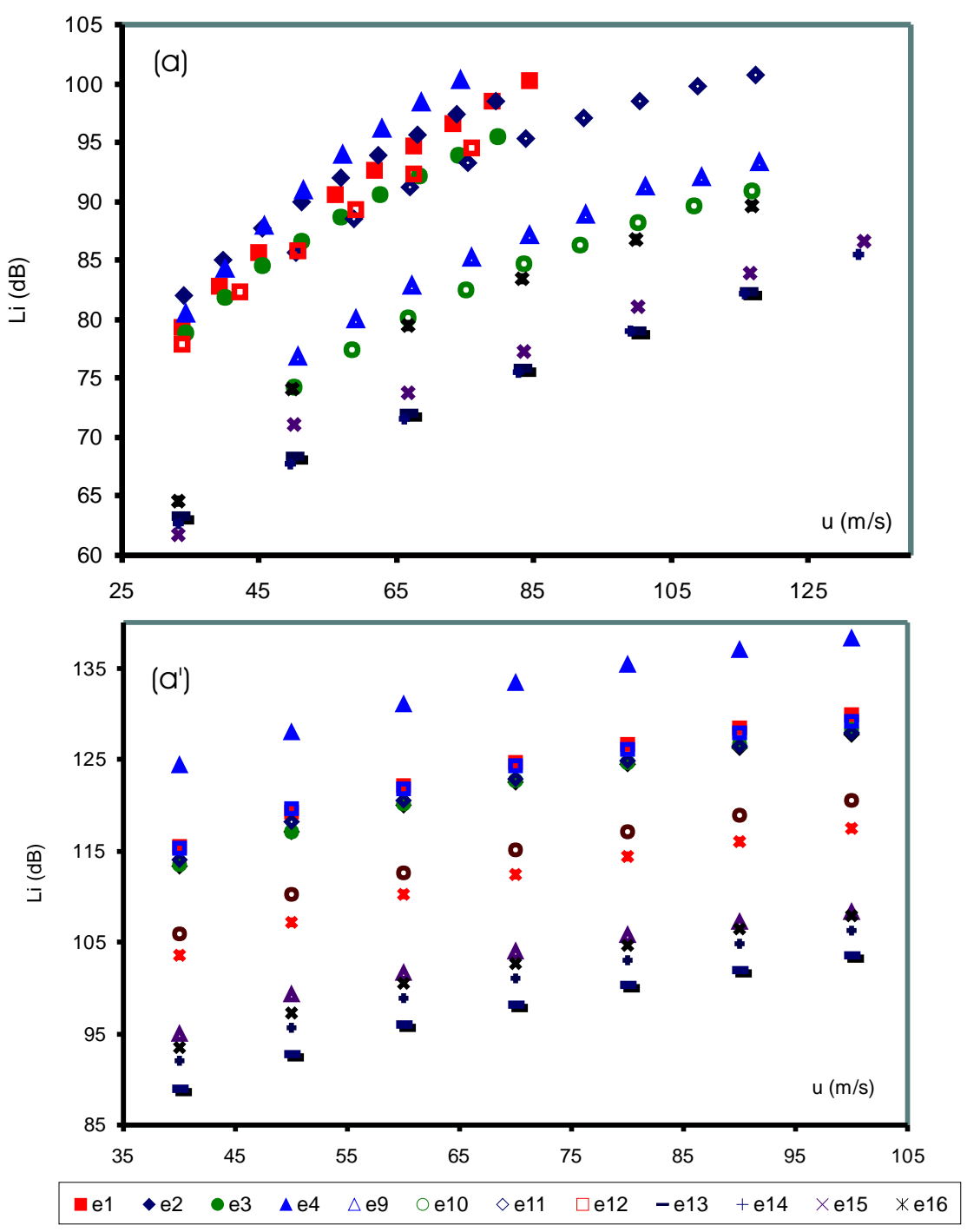

Figura 5.8: Análisis comparativo de las tendencias de la intensidad global entre todas las geometrías estudiadas: (a) medidas, y ( $\left.a^{\prime}\right)$ calculadas. 
Finalmente, a fin de verificar el efecto de la compacidad de la fuente considerada y establecer qué fuentes de las internas son más relevantes en las geometrías analizadas, se realiza un estudio análogo a los anteriores pero considerando únicamente la fuente situada a la salida de la cánula de entrada (originada por el fenómeno de separación del flujo que tiene lugar en esa sección), y la fuente a la entrada de la cánula de salida (debida a los efectos de la vorticidad generada por este cambio de sección). Ya que, como revelan los contornos de energía cinética turbulenta representados en la figura 5.6, ambas fuentes pueden considerarse como las de mayor intensidad. Los resultados de este estudio se presentan en las figuras 5.9 y 5.10 donde se representan la comparación de las evoluciones obtenidas de forma independiente para cada grupo de geometrías, y de forma global para todas las geometrías estudiadas, respectivamente. De ellas se extrae en primer lugar, que el hecho de considerar estas dos regiones del silenciador como fuentes principales proporciona unos resultados que muestran un acuerdo excelente para las geometrías de $d_{o}=42 \mathrm{~mm}$, tanto en tendencias de evolución como en diferencias de niveles observados, acuerdo no tan bueno en los otros dos casos, aunque sí bastante aproximado. Esto se debe a que para las geometrías de $d_{o}=42 \mathrm{~mm}$, estas regiones fuentes sí que parecen constituir las más relevantes del sistema, como se puede comprobar en la figura 5.11, y el hecho de haber acotado más las fuentes generadoras del sonido emitido permite que la hipótesis de fuente compacta se aproxime más a la realidad, y que por tanto nuestro método de cálculo sea más válido.

Por otro lado, para las geometrías de $d_{o}=51$ y $30 \mathrm{~mm}$ las fuentes principales de generación sonora no están tan claramente situadas en estas posiciones como se observa en la figura 5.11, lo que ratifica los mejores resultados obtenidos en estos casos al considerar como fuente toda la cámara (ver figura 5.7) a pesar de que la hipótesis de compacidad no sea del todo satisfecha con ese modelo.

Del mismo modo las evoluciones entre los diferentes grupos de geometrías obtenidos ahora (figura 5.10) aunque buenos, no son tan buenos como los reproducidos en el caso anterior (región fuente asociada a toda la cámara, figura 5.8), debido a los peores resultados obtenidos en este último caso para las geometrías de mayor y menor diámetro de la cánula de salida.

Siguiendo con el estudio, se podrían incluir otras regiones fuente, como la que se encuentra en la pared de salida de la cámara, sin embargo éstas parecen ser sensiblemente menos importantes por lo que no han sido analizadas. 

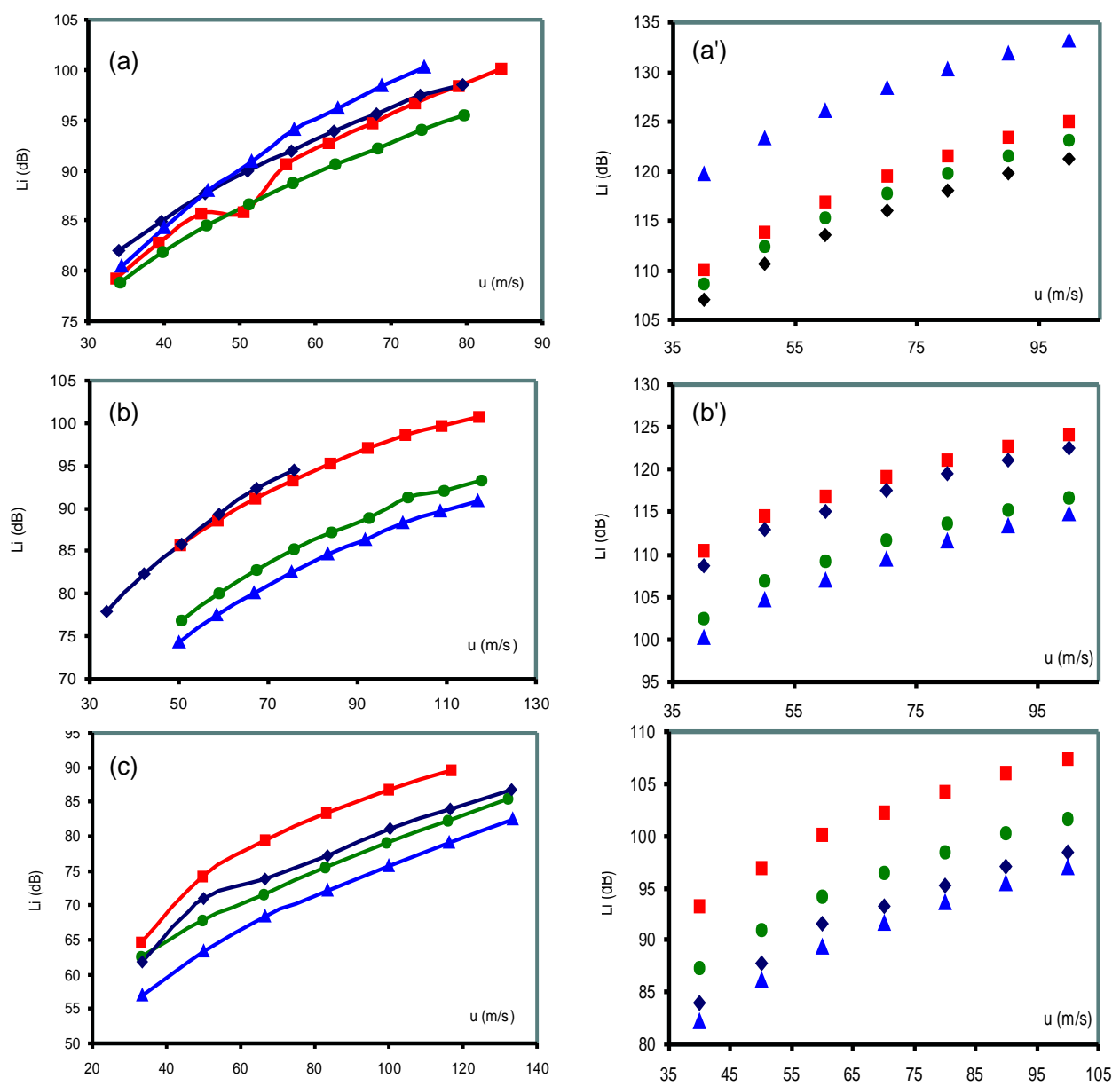

Figura 5.9: Análisis comparativo de las tendencias de las medidas de intensidad para las geometrías de: (a) $d_{o}=51 \mathrm{~mm}$, (b) $d_{o}=42 \mathrm{~mm}$, (c) $d_{o}=30 \mathrm{~mm}$, y las reproducidas mediante el cálculo realizado: ( $\left.a^{\prime}\right) d_{o}=51 \mathrm{~mm},\left(b^{\prime}\right) d_{o}=42 \mathrm{~mm},\left(c^{\prime}\right) d_{o}=30 \mathrm{~mm}$. Donde: (田) $L_{c}=25 \mathrm{~mm},(\bullet) L_{c}=50 \mathrm{~mm},(\bullet) L_{c}=100 \mathrm{~mm},(\boldsymbol{\Delta}) L_{c}=150 \mathrm{~mm}$. 

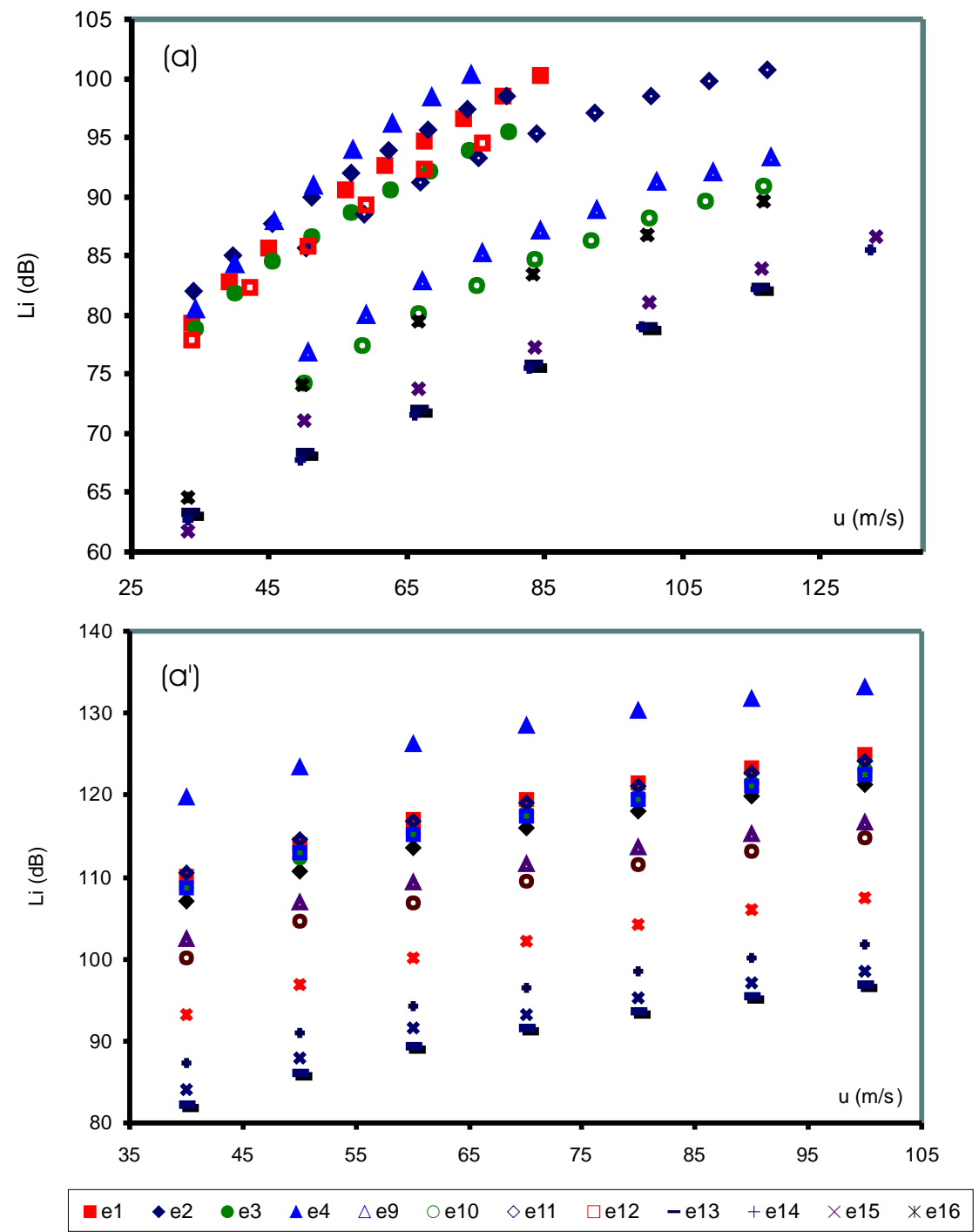

Figura 5.10: Análisis comparativo de las tendencias de la intensidad global entre todas las geometrías estudiadas: (a) medidas, y (a') calculadas. 


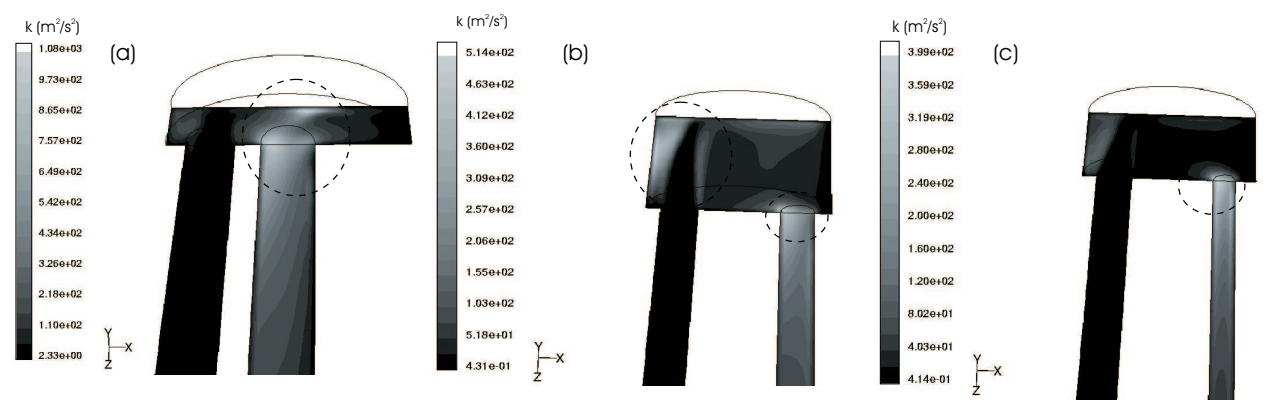

Figura 5.11: Distribución espacial de la energía cinética turbulenta simulada para diversas geometrías de una cámara de expansión reversa, (a) $d_{o}=51 \mathrm{~mm}$, (b) $d_{o}=$ $42 \mathrm{~mm}$ y (c) $d_{o}=30 \mathrm{~mm}$, para una velocidad media del flujo de $70 \mathrm{~m} / \mathrm{s}$.

Por último, del análisis realizado se concluye lo siguiente:

- Es necesario conocer y considerar con exactitud la posición de las fuentes internas del silenciador para obtener buenos resultados a través del procedimiento propuesto.

- En el caso de no ser conocidas con exactitud las principales fuentes internas, se obtienen resultados aceptables al considerar todas las posibles fuentes presentes en el silenciador (integración a toda la cámara), a pesar de que la hipótesis de compacidad de la fuente no sea satisfecha.

- El método geométrico de acotación de las fuentes asociado a los máximos de las distribuciones espaciales de energía cinética en el interior del silenciador es suficientemente potente.

- Este método complementa las medidas realizadas, al permitir identificar las principales fuentes de generación sonora, aportando mayor información sobre los fenómenos físicos asociados a la generación de ruido de flujo.

Una vez puesta de manifiesto la potencia del método para extraer las tendencias de evolución del ruido de flujo emitido por diferentes geometrías, se analiza la posibilidad de extrapolar su uso para la determinación de leyes de escalado, centrándonos únicamente en la posibilidad de reproducir el comportamiento monopolar, dipolar o cuadripolar de la fuente a partir del escalamiento del ruido emitido con la velocidad del flujo $(U)$ suponiendo que 
éste (el ruido global emitido $L_{i}$ ) muestra una ley de escalado de la siguiente forma:

$$
L_{i}(d B)=a+b \cdot(10 \cdot \log U)
$$

Recordamos que en el capítulo anterior se observó que las medidas de ruido radiado por estas cámaras mostraban comportamientos próximos a la monopolaridad en la mayor parte de los casos ( $b$ del orden de 4$)$ y en algunos otros tendían a comportamientos intermedios entre monopolar y dipolar $(b$ $=6$ ). A modo de resumen los diferentes valores de $a$ y $b$ obtenidos para los diferentes estudios realizados sobre las regiones fuentes se recogen en la figura 5.12, comparándolos con los derivados a través de las medidas de intensimetría en conductos. Técnica que fue validada en el capítulo anterior y que resulta ser más adecuada para comparar con los resultados derivados del método de cálculo propuesto, ya que en ambos casos sólo se contiene información de la fuente interna.

Del análisis de estas leyes de escalado, esto es, de los datos contenidos en la tabla de la figura 5.12, se derivan las siguientes conclusiones:

- Los cálculos realizados mediante el método de cálculo descrito en este capítulo (simulación computacional) muestran un comportamiento próximo a la monopolaridad en todos los casos $(b \sim 4)$, pero presentan en todos los casos valores inferiores a los obtenidos experimentalmente. De entre todas las consideraciones realizadas sobre la región fuente, la primera definición (región fuente considerada a la entrada de la cánula de salida) es la que proporciona valores más altos de este coeficiente, $b$, y por lo tanto más aproximados a la situación experimental. Esto se debe a que los errores cometidos al considerar propagación de la fuente en espacio libre son menos acusados que en las otras situaciones consideradas, además de ser la fuente más compacta de todas las consideradas. Ambas situaciones son las apropiadas para las leyes de escalado [52] (ecuación (5.17)) como se comentó en el capítulo anterior.

- Para la evolución de los exponentes de la velocidad (b) para las diferentes geometrías los mejores comportamientos se reproducen para la consideración de fuentes más compactas: regiones fuente definidas a la entrada de la cánula de salida ( $1^{a}$ región) o añadiendo a ésta la salida de la cánula de entrada ( $3^{a}$ región). Lo que parece estar de acuerdo con el hecho de que las leyes de escalado sólo indican la naturaleza de la fuente a la salida del sistema, siendo válidas únicamente para la emisión en campo libre de fuentes compactas [52]. 
- En la evolución de los términos constantes de la ley de escalado (a) se obtienen diferentes resultados, los cuales además entran en controversia en algunas ocasiones. Sin embargo, en general se puede decir que la $1^{a}$ y $2^{a}$ definición geométrica de la región fuente muestra buenos resultados para las geometrías de $d_{o}=30 \mathrm{~mm}$, lo cual revela que para obtener un buen escalado es importante considerar fuentes compactas y situaciones próximas a la emisión en campo libre, así como el haber considerado las fuentes principales de generación sonora en el interior del silenciador. Hecho que se corrobora en el mejor acuerdo obtenido para las geometrías de $d_{o}=42 \mathrm{~mm}$ para la situación intermedia entre ambos factores (fuentes más importantes y más compactas), esto es, la $3^{a}$ región fuente (entrada de la cánula de salida y salida de la cánula de entrada).

De todo el estudio realizado sobre la aplicación del modelo de cálculo propuesto a partir de la simulación computacional del campo de velocidades en el interior del silenciador, se puede concluir que es una metodología potente en relación al conocimiento de los principales mecanismos de generación sonora en el interior del silenciador, proporcionando a nivel cuantitativo buenos resultados en cuanto a la evolución del sonido total emitido al campo libre al realizarse cambios en la geometría en cuestión, si las regiones fuente principales son conocidas con exactitud. Podemos afirmar, además, que la buena reproducción de las tendencias ayuda a identificar las fuentes sonoras principales y por tanto, el conocimiento exacto de las mismas deja de ser una limitación al problema.

En relación a las leyes de escalado no se reproducen con exactitud las tendencias ni exponentes obtenidos, aunque sí que proporcionan una información aproximada del comportamiento real de la fuente en el campo libre. En este sentido, se piensa que mejores resultados se obtendrán si se mejora la propagación introduciendo las funciones de Green apropiadas para la propagación en el interior del silenciador, y si, por otro lado, se realizan simulaciones que contengan también la emisión del flujo a la atmósfera en reposo. Factores que introducirán previsiblemente mejoras al cálculo actual pero que en contrapartida supondrán un coste computacional superior. 


\begin{tabular}{|c|c|c|c|c|c|c|c|c|}
\hline \multirow{2}{*}{ Esquemas } & \multicolumn{2}{|c|}{ Medidas } & \multicolumn{2}{|c|}{$\begin{array}{c}\text { Región fuente } \\
1^{\mathrm{a}}\end{array}$} & \multicolumn{2}{|c|}{$\begin{array}{c}\text { Región fuente } \\
2^{\mathrm{a}}\end{array}$} & \multicolumn{2}{|c|}{$\begin{array}{c}\text { Región fuente } \\
3^{\mathrm{a}}\end{array}$} \\
\hline & $a$ & $b$ & $a$ & $b$ & $a$ & $b$ & $a$ & $b$ \\
\hline \multicolumn{9}{|c|}{$d=51 \mathrm{~mm}$} \\
\hline $\begin{array}{l}L_{c}=25 \mathrm{~mm}, d_{i}=51 \mathrm{~mm} \\
d_{c}=146 \mathrm{~mm}\end{array}$ & 41.3 & 5.38 & 47.1 & 3.82 & 57.7 & 3.62 & 50.3 & 3.74 \\
\hline $\begin{aligned} L_{c} & =50 \mathrm{~mm}, d_{i}=47 \mathrm{~mm} \\
d_{c} & =73 \mathrm{~mm}\end{aligned}$ & 53.2 & 4.68 & 42.02 & 3.67 & 55.1 & 3.65 & 49.5 & 3.60 \\
\hline $\begin{array}{l}L_{c}=100 \mathrm{~mm}, d_{i}=42 \mathrm{~mm} \\
d_{c}=146 \mathrm{~mm}\end{array}$ & 16.8 & 4.84 & 38.02 & 3.74 & 53.8 & 3.72 & 50.6 & 3.63 \\
\hline $\begin{array}{l}L_{c}=150 \mathrm{mmd}_{i}=30 \mathrm{~mm} \\
d_{c}=73 \mathrm{~mm}\end{array}$ & 34.8 & 5.78 & 36.3 & 3.85 & 68.3 & 3.51 & 65.8 & 3.38 \\
\hline \multicolumn{9}{|c|}{$d_{o}=42 \mathrm{~mm}$} \\
\hline $\begin{array}{l}L_{c}=100 \mathrm{~mm} d_{i}=51 \mathrm{~mm} \\
d_{c}=73 \mathrm{~mm}\end{array}$ & 56.5 & 4.19 & 48.4 & 3.50 & 42.4 & 3.31 & 47.0 & 3.50 \\
\hline $\begin{array}{l}L_{c}=150 \mathrm{mmd}_{i}=47 \mathrm{~mm} \\
d_{c}=146 \mathrm{~mm}\end{array}$ & 58.1 & 4.07 & 35.3 & 3.65 & 48.7 & 3.60 & 42.7 & 3.61 \\
\hline $\begin{array}{l}L_{c}=25 \mathrm{~mm}, d_{i}=42 \mathrm{~mm} \\
d_{c}=73 \mathrm{~mm}\end{array}$ & 62.3 & 3.98 & 56.0 & 3.35 & 59.3 & 3.44 & 53.9 & 3.44 \\
\hline $\begin{array}{l}L_{c}=50 \mathrm{~mm}, d_{i}=30 \mathrm{~mm} \\
d_{c}=146 \mathrm{~mm}\end{array}$ & 45.5 & 5.05 & 40.1 & 3.62 & 60.7 & 3.44 & 23.3 & 3.69 \\
\hline \multicolumn{9}{|c|}{$d_{o}=30 \mathrm{~mm}$} \\
\hline $\begin{array}{l}L_{c}=150 \mathrm{~mm}_{i}=51 \mathrm{~mm} \\
d_{c}=73 \mathrm{~mm}\end{array}$ & 54.5 & 3.87 & 19.8 & 3.70 & 30.7 & 3.65 & 23.3 & 3.69 \\
\hline $\begin{array}{l}L_{c}=100 \mathrm{~mm} d_{i}=47 \mathrm{~mm} \\
d_{c}=146 \mathrm{~mm}\end{array}$ & 58.6 & 3.69 & 27.4 & 3.63 & 34.5 & 3.60 & 29.5 & 3.62 \\
\hline $\begin{array}{l}L_{c}=50 \mathrm{~mm}, d_{i}=42 \mathrm{~mm} \\
d_{c}=73 \mathrm{~mm}\end{array}$ & 52.4 & 4.00 & 18.8 & 3.81 & 35.6 & 3.63 & 26.8 & 3.60 \\
\hline $\begin{array}{l}L_{c}=25 \mathrm{~mm}, d_{i}=30 \mathrm{~mm} \\
d_{c}=146 \mathrm{~mm}\end{array}$ & 57.9 & 4.08 & 30.9 & 3.59 & 47.8 & 3.50 & 36.4 & 3.56 \\
\hline
\end{tabular}

Figura 5.12: Tabla que contiene los exponentes de la velocidad (b) y la constante (a) obtenidos a través de las medidas y de las diferentes acotaciones de la región fuente:

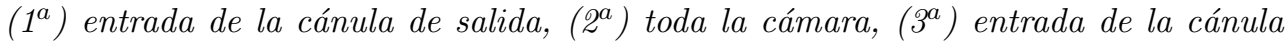
de salida y salida de la cánula de entrada. 


\section{Capítulo 6}

\section{Conclusiones y desarrollos futuros}

6.1 Conclusiones ................... 223

6.2 Desarrollos Futuros ............. 231 



\subsection{Conclusiones}

Las conclusiones del trabajo realizado y presentado en esta tesis doctoral, sobre el estudio y predicción del ruido de flujo generado por sistemas de escape se agrupan del modo siguiente. En primer lugar se justifica, a partir del estado actual del conocimiento en esta materia, la importancia de su estudio así como la metodología empleada para abordarlo con profundidad. En segundo lugar, se exponen las conclusiones relativas al desarrollo de la metodología experimental y al modelo de cálculo propuesto en el último capítulo, necesarios para enfocar un estudio adecuado del ruido de flujo. Finalmente, se comentan los resultados obtenidos de la aplicación de dicha metodología de estudio al conocimiento y cuantificación de los mecanismos de generación de ruido de flujo en sistemas de escape reales.

Dentro del primer bloque de conclusiones se pueden señalar las siguientes:

- Se ha realizado un inventario de las distintas contribuciones que pueden distinguirse en el ruido total emitido por un motor de combustión interna alternativo, con la finalidad de valorar la importancia relativa del ruido aerodinámico de escape en el que se enmarca el ruido de flujo, cuyo estudio y estimación constituyen el principal objetivo de esta tesis doctoral. Entendemos por ruido de flujo el generado por el flujo medio que transita por el sistema de escape y que es emitido a la atmósfera en reposo, componente que se está constituyendo como una fuente sonora cada vez más relevante en la emisión de ruido de escape.

- Una vez centrada la importancia del problema abordado, se han analizado las posibles causas de generación de este tipo de ruido, normalmente asociado a los fenómenos de turbulencia que se generan en diferentes partes del sistema de escape. En particular, se distinguen dos fuentes de generación: (i) la turbulencia generada por el paso del flujo a través de las singularidades encontradas en el línea de escape, y (ii) la generada en la sección de descarga debida a la interacción del flujo saliente con la atmósfera en reposo. Esto nos permitirá plantear el estudio de la generación del ruido de flujo bajo ambas perspectivas y separando sus contribuciones.

- Se han analizado los diferentes comportamientos de las contribuciones al ruido de escape: ruido pulsante y ruido de flujo, planteándose la posibilidad de realizar un estudio desacoplado de ambas componentes bajo ciertas condiciones. 
- Se ha realizado una revisión crítica exhaustiva de los diferentes métodos de cálculo de la generación sonora por la turbulencia, planteándose la necesidad de que todo estudio teórico esté acompañado por un diagnóstico experimental y computacional del mismo, lo que termina por centrar el enfoque escogido en este trabajo.

Una vez establecida la necesidad de desarrollar una metodología de estudio adecuada, centrada básicamente en el diagnóstico experimental de la contribución del ruido de flujo, y en una evaluación computacional que verifique dichos estudios, se ha procedido a su desarrollo, que puede resumirse en los siguientes pasos.

- Se ha planteado la conveniencia de desarrollar metodologías experimentales que permitan separar ambos fenómenos de generación de turbulencia (generaciones internas y externas), a fin de profundizar en el conocimiento de la generación del ruido de flujo.

- En primer lugar, se ha desarrollado un método de diagnóstico experimental que analiza por un lado, los mecanismos de generación de turbulencia durante los procesos de emisión no estacionaria de los gases calientes a la atmósfera en reposo, y, por otro lado, la turbulencia generada internamente por el sistema de escape.

- Debido a que el primer fenómeno, descarga a la atmósfera, es difícilmente controlable y la única posibilidad de reducirlo es escoger adecuadamente los parámetros que lo definen, se ha realizado un estudio experimental sobre el modelo de emisión que gobierna tal fenómeno a fin de establecer qué factores definen el efecto del flujo medio sobre la componente pulsante.

- En este sentido se desarrolla un método de diagnóstico experimental de la fluctuación del caudal volumétrico en la boca de la cánula de escape a partir de la medida de presión en cánula, estableciendo así la fuente sonora que se sitúa en esta sección y evaluando a partir de ella los fenómenos de emisión. Este método permite separar y analizar ambas contribuciones, fuentes internas y externas, y obtener el espectro del ruido radiado al exterior en diferentes posiciones angulares partiendo de una única medida de presión en cánula.

- Se muestra una elevada robustez del método de estimación del caudal volumétrico a través de un análisis de errores mediante el empleo de una simulación numérica, errores que quedan dentro del rango del error 
experimental; lo que permite garantizar que las diferencias observadas con las estimaciones del ruido de escape se deben al modelo de emisión empleado y no a los errores de estimación de la fuente.

- A partir de los resultados experimentales en motor, se plantea la posibilidad de realizar en estudio desacoplado de la componente pulsante y de flujo, en el ruido emitido, de forma que esta segunda componente tiene un carácter próximo a la emisión estacionaria del flujo y se constituye como una fuente sonora adicional que será estudiada de forma aislada.

- Una vez justificada la validez de la aproximación estacionaria para el análisis del ruido de flujo generado por los sistemas de escape a altos regímenes de giro, donde esta contribución es dominante, se desarrolla, en segundo lugar, una metodología experimental (intensimetría en conductos) para la estimación del ruido de flujo generado únicamente por las fuentes internas de elementos simples del sistema de escape; en particular del ruido de flujo generado por el silenciador trasero y la cánula de escape, asumiendo que la turbulencia generada por aquellas partes del sistema próximas al motor son atenuadas por el mismo sistema de escape. Esta metodología ofrece la ventaja de separar contribuciones internas y externas.

- Una vez evaluado el comportamiento espectral de estas fuentes de generación interna, se aborda el diagnóstico de los valores de ruido global emitidos a la atmósfera, por integración de la intensidad emitida para todo el rango de frecuencias audibles, ya que son estos valores a los que se refiere la normativa de emisión acústica vigente. Este estudio se realiza bajo dos perspectivas diferentes:

1. La obtención de leyes de escalado que relacionen el ruido global emitido con las características del flujo (velocidad media) y con las características geométricas del elemento analizado. Dichas leyes permiten establecer criterios de diseño a fin de reducir la contribución sonora del ruido de flujo y evaluar el ruido generado por la misma geometría modificando sus parámetros de diseño sin necesidad de efectuar nuevas medidas.

2. Estudio experimental y computacional del mismo mediante el desarrollo de las analogías acústicas existentes en la literatura.

- El estudio experimental de valores de ruido global emitido y la correspondiente obtención de las leyes de escalado empleando los resultados 
de la técnica de intensimetría en conductos, dan cuenta sólo de la contribución interna y permiten presentar toda la potencia de esta técnica de diagnóstico y estudio de los mecanismos de generación interna de ruido de flujo y la naturaleza de estas fuentes.

- Una vez desarrolladas las metodologías experimentales que permiten realizar comparativas adecuadas con los análisis teóricos existentes en la literatura, se desarrolla un método de cálculo basado en simulaciones computacionales para obtener el comportamiento del ruido de flujo generado por elementos aislados del sistema de escape.

- El método de cálculo propuesto, pretende estimar las contribuciones de las fuentes internas de un sistema al ruido de flujo generado por dicho sistema. Este método permite diagnosticar tendencias de emisión en función de los parámetros geométricos del silenciador y del flujo, e identificar y localizar las fuentes.

Respecto a la aplicación de los métodos a geometrías reales y los resultados obtenidos cabe indicar los siguientes comentarios.

- El método experimental de estimación del ruido de escape ha sido aplicado a una línea de escape real con los resultados siguientes:

1. Se ha validado experimentalmente el procedimiento de estimación del caudal volumétrico en la boca de la cánula de escape como fuente acústica, sensible a la evolución con el régimen de giro y a los cambios en la geometría del sistema de escape.

2. El modelo de emisión de un pistón vibrante es más adecuado que el modelo de emisión monopolar, ya que, el factor de directividad que contiene consigue reproducir de forma más adecuada el espectro radiado para todo el rango de frecuencias medidas, en especial para frecuencias superiores a $300 \mathrm{~Hz}$ donde los efectos del flujo son más importantes.

3. El límite de validez del procedimiento de estimación de la energía acústica radiada queda fijado en valores de $\zeta=\langle\widetilde{u}(t) / U\rangle$ (valor medio del cociente entre la fluctuación de la velocidad en la boca de salida de la cánula de escape, y la velocidad media del flujo) por debajo de 0.5. Esta relación supone una sobrestimación de 
la presión sonora radiada para altas amplitudes de la componente pulsante en comparación con el flujo medio, donde la influencia del comportamiento no lineal del extremo de un conducto sobre las características de reflexión adquiere mayor importancia, existiendo una mayor absorción de la energía acústica por parte de la capa vortical desarrollada en dicho extremo, a lo que también contribuye el fenómeno de reflujo que suele presentarse en estos mismos casos.

4. El método propuesto ofrece la ventaja de poder obtener el espectro de ruido radiado a diferentes distancias y posiciones angulares partiendo de una única medida de presión en la cánula de escape, lo que proporciona una disminución importante del coste experimental, al no ser necesaria una enorme colección de medidas exteriores para conocer el espectro radiado en diversas posiciones.

5. Asimismo, el método permite identificar los mecanismos de generación sonora al separar la contribución de la fuente interna (elementos de la línea de escape) contenida en la estimación de la derivada del caudal volumétrico en la sección de salida de ésta, y las fuentes externas derivadas de la descarga, interacción de esta aceleración del caudal con la atmósfera en reposo.

6. Finalmente, mencionar como última ventaja de este procedimiento de estimación del ruido de escape radiado al exterior, la de poder mejorar aisladamente tanto los modelos de emisión como los cálculos del flujo usados para la predicción de ruido, identificando en cada caso que efecto producen estas mejoras en la estimación del espectro de ruido medido en el exterior.

- La estimación del ruido de flujo generado por un flujo estacionario en elementos aislados del sistema de escape, se ha analizado experimentalmente (a través de la técnica de intensimetría en conductos) y computacionalmente, aplicando ambos procedimientos a una misma geometría simple (cámara de expansión reversa).

- La validación de la técnica de intensimetría en conductos y su potencia se ha evaluado desde el punto de vista de vista de predicción espectral y de la obtención de los niveles globales de ruido emitido, extrayéndose las siguientes conclusiones: 
1. La técnica de intensimetría en conductos se muestra como una técnica experimental adecuada y potente para el estudio del ruido de flujo generado por elementos simples del sistema de escape, tanto desde el punto de vista de predicción espectral como de obtención de los niveles globales de ruido. Presentando la cualidad de permitir analizar los mecanismos de generación interna y su relación con los espectros externos medidos, proporcionando así una guía útil no sólo, para la identificación de estos mecanismos de generación, sino también, para introducir las debidas modificaciones en la fuente generadora del ruido de flujo actuando sobre sus parámetros geométricos, con el fin de reducir su contribución al espectro de ruido radiado.

2. El sonido generado por la fuente interna a bajas frecuencias no es radiado al campo libre; además, dicho flujo de energía no radiada aumenta al aumentar el flujo medio uniforme en la cánula (aumento del gasto y por tanto de la velocidad del flujo).

3. Los efectos de la descarga debidos a la capa de inestabilidad que se genera en la boca del escape y que se comporta como una fuente de ruido secundaria tienen una contribución más importante a alta frecuencia y pueden modelarse mediante una eficiencia de radiación igual al número de Strouhal, $S_{t}$, del flujo estacionario considerado, adoptando como longitud característica de este proceso de emisión el radio de la cánula de salida.

4. Los efectos del ruido de flujo radiado son más importantes a partir de unos $400 \mathrm{~Hz}$, lo que está en concordancia con los resultados experimentales obtenidos para los espectros del ruido de escape emitido por un motor.

- Del análisis experimental de leyes de escalado derivadas de la estimación de globales relacionadas con las analogías acústicas cabe citar:

1. Este tipo de análisis presenta a la intensimetría en conductos como una herramienta experimental de gran utilidad para el estudio del ruido de flujo generado por los elementos del sistema de escape, proporcionando un mayor conocimiento de los mecanismos de generación interna, comportamiento de la fuente, y por lo tanto, aportando criterios de diseño de utilidad en la industria. 
2. Esta metodología de estudio del ruido de flujo es idónea para aquellos elementos de escape que presenten una generación sonora de ruido de flujo superior a la de descarga de un chorro libre, geometrías más relevantes desde el punto de vista del análisis de fuentes internas, ya que en el caso de geometrías cuyo sonido radiado sea del orden de la descarga de un chorro libre el efecto de las fuentes internas es despreciable en la medida exterior, y por tanto su análisis es de escaso interés para la industria. En estas geometrías (generación interna importante) las leyes de escalado derivadas a partir de las medidas de intensimetría en conductos, reproducen los efectos observados en las medidas de intensidad radiada en una cámara semianecoica.

3. Para poder extraer la máxima información de este método de análisis del comportamiento de las fuentes internas del silenciador, es importante acompañar todos estos resultados experimentales con simulaciones numéricas estacionarias.

4. La leyes de escalado obtenidas permiten extrapolar la predicción de la energía sonora generada por geometrías similares a la analizadas, cuando se efectúan cambios en su geometría o características del flujo, sin necesidad de realizar medidas adicionales, siendo ésta otra de las ventajas que ofrece el método ya que reduce el coste experimental.

- Finalmente de la aplicación, a la misma geometría simple anterior, del método de cálculo del ruido de flujo desarrollado a partir de simulaciones computacionales y considerando las analogías acústicas existentes en la literatura se deduce que:

1. En el caso de no ser conocidas con exactitud las principales fuentes internas, se obtienen resultados aceptables al considerar todas las posibles fuentes presentes en el silenciador (integración a toda la cámara), a pesar de que la hipótesis de compacidad de la fuente no sea satisfecha. Sin embargo, es importante conocer la posición de las principales fuentes internas del silenciador para obtener mejores resultados a través del procedimiento propuesto. En este sentido, el método geométrico escogido para la definición de las fuentes, asociadas a los máximos de las distribuciones espaciales de energía cinética en el interior del silenciador resulta adecuado. 
2. Este método diagnostica las tendencias experimentales observadas en diferentes silenciadores aportando mayor información sobre los fenómenos físicos asociados a la generación de ruido de flujo y ayudando a la identificación de las fuentes de generación interna.

3. En relación a las leyes de escalado, no se reproducen con exactitud las tendencias ni exponentes obtenidos a partir de las medidas, aunque sí que proporcionan una información aproximada del comportamiento real de la fuente en el campo libre.

4. Finalmente podemos concluir diciendo que el modelo de cálculo propuesto a partir de la simulación computacional del campo de velocidades en el interior del silenciador, es una metodología potente que complementa las medidas experimentales, ayudando al conocimiento de los principales mecanismos de generación sonora en el interior del silenciador a través de la identificación de las fuentes sonoras.

Como conclusión general cabe mencionar que se ha establecido una metodología adecuada para el estudio y predicción del ruido de flujo generado durante el proceso de escape en el motor, que aborda diferentes aspectos de su generación y proporciona información relevante para la industria automovilística. Ésta puede resumirse como sigue:

- Se establecen dos metodologías de análisis experimental: (1) estimación del ruido de escape en motor a través de la media de presión en cánula e (2) intensimetría en conductos para el estudio de la generación del ruido de flujo estacionario. De modo, que en una primera fase se determina el efecto del flujo sobre la componente pulsante a través del modelo de emisión sonora, que justifica la posibilidad de realizar un análisis estacionario del ruido de flujo emitido. Factor que es validado y que por tanto puede introducirse en modelos de predicción de ruido de escape a fin de perfeccionarlos. En una segunda fase se estudia la generación del ruido de flujo generado por elementos simples del sistema de escape, desarrollando una metodología experimental basada en medidas estacionarias en flujo frío.

- Ambas metodologías experimentales separan las contribuciones de las fuentes internas y externas, lo cual es necesario para establecer criterios 
de actuación sobre las mismas, en especial sobre las internas, ya que son éstas las que dependen de la geometría de la línea de escape y, por tanto, son más fácilmente modificables.

- Se profundiza en los mecanismos de generación del ruido de flujo estableciendo un estudio de leyes de escalado a través del uso de analogías acústicas de las que se pueden extraer criterios de diseño a fin de reducir la emisión, objetivo final de las industrias relacionadas con el diseño de silenciadores.

- Se desarrolla un modelo de cálculo basado en simulaciones computacionales, que proporciona importante información sobre las principales fuentes sonoras en el interior del silenciador, diagnosticando las tendencias de emisión sonora, a través de la identificación de las fuentes principales de generación sonora en el interior del silenciador, lo que ayudará al desarrollo de planes de ensayos y puede, por tanto, reducir el tiempo de ensayos experimentales.

\subsection{Desarrollos Futuros}

Existen diversos puntos en los estudios realizados que pueden ser mejorados, así como nuevas posibilidades de estudio que pueden resumirse en:

- Introducción en el modelo de emisión presentado en el capítulo 2 de un factor que tenga en cuenta la absorción sonora que se produce para valores de $\zeta$ superiores a 0.5 , asociados a los efectos de no linealidad y reflujo producidos en estos casos.

- En el ámbito computacional del modelo propuesto, este puede mejorarse introduciendo el modelo de propagación adecuado empleando las funciones de Green correctas para la propagación en el interior del silenciador, y realizando simulaciones que contengan también la emisión del flujo a la atmósfera en reposo. Factores que introducirán previsiblemente mejoras al cálculo actual pero que en contrapartida supondrán un coste computacional superior al actual. 



\section{Apéndice A}

\section{Analogías acústicas}

A.1 Teoría de la generación de ruido por vórtices de Powell ....................... 235

A.2 Contribución de Howe al ruido aerodinámico . . 237

A.3 Teoría acústica de Ribner . . . . . . . . . 239

A.4 Generalización acústica propuesta por Doak . . 240

A.5 Ecuación de Lilley . . . . . . . . . . . . . . . . 244 



\section{A.1 Teoría de la generación de ruido por vórtices de Powell}

En la formulación original de la analogía acústica de Lighthill [102] [103] las fuentes de sonido son descritas en términos de una transferencia de momento fluctuante, lo cual en principio y siguiendo la descripción de estructuras de flujo turbulento, y tal y como afirma Davies [43], puede ser evaluado a partir de los promedios temporales obtenidos experimentalmente. Asumiendo en el desarrollo del flujo de corte la predominancia de las componentes solenoidales de la fluctuación de velocidad (vorticidad), Powell [139] desarrolló una formulación alternativa a la de Lighthill describiendo las correspondientes fuentes acústicas en términos de fluctuaciones de vorticidad.

El objetivo de Powell era llegar a una ecuación como la de Lighthill pero invariante Galileana. Para ello propone una expresión alternativa del término fuente $T_{i j}=\rho_{o} u_{i} u_{j}$, propuesto por Lighthill, de forma que éste sea invariante Galileano, aunque al trabajar así, el término de la izquierda de la ecuación de propagación sonora resulte no invariante. La ecuación adoptada por Powell [139] adquiere la siguiente forma

$$
\nabla^{2} p-\frac{1}{a_{o}^{2}} \frac{\partial^{2} p}{\partial t^{2}}=-\nabla\left[\rho \mathcal{L}+\nabla\left(\frac{1}{2} \rho u^{2}\right)-\mathbf{u} \partial \rho / \partial t-\frac{1}{2} u^{2} \nabla \rho\right]
$$

donde se ha asumido que la acción a través del fluido es isoentrópica $(\nabla p \simeq$ $\left.a_{o}^{2} \nabla \rho\right)$. En esta expresión el vector de Lamb $\mathcal{L}=\boldsymbol{\Omega} \times \mathbf{u}$ puede considerarse como la fuerza de Kutta-Joukowski (fuerza de sustentación) sobre el elemento lineal del material, pudiendo aparecer como pares que se cancelan entre sí, si el momento resultante se conserva [32].

Considerando que las variaciones de densidad son debidas únicamente a la compresión cinética, es decir, son del orden de $\rho_{o} M^{2}$, donde $M=U / a_{o}$ es el número de Mach del fluido, los términos fuente de la ecuación (A.1) atendiendo a su orden de magnitud, adquieren las siguientes razones entre sí $1: 1: S_{t} M^{2}: M^{2}$, siendo $S_{t}$ el número de Strouhal. Esto indica que en la forma final del término fuente, al menos uno de los dos primeros términos no se degrada, siendo posible, para flujos subsónicos de baja velocidad $\left(S_{t} M^{2} \ll 1\right.$ y $\left.M^{2} \ll 1\right)$, despreciar con seguridad los dos últimos términos. Bajo estas consideraciones la ecuación anterior puede rescribirse como

$$
\nabla^{2} p-\frac{1}{a_{o}^{2}} \frac{\partial^{2} p}{\partial t^{2}}=-\rho_{o} \nabla\left[\mathcal{L}+\nabla\left(\frac{u^{2}}{2}\right)\right]
$$


de manera que el término fuente propuesto por Powell queda expresado como

$$
Q_{\beta}=\rho_{o} \nabla\left[\mathcal{L}+\nabla\left(\frac{u^{2}}{2}\right)\right]
$$

Suponiendo, además, que en un dominio infinito el segundo miembro del término fuente produce un campo sonoro lejano de menor intensidad que el asociado al primer término, este puede omitirse y la expresión del término fuente adopta la siguiente forma

$$
Q_{\beta} \approx \rho_{o} \nabla \mathcal{L}=\rho_{o} \nabla(\boldsymbol{\Omega} \times \mathbf{u})=\rho_{o} \nabla[(\nabla \times \mathbf{u}) \times \mathbf{u}]
$$

Sin embargo, esta última consideración defendida por Powell [139] para números de Mach y de Strouhal característicos de flujos subsónicos, $S_{t} M \ll 1$, no está suficientemente justificada, ya que en un flujo real ambos términos pueden ser del mismo orden de magnitud, de tal modo que su contribución al campo acústico lejano puede ser comparable (Fedorchenko, [63]) y por tanto deberían considerarse ambos factores.
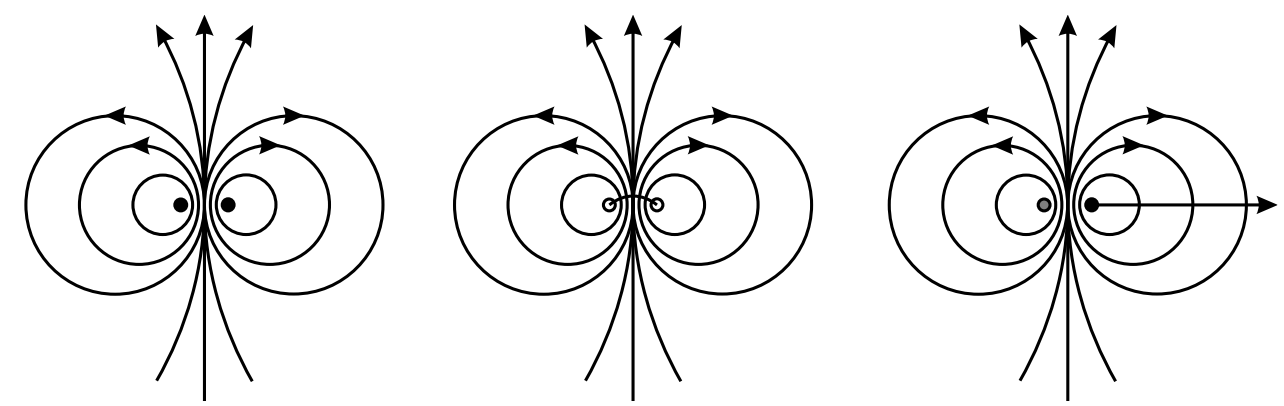

Figura A.1: En esta figura se puede observar, a la izquierda: las lineas de corriente de un campo de flujo debido a un par de vórtices. En el centro: las lineas de corriente debido a una hoja de dipolos. Y a la derecha: las lineas de corriente debidas a un salto vortical sobre un cilindro y acelerando abandonando al vórtice (flujo medio no considerado).

La expresión (A.4), que contiene la vorticidad como la componente generadora de sonido, fue interpretada por Powell como 'una fuente de sonido de carácter dipolar', ver figura A.1.

La idea de Powell, tal como nos recuerda Möhring [118], evoca uno de los mayores esfuerzos para reducir cualquier problema de generación sonora en un flujo subsónico incompresible, únicamente al análisis de la evolución de 
la vorticidad dentro del flujo. Flujo donde es válida la ecuación de vórtices de Helmholtz $\partial \boldsymbol{\Omega} / \partial t+\nabla \times \mathcal{L}=0$, que permite relacionar las componentes de la velocidad del término del tensor de Lighthill $\rho_{o} u_{i} u_{j}$, con la vorticidad generada.

En esta teoría de generación de sonido por vorticidad, la fuente puede expresarse completamente en función de la vorticidad, haciendo uso de la ley de Biot-Savart [81]

$$
\mathbf{u}(\mathbf{r}, t)=\frac{1}{4 \pi} \cdot \nabla \times \int \frac{\Omega\left(\mathbf{r}^{\prime}, t\right)}{\left|\mathbf{r}-\mathbf{r}^{\prime}\right|} d^{3} \mathbf{r}^{\prime}
$$

pero implica el empleo de una función de Green de dificil interpretación [118]. Sin embargo, las fuentes que presenta esta teoría no están localmente relacionadas con la vorticidad, tal como apunta Crighton [32]; de hecho, estas relaciones son no-locales y no lineales en su relación con la vorticidad como ponen de manifiesto, a través de $\mathcal{L}$, las ecuaciones (A.4) y (A.5). La forma de relacionar el campo sonoro linealmente con la vorticidad se debe a Möhring [118], quien no sólo transformó la expresión de la fuente $Q_{\beta}$ empleando las leyes de vórtices no lineales de Helmholtz, sino que transformó las funciones de Green con las cuales se resuelve la fuente. Posteriormente, Powell [140] [141], presentó varios trabajos en los que se proponían argumentos físicos simples para probar la equivalencia entre la formulación de Möhring y la teoría clásica de los vórtices sonoros.

De este modo, esta versión de la analogía de Lighthill abrió el camino a la engañosa idea de conectar las fuentes de sonido aerodinámico exclusivamente con la región de vorticidad no nula, al menos para flujos subsónicos y aproximadamente homoentrópicos. Cualitativamente esto puede sostenerse sólo en algunos aspectos, ya que en la región del flujo donde se concentra la vorticidad tienen lugar fuertes efectos de inestabilidad [63], y efectivamente la estructura del flujo de fondo puede cambiar allí, rápidamente, proporcionando un aumento de la generación sonora. Según Howe [76] el punto de vista de Powell resulta atrayente, ya que la idea de que las fuentes acústicas estén asociadas con regiones del flujo donde la vorticidad no se anule es preferible a la región hidrodinámica más extensa que consideraba Lighthill en su analogía.

\section{A.2 Contribución de Howe al ruido aerodinámico}

Una década más tarde a la aparición de la formulación de Powell, ésta fue reformulada por Howe [76], quién consideró la entalpía de parada, $h_{o}=$ 
$h+\left(1 / 2 \cdot u^{2}\right)$ (donde $h$ representa la entalpía específica del fluido), como la cantidad acústica propagada (variable termodinámica natural que representa el campo acústico), en lugar de la densidad o la presión [43]. Esto proporciona la base de una nueva analogía acústica en la que Howe [76] asume que los términos acústicos están asociados únicamente con las regiones del flujo donde el vector vorticidad y el gradiente de entropía no se anulan. Según el propio autor, esta teoría aparece para resolver aquellos problemas en los que la teoría de Powell presenta algunos inconvenientes, como por ejemplo el estudio de la generación sonora durante la convección inestable de las inhomogeneidades del flujo en conductos, y la debida a la presencia de cuerpos rígidos en el espacio libre.

En esta extensión de la teoría de Powell se tienen en cuenta las variaciones de entropía, substituyendo así el medio en reposo considerado por Lighthill y Powell por un flujo medio irrotacional estable [76]. La ecuación de la analogía acústica de Howe en términos de la entalpía de parada, $h_{o}$, corresponde a

$$
\frac{1}{a_{o}^{2}} \frac{\partial^{2} h_{o}}{\partial t^{2}}-\nabla^{2} h_{o}=\nabla(\boldsymbol{\Omega} \times \mathbf{u}-T \nabla s)
$$

De donde se extrae la expresión del término fuente

$$
q=\nabla \mathcal{L}
$$

donde ahora el vector de Lamb adopta la forma $\mathcal{L}=\boldsymbol{\Omega} \times \mathbf{u}-T \nabla s$, siendo $s$ la entropía específica. En esta expresión del término fuente (A.7), el primer término del vector de Lamb evalúa la generación sonora por efectos dinámicos (en particular, debido a esfuerzos de cizalla), y el segundo término evalúa el efecto de la diferencia de temperatura entre el flujo y su entorno, así como las diferencias de entropía, como se puede deducir del desarrollo de este segundo término

$$
\nabla(T \cdot \nabla s)=\nabla T \cdot \nabla s+T \cdot \nabla^{2} s
$$

La divergencia simple en la expresión de la fuente, indica nuevamente un carácter dipolar, de tal manera que estos dipolos ofrecen una estructura cuadripolar oculta que debe tenerse cuidadosamente en cuenta para evitar sobrestimaciones en ciertas ocasiones.

Las situaciones en las que esta fuente describe más adecuadamente los fenómenos físicos involucrados en la generación de ruido aerodinámico son aquéllas en las que no es permisible despreciar la transferencia de calor en el fluido (A.8), aunque sí que es posible asumir que los efectos viscosos son poco 
importantes en la región fuente. Por ello, esta teoría se considera la más apropiada para el estudio del sonido radiado por motores de combustión interna, ya que el término $\nabla(T \cdot \nabla s)$ debe tenerse en cuenta cuando se analiza la evolución de un flujo de fondo no estacionario, a pesar de que Fedorchenko [63] sostiene que no existe ningún motivo para que éste sea tratado como una fuente sonora. Uno de los defectos atribuidos por este último autor a la presente teoría es la imposibilidad de aclarar cuáles de los términos en la ecuación (A.7) deben atribuirse únicamente al proceso de generación sonora por el flujo, ya que no existe ninguna forma de distinguir entre el campo acústico y el flujo de fondo inestable. En cualquier caso, Fedorchenko también afirma que no se puede considerar una ecuación diferencial de segundo orden escalar que contenga un conjunto de variables totales desconocidas, como una fuente sonora radiando o no, cuando no se ha considerado un adecuado procedimiento de descomposición del flujo.

\section{A.3 Teoría acústica de Ribner}

Ribner consideró una nueva variable natural del campo acústico $p_{\omega}=$ $p^{\prime}-p^{(0)}$, que es interpretada como una 'presión acústica'; de esta forma, la ecuación de propagación sonora para el caso de un flujo subsónico homoentrópico sin fuerzas externas queda modificada y expresada de la forma

$$
\frac{1}{a_{o}^{2}} \frac{\partial^{2} p_{\omega}}{\partial t^{2}}-\nabla^{2} p_{\omega}=-\frac{1}{a_{o}^{2}} \frac{\partial^{2} p^{(0)}}{\partial t^{2}}
$$

donde el término fuente se dice que es 'una fuente sonora de tipo monopolar' y $p^{(0)}$ es la pseudo-presión sonora de Ribner dada por la expresión: $\nabla^{2} p^{(0)}=$ $-\partial^{2} T_{i j} / \partial x_{i} \partial x_{j}$. Uno de los rasgos más curiosos de esta teoría es el hecho de que el término fuente no dependa explícitamente de la velocidad del flujo incompresible, y sólo se consideren decisivos los cambios temporales en la variable escalar $p^{(0)}$. Esto contradice la opinión común, defendida por Powell [139], Howe [76] y Möhring [118], de que un flujo irrotacional inestable es incapaz de producir fuentes sonoras.

Normalmente esta aproximación ha sido criticada y es por ello la menos popular de todas las teorías acústicas mencionadas. En este sentido, la aseveración realizada por Möhring [118] (y posteriormente por Crighthon [32]) sobre el decaimiento de $p^{(0)}$ como $|\mathbf{r}|^{-3}$ cuando no existen fuentes de masa ni fuerzas en la región de flujo, y la derivación de la fuente hacia una integral divergente 
es, en este caso, incorrecta si se considera la regla matemática elemental

$$
\int \frac{\partial^{2} p^{(0)}}{\partial t^{2}} d x_{1} d x_{2} d x_{3}=\frac{\partial^{2}}{\partial t^{2}} \int p^{(0)} d x_{1} d x_{2} d x_{3}
$$

que es únicamente válida si ambas integrales existen y son uniformemente convergentes, siendo posible que la integral de la izquierda que define la tensión total del término fuente, sea convergente mientras que la integral de $p^{(0)}$ sea divergente. Es decir, a pesar de que generalmente el comportamiento de este término fuente es infinito, para cada situación se exige un análisis más preciso. De cualquier modo, según Fedorchenko [63], esta teoría continúa cayendo en la misma línea de los errores discutidos en la analogía de Lighthill, puesto que sigue suponiendo que una 'ecuación de ondas inhomogénea' escalar que separa el término fuente de la propagación sonora, es suficiente para la definición aerodinámica de las fuentes sonoras, sin proporcionar ninguna idea sobre la separación adecuada de las componentes acústicas.

La opinión actual universalmente aceptada (Crighthon [32] y Ffowcs Williams [64]) es que cualquier tipo de fuente sonora (monopolar, dipolar o cuadripolar) definida en la gran variedad de teorías existentes, es 'igualmente exacta' y difiere únicamente en sus términos no radiantes, los cuales proporcionan contribuciones nulas en el campo sonoro lejano.

La base de la extensión realizada por Ribner [149] para la consideración de los efectos del flujo en su teoría, radica en el reemplazamiento de la función de Green oscilatoria normal por aquélla correspondiente a la ecuación de onda convectiva, lo que permite un desacoplamiento entre las fuentes y las ondas sonoras.

\section{A.4 Generalización acústica propuesta por Doak}

Recientemente, Doak [56] ha presentado una formulación general rigurosa que proporciona una estricta confirmación de la identificación de Powell del rol frecuentemente predominante de la vorticidad en la generación de ruido aerodinámico.

Doak empleó un promediado temporal sobre un intervalo $\tau$, que puede seleccionarse arbitrariamente, para definir una fluctuación de entalpía de parada [56], $h_{o}^{\prime}$, como la variable básica del campo acústico, derivando una ecuación de onda escalar inhomogénea de segundo orden de tipo convectivo lineal en $h_{o}^{\prime}$. En un sentido amplio, esta ecuación intenta desarrollar los conceptos de Howe, pero su forma difiere de esta última en varios aspectos. 
Doak, al igual que Howe, pretende proponer un modelo matemático alternativo que sea físicamente más significativo en relación a aquellos términos que representan refracción y/u otros procesos locales relevantes en la generación sonora que ocurren realmente en el fluido, de forma que estos sean expuestos explícitamente. Estos procesos eran representados en la analogía de Lighthill por un conjunto redundante de 'fuentes cuadripolares' en un medio 'acústico clásico', en el cual la velocidad de las ondas sonoras tiene un valor constante y apropiado para el fluido del campo lejano. En la opinión de Doak [56] ninguno de estos modelos alternativos, incluido el suyo, ha proporcionado una representación matemática explícita satisfactoria de este proceso. Éste constituye un rasgo general de todos estos modelos; sin embargo, el desarrollo de diferentes campos acústicos, además del propuesto por Lighthill, hace evolucionar el concepto generalizado de campo acústico, considerando estos intentos como parte de la búsqueda general de tal campo.

Basados en la experiencia, Doak [56] proporciona los siguientes criterios que debe verificar el campo acústico: (i) La ecuación que lo describa debe ser exacta, dadas las ecuaciones de transporte básicas y las relaciones termodinámicas del fluido referido. (ii) Todas las funciones campo consideradas y sus productos deben ser representadas explícitamente como suma de una parte media (promediado-temporal) bien definida y partes fluctuantes. (iii) La ecuación debe ser una ecuación de onda escalar inhomogénea de tipo convectivo (siendo la velocidad de onda, partícula a partícula, la velocidad acústica local de onda) la cuál es explícitamente lineal en la dependencia fluctuante del campo y se reduce claramente a la ecuación de D'Alembert para la fluctuación de presión de pequeña amplitud (movimiento adiabático fluctuante) cuando el fluido está en reposo. (iv) Además cada uno de los términos homogéneos e inhomogéneos en la ecuación deben representar individualmente todos los procesos físicos locales relevantes y relativamente distintos implicados en el movimiento, los cuales son tan distintos unos de otros como tan posibles de medir individualmente en la práctica.

Con respecto a la verificación de estos criterios, Doak [56] manifiesta que únicamente la fluctuación de la entalpía de parada $h_{o}^{\prime}$, si es considerada como campo acústico, permite la linealización particular parcial de la ecuación del momento. Por otro lado, ésta conduce a una ecuación de onda escalar inhomogénea de tipo convectivo (criterio (iii)), en la cual la diferencia requerida entre los términos por el criterio (iv) aparece también 'maximizada' en una extensión significativa. Además, la exactitud matemática especificada en el criterio (i) se refiere a que ninguna de las cantidades consideradas en las ecuaciones básicas, que no son constantes físicas 'absolutas', sea tratada como coordenadas independientes en el espacio y en el tiempo. 
De acuerdo con los comentarios anteriores la ecuación de onda escalar inhomogénea de segundo orden que fue derivada por Doak para la fluctuación de entalpía de parada adopta la siguiente expresión

$$
\begin{gathered}
\frac{\partial^{2} h_{o}^{\prime}}{\partial x_{i}^{2}}-\left[\frac{1}{c^{2}}\left\{\frac{\partial^{2} h_{o}^{\prime}}{\partial t^{2}}+\left(2 u_{i} \frac{\partial}{\partial t}-(\mathbf{u} \times \boldsymbol{\Omega})_{i}+\mathfrak{V}_{i}-2 \frac{\partial h}{\partial x_{i}}\right) \frac{\partial h_{o}^{\prime}}{\partial x_{i}}+u_{i} u_{j} \frac{\partial^{2} h_{o}^{\prime}}{\partial x_{i} \partial x_{j}}\right\}\right]^{\prime} \\
=\frac{\partial}{\partial x_{i}}\left[(\mathbf{u} \times \boldsymbol{\Omega})_{i}^{\prime}+\mathfrak{V}_{i}^{\prime}\right]-\left[\begin{array}{c}
\left.\frac{1}{c^{2}}\left(-(\mathbf{u} \times \boldsymbol{\Omega})_{i}+\mathfrak{V}_{i}-2 \frac{\partial h}{\partial x_{i}}+u_{i} u_{j} \frac{\partial}{\partial x_{j}}\right) \cdot\right]^{\prime} \\
\cdot\left(\left[(\mathbf{u} \times \boldsymbol{\Omega})_{i}^{\prime}+\mathfrak{V}_{i}^{\prime}\right]+\left[\frac{\partial u_{i}^{\prime}}{\partial t}\right]\right)
\end{array}\right]+ \\
+\left[\frac{\partial}{\partial t}\left(\frac{1}{c^{2}}\right)^{\prime} \frac{D h}{D t}\right]^{\prime}-\left[\frac{\partial}{\partial t}\left(\frac{1}{R} \frac{D s}{D t}\right)^{\prime}\right]^{\prime}-\left[\frac{1}{c^{2}} u_{i} \frac{\partial \mathfrak{V}_{i}^{\prime}}{\partial t}\right]^{\prime}
\end{gathered}
$$

donde $\boldsymbol{\Omega}$ es la vorticidad, $c$ la velocidad del sonido $\left(c^{2} \equiv \gamma R T\right), D / D t \equiv \partial / \partial t+$ $u_{i} \partial / \partial x_{i}$ es la derivada material, $\mathfrak{V}_{i} \equiv T \partial s / \partial x_{i}+(1 / \rho) \partial \sigma_{i j} / \partial x_{j}+f_{i}$ es la suma de aceleraciones, siendo en ella $T$ la temperatura, $s$ la entropía del fluido, $f_{i}$ la fuerza externa por unidad de masa y $\sigma_{i j}$ es el tensor de esfuerzos viscosos, que está relacionado con el tensor de esfuerzos compresivos $p_{i j}$ mediante la ecuación (4.21). En esta expresión $\overline{\left[\frac{\partial u_{i}^{\prime}}{\partial t}\right]}$ representa la "aceleración media", que es cero si la fluctuación de velocidad es estacionaria sobre el intervalo temporal considerado en el promediado. Se puede apreciar en el término de aceleración $\mathfrak{V}_{i}$ que la primera aceleración se anula si el movimiento es homoentrópico, la segunda se anula si el fluido es no viscoso y la tercera se anula si no existen fuerzas externas.

El miembro de la derecha de la ecuación (A.10), que contiene la forma del término fuente, consiste en una expresión diferencial no lineal de segundo orden incluyendo las principales variables en sus fluctuaciones (en medio de los cuales aparece la aceleración de Coriolis $\mathbf{u} \times \boldsymbol{\Omega}$ ), así como las fuentes asignadas externamente.

Fedorchenko [63], con respecto a la consideración de $h_{o}^{\prime}$ como el 'campo acústico básico generalizado', afirma que es necesario tener en cuenta que estas ondas de $h_{o}^{\prime}$ 'no son necesariamente ondas acústicas, o incluso ondas en el sentido clásico; sin embargo también afirma que cuando las perturbaciones de $h_{o}^{\prime}$ alcanzan una región de fluido, aparte de la densidad de masa y temperatura uniforme, y aparte del reposo y movimiento uniforme, ellas se convierten en ondas acústicas clásicas de fluctuación de presión'.

Doak [56] hace hincapié, sin embargo, en un serio defecto de su ecuación (A.10), que es la 'redundancia' matemática, similar a aquélla que está presente en las aproximaciones de Lighthill y Howe. Efectivamente, $h_{o}^{\prime}=h^{\prime}+\left(\mathbf{u}^{\prime 2} / 2\right)$, y $h^{\prime}$ y $u_{i}$ aparecen ambos en los términos de la parte de la izquierda y en los de la parte de la derecha. 
La consideración de la entalpía como variable natural del campo acústico permite a Doak derivar una ecuación de la divergencia de la intensidad acústica media correspondiente, $\overline{h_{o}^{\prime} m_{i}^{\prime}}$, donde $m_{i}=\rho u_{i}$ es el campo de momento lineal. Teniendo en cuenta las siguientes consideraciones $\overline{m_{i}} \overline{(\mathbf{m} \times \varpi)}{ }_{i}={\overline{m_{i}}}_{\overline{\left(\mathbf{m}^{\prime} \times \varpi\right)}}=$ $\overline{m_{i}} \overline{\left(\mathbf{m}^{\prime} \times \varpi^{\prime}\right)_{i}}$, donde $\varpi_{i}=\Omega_{i} / \rho$ representa la vorticidad de Beltrami y $\overline{h_{o} m_{i}}=$ $\overline{h_{o}} \overline{m_{i}}+\overline{h_{o}^{\prime} m_{i}^{\prime}}$, Doak [56] obtuvo la siguiente expresión

$$
\begin{aligned}
\frac{\partial\left(\overline{h_{o}^{\prime} m_{i}^{\prime}}\right)}{\partial x_{i}}= & \overline{m_{i} \mathfrak{V}_{i}}+\overline{\rho T \frac{\partial s^{\prime}}{\partial t}}-\overline{\frac{\partial\left(\rho h_{o}-p\right)}{\partial t}}-\overline{m_{i}}\left[\overline{\mathfrak{V}_{i}}-\overline{\left(\mathbf{m}^{\prime} \times \varpi^{\prime}\right)_{i}}+\overline{\left(\frac{\partial u_{i}^{\prime}}{\partial t}\right)}\right]+ \\
+\overline{h_{o}} \overline{\left[\frac{\partial \rho^{\prime}}{\partial t}\right]=} & \overline{m_{i}^{\prime}\left[\left(\mathbf{m}^{\prime} \times \varpi^{\prime}\right)_{i}+\mathfrak{V}_{i}^{\prime}\right]}+\rho T \overline{\frac{\partial s^{\prime}}{\partial t}}-\overline{\frac{\partial}{\partial t}\left(\rho h_{o}-p\right)^{\prime}}+ \\
& +\overline{m_{i}} \overline{\left[\frac{\partial u_{i}^{\prime}}{\partial t}\right]}+\bar{B}\left[\overline{\left.\frac{\left.\partial \rho^{\prime}\right]}{\partial t}\right]}\right.
\end{aligned}
$$

donde $\frac{\partial}{\partial t}\left(\rho h_{o}-p\right)$ representa la velocidad de cambio de la energía total por unidad de volumen. Respecto a esta ecuación debe recordarse que sobre un intervalo temporal a lo largo de toda la duración de las perturbaciones del flujo, es decir, desde el tiempo en que la región de fluido considerada parte de una condición de equilibrio uniforme hasta el instante en el cual vuelve a ella (o se aproxima a ella), la estacionariedad temporal del flujo está asegurada, así como también para fluctuaciones periódicas o fluctuaciones que pueden modelarse como promedios. Para tales flujos, la versión estacionaria de (A.11) conduce a un método matemático simple para el cálculo de la potencia acústica radiada de sonido generado aerodinámicamente por fuentes sonoras puntuales [56].

La conclusión más importante de la teoría de Doak se refiere a la evidencia matemática de que la fluctuación de entalpía $h_{o}^{\prime}\left(x_{k}, t\right)$, en lugar de la fluctuación de presión, representa el campo acústico de cualquier movimiento fluctuante de un fluido, idea que también defendía Howe. De esta forma Doak considera que se ha avanzado considerablemente en la comprensión de la dinámica local involucrada en la generación y radiación del sonido generado aerodinámicamente, dinámica que es a su vez relevante para el caso de flujos turbulentos de fluidos compresibles.

Con respecto a los defectos de esta teoría, Fedorchenko [63] menciona el hecho de que en este nuevo modelo se vuelve a realizar una separación del miembro de la izquierda (que consiste en una especie de "operador de propagación de onda" sobre una única variable) como una única ecuación escalar de segundo orden que contiene todas las variables del flujo, considerando que esta no es una base suficiente para tratar el miembro de la derecha como una 
"fuente sonora", siendo éste además una expresión diferencial no lineal de segundo orden. Por otro lado, Fedorchenko considera que aunque es posible aceptar que el término no lineal de la aceleración de Coriolis $(\mathbf{u} \times \boldsymbol{\Omega})$ puede ser particularmente importante para muchos flujos reales, es generalmente dificil de aceptar la opinión sostenida por Doak [56] de que 'se ha convertido en un reconocimiento cada vez mayor del papel que juega esta aceleración que a menudo domina la representación de la generación de sonido aerodinámico'.

\section{A.5 Ecuación de Lilley}

La ecuación de Lighthill fue modificada por Lilley para facilitar la separación de los términos de propagación sonora y los términos fuente (Béchara [11]), ya que una de las limitaciones de la analogía acústica de Lighthill, señalada por Lilley [106], es el hecho de que en ella las fuentes sonoras equivalentes puedan moverse, pero no el fluido.

La ecuación de Lilley se considera como un desarrollo de la ecuación de Lighthill, que permite evaluar la covarianza temporal de cuarto orden que aparece en su analogía, para la determinación de la potencia acústica sonora radiada. Para ello Lilley [106] parte de las ecuaciones de conservación del fluido (conservación de masa, momento y energía) para el caso particular de gas Newtoniano compresible, escribiéndolas de la siguiente forma

$$
\begin{gathered}
\frac{\partial \rho}{\partial t}+\nabla(\rho \mathbf{u})=0 \\
\frac{\partial}{\partial t}(\rho \mathbf{u})+\nabla(\rho \mathbf{u u})=-\nabla p+\nabla \sigma \\
\frac{\partial}{\partial t}\left(\rho h_{o}\right)+\nabla\left(\rho \mathbf{u} h_{o}\right)-\frac{\partial p}{\partial t}=\nabla(\amalg+\sigma)
\end{gathered}
$$

donde $\sigma$ es el tensor de tensión de cizalla, $\amalg$ es el vector de flujo de calor y $h_{o}=h+u^{2} / 2$, donde $h$ es la entalpía especifica, la cual en un gas perfecto viene dada por $c_{p} T$, siendo $c_{p}$ el calor específico a presión constante y $T$ la temperatura absoluta.

Esta última ecuación de conservación de la energía (A.14) puede expresarse en términos de la entropía $s$ empleando la definición de la derivada convectiva $D / D t \equiv \partial / \partial t+\mathbf{u} \cdot \nabla[55]$, del siguiente modo [69]

$$
\rho T \frac{D s}{D t}=\sigma \nabla \mathbf{u}-\nabla \amalg
$$


siendo la expresión de la entropía $s=c_{p} \ln \left(p^{1 / \gamma} / \rho\right)$, donde $\gamma$ es el cociente de los calores específicos. Empleando la notación tensorial de estas ecuaciones en coordenadas cartesianas $\left(\left\{x_{1}, x_{2}, x_{3}\right\}=\{\mathrm{x}, \mathrm{y}, \mathrm{z}\} ; \mathbf{u}=\left\{u_{i}\right\}_{i=1,2,3}\right.$; $\sigma=\left\{\sigma_{i j}\right\}_{i, j=1,2,3} ; \amalg=\left\{\amalg_{i}\right\}_{i=1,2,3}$ y $\left.D / D t \equiv \partial / \partial t+u_{i} \cdot \partial / \partial x_{i}\right)$, esta ecuación de conservación de la energía (A.15) se rescribe como

$$
\rho T \frac{D s}{D t}=\sigma_{i j} \frac{\partial u_{i}}{\partial x_{j}}-\frac{\partial \amalg_{i}}{\partial x_{i}}
$$

De acuerdo con el cambio de variable introducido por autores como Berman [14] y Goldstein [69], $\eta \equiv \frac{1}{\gamma} \ln \left(p / p_{o}\right)$, se puede obtener una forma más compacta de la teoría de Lilley, reagrupando las ecuaciones (A.12, A.13 y A.16) en una ecuación de tercer orden:

$$
\frac{D}{D t}\left[\frac{D^{2} \eta}{D t^{2}}-\frac{\partial}{\partial x_{i}} a_{o}^{2} \frac{\partial \eta}{\partial x_{i}}\right]+2 \frac{\partial u_{j}}{\partial x_{i}} \frac{\partial}{\partial x_{j}} a_{o}^{2} \frac{\partial \eta}{\partial x_{i}}=q
$$

donde $a_{o}^{2}=\gamma R T$ es la velocidad del sonido, siendo $R$ la constante del gas y la expresión de $q$ representa el término fuente, el cual es interpretado de diferentes formas por diferentes autores. Así, por ejemplo Doak [54] considera que

$$
q=-\frac{\partial u_{i}}{\partial x_{j}} \frac{\partial u_{j}}{\partial x_{k}} \frac{\partial u_{k}}{\partial x_{i}}
$$

para el caso de un flujo ideal, no viscoso, donde no exista conducción de calor a través de él. A este respecto Goldstein [69] asume una formulación más general considerando la siguiente expresión de este término fuente:

$$
q=-\frac{\partial u_{i}}{\partial x_{j}} \frac{\partial u_{j}}{\partial x_{k}} \frac{\partial u_{k}}{\partial x_{i}}+\chi
$$

donde $\chi$ representa los efectos de las fluctuaciones de entropía y viscosidad del fluido.

Estas ecuaciones de Lilley adoptan formas más simplificadas si se particulariza, por ejemplo, si despreciamos los efectos de viscosidad y conducción de calor (aproximación válida en gran variedad de casos), asumiendo la presencia de un flujo medio transversal y unidireccional, y linealizando la forma del campo acústico $\eta$ admitiendo fluctuaciones de presión pequeñas $p^{\prime}=p-p_{o} \ll p_{o}$, del siguiente modo

$$
\eta \equiv \frac{1}{\gamma} \ln \left(1+\frac{p^{\prime}}{p_{o}}\right) \simeq \frac{1}{\gamma} \frac{p^{\prime}}{p_{o}}
$$

La ecuación de Lilley (A.17) puede reescribirse [69]de la siguiente forma

$$
L_{o} \eta=\Gamma
$$


llamada ecuación inhomogénea de Pridmore-Brown, donde $L_{o}$ es el operador de Pridmore-Brown dado por:

$$
L_{o} \equiv \frac{D_{o}}{D t}\left[\frac{D_{o}^{2}}{D t^{2}}-\frac{\partial}{\partial x_{i}} a_{o}^{2} \frac{\partial}{\partial x_{i}}\right]+2 \frac{\partial U}{\partial x_{i}} \frac{\partial}{\partial \mathrm{x}} a_{o}^{2} \frac{\partial}{\partial x_{i}}
$$

donde $D_{o} / D t \equiv \partial / \partial t+U \cdot \partial / \partial \mathrm{x}$, es la derivada convectiva basada en la velocidad media del flujo, $U$.

La expresión detallada de $\Gamma$ se encuentra recogida en el trabajo de Colonious et al. [28]; sin embargo, este término fuente es todavía complicado, por lo que otros autores han derivado formas más simplificadas del mismo. En particular, Goldstein [69] obtiene una expresión de este término fuente como suma de un cuadripolo cuyo esfuerzo es cuadrático con la fluctuación de la velocidad y un dipolo cuyo esfuerzo es proporcional a las fluctuaciones de temperatura; esta forma no se ha incluido en el presente apéndice por la laboriosidad de su obtención y los cambios de variable y modificaciones de la ecuación base (A.21) que conlleva.

Finalmente, comentar en relación a la ecuación de Lilley, que así como Lighthill indicó que la base de la analogía acústica es la demostración de que hay una analogía exacta entre las fluctuaciones de presión de cualquier flujo real y aquellas producidas por una fuente cuadripolar, Lilley señala esta igualdad asociada a una fuente cuadripolar y dipolar. Sin embargo, Goldstein [69] con esta nueva expresión derivada deja patente que esta analogía acústica se produce entre las fluctuaciones de $\left(p / p_{o}\right)^{1 / \gamma}$ en un flujo real y las correspondientes fluctuaciones lineales en su cantidad producida por un cuadripolo más una fuente dipolar de temperatura en un gas ideal arbitrario con flujo medio transversal.

En todas las teorías descritas anteriormente es posible introducir los efectos de la convección turbulenta a velocidad $U_{c}$, a través de la teoría de convección de Lighthill-Ffowcs Williams [149].

Finalmente, se debe mencionar que, independientemente de la naturaleza multipolar particular de cualquier fuente, existe una única interpretación natural de la fuente en aeroacústica, independientemente de que una u otra formulación puede ser más conveniente desde un punto de vista formal. En este sentido Legendre [97] apunta, que tanto la representación cuadripolar de la fuente dada por Lighthill como la representación de Ribner de una distribución de fuentes monopolares, no son contradictorias entre sí, ya que en ninguna de ellas existen singularidades dentro del fluido; simplemente, se ha escogido una representación matemática distinta del proceso físico real que acontece. 


\section{Apéndice B}

\section{Métodos de resolución de ecuaciones de ondas}

B.1 Método de Kirchhoff . . . . . . . . . . . . 249

B.2 Ecuaciones de Ffowcs Williams y Hawkings . . . 250

B.3 Variables de semejanza ............ 252 



\section{B.1 Método de Kirchhoff}

El método de Kirchhoff se aplica a una ecuación de ondas inhomogénea obtenida asumiendo que existe una región de propagación de onda lineal, tal que todas las fuentes dentro de una superficie fija, $\Omega_{s}$, pueden ser reemplazadas por una distribución de fuentes equivalentes en esa superficie. Según Lockard [109] para que esta suposición se verifique, la presión acústica de entrada y su derivada temporal normal a la superficie deben estar dentro de la región de flujo lineal, de forma que sean compatibles con la ecuación de ondas.

En este método general de resolución de problemas de ondas, se considera un fluido ideal y estacionario donde la presión en dominio frecuencia satisface la ecuación de ondas general (4.1) en la cual $\partial / \partial t \rightarrow-i \omega$, siendo $\omega$ la frecuencia angular, esto es, satisface la ecuación de Helmholtz inhomogénea dada por la siguiente expresión

$$
\left(\nabla^{2}+k^{2}\right) p(\mathbf{r}, \omega)=-q(\mathbf{r}, \omega)
$$

donde $q(\mathbf{r}, \omega)$ es la transformada de Fourier de $q(\mathbf{r}, t)$.

Se trata pues de obtener la representación de la solución $p(\mathbf{r}, \omega)$ (presión emitida a un punto $\mathbf{r}$ por una fuente emplazada en un punto $\mathbf{r}^{\prime}$ ), por combinación de la ecuación de Helmholtz (B.1) y la ecuación que satisface la función de Green (4.39). Para ello se llevan a cabo las siguientes transformaciones: (i) se reemplaza $\mathbf{r}$ por $\mathbf{r}^{\prime}$ en (B.1) y se multiplica por $G\left(\mathbf{r}, \mathbf{r}^{\prime} ; \omega\right)$, (ii) se sustrae el producto de $(4.39)$ y $p\left(\mathbf{r}^{\prime}, \omega\right)$ y se integra con respecto a $\mathbf{r}^{\prime} \equiv\left(x_{i}^{\prime}\right)_{i=1, . ., 3}$ sobre todo el fluido. Empleando el teorema de la divergencia el resultado puede escribirse como

$$
\begin{aligned}
p(\mathbf{r}, \omega)= & \oint_{\Omega_{s}}\left\{G\left(\mathbf{r}, \mathbf{r}^{\prime} ; \omega\right) \frac{\partial p\left(\mathbf{r}^{\prime}, \omega\right)}{\partial x_{i}^{\prime}}-p\left(\mathbf{r}^{\prime}, \omega\right) \frac{\partial G\left(\mathbf{r}, \mathbf{r}^{\prime} ; \omega\right)}{\partial x_{i}^{\prime}}\right\} n_{j} d \mathcal{S}\left(\mathbf{r}^{\prime}\right)- \\
& -\int G\left(\mathbf{r}, \mathbf{r}^{\prime} ; \omega\right) q\left(\mathbf{r}^{\prime}, \omega\right) d^{3} \mathbf{r}^{\prime}
\end{aligned}
$$

donde $\mathbf{n}$ es vector unitario normal a la superficie $\Omega_{s}$ en la dirección del flujo. La ecuación B.2 es válida para cualquier solución de la función de Green de (4.39), ésta puede evaluarse si $p$ y $\partial p / \partial x_{n}^{\prime}$ son conocidas en $\Omega_{s}$, pero estas cantidades puede que no estén determinadas independientemente. En realidad, según afirma Howe [79], (B.2) es válida para cualquier superficie estacionaria, incluyendo una superficie de control cerrada, dentro de la cual la presión $p$ a la izquierda de (B.2) pueda reemplazarse por cero, ya que la función $\delta$ en (4.39) no tiene contribución a la integral de volumen fuera de $\Omega_{s}$. Los valores de $p$ y $\partial p / \partial x_{n}^{\prime}$ en la superficie de control (y en una superficie sólida) están, por 
tanto, relacionados de tal forma que dentro de $\Omega_{s}$ sus contribuciones cancelan exactamente el sonido incidente en $\Omega_{s}$ desde alguna otra parte del fluido. Una formulación similar a la ecuación de Kirchhoff en el dominio frecuencia puede darse en el dominio del tiempo de la forma

$$
\begin{aligned}
p(\mathbf{r}, t)= & \oint_{\Omega_{s}}\left\{p\left(\mathbf{r}^{\prime}, \tau\right) \frac{\partial G\left(\mathbf{r}, \mathbf{r}^{\prime} ; t-\tau\right)}{\partial x_{i}^{\prime}}-\frac{\partial p\left(\mathbf{r}^{\prime}, \tau\right)}{\partial x_{i}^{\prime}} G\left(\mathbf{r}, \mathbf{r}^{\prime} ; t-\tau\right)\right\} \times n_{j} d \mathcal{S}\left(\mathbf{r}^{\prime}\right) d \tau \\
& +\int G\left(\mathbf{r}, \mathbf{r}^{\prime} ; t-\tau\right) q\left(\mathbf{r}^{\prime}, \tau\right) d^{3} \mathbf{r}^{\prime} d \tau
\end{aligned}
$$

donde el tiempo retardado de integración respecto a $\tau$ está tomado sobre $(-\infty, \infty)$.

La técnica de Kirchhoff se ha extendido a la acústica computacional. Podrían citarse multitud de trabajos en relación a la aplicación de esta técnica, pero quizás uno de los más representativos sea el realizado por Freund et al. [66], en el cual se calcula el campo sonoro radiado al campo lejano a partir de los datos de campo próximo mediante el empleo del método de Kirchhoff.

\section{B.2 Ecuaciones de Ffowcs Williams y Hawkings}

Muchos de los problemas que aparecen en el estudio práctico de la generación sonora por flujo, provienen de la existencia de fuentes interaccionando con sus contornos, o turbulencia en las capas de corte que separan un medio en reposo de flujos de alta velocidad. Para aplicar ecuaciones acústicas como la de Lighthill a estos fenómenos es necesario, según Howe [79], introducir superficies de control $\Omega_{s}$. Estas superficies pueden coincidir con la superficie del sólido en movimiento o marcar una interconexión entre regiones del fluido de propiedades ampliamente diferenciadas, de modo que para la resolución de la ecuación de ondas para la onda saliente, se introduce la función de Heaviside $H$, resultando una ecuación diferencial de la forma

$$
\begin{aligned}
\left(\frac{1}{a_{o}^{2}} \frac{\partial^{2}}{\partial t^{2}}-\nabla^{2}\right)\left[H\left(p-p_{o}\right)\right]= & \frac{\partial^{2}\left(H T_{i j}\right)}{\partial x_{i} \partial x_{j}}-\frac{\partial}{\partial x_{i}}\left(\left[\rho u_{i}\left(u_{j}-\bar{u}_{j}\right)+p_{i j}^{\prime}\right] \frac{\partial H}{\partial x_{j}}\right) \\
& +\frac{\partial}{\partial t}\left(\left[\rho\left(u_{j}-\bar{u}_{j}\right)+\rho_{o} \bar{u}_{j}\right] \frac{\partial H}{\partial x_{j}}\right)
\end{aligned}
$$

que es la forma diferencial de la ecuación de Ffowcs Williams-Hawkings (FW$\mathrm{H})$, donde $p_{i j}^{\prime}=\left(p-p_{o}\right) \delta_{i j}-\sigma_{i j}$ y $\overline{\mathbf{u}}$ es la velocidad en $\Omega_{s}$, ecuación válida en todo el espacio. Empleando las funciones de Green de la onda emergente 
(4.40) esta ecuación (B.4) se transforma y se reescribe en su forma integral como

$$
\begin{aligned}
H\left(p-p_{o}\right)= & \frac{\partial^{2}}{\partial x_{i} \partial x_{j}} \int_{\mathrm{V}} T_{i j} \frac{d^{3} \mathbf{r}^{\prime}}{4 \pi\left|\mathbf{r}-\mathbf{r}^{\prime}\right|}-\frac{\partial}{\partial x_{i}} \oint_{\Omega_{s}}\left[\rho u_{i}\left(u_{j}-\bar{u}_{j}\right)+p_{i j}^{\prime}\right] \frac{d \mathcal{S}_{j}}{4 \pi\left|\mathbf{r}-\mathbf{r}^{\prime}\right|} \\
& +\frac{\partial}{\partial t} \oint_{\Omega_{s}}\left[\rho\left(u_{j}-\bar{u}_{j}\right)+\rho_{o} \bar{u}_{j}\right] \frac{d \mathcal{S}_{j}}{4 \pi\left|\mathbf{r}-\mathbf{r}^{\prime}\right|}
\end{aligned}
$$

Esta ecuación puede ser simplificada si la superficie de control es estacionaria, $\overline{\mathbf{u}}=0$, reduciéndose en este caso a la ecuación de Curle [79]

$$
\begin{aligned}
H\left(p-p_{o}\right)= & \frac{\partial^{2}}{\partial x_{i} \partial x_{j}} \int_{\mathrm{V}} T_{i j} \frac{d^{3} \mathbf{r}^{\prime}}{4 \pi\left|\mathbf{r}-\mathbf{r}^{\prime}\right|}-\frac{\partial}{\partial x_{i}} \oint_{\Omega_{s}}\left[\rho u_{i} u_{j}+p_{i j}^{\prime}\right] \frac{d \mathcal{S}_{j}}{4 \pi\left|\mathbf{r}-\mathbf{r}^{\prime}\right|} \\
& +\frac{\partial}{\partial t} \oint_{\Omega_{s}}\left[\rho u_{j}\right] \frac{d \mathcal{S}_{j}}{4 \pi\left|\mathbf{r}-\mathbf{r}^{\prime}\right|}
\end{aligned}
$$

Si se considera además el caso particular de $\Omega_{s}$ estacionaria y rígida, la expresión B.6 se simplifica a

$$
H\left(p-p_{o}\right)=\frac{\partial^{2}}{\partial x_{i} \partial x_{j}} \int_{\mathrm{V}}\left[T_{i j}\right] \frac{d^{3} \mathbf{r}^{\prime}}{4 \pi\left|\mathbf{r}-\mathbf{r}^{\prime}\right|}-\frac{\partial}{\partial x_{i}} \oint_{\Omega_{s}}\left[p_{i j}^{\prime}\right] \frac{d \mathcal{S}_{j}}{4 \pi\left|\mathbf{r}-\mathbf{r}^{\prime}\right|}
$$

Esta ecuación es una reformulación de la ecuación de continuidad exacta y las ecuaciones de Navier-Stokes.

En este caso, a diferencia del método anterior, tal como comenta Lockard [109], son necesarios los registros temporales de todas las variables del flujo, mientras que no se requieren explícitamente las derivadas espaciales, porque a pesar de que la solución de las ecuaciones de $F W-H$ requiere una integral de superficie y una de volumen, la solución está en general bien aproximada al considerar únicamente la integral de superficie, ya que ésta está asociada a la transferencia de masa y de momento a través de $\Omega_{s}$. Estas integrales de superficie pueden ser interpretadas como distribuciones de fuentes monopolares o dipolares.

Uno de los resultados más importantes con respecto al modelo anterior es que cuando la integral de superficie es no lineal en el campo próximo, la aproximación de $F W-H$ filtra correctamente la parte de la solución que no radia sonido, mientras que el método de Kirchhoff da resultados erróneos. El método de $F W-H$ se ha aplicado, generalmente, para superficies de integración que coinciden con la superficie del cuerpo sólido, pero es también aplicable cuando la superficie está fuera del cuerpo y es permeable. 
Un ejemplo de la potencia de este nuevo método se puede encontrar en el trabajo desarrollado por Lockard [109], donde se obtiene una formulación integral en dos dimensiones de la ecuación de $F W-H$ en dominio frecuencia para fuentes en un movimiento uniforme, subsónico y rectilíneo. En este trabajo se demuestra que este método es viable con datos de entrada de un campo próximo no lineal donde el método de kirchhoff es impracticable.

\section{B.3 Variables de semejanza}

Estas variables son empleadas para derivar una "ley fundamental" para la obtención de una solución de una ecuación de onda convectiva a partir de la solución de una ecuación de onda no convectiva, sin corresponderse esta ley con una transformación Galileana. En este campo, Chapman [24] derivó una familia paramétrica de variables de semejanza para la descripción de la radiación sonora en un flujo uniforme. Estas variables, basadas en las transformaciones de tipo Lorentz entre la ecuación de onda y la ecuación de onda convectiva, contienen factores Doppler y las variables generales de PrandtlGlauert, usadas en aerodinámica. Los parámetros que especifican la familia de variables son escogidos para que correspondan a la dependencia frecuencial del sonido con el número de Mach del problema analizado.

La ventaja de emplear las variables de semejanza es que los resultados adquieren la misma forma funcional que el problema correspondiente sin flujo, y la dependencia del campo acústico con el número de Mach del flujo es descrita e interpretada fácilmente. 


\section{Apéndice $\mathrm{C}$}

\section{Análisis de errores de los sistemas de medida}

C.1 Características de los sensores . . . . . . . 255

C.1.1 Transductores de medida de presión en cánula . . 255

C.1.2 Micrófonos empleados para la medida de presión en conductos . . . . . . . . . . . . . 257

C.1.3 Sondas de intensidad . . . . . . . . . . . . 258

C.1.3.1 Errores de medida . . . . . . . . . . . . 259

C.1.3.2 Influencia de la posición de medida de intensidad . . . . . . . . . . . 261

C.2 Sistemas de calibración . . . . . . . . . 263

C.2.1 Calibración de los transductores de presión . . . . 263

C.2.2 Método de calibración de los micrófonos de medida de presión en conductos . . . . . . . . . . . . 264

C.2.3 Calibración de la sonda de intensidad . . . . . . . 264

C.3 Características de la cámara semianecoica . . . 272 



\section{C.1 Características de los sensores}

\section{C.1.1 Transductores de medida de presión en cánula}

El sistema de medida de presión en cánula se compone de:

- Transductores piezoeléctricos Kistler 7031.

- Cables de carga microdot-BNC KISTler 1631C2.

- Amplificadores de carga Kistler 5011.

- Cables coaxiales.

- Cadena de adquisición de datos YOKOGAWA DL708E.

En el proceso de medida seguido, el transductor convierte la variable mecánica a medir, en nuestro caso presión, en una carga eléctrica proporcional a la variable que se mide. Esta carga eléctrica es transformada en una señal de tensión en los acondicionadores de señal antes de entrar a la cadena de medida que la registra de forma continua y durante un intervalo de tiempo fijado por el usuario. Al final, los niveles de tensión registrados son representativos de las variaciones de presión producidas.

La conexión de los diferentes elementos que componen el sistema de medida se efectúa de forma que podamos obtener una representación de la magnitud que estamos midiendo (presión) lo más realista posible. Para ello ajustaremos la sensibilidad del acondicionador al valor indicado en la hoja de calibración del transductor correspondiente, según el rango de medida adecuado. La señal de tensión de salida del acondicionador se lleva al módulo de entrada correspondiente de la cadena. Definiendo en el programa de adquisición las unidades que vamos a medir y el factor de conversión de la variación de presión representada, podremos adquirir y medir las ondas de presión según la escala previamente seleccionada en el acondicionador.

La necesidad de calibrar el sistema de medida se debe a que los transductores pierden sensibilidad con las horas de trabajo, de tal modo que para seguir teniendo la misma respuesta en el mismo rango de medida, es necesario aumentar la ganancia del amplificador de señal. Por este motivo, la rutina de calibración del sistema de medida, que será descrita en la siguiente sección, se efectúa para calibrar todos los transductores entre sí, asegurándonos de que todos miden igual magnitud de presión. Es necesario mencionar que la calibración del transductor con sus cables, amplificador, etc., implica el no poder 
intercambiar ninguno de estos elementos a la hora de medir, sin recalibrar de nuevo todo el sistema.

Por otro lado, es necesario considerar las características de la señal a medir a la hora de efectuar una correcta calibración del instrumental utilizado. En nuestro caso particular, la señal a medir es la fluctuación de presión en diferentes puntos del sistema de escape, la cual posee las siguientes características:

- Las señales de presión generadas son pseudo-periódicas.

- Las señales incorporan ruidos de tipo aleatorio, por vibraciones, ruido eléctrico del sistema de adquisición, etc.

- La curva de presión presenta zonas con elevados gradientes. El número de muestras para obtener la máxima resolución posible está en relación con la frecuencia de muestreo elegida y la ventana temporal requerida.

Como consecuencia de ello, al medir presiones instantáneas en un sistema de escape real, y debido a los problemas que pueden presentar los transductores por los cambios de temperatura de los gases de escape en los diferentes puntos de funcionamiento del motor, debemos asegurar la estabilidad del sistema antes de empezar a registrar. Para ello, al cambiar de un régimen de giro a otro, dejaremos el tiempo suficiente para que se estabilice la temperatura de los gases de escape y por tanto de los transductores.

Dos características muy importantes a la hora de calibrar y usar los transductores son su sensibilidad $(p C / b a r)$ y su linealidad en el rango de presiones a medir y la temperatura de utilización. Ambas características son recogidas en la hoja de calibración de cada transductor que es suministrada por el fabricante. Por último, merece especial mención el hecho de que la sensibilidad de los transductores varía ligeramente de unos a otros de forma diferente con los cambios de temperatura y rangos de presión. Por ello los transductores están refrigerados mediante un circuito cerrado de agua para estabilizar su funcionamiento en los diferentes regímenes de motor, es decir, con los cambios de temperatura. 


\section{C.1.2 Micrófonos empleados para la medida de presión en con- ductos}

Los micrófonos empleados para la medida en cánula son micrófonos prepolarizados modelo Brüel\&Kjær 4935 de $\frac{1}{4}$ " de diámetro, adecuados para la medida de presión en conducto. Esta medida de presión se realiza de forma simultánea por un array de micrófonos emplazados en la cánula.

Eléctricamente estos transductores son condensadores formados por una placa fija y otra placa móvil que es el diafragma, donde la cámara de aire intermedia ha sido sustituida parcialmente por un polímero llamado electret como se puede ver en la figura C.1. Dicho polímero ha sido prepolarizado durante su fabricación, es decir, se ha inducido en él una carga eléctrica permanente, que será la que suministre la energía electrostática necesaria para mantener el condensador cargado. De forma que cuando el diafragma reciba una onda de presión se deformará, variando el volumen de aire. Esta variación de volumen, o de capacidad, obliga a que la energía eléctrica almacenada también varíe, produciéndose una corriente que circula a través del circuito que se cierra con una resistencia externa de carga. La caída de tensión producida sobre esa carga externa es proporcional a la presión incidente, de esta forma podemos determinar la medida de presión en dicho punto.

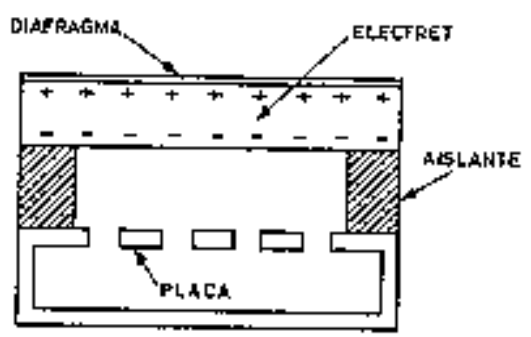

Figura C.1: Esquema del micrófono electrostático de electret

Estos micrófonos poseen un limite de error respecto a la medida del espectro de presión sonora de $\pm 1 \mathrm{~dB}$ en el rango de frecuencias de 100 a 3 $\mathrm{kHz}$ y de $\pm 2 \mathrm{~dB}$ en el rango de $3 \mathrm{kHz}$ a $5 \mathrm{kHz}$. Errores que minimizamos calibrándolos cuidadosamente antes de cada medida, como se detallará en la siguiente sección. 


\section{C.1.3 Sondas de intensidad}

Como ya se mencionó anteriormente, las medidas de ruido exterior se realizan con una sonda de intensidad del tipo 4197 de la firma Brüel \& Kjær, mostrada en la figura C.2. Básicamente esta sonda de intensidad consiste en dos micrófonos enfrentados con un espaciador rígido entre ellos. Esta disposición es adoptada para obtener una mejor respuesta en frecuencia y directividad característica, que en montajes 'lado a lado' o 'back to back' sin espaciador rígido.

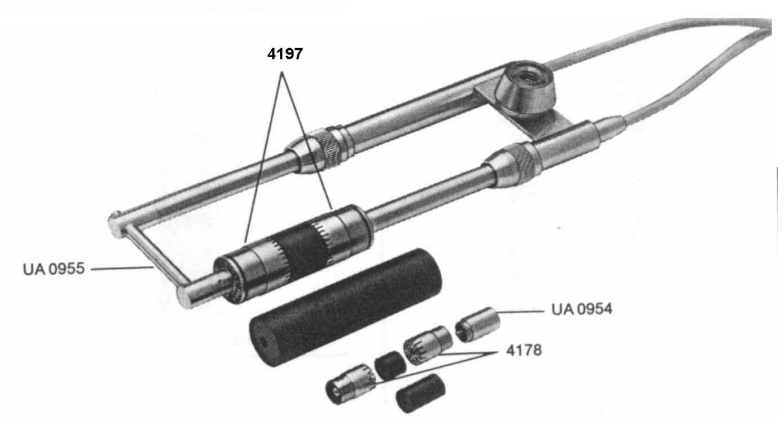

Figura C.2: Sonda de intensidad y accesorios

Tres espaciadores diferentes definen la separación efectiva entre los micrófonos en 6, 12 ó $50 \mathrm{~mm}$, y se escogen en función del rango de frecuencia a cubrir. El tamaño de los micrófonos empleados también depende del contenido frecuencial de la medida. En particular, para nuestro caso se ha escogido un tamaño de $1 / 2$ ", ya que es más adecuado para el rango de frecuencias bajas y medias, a las cuales se encuentran los principales armónicos del motor. Sin embargo para frecuencias más altas y debido a las interferencias que estos pueden producir deben de sustituirse por micrófonos de 1/4".

Las sondas de intensidad empleadas incluyen una pareja de micrófonos de campo libre modelo 4197, para medidas en rangos de frecuencia bajas y medias. Estas sondas poseen cables separados, que terminan en un conector de 7 pines diseñados para la conexión directa a analizadores de doble canal. Estas sondas, además de las ventajas mencionadas anteriormente, poseen una gran ligereza, lo cuál permite su fácil y preciso posicionamiento en la zona de medida mediante la ayuda de una varilla extensible.

Dado que los micrófonos con que está equipada la sonda son los elementos sensibles a las variaciones de presión, a partir de las cuales conoceremos la 
intensidad sonora en el punto de medida, nos detendremos un instante para realizar una descripción de los mismos a fin de establecer sus limitaciones.

Aunque existen micrófonos con principios de funcionamiento muy variados, para medidas de una cierta precisión se utilizan exclusivamente micrófonos de condensador pues, además de su estabilidad, poseen una respuesta lineal en función de la frecuencia.

Estos micrófonos consisten en una pequeña membrana flexible que se deforma por las variaciones de presión sonora y que constituye la parte móvil de un sensor capacitivo. El volumen existente detrás de la membrana está comunicado con la atmósfera a través de un pequeño orificio con el fin de evitar deformaciones debidas a las variaciones de la presión ambiental. Este orificio produce, además, un amortiguamiento viscoso del movimiento de la membrana. Esta membrana y el electrodo posterior están conectados a una tensión continua de polarización, produciendo una carga eléctrica proporcional a la capacidad $C$ del condensador $(C=$ carga/Voltaje $)$.

Existen algunos micrófonos, generalmente para utilización en equipos portátiles, que evitan la necesidad de una tensión de polarización ya que recubren la membrana o el electrodo posterior, con una fina capa de un material prepolarizado (Electret).

Además de las características geométricas de los micrófonos hemos de tener presente que la presencia del micrófono provoca en sus inmediaciones una difracción de las ondas sonoras, con lo que las medidas obtenidas diferirán de los valores realmente existentes en una magnitud denominada 'corrección para campo libre', que depende de la frecuencia y del ángulo de incidencia de las ondas de presión sobre la membrana, siendo mínima para la incidencia perpendicular de las ondas.

Esta distorsión es más importante a altas frecuencias, cuando la longitud de onda es comparable con las dimensiones del micrófono, tal que para una misma frecuencia la corrección será mayor para micrófonos de mayor diámetro.

\section{C.1.3.1 Errores de medida}

Las medidas de ruido realizadas poseen un determinado error, aunque cabe destacar que este error es de pequeña magnitud y perfectamente medible. Dentro del cálculo del error de medida cometido, es importante hacer referencia no sólo a su valor máximo, sino también, al rango frecuencial para el que es válido. A fin de analizar en profundidad las diferentes fuentes de error existentes en la sonda de intensidad, a continuación se realizará un estudio que se dividirá en errores de baja y de alta frecuencia. 
Existen diversas fuentes de error a alta frecuencia, entre ellas el efecto del espaciador utilizado, cuya elección depende del rango de frecuencia a cubrir. La elección de un espaciador u otro afecta a la aproximación lineal realizada de la tangente a la curva de presión existente entre los dos micrófonos tal como se muestra en la figura C.3.

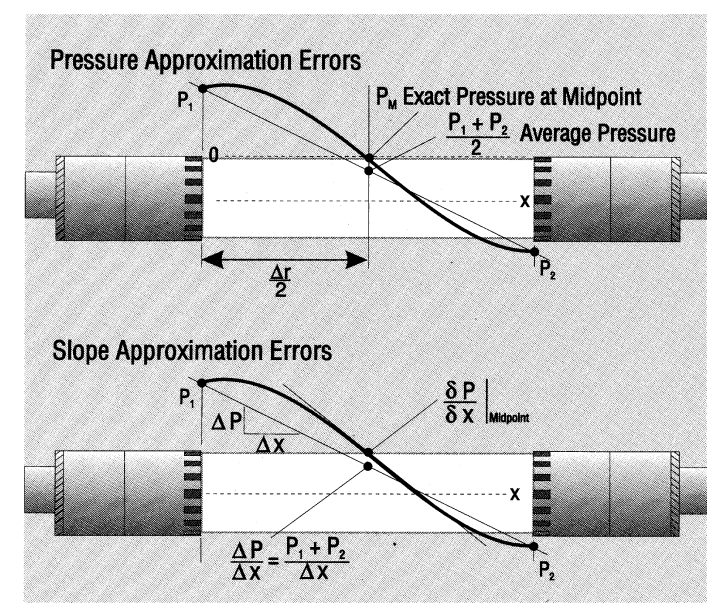

Figura C.3: Error de aproximación de la tangente

Esta aproximación lineal introduce dos errores a alta frecuencia: errores en la medida de presión y errores en la medida de velocidad; errores que se trasladan a la intensidad inferida $(I=p \mathbf{u})$ por la sonda en el punto medio. La intensidad en el punto medio de ambos micrófonos, derivada de las medidas de presión, es comparada con la intensidad exacta en ese punto derivada de la ecuación de la onda de presión acústica exacta. De esta forma, se calcula el error cometido de acuerdo a las especificaciones del fabricante [21], el cuál resulta dependiente del tamaño del espaciador entre los micrófonos y de la longitud de onda. Este error queda cuantificado por la siguiente expresión:

$$
e_{\text {sonda }}=10 \cdot \log \left(\frac{\sin \left(2 \pi \frac{\Delta r}{\lambda}\right)}{2 \pi \frac{\Delta r}{\lambda}}\right)
$$

donde $\Delta r$ representa la separación entre los micrófonos de la sonda como se puede apreciar en la figura C.3.

Esta formula de error es empleada para determinar el rango de frecuencia permisible para un espaciado entre los micrófono determinado. En nuestro caso se optó por tomar un espaciador de $50 \mathrm{~mm}$, lo cual permite hacer predicciones hasta $1 \mathrm{kHz}$, con límites máximos de error de $\pm 1 d B$. 
A baja frecuencia, aparece otra fuente de error, el error de fase entre los micrófonos, que tiene un impacto directo sobre la medida precisa de la velocidad de la partícula. El error asociado a esta diferencia de fase es obtenido a través de la expresión:

$$
e_{\phi}=10 \cdot \log \left(1 \pm 10\left[\delta^{\left[\delta_{p I}-\delta_{p I o}\right] / 10}\right)\right.
$$

donde $\delta_{p I}$ y $\delta_{p I o}$ representan el índice de presión-intensidad y de intensidad residual respectivamente. Magnitudes cuya definición y obtención se estudiará con mayor profundidad en el apartado C.II.3, haciendo mención aquí, únicamente al hecho de su asociación con la extensión direccional en la sonda, es decir, la separación entre los micrófonos de la misma y el desfase que exista entre las señales registradas por ambos.

Por otro lado, el diámetro del captador utilizado es igualmente importante a la hora de determinar el rango de validez de los resultados. Los diámetros de los micrófonos están normalizados a valores de 1", 1/2", 1/4" y 1/8", utilizándose los de mayor diámetro para medidas de niveles de presión sonora a bajas frecuencias, y los de menor diámetro para medidas a frecuencias elevadas. En nuestro caso particular, se escogen los de 1/2" de diámetro, ya que cubren un rango de frecuencias entre $20 \mathrm{~Hz}$ y $6.3 \mathrm{kHz}$.

De esta forma, y atendiendo a todos los comentarios anteriores, el límite de validez de las medidas quedará comprendido dentro del rango de $20 \mathrm{~Hz}$ y 1 $\mathrm{kHz}$, a condición de que el límite de error en baja frecuencia establecido por el error de fase sea inferior o igual al mencionado.

Finalmente, para poder realizar una medición correcta de la intensidad acústica, y una vez analizados los rangos de validez de las mismas, se hace necesaria la calibración del sistema a utilizar.

\section{C.1.3.2 Influencia de la posición de medida de intensidad}

Cuando medimos potencia sonora, la sonda deberá ser posicionada perpendicular a la superficie de medida, para evitar errores, tal como muestra la figura C.4. En ella se observa que si el eje de la sonda está desviado $10^{\circ}$, la medida de intensidad tendrá un error de tan solo $0.07 \mathrm{~dB}$; pero si está desviado $90^{\circ}$, el error inválida completamente las medidas de potencia sonora. Esto significa que para localizar una fuente de ruido de forma precisa usando intensimetría la posición de la sonda es determinante.

Para tratar de minimizar este factor, se sitúa el extremo abierto de la cánula de salida enrasado con un panel perpendicular a dicha sección, el 
cual actúa como superficie reflectante. De esta forma se asegura una mejor direccionalidad del flujo y disponer de una fuente "puntual".

Puesto que la presión es una magnitud escalar, un transductor de presión debería tener igual respuesta para cualquier dirección de incidencia sonora, pero por el contrario, la intensidad sonora es una magnitud vectorial, de modo que el uso de una sonda con dos micrófonos permite medir la componente de este vector en la dirección del eje de la sonda.

El vector intensidad se obtiene a partir de tres componentes perpendiculares entre sí. Para incidencia $90^{\circ}$ respecto al eje, se obtendrá un valor nulo de intensidad y velocidad de partículas. Para un dirección de incidencia arbitraria $\theta$, la intensidad a lo largo del eje será estimada como $I_{\text {medida }}=I \cdot \cos \theta$, lo cuál confiere un patrón de directividad similar al de la función coseno, (figura C.4). Por tanto las características de directividad para el sistema analizador de intensidad sonora aparece, en dos dimensiones, como una figura en forma de ocho (ver figura C.4). Esta relación del coseno implica que el error cometido cuando se realiza una medida a $\theta$ grados tenga la forma: $I_{\theta} / I_{0^{\circ}}=10 \cdot \log (\cos \theta)$.

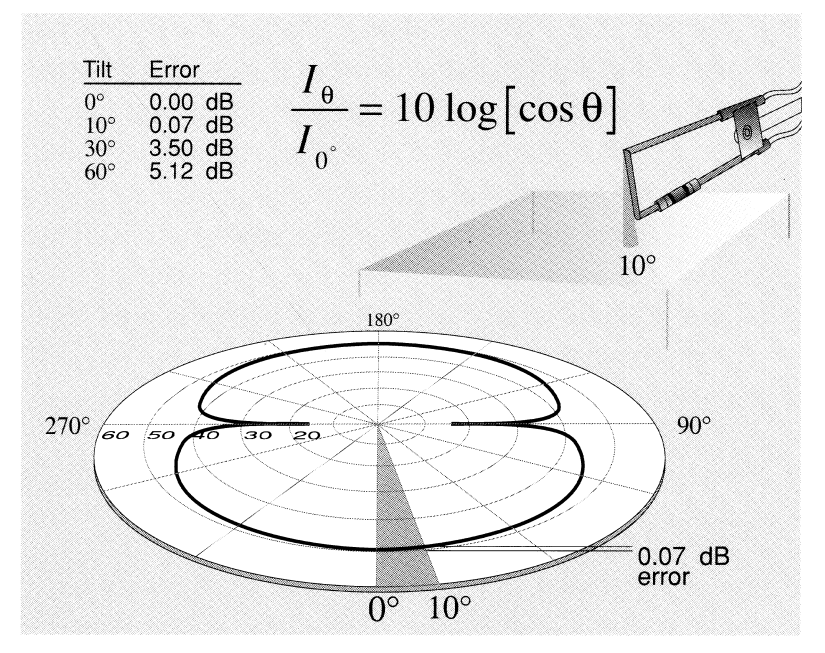

Figura C.4: Diagrama de recepción de la sonda y errores asociados al desfase angular cometido

Además de todo ello, todas las hipótesis empleadas en el modelo corresponden a condiciones de campo libre, por tanto la coherencia de los resultados dependerá de la distancia relativa entre la fuente y el punto de medida, de manera que para posiciones más alejadas del extremo abierto de la cánula los resultados serán presumiblemente mejores puesto que la aproximación a campo libre tendrá mayor validez. 


\section{C.2 Sistemas de calibración}

\section{C.2.1 Calibración de los transductores de presión}

Para comprobar que los transductores de medida de presión en cánula están bien calibrados, es decir, que todos miden una misma señal de presión de la misma manera, se montan en un tubo preparado especialmente para la calibración. Situando los cuatro transductores en el mismo plano, lo más enrasados posible con la superficie interior del tubo y efectuando adquisiciones simultáneas de la misma perturbación de presión, se pueden comparar directamente los registros de presión. El tubo utilizado está preparado para calibrar cuatro transductores a la vez, tal como se observa en la figura C.5.

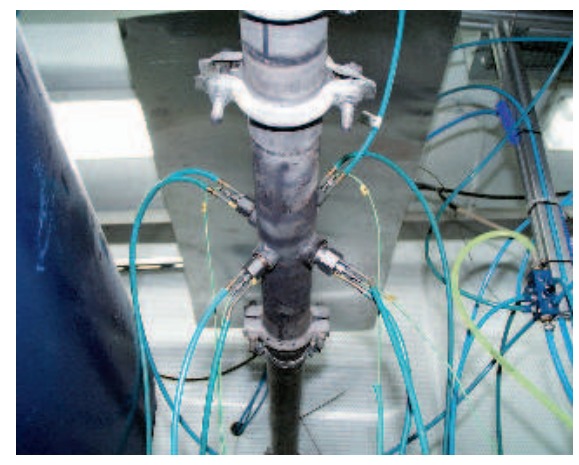

Figura C.5: Tubo de calibración de los transductores

Una vez emplazados los transductores en el tubo se visualiza en la cadena de medida las señales registradas, comprobándose que los pulsos registrados tengan semejanza tanto en amplitud como en duración (período). Si al comparar los registros realizados, se observan diferencias importantes entre las señales registradas en el dominio tiempo se ajusta en el amplificador la sensibilidad del transductor correspondiente, con el fin de tener ondas con amplitud y duración semejantes. Durante este proceso, también se realiza el ajuste del nivel de cero de todos los registros.

Una consideración adicional, muy importante, es aquella que relaciona el nivel espectral del pulso de presión generado y el umbral característico del sistema de adquisición a emplear. Lógicamente, al hablar de sistema de adquisición se está considerando todo el conjunto de elementos que transforman la magnitud física presión en una señal digital. 


\section{C.2.2 Método de calibración de los micrófonos de medida de presión en conductos}

Para poder llevar a cabo las medidas de presión sonora se hace necesaria la calibración del sistema a utilizar, calibrándose estos sensores antes de cada ensayo. Para ello, se hace uso de un pistófono tipo 4228 de Brüel \& Kjær unido al adaptador DP0775 necesario para poder acoplar el micrófono al pistófono como se observa en la figura C.6. La calibración de cada micrófono se hace por separado y a cada uno de ellos le corresponde un canal de entrada en el módulo de adquisición de datos.

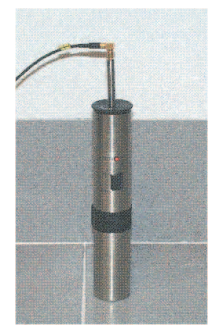

Figura C.6: Calibración del micrófono de medida de presión en cánula

El modo de calibración se basa en que el pistófono genera una señal sonora bien definida (124.06 dB a una frecuencia de $251 \mathrm{~Hz}$ ) similar a las existentes en un campo libre y que es proporcionado por su hoja de calibración. De esta forma, se calibra la relación sensibilidad-intensidad, incorporando todos los componentes, desde los micrófonos hasta las unidades de presentación de resultados. Para una correcta calibración, el valor teórico de la intensidad correspondiente a la fuente (pistófono) $124.06 \mathrm{~dB}$ debe corregirse con el factor de variación de la presión ambiental que es medida con el barómetro corrector, y cuyo valor es añadido al valor teórico proporcionado por las hojas de calibración.

\section{C.2.3 Calibración de la sonda de intensidad}

Para las medidas exactas de intensidad sonora, las medidas de presión de los dos micrófonos deben estar bien emparejadas en fase. En esta sección y de acuerdo con las normas del fabricante [20] se describe el uso del calibrador de intensidad sonora de tipo 3541 de Brüel \& Kjær, empleado en la calibración del sistema analizador de intensidad completo, así como la corrección de la inevitable desigualdad de fase residual entre los dos canales. 
El calibrador de la intensidad sonora 3541 lo constituyen un pistófono tipo 4220, un acoplador de intensidad UA 0914 y una fuente sonora ZI 0055, que pueden verse en la figura C.7. El calibrador tiene dos modos de operación. En el primer modo se genera una señal sonora bien definida, similar a las existentes en un campo libre. Este modo se utiliza para la calibración de la relación sensibilidad-intensidad, incorporando todos los componentes desde los micrófonos a las unidades de presentación de los resultados. El segundo modo de operación se utiliza para la medida del indice de presión-intensidad residual del sistema. Este índice es una medida de la desviación de fase entre los canales o de la capacidad del sistema para reproducir resultados válidos cuando las medidas son efectuadas en campo libre. Este sistema de calibración puede incluso utilizar el índice medido para mejorar la precisión de las medidas por corrección de los resultados de intensidad medida en campo libre.

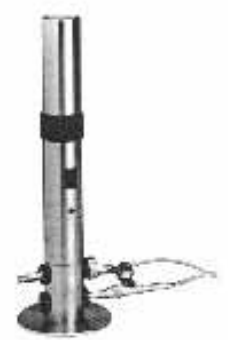

Figura C.7: Dispositivo para la calibración de la amplitud de presión

Existen otros sistemas de calibración de intensidad generando ondas en campo libre, en cámaras anecoicas y ondas estacionarias en el interior de conductos. Estos métodos están diseñados para usos en el laboratorio, ya que debido a la complejidad que supone crear un campo libre bien definido incluso en cámaras anecoicas, u ondas estacionarias en tubos con el equipo adicional necesario, no son prácticos, ya que supondría trasladar, en general, el actual lugar de medida.

El acoplador de intensidad (UA0941), ver figura C.8, reduce extraordinariamente la sensibilidad de la abertura de los micrófonos, por tanto sólo sus diafragmas necesitan ser expuestos al campo sonoro en el acoplador. Esto permite que el acoplador sea de pequeñas dimensiones, lo que implica además un amplio rango de frecuencia de operación. 


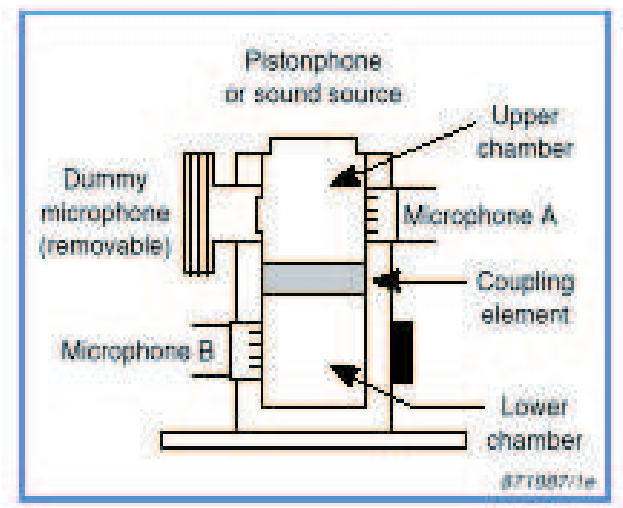

Figura C.8: Sección transversal del acoplador de intensidad

En resumen una calibración de todo el sistema contempla:

1. Fijar las condiciones del medio: densidad, $\rho_{o}$, temperatura $T_{o}$, presión $p_{o}$.

2. Fijar el espaciado entre los transductores, $\Delta r$ (espaciador rígido).

3. Emparejamiento de la fase entre los canales.

4. Sensibilidad de la medida de intensidad sonora.

5. Sensibilidad de la medida de presión sonora.

Las medidas mencionadas en los puntos del 3 al 5 serán realizadas a través del uso del calibrador 3541.

La importancia de determinar las condiciones ambientales y del espaciador, radica en el hecho de que se obtienen los factores de corrección apropiados y se configura el sistema de adquisición del intensímetro en base a ellas. Utilizando los valores standard: temperatura ambiente $20{ }^{\circ} \mathrm{C}$, presión atmosférica $101.3 \mathrm{kPa}$, espaciador $50 \mathrm{~mm}$, si alguno de las condiciones ambientales o del espaciador difiere de las anteriores, los términos de corrección son:

- Si la temperatura es distinta de $20^{\circ} \mathrm{C}$, el factor de corrección para el nivel de intensidad acústica a introducir es: $0.024 \mathrm{~dB} /{ }^{\circ} \mathrm{C} \times(T-20){ }^{\circ} \mathrm{C}$. 
- Si la presión ambiental no es de $101.3 \mathrm{kPa}$, el término de corrección para el nivel de presión sonora indicado viene dado directamente en el barómetro corrector incluido en el equipo de calibración.

- Si el espaciador entre los micrófonos es distinto de $50 \mathrm{~mm}$, es necesario modificar con los niveles siguientes:

$\begin{array}{ccc}\text { Espaciador } & \text { Presión sonora } & \text { Intensidad sonora } \\ 12 \mathrm{~mm} & 0 d B & +6.2 d B \\ 6 \mathrm{~mm} & 0 d B & +9.2 d B\end{array}$

Para calibrar la sonda de intensidad se insertan ambos micrófonos en la cámara superior del acoplador (figura C.8) a la cual se adhiere el pistófono que constituye la fuente sonora de frecuencia y amplitud conocida. Mediante esta disposición el analizador puede realizar la calibración de amplitud de la presión para ambos canales simultáneamente.

Cuando insertamos los micrófonos en diferentes cámaras del acoplador de intensidad, ver figura C.8, y conectamos el pistófono o la fuente de ruido, aparecerá una diferencia de fase entre las presiones sonoras de los micrófonos correspondiente al espaciado nominal de $50 \mathrm{~mm}$ y debida a la disposición escogida en el acoplador. Esta configuración, observada en la figura C.8, simula en el interior del acoplador una onda de sonido plana propagándose en un entorno libre de reflexiones, lo cual nos permite calibrar la sensibilidad de los micrófonos y hallar la velocidad de las partículas en sondas equipadas con micrófonos sensibles a presiones similares a las reales.

El nivel de presión sonora producido por el pistófono dentro del acoplador es aproximadamente $118 d B$ en lugar de los $124 d B$ usuales producidos cuando el pistófono se usa directamente con un sólo micrófono. Este efecto resulta de la duplicación del volumen existente delante de los pistones, reduciendo así el nivel de presión sonora en $6 \mathrm{~dB}$. El valor exacto se da en el mapa de la calibración proporcionado con cada tipo de calibrador 3541. Según esta hoja de calibración, el pistófono empleado genera un nivel constante de 117.9 $\mathrm{dB}$, cuando se junta con el acoplador de intensidad, valor que debe alcanzarse a una frecuencia de $251 \mathrm{~Hz}$ con una desviación máxima de $\pm 0.25 \mathrm{~dB}$. A este valor se deben añadir los factores de corrección mencionados anteriormente.

Respecto al ajuste de fase, debe tenerse en cuenta que incluso con los micrófonos emparejados en fase y un analizador basado en técnicas digitales, siempre existirá un desajuste de fase residual en el sistema práctico. El desajuste de la fase debido al analizador solo puede medirse por medio de la rutina 
incluida en el mismo. En esta prueba, una señal de ruido de banda ancha alimenta ambos canales directamente y se mide el espectro cruzado. La medida de esta desigualdad de la fase se representa junto a la máxima desigualdad de fase especificada para el analizador en un formato especial de 'curva de tolerancia'.

En la figura C.9, se muestra cómo pequeñas diferencias en la fase de la respuesta de los micrófonos y los canales de entrada derivan en la detección de 'intensidad residual', parámetro cuyo espectro no es un valor fijo; sino que este aumenta y disminuye, con la medida del nivel de presión sonora; valor que debe tenerse en cuenta cuando interpretamos los datos de intensidad medidos.

Para medir la desigualdad de fase del sistema completo con ambos micrófonos y, por tanto poder determinar la medida del indice de presiónintensidad residual, $\delta_{p I o}$, los micrófonos son emplazados en la cámara superior del acoplador (ver figura C.8), del mismo modo que para la calibración de la amplitud de presión, salvo que ahora en lugar del pistófono se emplea la fuente sonora de banda ancha ZI 0055. La fuente de ruido está controlada por la salida de un generador de señal pseudo aleatorio, que proporciona una señal de ruido blanco. Esta señal de ruido blanco, espectro plano con ancho de banda constante, generada por la fuente de ruido es pasada por un filtro que atenúa $3 \mathrm{~dB}$ por octava para producir ruido rosa, espectro plano con un porcentaje de ancho de banda constante. De este modo, el espectro de presión sonora (en la banda de octavas) medido en el acoplador corresponde a una señal de ruido rosa, que es constante sobre un amplio rango de frecuencia. Ambos micrófonos están expuestos a la misma presión sonora, por lo que no debe existir ningún gradiente de fase entre las dos señales (no habrá ninguna intensidad), de forma que cualquier intensidad detectada es una intensidad residual.

La diferencia entre el nivel de presión medida y el nivel de intensidad residual detectado se conoce como índice de presión-intensidad residual y es designado por $\delta_{p I o}$ en la norma ISO/DP 9614. Este índice expresa la desigualdad de fase residual y es constante para un sistema de medida y una frecuencia dada.

El espectro del índice de presión-intensidad residual se mide con la disposición comentada sustrayendo el espectro de intensidad detectado al espectro de presión sonora (ver figura C.10). 

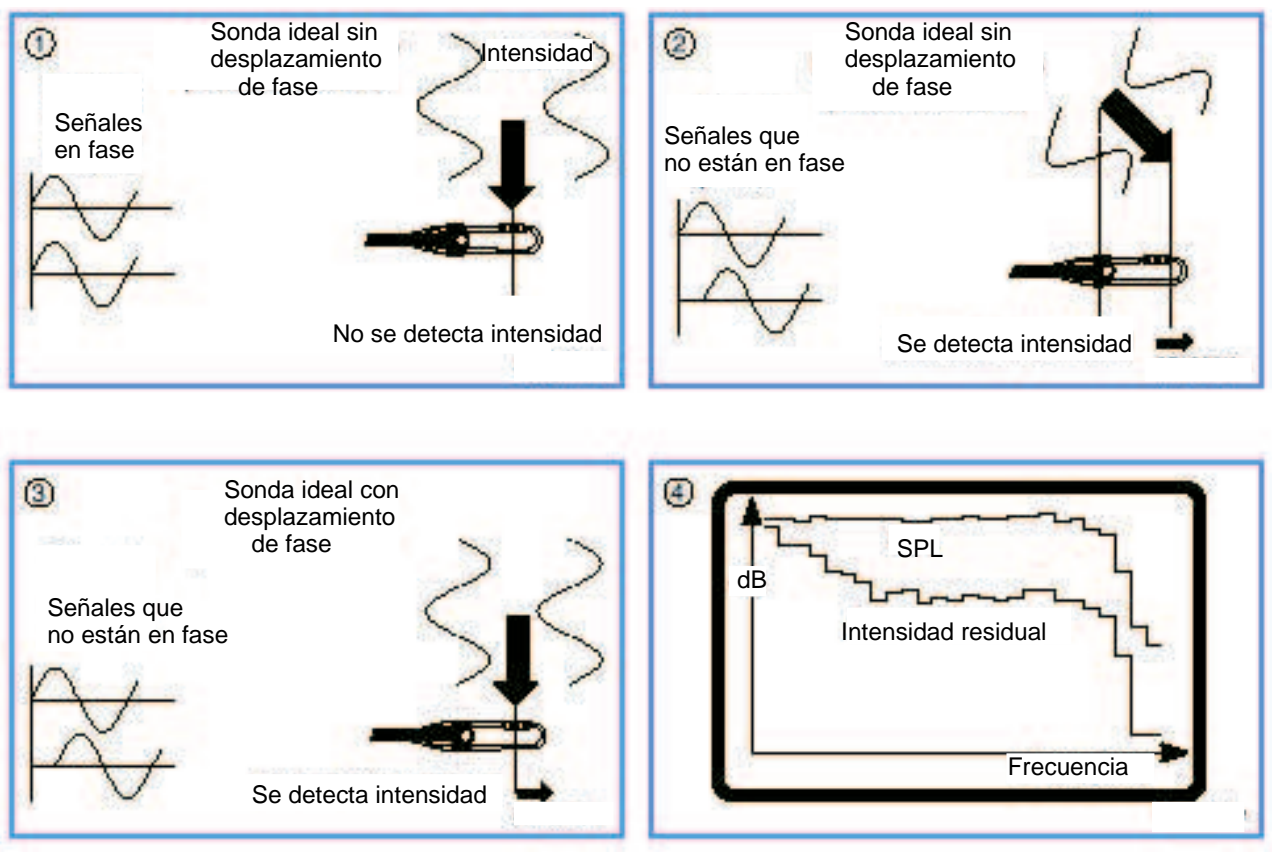

Figura C.9: Intensidad residual, debida al error de fase de los micrófonos: (1) Una onda sonora está incidiendo sobre el eje de la sonda a 90. No hay flujo de energía acústica a lo largo del eje de la sonda. Las señales de los micrófonos están en fase y no existe detección de intensidad. (2) Si la onda sonora incide con un ángulo diferente a 90 , entonces la energía acústica fluye a lo largo del eje de la sonda. Las señales de los micrófonos están fuera de fase y se detecta intensidad. (3) En la práctica si una onda de sonido incide a $90^{\circ}$, pequeñas diferencias entre la fase de los micrófonos causa una pequeña diferencia de fase entre sus señales. No hay manifestación de haber flujo de energía acústica a lo largo del eje de la sonda. (4) Esta presencia de flujo de energía acústica detectada se denomina 'intensidad residual' 


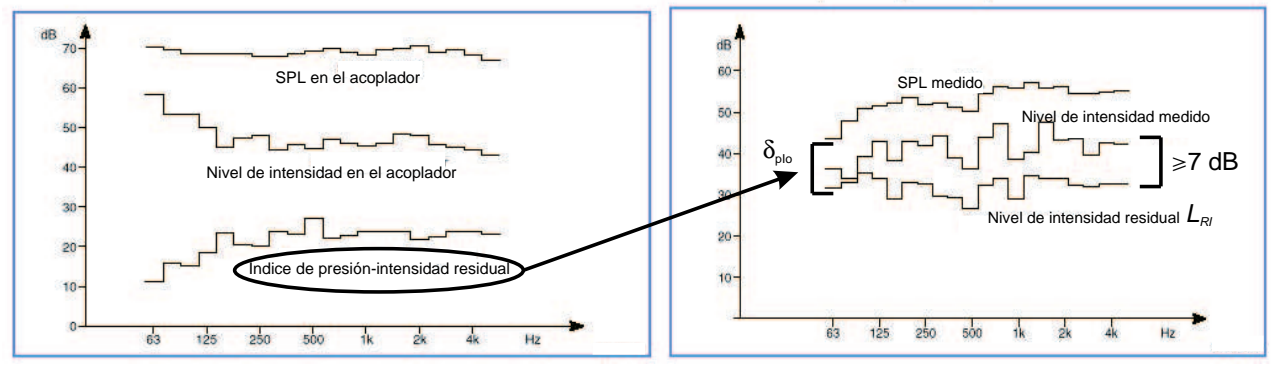

Figura C.10: Calibrado y medida del espectro del $\delta_{\text {pIo }}$ característico del sistema de medida por sustracción de los espectros del nivel de presión e intensidad medidos (izquierda). Obtención del espectro de intensidad residual, $L_{R I}$ (derecha)

Para la determinación de la intensidad y de su espectro medido es necesario conocer la velocidad de la partícula, que se calcula en el punto medio del camino entre ambos micrófonos. La presión sonora en el mismo punto es la media entre las presiones sonoras determinadas por los dos micrófonos, de tal modo que si uno de ellos está en un ambiente muy reactivo, donde pueden tener lugar grandes gradientes de presión, entonces, según las especificaciones del fabricante [20], la intensidad calculada será totalmente incorrecta.

Como se mencionó anteriormente al realizar la medida de intensidad en un entorno completamente reactivo, como un recinto para pruebas de calibración, donde la presión de cada micrófono es la misma y por lo tanto no hay velocidad de partículas, el analizador debería indicar un nivel cero de intensidad. Sin embargo, a causa del error de fase del sistema de medida, existirá un valor de intensidad residual. Esta intensidad residual depende de la frecuencia, de la diferencia de fase del sistema, así como del tamaño del espaciador utilizado. Por esta razón las medidas de intensidad obtenidas $I_{\text {medida }}$, contendrán la intensidad residual del sistema $I_{\text {residual }}$, de modo que la intensidad corregida vendrá dada por

$$
I_{\text {corregida }}=I_{\text {medida }}-I_{\text {residual }}
$$

Expresión que puede reescribirse como:

$$
I_{\text {corregida }}=I_{\text {medida }}-\frac{p_{\text {medida }}^{2} \cdot I_{\text {acoplador }}}{p_{\text {acoplador }}^{2}}
$$

donde $I_{\text {acoplador }}$ y $p_{\text {acoplador }}^{2}$ son la intensidad y la presión obtenidas en el acoplador durante la medida del índice de presión-intensidad residual. 
El índice de presión-intensidad residual $\delta_{p I o}$, se utiliza para evaluar la precisión en la medida de la intensidad sonora. El nivel de intensidad residual $L_{R I}$, se calcula mediante la sustracción del espectro del índice de presión residual $\delta_{p I o}$ al nivel de presión sonora media medido $S P L, L_{R I}=S P L-\delta_{p I o}$, como se muestra en la parte derecha de la figura C.10.

El nivel de intensidad residual se compara posteriormente con la medida del nivel de intensidad sonora, $L_{I}$, a una cierta frecuencia, de forma que el nivel de intensidad residual $L_{R I}$ debe ser al menos $7 \mathrm{~dB}$ menor, para asegurar un error en la medida menor que $1 \mathrm{~dB}$.

El nivel de intensidad residual $L_{R I}$, mostrado en la figura C.10, depende del nivel de presión sonora medido en el campo sonoro y no debe ser confundido con el nivel de intensidad medido con la fuente de ruido rosa.

Otra forma de determinar este límite de error es utilizando el índice de presión-intensidad $\delta_{p I}$, definido como la diferencia entre los niveles de presión e intensidad, el cual es normalmente una cantidad positiva, restringiendose las medidas a los valores de $\delta_{p I}$ que verifiquen: $\delta_{p I} \leq \delta_{p I o}-c_{K}$. Siendo $c_{K}$ una constante escogida de la siguiente manera: si $\mathrm{c}_{K}$ es $7 \mathrm{~dB}$, se puede esperar una precisión de $\pm 1 \mathrm{~dB}$, y si $\mathrm{c}_{K}$ es $10 \mathrm{~dB}$ (como se especifica en la norma ISO 9614), entonces la precisión será $\pm 0.5 d B$.

Este proceso de calibración de la sonda de intensidad empleada debe realizarse antes de cada ensayo a fin de garantizar los límites de validez mencionados. 


\section{C.3 Características de la cámara semianecoica}

Una cámara anecoica es un recinto cuyo campo sonoro interior es libre, es decir, isotrópico, homogéneo y sin superficies límites. Generalmente se logra esta condición revistiendo las paredes interiores con materiales que absorben el sonido (absorbentes sonoros), con lo cual no reflejan el sonido incidente. Estas salas anecoicas están formadas por una estructura aislada del exterior, su interior se acondiciona mediante unas cuñas de longitud a definir en función de la absorción deseada para evitar la reflexión de sonidos por las paredes, suelos y techos. Dichas cuñas se fabrican en diversos materiales como son espuma, fibra de vidrio, lana de roca, etc., presentando el problema de que con el paso del tiempo se descomponen con facilidad desmoronándose y destruyéndose, por lo que constituye un dispositivo de alto coste económico.

La cámara anecoica ideal es un recinto totalmente libre de reverberaciones acústicas, es decir, que cualquier sonido proyectado dentro del recinto, a cualquier frecuencia, es completamente absorbido. En este sentido, es útil construir un recinto tan grande como sea posible, ya que de acuerdo con la ley de emisión con la inversa del cuadrado de la distancia, la energía sonora se disipará de manera que cada metro cuadrado de la superficie interna tendrá menos energía que absorber. Por tanto, un recinto más pequeño requerirá mayor o mejor absorción del sonido para obtener el mismo efecto.

Normalmente las cuñas se construyen del mismo material en la base y la punta (principio de Wedge), con la misma densidad de material en todos los puntos. Es por ello que este tipo de cámaras tiene un buen comportamiento a bajas y medias frecuencias. Para altas frecuencias se emplean las salas Cremer, cuya cuña se va agrandando mientras se acerca a la pared y su densidad se incrementa del mismo modo.

La cámara empleada en las medidas es una cámara semianecioca (una de las paredes es perfectamente reflectante) del tipo Wedge (ver figura C.11). Las dimensiones internas de esta sala son en planta $9.5 \times 9 \mathrm{~m}$ y en altura de $7.2 \mathrm{~m}$ y posee una pared lateral libre de cuñas que realiza la función de reflectante. En ella existe un pasamuros central en el que se enrasa la salida de la cánula de escape procedente de la sala del motor, lo que garantiza simetría en los experimentos, la localización exacta de la fuente sonora, y evita las posibles reflexiones producidas por el tramex del suelo. Todas las paredes y suelo están construidas en hormigón armado formando una jaula que descansa sobre un colchón hidrófugo, de esta forma se consigue aislar toda la estructura de las vibraciones producidas por el ruido externo. Las cuñas empleadas para recubrir el resto de paredes son de fibra de vidrio de una sola pieza y densidad 
constante, de base cuadrada $0.2 \times 0.2 \mathrm{~m}$ y perfil triangular, su longitud total ronda los $0.8 \mathrm{~m}$. Con estas especificaciones se consigue una frecuencia de corte de $100 \mathrm{~Hz}$, por debajo de la cuál se produce una absorción del sonido generado por parte de la cámara inferior al 100\%.

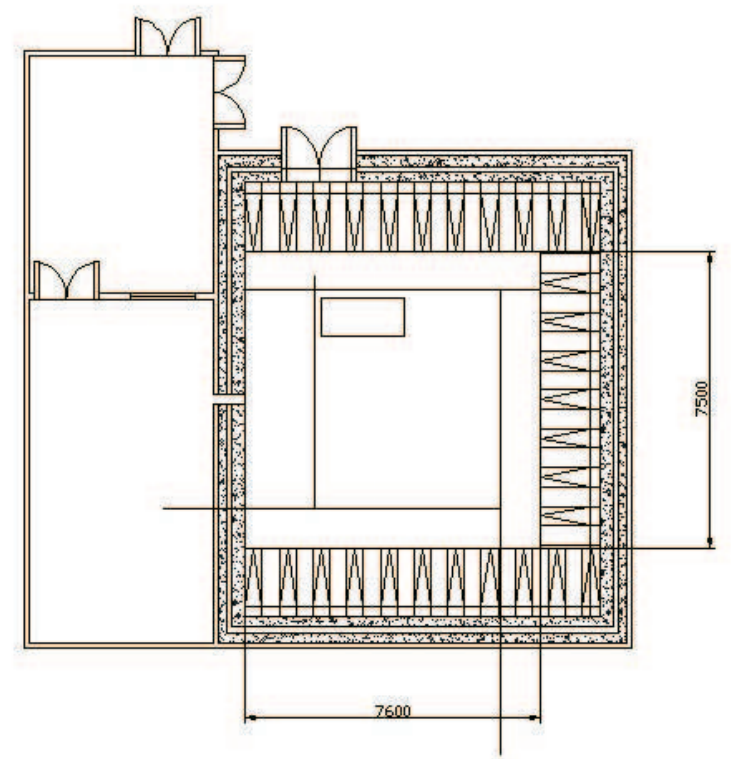

Figura C.11: Planta de la cámara anecoica y salas adjuntas

El suelo tiene una estructura de pilares y perfiles en acero que soportan un tramex permitiendo el acceso del personal a la zona de medida, tramex que es de escaso volumen reduciéndose así la superficie reflectante a fin de que no perturbe las medidas.

Todas estas características de la cámara semianecoica empleada permiten que las medidas sean de la misma calidad que aquellas realizadas en campo libre, ya que no existen reverberaciones que distorsionen el escenario de emisión sonora. 



\section{Bibliografía}

[1] ÅBOM, M. and BODÉN, H. (1988)

Error analysis of two-microphone measurements in ducts with flow. Journal of Acoustical Society of America, 83(6), pp. 2429-2434.

[2] ALFREDSON,R.J. and DAVIES, P.O.A.L. (1970)

The radiation of sound from an engine exhaust.

Journal of Sound and Vibration, 13(4), pp. 389-408.

[3] ANDRéS, I. (2001)

Estudio bibliográfico sobre las bases teóricas de la generación de ruido de flujo.

Trabajo de Investigación 1762/15. Universidad Politécnica de Valencia.

[4] BAILLY, C.; LAFON, P. and CANDEL, S. (1994)

Computation of subsonic and supersonic jet mixing noise using a modified $\mathrm{k}-\varepsilon$ model for compressible free shear flows.

ACTA ACúSTICA, 2 pp.101-112.

[5] BAILly, C.; LAFON, P. and CANDEL, S. (1996)

Computation of noise generation and propagation for free confined turbulent flows.

2nd AIAA/CEAS Aeroacoustics Conference/State College, PA, AIAA 961732, pp. 1-8.

[6] BAILLY, C.; LAFON, P. and CANDEL, S. (1997)

Subsonic and supersonic jet noise predictions from statistical source models.

AIAA Journal, 35(11), pp. 1688-1696.

[7] BANNISTER, F.K.; G.F. MUCKLOW. (1948)

Wave action following sudden release of compressed gas from a cylinder. Proceedings of the Institution of Mechanical Engineers, 159, pp. 269-287. 
[8] BARFORD, N.C. (1985)

Experimental measurements: precision, error and truth (second edition). John Willey \& Sons.

[9] BASTIN, F.; LAFON, P. and CANDEL, S. (1997)

Computation of jet mixing noise due to coherent structures: the plane jet case.

Journal of Fluid Mechanics, 335, pp. 261-304.

[10] BAUMANN, H.D. (1984)

Coefficients and Factors relating to the aerodynamic sound level generated by throttling valves.

Noise Control Engineering Journal, 22(1), pp. 6-11.

[11] BÉCHARA, W.; BAILLY, C.; LAFON, P. and CANDEL, S.M. (1994) Stochastic approach to noise modeling for free turbulent flows.

AIAA Journal, 32(3), pp. 455-463.

[12] BÉCHARA, W.; LAFON, P. and BAILLY, C. (1995)

Application of $\mathrm{k}-\varepsilon$ turbulence model to the prediction of noise for simple and coaxial free jets.

Journal of Acoustical Society of America, 97(6), pp. 3518-3531.

[13] BENAJES, J.; TORREGROSA, A.J.; GALINDO, J. and ANDRéS, I. (2001)

Estimation of the volume velocity fluctuation at the tailpipe end of an I.C. engine exhaust system.

Measurement Sciencie and Technology, 12, pp. 1692-1700.

[14] BERMAN, C.H. (1979)

Some analytical considerations in jet noise prediction.

Mechanics of Sound Generation in Flows, Springer-Verlag, editor: E.A. Müller, pp. 160-165.

[15] BLAIR, G.P., SPECHKO, J.A. (1972).

Sound pressure levels generated by internal combustion engine exhaust systems.

SAE Paper 720155, 563-573.

[16] BLAIR, G.P., COATES S.W. (1973).

Noise produced by unsteady exhaust efflux from an internal combustion engine.

SAE Paper 730160, pp. 657-675. 
[17] BLAIR, G.P. (1990).

The basic design of two-stroke engines. Warrendale, PA.

SAE International.

[18] BODéN, H. and ÅBOM, M. (1986).

Influence of errors on the two-microphone method for measuring acoustic properties in ducts.

Journal of Acoustical Society of America, 79(2), 541-549.

[19] BOGEY, C.; BAILLY, C. and JUVé, D. (2000).

Calcul du rayonnement acoustique d'une couche de mélange à l'aide des équations d'Euler linéarisées.

C. R. Académie des Sciences Paris, t. 328, Série IIb, pp. 341-347.

[20] BRÜEL \& KJÆR (1990).

Sound Intensity calibrator-Type 3541.

Application note.

[21] BRÜEL \& KJÆR (1998).

Sound Intensity Probe Sets.

Brüel \& Kjar Product Data.

[22] CARGILL, A.M. (1982)

Low-frequency acoustic radiation from a jet pipe-A second order theory. Journal of Sound and Vibration, 83(3), pp. 339-354.

[23] CARGILL, A.M. (1982)

Low-frequency sound radiation and generation due to the interaction of unsteady flow with a jet pipe.

Journal of Fluid Mechanics, 121, pp. 59-105.

[24] CHAPMAN, C.J. (2000)

Similarity variables for sound radiation in a uniform flow.

Journal of Sound and Vibration, 233(1), pp. 157-164.

[25] CHEONG, C. and LEE, S. (2001)

Grid-Optimized Dispersion-Relation-Preserving Schemes on General Geometries for Computational Aeroacustics

Journal of Computational Physics, 174, pp. 248-276.

[26] CHUNG, J.Y. and BLASER, D.A. (1980)

Transfer function method of measuring in-duct acoustic properties. I. Theory.

Journal of Acoustical Society of America, 68(3), pp. 907-921. 
[27] CLAVIN, P. and SIGGIA, E.D. (1991)

Turbulent Premixed Flames and Sound Generation.

Combustion Sciencie and Technology, 78, pp.147-155.

[28] COLONIUS, T.; LELE, S.K. and MOIN, P. (1997)

Sound generation in a mixing layer.

Journal of Fluid Mechanics, 330, pp. 375-409.

[29] COMPARIN, R.J. (1991)

Acoustic intensity measurement in the presence of mean flow with application to noise measurements in a wind tunnel.

Journal of Acoustical Society of America, 90 (4), pp.1733-1741.

[30] CRAGGS, A. and AYORINDE, E.O. (1990)

Isochronous oscillators: their importance in low-frequency sound transmission in passenger vehicles

Journal of Sound and Vibration, 142(2), pp. 360-363.

[31] CRIGHTON, D.G. (1979)

Why do the Acoustics and the dynamics of a hypothetical mean flow bear on the issues of soundas generation by turbulence?

Mechanics of Sound Generation in Flows, Springer-Verlag, editor: E.A. Müller, pp. 1-9.

[32] CRIGHTON, D.G. (1981)

Acoustic as a branch of fluid mechanics.

Journal of Fluid Mechanics, 106, pp. 261-298.

[33] CROCKER, M.J. (1997)

General linear acoustics: introduction

Encyclopedia of Acoustics, Wiley-Interscience, editor: M.J. Crocker, 1 pp. 3-19.

[34] CUMMINGS, A. (1981)

Design charts for low frequency acoustic transmission through the walls of rectangular ducts.

Journal of Sound and Vibration, 78(2), pp. 269-289.

[35] DANIELS, V.A.; VERES, R.E. (1976)

The Fourier transform applied to vehicle exterior noise source identification.

SAE Paper 760151. 
[36] DAVIES, P.O.A.L.; BENTO COELHO, J.L.; BHATTACHARYA, M. (1980)

Reflection coefficients for an unflanged pipe with flow.

Journal of Sound and Vibration, 72(4), pp. 543-546.

[37] DAVIES, P.O.A.L. (1981)

Radiation of sound by a hot exhaust.

Journal of Sound and Vibration, 76(4), pp. 591-594.

[38] DAVIES, P.O.A.L. (1981)

Flow-Acoustics coupling in ducts.

Journal of Sound and Vibration, 77(2), pp. 191-209.

[39] DAVIES, P.O.A.L. (1987)

Plane wave reflection at flow intakes.

Journal of Sound and Vibration, 115, pp. 560-564.

[40] DAVIES, P.O.A.L. (1988)

Practical flow duct acoustics.

Journal of Sound and Vibration, 124(1), pp. 91-115.

[41] DAVIES, P.O.A.L. (1988)

Plane acoustic wave propagation in hot gas flows.

Journal of Sound and Vibration, 122(2), pp.389-392.

[42] DAVIES, P.O.A.L. (1989)

Slightly dispersive plane waves in pipes.

Journal of Sound and Vibration, 132(1), pp. 169.

[43] DAVIES, P.O.A.L. (1996)

Aeroacoustics and Time Varying systems.

Journal of Sound and Vibration, 190(3), pp .345-362.

[44] DAVIES, P.O.A.L. (1996)

Piston engine intake and exhaust system design.

Journal of Sound and Vibration, 190(4), pp. 677-712.

[45] DAVIES, P.O.A.L. (1997)

Predictive acoustic modelling applied to the control of intake/exhaust noise of internal combustion engines.

Journal of Sound and Vibration, 202(2), pp. 249-274.

[46] DAVIES, P.O.A.L. and HOLLAND, K.R. (1999)

I.C. Engine intake and exhaust noise assessment.

Journal of Sound and Vibration, 223(3), pp. 425-444. 
[47] DAVIES, P.O.A.L. and HOLLAND, K.R. (2000)

Intake and exhaust system orife noise assessment.

European Conference on Vehicle Noise and Vibration 2000, I.Mech.E. HQ, London, 43-52.

[48] DAVIES, P.O.A.L. and HOLLAND, K.R. (2000)

The measurement of sound power flux in flow ducts

Journal of Sound and Vibration, 230(4), pp. 915-932.

[49] DAVIES, P.O.A.L. and HOLLAND, K.R. (2000)

Intake an exhaust system orifice noise assessment

European Conference on Vehicle Noise and Vibration, IMechE HQ.

[50] DAVIES, P.O.A.L. and Holland, K.R. (2001)

The observed aeroacustic behaviour of some flow-excited expansion chambers.

Journal of Sound and Vibration, 239(4), pp. 695-708.

[51] DAVIES, P.O.A.L.; HOLLAND, K.R. and VAN DER WALT, D.C. (2002) The experimental assessment of flow generated noise emissions from intake and exhaust systems

European Conference on Vehicle Noise and Vibration, IMechE HQ, London, pp. 43-52.

[52] DESANTES, J.M; TORREGROSA, A.J. and BROATCH, A.(2001)

Experiments on flow noise generation in simple exhaust Geometries.

Acustica-Acta Acustica, 87, pp.46-55.

[53] DESANTES, J.M.; TORREGROSA, A.J. and BROATCH, A. (2001)

Wavelet transform applied to Combustion Noise Analysis in High-speed DI Diesel Engines.

SAE Paper 2001-01-1545.

[54] DOAK, P.E. (1979)

A new view of sound generation and propagation in flows.

Mechanics of Sound Generation in Flows, Springer-Verlag, editor: E.A.

Müller, pp. 247-255.

[55] DOAK, P.E. (1992)

Cilindrical wave propagation in a cylindrical diffuser.

Journal of Sound an Vibration. 155(3), pp. 541-544. 
[56] DOAK, P.E. (1998)

Fluctuating Total Enthalpy as the Basic Generalized Acoustic Field.

Theoretical and Computational Fluid Dynamics. Springer-Verlag. 10, pp. 115-133.

[57] DOKUMACI, E. (2000)

On calculation of acoustic power.

Journal of Sound and Vibration, 238(5), pp. 869-876.

[58] DOWLING, A.P. and FFOWCS WILLIAMS, J.E. (1983)

Sound and Sources of Sound.

Chichester: Ellis Horwood.

[59] DOWLING, A.P. (1997)

Steady-State Radiation from Sources.

Encyclopedia of Acoustics, (M.J. Crocker, editor), pp. 107-125. New York: John Wiley \& Sons, Inc.

[60] EL-RAHEB, M. (1981)

Vibrations of three-dimensional pipe systems with acoustic coupling.

Journal of Sound and Vibration, 78(1), pp. 39-67.

[61] EL-SHARKAWY, A.I.; EL-CHAZLY, N.M. (1987)

A critical survey of basic theories used in muffler design and analysis.

Applied Acoustics. 20, pp. 196-218.

[62] FARASSAT, F., BRENTNER, K.S.(1997)

The Acoustic Analogy and the Prediction of the Noise of Rotating Blades. Theoretical and Computational Fluid Dynamics. Springer-Verlag. Abstrat Volume 10 Issues 1-4, pp. 155-170.

[63] FEDORCHENKO, A.T. (2000)

On some fundamental flaws in present aeroacoustic theory.

Journal of Sound and Vibration, 232(4), pp. 719-782.

[64] FFOWCS WILLIAMS, J.E. (1996)

Aeroacoustics.

Journal of Sound and Vibration, 190(3), pp. 387-398.

[65] FILIPPI, P.J.T.; HABAULT, D.; PIRAUX, J. (1988)

Noise sources modelling and intensimetry using antenna measurement and identification procedures.

Journal of Sound and VIbration, 124(2), pp. 285-296. 
[66] FREUND, J.B.; LEE, S.K.; MOIN, P. (1996)

Calculating of the radiated sound field using an open Kirchhoff surface. AIAA Journal, pp. 909-916.

[67] FREUND, J.B. (2001)

Noise sources in a low-Reynolds-number turbulent jet at Mach 0.9.

Journal of Fluid Mechanics, 438, pp. 277-305.

[68] GOLDSTEIN, M.E. and LEIB, S.J. (2000)

Emission of sound from turbulence convected by parallel mean flow in the presence of a confining duct.

Journal of Sound and Vibration, 235(1), pp. 25-42.

[69] GOLDSTEIN, M.E. (2001)

An exact form of Lilley's equation with a velocity quadrupole/temperature dipole source term.

Journal of Fluid Mechanics, 443, pp. 231-236.

[70] GLOERFELT, X.; BAILLY, C. and JUVé, D. (2000)

Calcul direct du rayonnement acoustique d'un écoulement affleurant une cavité.

C. R. Académie des Sciences Paris, t. 328, Série IIb, pp. 625-631.

[71] HARDIN, J.C. and POPE, D.S. (1995)

Sound generation by flow over a two-dimensional cavity.

AIAA Journal, 33(3), pp. 407-412.

[72] HARIHARAN, S.I.; SCOTT, J.R. and KREIDER, K.L. (2000)

A potential-theoretic method for far-fiel sound radiation calculations.

Journal of Computational Physics, 164, pp. 143-164.

[73] HENDERSON, B. and POWELL, A. (1993)

Experiments concerning tones produced by axisymmetric chocked jet impinging on flat plates.

Journal of Sound and Vibration,168(2), pp. 307-326.

[74] HIGASHIYAMA, J. and IWAMOTO, J. (1999).

Experimental study of exhaust noise generated by pulsating flow downstream of pipe end.

JSAE Review 9930018, 20, 73-79.

[75] HOLLAND, K.R.; DAVIES, P.O.A.L. (2000)

The measurement of sound power flux in flow ducts.

Journal of Sound and Vibration, 230(4), pp. 915-932. 
[76] HOWE, M.S. (1975)

Contributions to the theory of aerodynamic noise, with aplications to excess jet engine noise and the theory of the flaute.

Journal of Fluid Mechanics, 71(4), pp. 625-673.

[77] HOWE, M.S; BAUMANN, H.D. (1992)

Noise of gas flow. Beranek L.L. and Ver. I. editors.

Noise and vibration control engineering. Principels and applications, John Wiley and Sons.

[78] HOWE, M.S. (1997)

Edge, cavity and aperture tones at very low Mach numbers.

Journal of Fluid Mechanics, 330, pp. 61-84.

[79] HOWE, M.S. (1998)

Acoustics of Fluid-Structure Interactions.

Cambridge University Press.

[80] HOWE, M.S. (1999)

On Rayleigh's computation of the 'end correction', with application to the compression wave generated by a train entering a tunnel.

Journal of Fluid Mechanics, 385, pp.63-78.

[81] HOWE, M.S. (2000)

Trailing edge noise at low mach numbers, part2: attached and separated edge flows.

Journal of Sound and Vibration, 234(5), pp. 761-775.

[82] HOWE, M.S. (2001)

On the Hydroacoustics of a trailing edge with a detached flap.

Journal of Sound and Vibration, 239(4), pp. 801-417.

[83] JACOBSEN, F. (1990)

Sound Field Indicators: Useful Tools.

Noise Control Engineering Journal, 35(1), pp.37-46.

[84] JACOBSEN, F. (1992)

Sound power determination using the intensity technique in the presence of diffuse background noise.

Journal of Sound and Vibration, 159(2), pp. 353-371.

[85] JEANNEAU, M. and PICHANT, P. (2000)

The trends of steel products in the European automotive industry.

La revue de Métallurgie, pp. 1399-1408. 
[86] JI, Z.L.; MA, Q. and ZHANG, Z.H. (1995)

A boundary element scheme for evaluation of four-pole parameters of duct and mufflers with low mach number non-uniform flow Journal of Sound and Vibration, 185(1), pp. 107-117.

[87] JONES, A.D. (1984)

Modelling the exhaust noise radiated from reciprocating internal combustion engines -A literature review.

Noise Control Engineering Journal, 23 (1), pp. 12-31

[88] KANIA, N. (1985)

Reduction of Primary Noise Originating from a Cranckcase-Scavenged Two-Stroke Engine.

Noise Control Engineering Journal, 28(2), pp. 66-81.

[89] KIM, D.H. and KOSS, L.L. (1990)

Sound radiation from a circular duct with axial temeparture gradients.

Journal of Sound and Vibration, 141(1), pp. 1-16.

[90] KIM, Y.S. and LEE, D.J. (2001)

Numerical analysis of internal combustion engine intake noise with a moving piston and a valve.

Journal of Sound and Vibration, 241(5), pp. 895-912.

[91] KOJIMA, N. LIU, B and ZHOU, H. (1995)

Relation Between the predominance of Acoustic Resonance Noise and Air Flow Muffler

SAE Technical Paper 951262.

[92] KUNZ, F.; GARCIA, P. (1995)

Simulation and Measurements of Hot Exhaust Gas Flow Noise with a Cold Air Flow Bench.

SAE Paper 950546, Society of Automotive Engineers, Warrendale, PA, pp. 63-69.

[93] KUNZ, F.H (1999)

Semi-Empirical Model for Flow Noise Prediction on Intake and Exhaust Systems.

SAE Paper 1999-01-1654, pp. 1-6.

[94] LAMANCUSA, J.S. (1988)

Geometric optimization of internal combustion engine induction systems for minimum noise transmission.

Journal of Sound and Vibration, 127(2), pp. 303-318. 
[95] LANDAU, L.D. E.M. LIFSHITZ (1989)

Mécanique des Fluides.

Deuxième edition, Moscou, Editions Mir.

[96] LEE, D.J. and KOO, S.O. (1995)

Numerical Study of Sound Generation due to a Spinning Vortex Pair.

AIAA Journal, .33, No. 1, pp. 20-26.

[97] LEGENDRE,R. (1992)

High velocity acoustics.

Journal of Sound and Vibration, 157(2), pp. 193-203.

[98] LEIPOLD, F.; BERGMANN, H.(1996)

Development strategies for reducing noise emissions of the new OM 904 LA commercial vehicle diesel engine.

AVL Conference "Engine and Environment" '96, pp. 309-318.

[99] LESIEUR, M.; COMTE, P. and METAIS, O. (1999)

Simulation numérique déterministe de la turbulence.

C. R. Académie des sciences/Elsevier, Paris, t. 327, Série II b, pp. 333341.

[100] LEUNG, R.C.K.; TANG, S.K.; HO, I.C.K. and KO, N.W.M. (1996)

Vortex pairing as a model for jet noise generation.

AIAA Journal, 34(4), pp. 669-675.

[101] LEVINE, H.; SCHWINGER, J. (1948)

On radiation of sound from an unflanged circular pipe.

Physical Review 73, pp. 383-406.

[102] LIGHTHILL, M.J. (1952)

On sound generated aerodynamically: I, general theory.

Proceedings of the Royal Society of London A211, pp. 564-587.

[103] LIGHTHILL, M.J. (1954)

On sound generated aerodynamically: II, turbulence as a source of sound.

Proceedings of the Royal Society of London 222, pp. 1-32.

[104] LIGTHTHILL, M.J. (1978)

Waves in Fluids.

Cambridge, Cambridge University Press. 
[105] LIGHTHILL, M.J. (1998)

Aeroacustic and atmospheric sound: introduction.

Handbook of Acoustics, Wiley-Interscience, editor: M.J. Crocker, pp. 257271.

[106] LILLEY, G.M. (1996)

The radiated noise from isotropic turbulence with applications to the theory of jet noise.

Journal of Sound and Vibration, 190(3), pp. 463-476.

[107] LIN, J.C.; ROCKWELL, D. (1999)

Cinema PIV and its application to Impinging Vortex Systems.

Journal of Fluids Engineering, 121, pp. 720-724.

[108] LO, S.H.; VOKE, P.R. and ROCKLIFF, N.J. (2000)

Three-Dimensional vórtices of a spatially developing plane jet.

International Journal of Fluid Dynamics, 4(1), pp. 1-20.

[109] LOCKARD, D.P. (2000)

An efficient, two-dimensional implementation of the Ffowcs Williams and Hawkings equation.

Journal of Sound and Vibration, 229(4), pp. 897-911.

[110] LOREA, A.; RENZULlO, A.; CHIESA, L. and GUENNA, G. (1999)

Acoustic intensity measurements in exhaust pipes.

EAEC Congress.

[111] MANOJ, K.G. and BHATTACHARYYA, S.K. (2000)

Transient acoustic radiation from impulsively accelerated bodies by the finite element method.

Journal of Acoustical Society of America, 107, pp. 1179-1188.

[112] MARGOT, X. (1993)

A physically guided zonal approach for Euler / Navier-Stokes predictions of aerofoil flows.

PhD Thesis of the University of London.

[113] MAST, T.D and PIERCE, A.D. (1995)

Describing-function theory for flow excitation of resonators.

Journal of Acoustical Society of America, 97(1), pp. 163-172.

[114] MCGOWAN, R.S. (1988)

An aeroacoustic approach to phonation.

Journal of Acoustic Society of America, 83(2), pp. 696-704 
[115] MENGALI, G. and MICHELI, M. (1994)

Simulation of Turbulent Fluctuations.

AIAA Journal, 32, No.11, pp. 2210-2216

[116] MICHALKE, A. (1991)

Sensitivity of a slit-tube probe to higher order acoustic modes in a pipe of circular cross-section.

Journal of Sound and Vibration, 151(1), pp. 157-162

[117] MITCHELL, B. E.; LELE, S. K. and MOIN, P. (1999)

Direct computation of sound generated by vortex pairing in an axisymmetric jet.

Journal of Fluid Mechanics, 383, pp. 113-142.

[118] MÖHRING, W. (1978)

On vortex Sound at low Mach number.

Journal of Fluid Mechanics, 85, part. 4, pp. 685-691.

[119] MONGEAU, L.; THOMPSON, D.E. and MC.LAUGHLIN, D.K. (1995) A method for characterizing aerodynamic sound sources in turbomachines.

Journal of Sound and Vibration, 181(3), 369-389.

[120] MORFEY, C.L. (1971)

Sound transmission and generation in ducts with flow.

Journal of Sound and Vibration, 14(1), pp. 37-55.

[121] MORSE, P.H.; INGARD, K.U. (1986)

Theoretical acoustics.

Princenton, N.J., Princenton University Press.

[122] MOSER, F.X; SPESSER, B. and HALLER, H. (1996)

Possibilities of noise reduction in commercial and industrial diesel engines. AVL Conference "Engine and Environment" '96, pp. 349-366.

[123] MU, S. and MAHALINGAM, S. (1996)

Direct Numerical Simulation of Acoustic/Shear Flow Interactions in TwoDimensional Ducts.

AIAA Journal, 34 (2), pp. 237-243.

[124] MUNJAL, M.L. (1987)

Acoustics of ducts and mufflers.

New York, John Willey \& Sons. 
[125] MUNT, R.M. (1990)

Acoustic transmission properties of a jet pipe with subsonic jet flow: I. The cold jet reflection coefficient.

Journal of Sound and Vibration, 142(3), pp. 413-436.

[126] NASH, E.C.; LOWSON, M.V. and MCALPINE, A. (1999)

Boundary-layer instability noise on aerofoils.

Journal of Fluids Mechanics. 382, pp. 27-61

[127] NELSON, P.A. and MORFEY, C.L. (1981)

Aerodynamic sound production in low speed flow ducts.

Journal of Sound and Vibration, 79(2), pp.263-289.

[128] NORRIS, A.N. and SHENG (1989)

Acoustic radiation from a circular pipe with an infinite infinite flange. Journal of Sound and Vibration 135(1), pp. 85-93.

[129] NUTTAL, A.H.; WILSON, J.H. (1991)

Estimation of the acoustic field directionality by use of planar and volumetric arrays via the Fourier series method and the Fourier integral method.

Journal of the Acoustical Society of America, 90(4), pp. 2004-2019.

[130] OLDHAM, D.J. and UKPOHO, A.U. (1990).

A pressure-based technique for predicting regenerated noise levels in ventilation systems.

Journal of Sound and Vibration 140(2), pp. 259-272.

[131] PAYRI, F; DESANTES, J.M. and TORREGROSA, A.J. (1995)

Acoustic Boundary condition for unsteady one-dimensional flow calculations.

Journal of Sound and Vibration 188(1), pp. 85-110.

[132] PAYRI, F; TORREGROSA, A.J.; CHUST, M.D (1996)

Application of MacCormak schemes to I.C. engine exhaust noise prediction.

Journal of Sound and Vibration 195(5), pp. 757-773.

[133] PAYRI, F.; DESANTES, J.M. and BROATCH, A. (2000)

Modified impulse method for the measurements of the frequency response of acoustic filters to weakly nonlinear transient excitations.

Journal Acoustic Society of America. 107, pp. 731-738. 
[134] PEDERSEN, R.C. and NORTON, M.P. (1997)

Quantification of acoustic and hydrodynamic fields in flow duct systems. Applied Acoustics, 50(3), pp. 205-230.

[135] PEIZI, L. and GENHUA, D. (1991)

Experiment and formulation of pulsed jet noise.

Noise Control and Engineering Journal, 36(1), pp. 33-38.

[136] PEIZI, L.; GENHUA, D. and ZHICHI, Z. (1993)

Noise radiation of strongly pulsating tailpipe exhaust.

Journal of Sound and Vibration, 167(3), pp.385-400.

[137] PETERS, M.C.A.M; HIRSCHBERG, A.; REIJNEN, A.J.; WIJNANDS, A.P. (1993)

Damping and reflection coefficient measurements for an open pipe at low Mach and low Helmholtz numbers.

Journal of Fluid Mechanics, 256, pp. 499-534

[138] PIÑERO, G; VERGARA, L; DESANTES, J.M.; BROATCH, A. (2000) Estimation of velocity fluctuation in the internal combustion engine exhaust system through beamforming techniques.

Measurement Sciencie and Technology, 11, pp. 1585-1595.

[139] POWELL, A. (1964)

Theory of vortex sound.

Journal of the Acoustical Society of America 36(1), pp. 177-195.

[140] POWELL, A. (1995)

Vortex sound: An alternative derivation of Möhring's formulation.

Journal of the Acoustical Society of America 97(1), pp. 684-686.

[141] POWELL, A. (1995)

Vortex sound theory: Direct proof of equivalence of "vortex force" and "vorticity alone" formulations.

Journal of the Acoustical Society of America 97(3), pp. 1534-1537.

[142] POWELL, A. (1995)

A basically monopole source formulation for vortex-generated sound.

Journal of the Acoustical Society of America 97(4), pp. 2144-2146.

[143] PREK, M. (2000)

Scaling laws of hydrodynamic noise generation for a simple fluid valve model.

Journal of Vibration and Acoustics, 122 (3), pp. 330-331. 
[144] PRIEDE, T. (1975)

The problems of noise of engines in different vehicle groups.

SAE Paper 750795

[145] LORD RAYLEIGH (1894)

The theory of Sound.

Second edition, London: Mcmillan.

[146] RADAVICH, P.M.; SELAMET, A. and NOVAK, J.M. (2001)

A computational approach for flow-acoustic coupling in closed side branches.

Journal of Acoustical Society of America, 109(4), pp. 1343-1353.

[147] REIBOLD, R. (1987)

Sound source reconstruction using Fourier optics.

Acustica, 63(1), pp. 60-64.

[148] REINHART, T.E. and CROCKER, M.J. (1982)

Source identification on a diesel engine using acoustic intensity measurements.

Noise Control Enginnering Journal, 18(3), pp. 84-92.

[149] RIBNER, H.S. (1996)

Effects of jet flow on jet noise via an extension to the Lighthill model.

Journal of Fluid Mechanics, 321, pp.1-24.

[150] RUBINSTEIN, R. (2000)

Formulation of a two-scale model of Turbulence.

NASA/CR-2000-209853; ICASE Report No. 2000-5. pp. 1-13.

[151] RUSSELL, M.F. and HAWORTH, R. (1985)

Combustion noise from high speed direct injection diesel engines.

SAE Paper 850973.

[152] RYHMING, I. (1895)

Dynamic des Fluides.

Press Polythecniques Romandes.

[153] SARKAR, S. and HUSSAINI, M.Y. (1993)

Computation of the sound generated by isotropic turbulence.

ICASE Report 93-94. pp. 1-18.

[154] SCHEUREN, J.; WIDMAN, U and WINKLER, J. (1999)

Active noise control and sound quality design in motor vehicles.

SAE Paper 1999-01-1846. 
[155] SEKINE,N.; MATSUMURA, S.; TAKAYAMA, K.; ONODERA, O. and ITO, K.(1988)

Shock wave development and propagation in automobile exhaust system. SAE Technical Paper 880082.

[156] SEYBERT, A.F. (1988)

Two-sensor methods for the measurement of sound intensity and acoustic properties in ducts.

Journal of Acoustical Society of America, 83(6), pp. 2233-2239.

[157] SEYBERT, A.F.; CHENG, C.Y.R. and WU, T.W. (1990)

The solution of coupled interior/exterior acoustic problems using the boundary element method.

Journal of the Acoustical Society of America, 88(3), pp. 1612-1618.

[158] SHEN, J.; MEECHAM, W.C. (1993)

Quadrupole directivity of jet noise when impinging on a large rigid plate. Journal of Acoustical Society of America, 94(3), pp. 1415-1424.

[159] SKÅNBERG, A. and ÖHRSTRÖM, E. (2002)

Adverse health effects in relation to urban residential soundscapes.

Journal of Sound and Vibration, 250(1), pp. 151-155.

[160] STAHL, V.B. and ARGÜELLO, G. (1988)

Schallerzeugung und Schalldämpfung in einer Rohrströmung stromab einer unstetigen Querschnittserweiterung.

Acustica, 65, pp. 75-84.

[161] SUCCI, GEORGE P. (1986)

Flow-Generated Noise in Circular Cavities or How Teapots Whistle.

Noise Control Engineering Journal, 26(1), pp. 8-16.

[162] TAM, C.K.W (1995)

Computational Aeroacoustics: Issues and Methods.

AIAA Journal, 33, No. 10, pp. 1788-1796

[163] TAM, C.K.W.(1998)

Jet noise: Since 1952.

Theoretical and Computational Fluid Dynamics. Springer-Verlag. Abstrat Volume 10 Issues 1-4(1998), pp. 393-405. 
[164] TAM, C.K.W. and AURIAULT, L. (1998)

Mean flow refraction effects on sound radiated from localized sources in a jet.

Journal of Fluid Mechanics, 370, pp. 149-174.

[165] THIEN, G.E. and FACHBACH, H.A. (1975)

Design concepts of diesel engines with low noise emission.

SAE Paper 750838.

[166] THOMPSON, J.K. (1982)

Acoustic intensity measurements for small engines.

Noise Control Enginnering Journal, 19(2), pp.56-63.

[167] THOMPSON, J.K.; KUNG, L.E.; TREE, D.R. (1983)

Noise source identification for three small engines.

Noise Control Enginnering Journal, 21(2), 74-80.

[168] TORREGROSA, A.J. (1993)

Método de cálculo para la predicción del ruido de admisión y escape en motores alternativos.

Tesis Doctoral. Universidad Politécnica de Valencia.

[169] TORREGROSA, A.J.; ANDRÉS, I.; MOYA, D. (2000)

Predicción del ruido de escape en presencia de flujo mediante la técnica de intensimetría en conductos.

Análes de Ingeniería Mecánica, 13(3), 2047-2052.

[170] TORREGROSA, A.J.; GALINDO, J.; BERMUDEZ, V.; ANDRÉS, I. (2003)

A study of the noise emission process in I.C. engine exhaust systems.

Journal of Sound and Vibration, en revisión.

[171] UMEDA Y. And ISHII, R. (2001)

On the sound sources of screech tones radiated from choked circular jet. Journal of Acoustical Society of America, 110(4), 1845-1858.

[172] VAN DER WALT, D.C (2001)

Measurement technique to assess the acoustic properties of a silencer component for transient engine conditions.

Journal of Sound and Vibration 243(5), pp. 797-821.

[173] VISWANATHAN, K. and SANKAR; L.N. (1995)

Toward the direct calculation of noise: Fluid/Acoustic coupled simulation.

AIAA Journal, 33, pp. 2271-2279. 
[174] WANG, M.; LELE, S.K. and MOIN, P. (1996)

Computation of quadrupole noise using acoustic analogy.

AIAA Journal, 34(11), pp. 2247-2254.

[175] WELLS, V.L. and RENAUT, R.A. (1997)

Computing aerodynamically generated noise.

Annual Review of Fluid Mechanics, 29, pp. 161-199.

[176] WADDINGTON, D.C. and OLDHAM, D.J. (1999).

Generalized flow noise prediction curves for air duct elements.

Journal of Sound and Vibration 222(1), pp. 163-169.

[177] WILLIAMS, T.J. AND COX, J.B. (1975)

The influence of exhaust poppet valve gas flow on reciprocating engine noise.

Proceceedings of the Institution of the Mechanical Engineers, 189(45), pp. 461-469.

[178] WOODRUFF, S.L.; SEINER, J.M.; HUSSAINI, M.Y. and ERLEBACHER, G. (2001)

Implementation of new turbulence spectra in the Lighthill analogy source terms.

Journal of Sound and Vibration, 242(2), pp. 197-214.

[179] YORKE, P.J. (1975)

The application of idealization and response analysis to diesel engine noise assessment.

SAE Paper 750836.

[180] ZIMMERMANN, K.D. (1975)

Injection noise and its relation to fuel pump and engine noise.

SAE Paper 750802. 